\title{
Analyse der vierperiodischen Minimalnetze
}

\author{
Dissertation \\ zur Erlangung des mathematisch-naturwissenschaftlichen Doktorgrades \\ "Doctor rerum naturalium" \\ der Georg-August-Universität Göttingen \\ im Promotionsprogramm Geowissenschaften / Geographie \\ der Georg-August University School of Science (GAUSS)
}

vorgelegt von

Alexander Beukemann

aus Stuttgart

Göttingen 2015 
Mitglieder der Prüfungskommission:

Referent/in: $\quad$ Prof. Dr. Werner Kuhs

Abteilung: Kristallographie

Fakultät für Geowissenschaften und Geographie

Georg-August-Universität Göttingen

Korreferent/in: Prof. Dr. Gerhard Scheitler

Mathematik, Wirtschaft und Technik

Fachhochschule für Oekonomie und Management

FOM München

2. Korreferent/in: apl. Prof. Dr. Heidrun Sowa

Abteilung: Kristallographie

Fakultät für Geowissenschaften und Geographie

Georg-August-Universität Göttingen

Weitere Mitglieder der Prüfungskommission:

Prof. Dr. Sharon Webb

Abteilung: angewandte und experimentelle Mineralogie

Fakultät für Geowissenschaften und Geographie

Georg-August-Universität Göttingen

PD Dr. Helmut Klein

Abteilung: Kristallographie

Fakultät für Geowissenschaften und Geographie

Georg-August-Universität Göttingen

Dr. Patrick Lafond

Abteilung: Kristallographie

Fakultät für Geowissenschaften und Geographie

Georg-August-Universität Göttingen

Tag der mündlichen Prüfung: 11.02.2015 


\section{Danksagung}

Mein ausdrücklicher Dank gilt Herrn Prof. Dr. Werner Kuhs, der es mir ermöglicht hat, diese Doktorarbeit zu schreiben, Herrn Prof. Dr. Gerhard Scheitler für die Übernahme des Korreferats, und Frau apl. Prof. Dr. Heidrun Sowa, die mich bei der Literaturrecherche sehr unterstützt hat und das 2. Korreferat übernommen hat.

Mein Dank gilt ferner

Jürgen Saeftel für die Aufnahme des Kristallmodells, die professionelle Bearbeitung des Photos, und für die Durchsicht der Arbeit.

Fritz Krien für die Durchsicht der Arbeit.

Christine, Lisa und Finja - meinem Dreimaderlhaus - dafür, dass sie mich bei diesem Vorhaben über die gesamte Zeit seelisch unterstützt, großes Interesse an der Arbeit gezeigt und mich kulinarisch verwöhnt haben. 


\begin{abstract}
Periodic structures - e. g. crystal structures - are analyzed by means of group theory. The concept of a crystal structure is extendable to the n-dimensional space and the 4-dimensional space groups are known [Brown, Bülow, Neubüser, Wondratscheck, Zassenhaus, 1978]. As quasi-periodic and incommensurate structures can be the result of a projection of a higher periodic structure, it is also worth to enhance the knowledge about higher-periodic structures by graph theoretical means. Thereby the atoms of a crystal can be assigned to the vertices, and the chemical bonds to the edges of a graph being a periodic net. Minimal nets [Beukemann, Klee, 1992] are nets, where the deletion of any edge together with and its translational equivalent edges results in a structure that is no longer connected. It's special about a minimal net to have a 1:1 relation to its quotient graph [Chung, Hahn, Klee, 1984] that represents the topology of the net by a finite graph, presenting the adjacency relations between the classes of translational equivalent vertices and edges of the periodic net. 3-periodic minimal nets are analyzed by several authors such as Bonneau, Delgado-Friedrichs, O'Keeffe, Yaghi, Eon. Now the complete class of 4-periodic minimal nets (111 nets) is analyzed by comparing their substructures such as trees, chains, cycles and sub-networks. Net to subnet relations are identified and methods for net conversions are proposed. Different principles for the construction of those structures are applied. The embedding of two nets is discussed in detail. Single cycles of the minimal net 6(3)1 - number 1 of minimal nets with 6 classes of vertices and vertex degree 3 (quotient graph $\mathrm{K}_{3,3}$ ) - where mapped to polygons of a Penrose pattern. The minimal net 3(4)1 could be embedded into the 4-dimensional space by a "symmetric labelling" of the edges of its quotient graph. A reference work with graphics and cycle class sequences of all 2-, 3- and 4-periodic minimal nets is attached.
\end{abstract}




\section{Inhaltsverzeichnis}

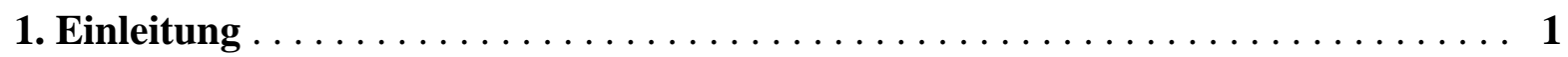

2. Grundlagen $\ldots \ldots \ldots \ldots \ldots \ldots \ldots \ldots \ldots \ldots \ldots \ldots \ldots \ldots \ldots \ldots \ldots, 8$

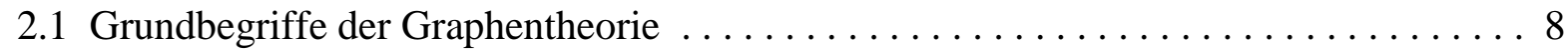

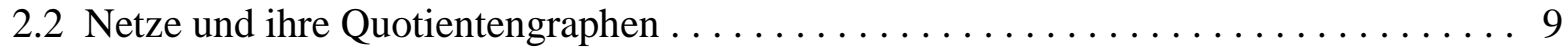

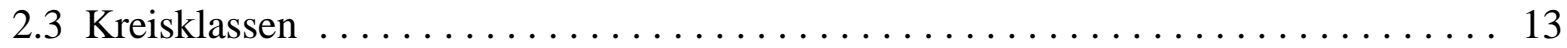

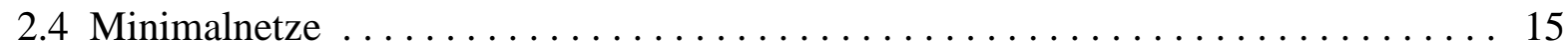

3. Substrukturen vierperiodischer Minimalnetze $\ldots \ldots \ldots \ldots \ldots \ldots \ldots \ldots$

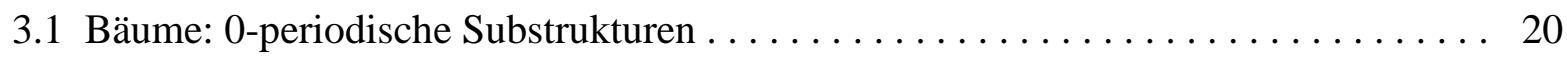

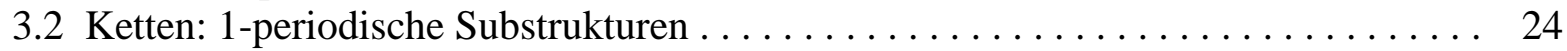

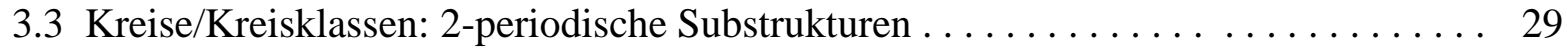

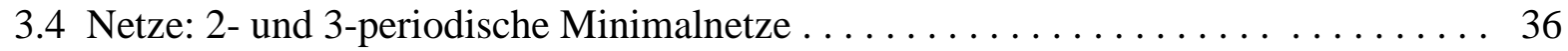

4. Erzeugen von 4p-Minimalnetzen aus 4p-Minimalnetzen

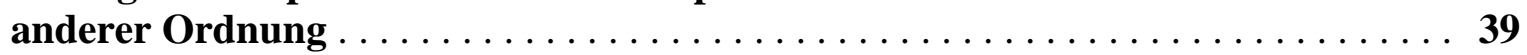

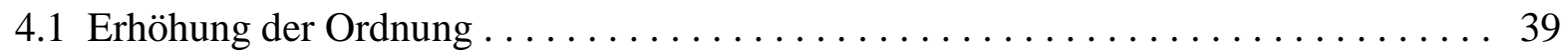

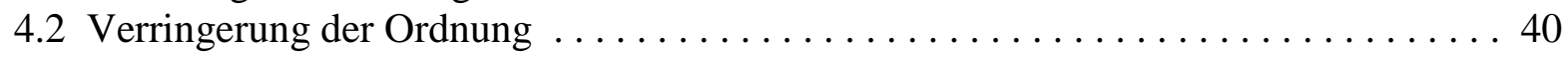

5. Erzeugung von 4p-Minimalnetzen aus 3p-Netzen mit gleichem Quotientengraphen $\ldots \ldots \ldots \ldots \ldots \ldots \ldots \ldots \ldots \ldots \ldots \ldots$

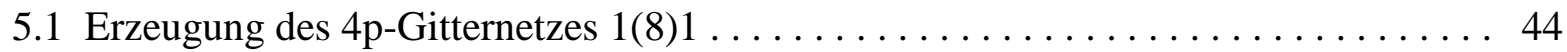

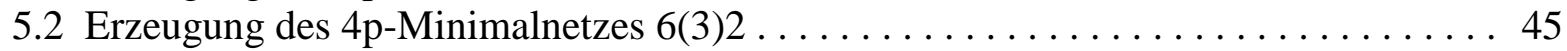

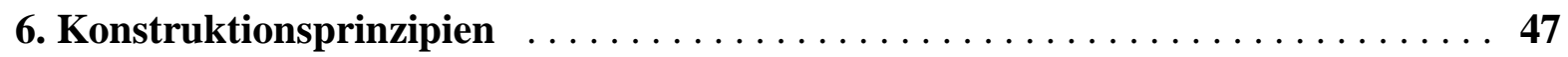

6.1 Darstellung des Minimalnetzes $5\left(3^{4}, 4\right) 4 \ldots \ldots \ldots \ldots \ldots \ldots \ldots \ldots \ldots \ldots$

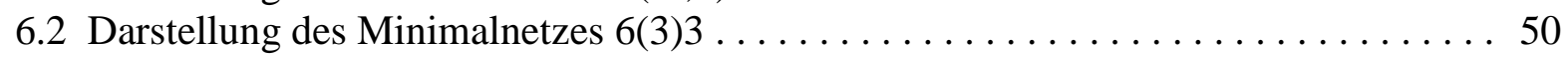

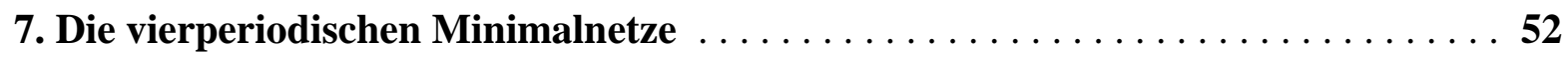

7.1 Das 4-periodische Gitter - Minimalnetz $1(8) 1 \ldots \ldots \ldots \ldots \ldots \ldots \ldots \ldots \ldots \ldots \ldots \ldots$

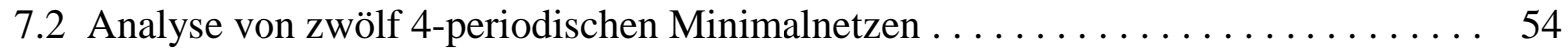

8. Minimalnetz 6(3)1 - Penrose-Muster zur Konstruktion der 12er-Kreise $\ldots \ldots \ldots 61$

9. Einbettung des Minimalnetzes 3(4)1 mit symmetrischer Indizierung $\ldots \ldots \ldots 68$

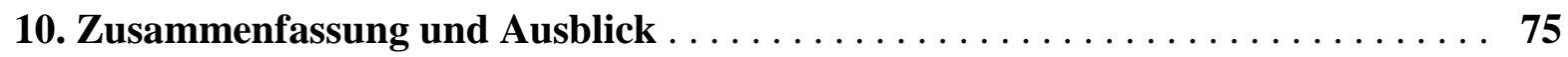

Anhang: Die 3 zwei- , 15 drei- und 111 vierperiodischen Minimalnetze $\ldots \ldots \ldots 79$

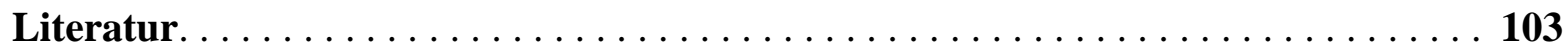




\section{Einleitung}

Das besondere Merkmal einer Kristallstruktur ist seine Periodizität. Daraus folgt, dass die Kenntnis eines kleinen räumlichen Bereichs (einer Elementarzelle) Aufschluss über die gesamte Kristallstruktur gibt.

Da es Beziehungen zwischen physikalischen Eigenschaften eines Kristalls und seiner Struktur gibt, und um die Kristallstrukturen zu klassifizieren, werden ihre geometrischen Eigenschaften (gruppentheoretisch) oder ihre Netzstrukturen (graphentheoretisch) analysiert und miteinander verglichen.

Die gruppentheoretische Betrachtung von Kristallstrukturen basiert auf dimensionalen Größen wie Längen und Winkel, und auf den Symmetrieoperationen, die diese Struktur mit sich zur Deckung bringen. Symmetrieoperationen sind Translationen, Drehungen (2-,3-, 4- und 6-zählig), Schraubungen, Spiegelungen, Drehspiegelungen und die Identität. Die Gesamtheit aller möglichen Symmetrieoperationen einer kristallographischen Struktur bildet eine Raumgruppe.

In Folge des Gitteraufbaus hängen in Kristallen physikalische Größen, denen eine Richtung zugewiesen werden kann - wie z. B. Festigkeit, Leitfähigkeit (Strom, Wärme), optisches Verhalten -, von der Symmetrie des Kristalls und von der Richtung der physikalischen Größe ab (Anisotropie). [Kleber, Bautsch, Bohm, Klimm, 2010; Klockmann, 1980].

Das Konzept einer Kristallstruktur kann auf den n-dimensionalen Raum erweitert werden und ist eine n-fach-periodische Anordnung von Punkten; unter den Abbildungen gibt es $\mathrm{n}$ Translationen mit linear unabhängigen Translationsvektoren einer bestimmbaren Mindestlänge $(\mathrm{d}>0)$, die das Objekt auf sich selbst abbilden. In vierdimensionalen kristallographischen Gruppen können auch 5-, 8- 10- und 12-zählige Symmetrieoperationen vorkommen; ihre kompletten Tabellen wurden erstmals in [Brown, Bülow, Neubüser, Wondratscheck, Zassenhaus, 1978] präsentiert.

Im April 1982 entdeckte Daniel Shechtman auf Strukturaufnahmen einer Legierung aus Aluminium und Mangan mit Elektronenstrahlen Muster mit 10-zähliger Drehsymmetrie. In dieser Struktur lässt sich kein Motiv finden, welches sich periodisch wiederholt, jedoch kann über spezielle Anbauregeln eine unendliche (quasiperiodische) Struktur erzeugt werden [Shechtman, 2013]. Für die Entdeckung der „Quasikristalle“ wurde er 2011 mit dem ChemieNobelpreis ausgezeichnet. Inzwischen konnte in zahlreichen, vor allem intermetallischen Systemen, die Existenz quasikristalliner Strukturen nachgewiesen werden [Steurer, 2004]. Eine wichtige Methode, solche Strukturen zu beschreiben und zu analysieren ist die höherdimensionale Kristallographie, die von de Wolff zur Beschreibung inkommensurabel modulierter Strukturen [De Wolff, 1984] entwickelt wurde.

Eine andere Möglichkeit, Kristallstrukturen zu beschreiben, ist durch die Graphentheorie und durch das Konzept der Kugelpackungen gegeben. Die Struktur wird dabei als System miteinander vernetzter Atome betrachtet.

Beim Konzept der Kugelpackungen werden Atome oder Ionen als starre Kugeln angesehen. In der Kristallographie wird eine Ansammlung von sich nicht überschneidenden Kugeln mit der Symmetrie einer kristallographischen Gruppe dann Kugelpackung genannt, wenn zwei beliebige Kugeln daraus über eine Kette sich berührender Kugeln verbunden sind [Fischer, 1991]. In der Natur kommen unterschiedliche Bauprinzipien vor, z. B. dichteste Packung, höchste Symmetrie, höchstmöglichste Koordination. 
Bei der graphenheoretischen Betrachtung einer Kristallstruktur werden die Atome als Punkte oder Knoten, die chemischen Bindungen zwischen den Atomen als Linien oder Kanten (Abb. 1.1), und die aus ihnen bestehende Struktur als Graph bezeichnet.

Die Knoten entsprechen bei Kugelpackungen den Kugelmittelpunkten, und die Knoten zweier sich berührender Kugeln sind durch eine Kante verbunden. Jede Kugelpackung kann eindeutig einem Graphen zugeordnet werden [Fischer, 1991].

Graphen von Kristallstrukturen werden, wenn sie dreifach zusammenhängend sind (Kap. 2.2), aufgrund der periodischen Anordnung ihrer Knoten und Kanten als periodische Netze bezeichnet.
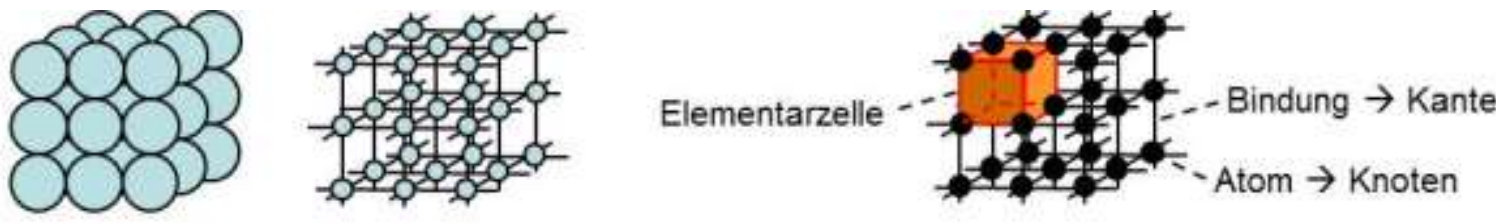

Abb. 1.1 Kugelpackung und periodisches Netz. Die Mittelpunkte der Kugeln entsprechen den Knoten eines Graphen, die im Graphen durch eine Kante verbunden werden, wenn sie sich berühren. Links die Darstellung des periodischen Netzes mit einer Elementarzelle (durch sie wird der gesamte Aufbau des Kristalls beschrieben).

Die wesentliche treibende Kraft für Atome, untereinander in Wechselwirkung zu treten und sogenannte chemische Bindungen auszubilden, ist das Erreichen stabiler Elektronenkonfigurationen. Dabei gibt es verschiedene Bindungsszenarien [Kickelbick, 2008; Bannwarth, Kremer, Schulz, 2010]:

- Kovalente Bindung: gemeinsame Elektronenpaare der Bindungspartner (Abb. 1.2)

- Ionenbindung: Elektronenübergang von einem auf den anderen Partner (Abb. 1.2)

- Metallische Bindung: Abgabe von Valenzelektronen an die Elektronenwolke, die die Atome umgibt. (Abb. 1.2)

- Zwischenmolekulare Wechselwirkungen:. Wasserstoffbrückenbindungen (Abb. 1.3), Van-der-Waals- und Dipolwechselwirkungen.

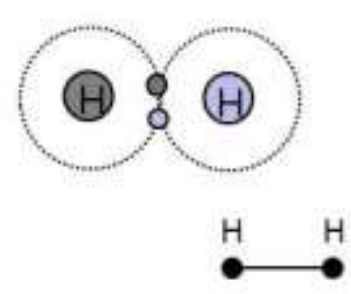

Kovalente Bindung: $\mathrm{H}_{2}$
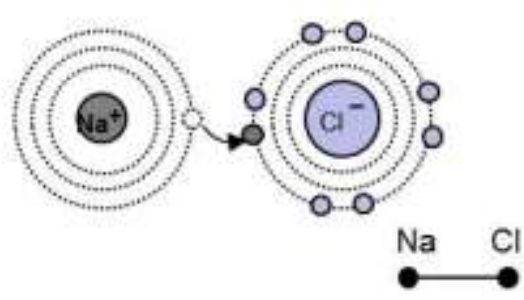

lonenbindung: $\mathrm{NaCl}$

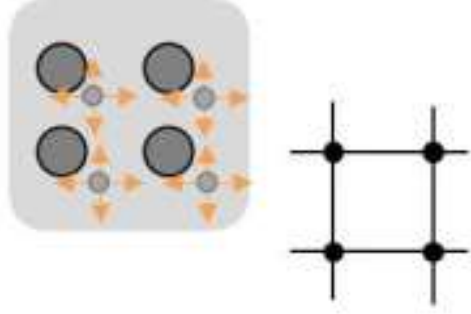

Metallische Bindung: Metalle

Abb. 1.2 Darstellung von Atomen und ihren Bindungen als Graph: Kovalente Bindung und Ionenbindung (gepunktete Linien: Elektronenschalen), Metallbindung (nach dem Elektronengasmodell sind die Valenzelektronen keinem einzelnen Atom zuzuordnen [Kickelbick, 2008]); jeweils linksunten daneben die Darstellung als Graph. 
Das Konzept periodischer Netze [Wells, 1977] spielt mittlerweile eine bedeutende Rolle in der modernen Kristallchemie, um topologische Eigenschaften von Kristallstrukturen zu analysieren [Blatov, 2007].

Ein vollständiges Netz enthält alle Atome als Knoten und alle interatomaren Beziehungen als Kanten [Blatov, Proserpio, 2011].

Partielle Netze entstehen durch Vereinfachung der Netzstruktur, z. B. durch das

- Weglassen bestimmter Kanten,

- Weglassen bestimmter Knoten zusammen mit ihren Verbindungskanten,

- Zusammenziehen mehrerer verbundener Knoten (Cluster) zu einem Knoten.

[Blatov, Proserpio, 2011; Delgado-Friedrichs, O’Keeffe, 2003]

Bei bestimmten kristallchemischen Untersuchungen werden partielle Netze betrachtet, indem nur Verbindungen mit Bindungskräften gewisser Stärke berücksichtigt werden, oder die Zwischenräume-bildenden Ionen oder Moleküle in gewissen Gerüststrukturen weggelassen werden [Blatov, Proserpio, 2011]. So werden in metallorganischen Kristallen (auch MOF = metall organic frameworks) molekulare Strukturen über Wasserstoff(brücken)bindungen verbunden und bilden eine supramolekulare Architektur. Diese kann als 3-dimensionales Netz aufgefasst werden (Abb. 1.3) [Baburin, 2008].
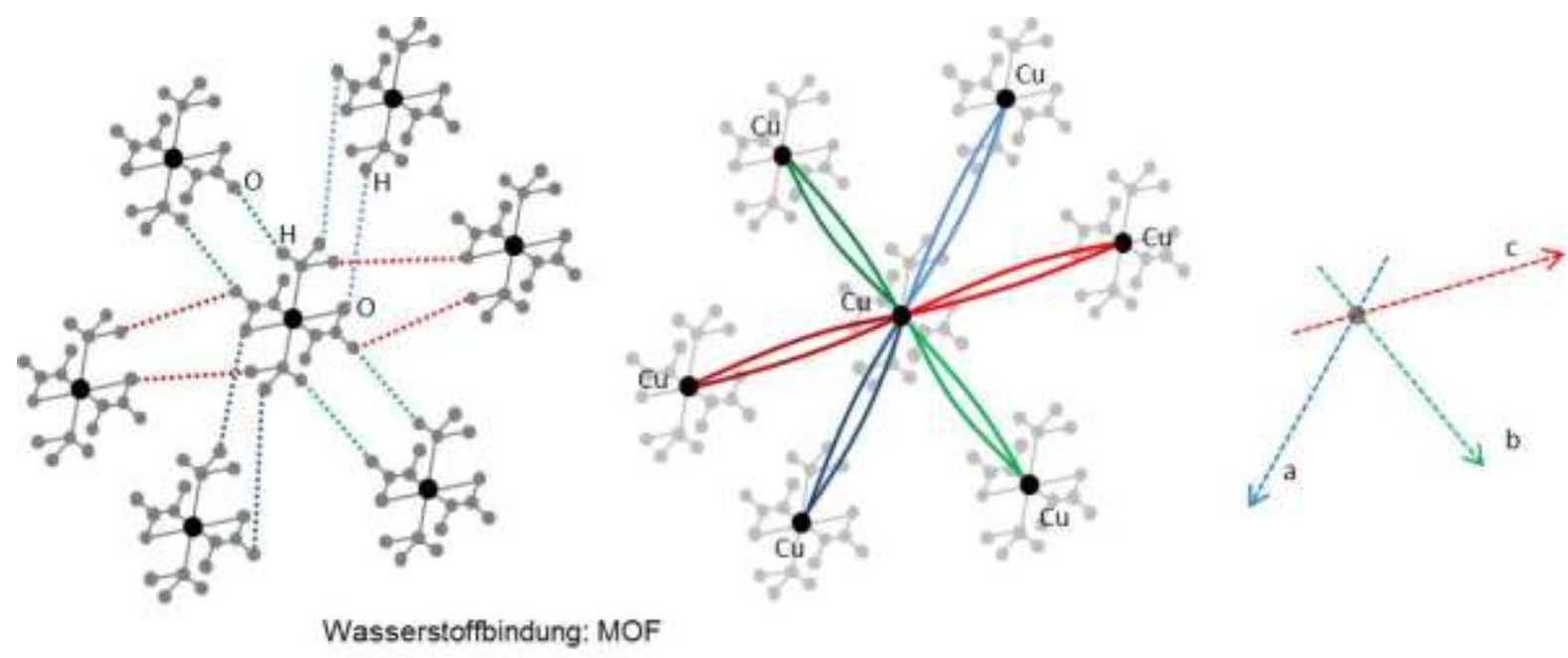

Abb.1.3 Wasserstoffbindungen in metallorganischen Kristallen. Vereinfachte Darstellung von Fig. 1 in [Baburin, 2008]. Die Cu-Atome (schwarz) sind die Knoten, und die Wasserstoffbindungen (farbig) sind die Doppelkanten eines 3-dimensionalen Netzes (rechts).

Da es unendliche viele periodische Netze gibt (denn es gibt keine Begrenzung für die Anzahl der Knoten- und Kantenklassen), können nur Untermengen dieser Netze analysiert werden. Spezielle Netze sind z. B.:

- Uninodale Netze: Netze mit einer Knotenart

- Kantentransitive Netze: Netze mit einer Kantenart

- Minimalnetze: Netze, die zerfallen, wenn eine Kante und die zu ihr translatorisch gleichwertigen Kanten gelöscht werden. 
Die Topologie periodischer Netze, die durch seine Knoten und deren Nachbarschaftsverhältnisse untereinander gegeben ist, lässt sich durch Graphen unterschiedlicher Art darstellen [Klein, 2012].

Quotientengraphen [Chung, Hahn, Klee, 1984] sind Graphen, in denen jeder Knoten eine Klasse translatorisch gleichwertiger Knoten, und jede Kante eine Klasse translatorisch gleichwertiger Kanten repräsentiert.

Eine Methode, um ein periodisches Netz durch einen Quotientengraphen darzustellen, besteht darin, eine primitive Elementarzelle so zu wählen, dass in ihr von jeder Knotenklasse genau ein Knoten enthalten ist, und diese Knoten innerhalb der Zelle einen zusammenhängenden Graphen bilden. Kanten, die von dieser Zelle in benachbarte Zellen führen, werden entsprechend der relativen Position dieser Zellen zur Ausgangszelle indiziert.

Zur Beschreibung von Minimalnetzen eignen sich Quotientengraphen, deren Elementeanzahl der Anzahl der Elemente einer primitiven Elementarzelle entspricht, besonders gut, weil es eine 1:1-Beziehung zwischen dem Netz und seinem Quotientengraphen gibt, und eine lückenlose Erzeugung aller Minimalnetze einer gewissen Periodizität über Quotientengraphen möglich ist. (Werden nicht-primitive Elementarzellen zur Erzeugung des Quotientengraphen gewählt, so resultieren daraus Quotientengraphen höherer Ordnung [Sunada, 2013, Fig 3.7]).

Es gibt genau drei Minimalnetze mit einer zweifachen Periodizität (Abb. 1.4). Zwei von ihnen lassen sich in einen zweidimensionalen Raum einbetten, das dritte dagegen nicht.

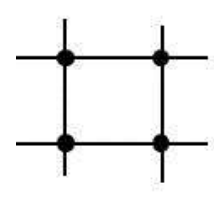

a

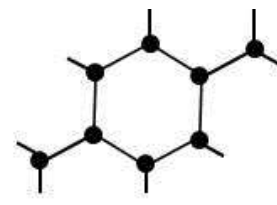

b

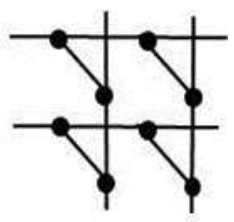

c

Abb. 1.4 Einbettung von 2-periodischen Minimalnetzen. Die beiden Netze (a) und (b) lassen sich in die Ebene einbetten, das Netz (c) nicht, denn es kreuzen sich Kanten auch an Stellen, an denen sie nicht über einen Knoten verbunden sind.

„Eine Einbettung eines Graphen ist eine Darstellung desselben in einen Raum einer geeigneten Dimension, in welcher die Knoten und Kanten so durch Punkte und Linien dargestellt sind, dass die Inzidenzrelationen durch Linien, die sich nicht kreuzen, dargestellt werden“" [Thimm, 1991].

Werden Kristallstrukturen durch Netze beschrieben, können chemische und geometrische Eigenschaften die Einbettungsbedingung einschränken. So spricht man von einer geeigneten Einbettung einer Kristallstruktur in den Raum, wenn der Abstand zwischen Punkten, die nicht miteinander verbunden sind, größer als der Abstand miteinander verbundener Punkte ist [Bonneau, Delgado-Friedrichs, O'Keeffe, Yaghi, 2004; Eon 2007 und 2011; Thimm, 2008].

Die fünfzehn dreifachperiodischen (= dreiperiodischen) Minimalnetze sind als 3-dimensionale Strukturen perspektivisch dargestellt worden [Beukemann, Klee, 1992]. Acht von ihnen erfüllen die Kriterien für die geeignete Einbettung einer Kristallstruktur [Bonneau, DelgadoFriedrichs, O’Keeffe, Yaghi, 2004].

Um vier- oder höherperiodische Strukturen auf „geeignete Weise“ einbetten zu können, wird ein Raum entsprechender Dimension (> 3) benötigt. 
Die Theorie höherer Dimensionen wurde am 10.6.1854 durch Bernhard Riemann in einer Probevorlesung an der Universität Göttingen eingeführt.

Mithilfe von Analogien brachten Mathematiker wie z. B. Gauß diese neuen mathematischen Erkenntnisse der Vorstellungskraft näher [Behrends, Gritzmann, Ziegler, 2008]. So lässt sich der Satz von Pythagoras auf $\mathrm{n}$ Dimensionen verallgemeinern und die Diagonale eines Hyperwürfels (Abb. 1.5) berechnen:

$\mathrm{z}^{2}=\mathrm{a}^{2}+\mathrm{b}^{2}+\mathrm{c}^{2}+\mathrm{d}^{2}$

mit $a, b, c, d, \ldots$ als Kantenlängen und $z$ als die Länge der Diagonalen.

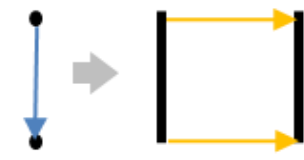

Strecke

Quadrat

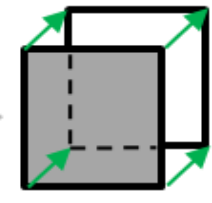

Würfel

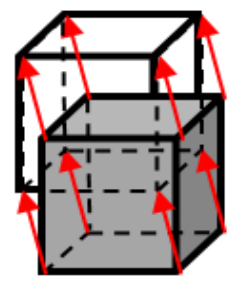

Hyperwürfel

\section{Abb. 1.5 Hyperwürfel}

In Einsteins Konzept ist die 4. Dimension die Zeit. In der mehrdimensionalen Kristallographie werden räumliche (voneinander linear unabhängige) Dimensionen betrachtet, und somit ist die 4. Dimension eine weitere räumliche Dimension.

Quasikristalle haben eine aperiodische Struktur und können deshalb im 3-dimensionalen Raum nicht als periodisches Netz beschrieben werden, jedoch können quasiperiodische Strukturen durch die Projektion von periodischen Strukturen einer höheren (> 3) Dimensionalität in den 3-dimensionalen Raum entstehen. [Meisterernst, 2006]. Abb. 1.6 zeigt die Projektion einer 2-dimensionalen periodischen Struktur in einen 1-dimensionalen Raum.

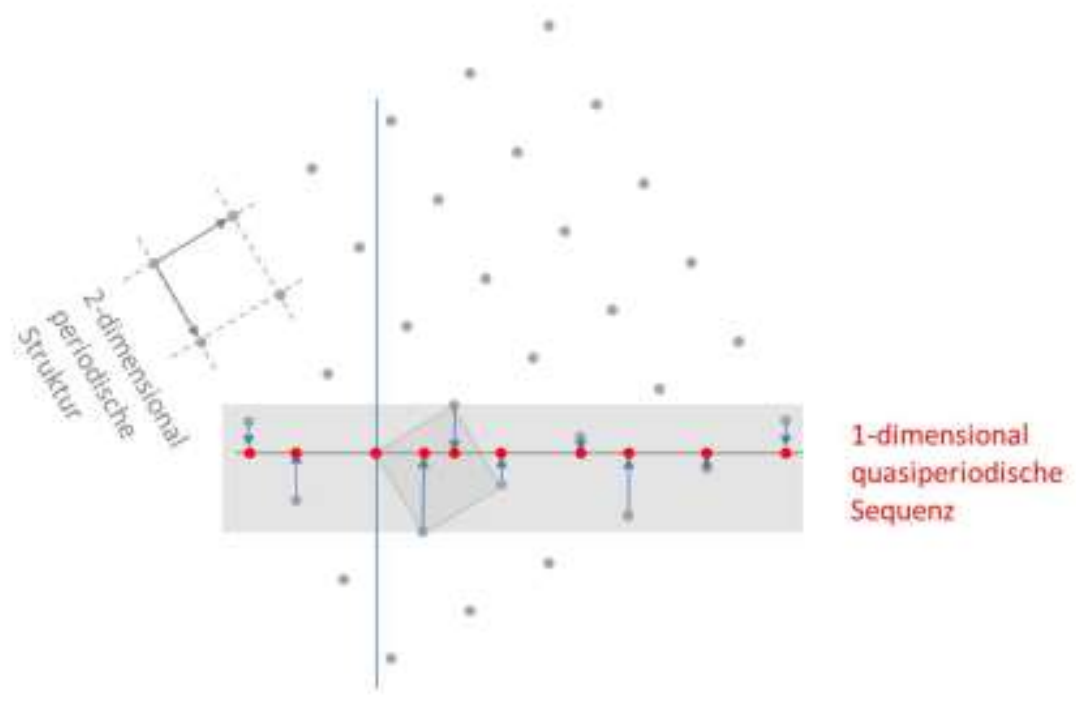

Abb. 1.6 Quasiperiodische Sequenz. Diese Abbildung entspricht inhaltlich der Abb. 2.3 in [Meisterernst, 2006] mit der Bildunterschrift: „Die Projektion einer zweidimensional periodischen Struktur durch einen irrationalen Schnitt erzeugt eine 1d-quasiperiodische Sequenz“. 
Der einfachste Fall des quasiperiodischen Zustandes ist ein inkommensurabel modulierter (durch eine periodische Störung verzerrter), periodischer Kristall: Rubidiumtetrachlorozinkat $\mathrm{Rb}_{2} \mathrm{ZnCl}_{4}$ ist ein Beispiel für einen Festkörper, bei dem bei Temperaturänderung ein Phasenübergang zwischen kommensurabler und inkommensurabler Phase stattfindet [Elisbihani, 2002].

Neuere Entwicklungen in der Theorie der Quasikristalle und inkommensurablen Strukturen führen nun zu erhöhtem Interesse an der Kristallographie mit mehr als drei Dimensionen. Für manche Strukturtypen, wie z. B. für Diamant, wurde eine Verallgemeinerung auf n Dimensionen vorgenommen [O’Keeffe, 1991; Eon, 2004].

In der vorliegenden Arbeit werden nun bestimmte Netze untersucht, die aufgrund ihrer Periodizität den 4-dimensionalen Raum in Anspruch nehmen, und zwar die:

$\rightarrow 111$ vierfachperiodischen (= vierperiodischen) Minimalnetze

Die Punkte eines 4-dimensionalen Gitters sind in vier voneinander linear unabhängigen Richtungen periodisch angeordnet. Werden sie entlang dieser Richtungen miteinander verbunden, so entsteht ein 4-periodisches (Gitter-)Netz.

Werden größere Ausschnitte einer Konstruktion eines vierperiodischen Minimalnetzes auf die Ebene projiziert, dann entstehen schwierig interpretierbare Netze (Abb. 1.7 links). Um die Darstellungen interpretieren zu können, werden sie auf kleine Ausschnitte beschränkt, wobei die Netzstruktur vollständig repräsentiert wird (Abb. 1.7 rechts unten).

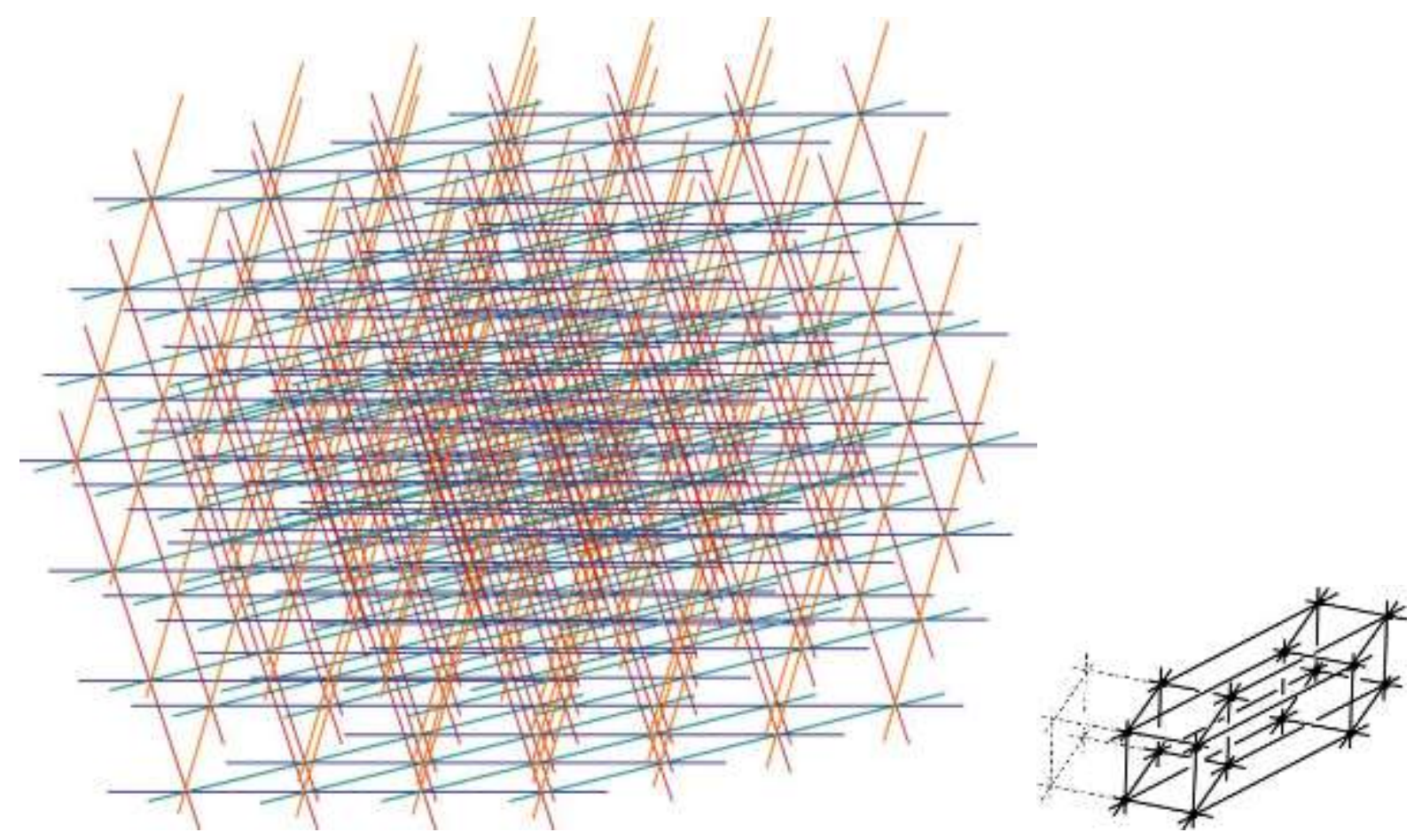

Abb. 1.7 Vierdimensionales Gitternetz, auf die Ebene projiziert. Ein kleiner Ausschnitt (rechts) eines periodischen Netzes kann die Netzstruktur vollständig repräsentieren. 
Um Darstellungen für die Netze zu finden, die die Hauptmerkmale ihrer Struktur in angemessener Weise zeigen, und die die Unterschiede zwischen den einzelnen Netzen deutlich machen, werden ihre Substrukturen eingehend untersucht.

Die Analyse der Substrukturen kann als Entscheidungshilfe für die Wahl einer bestimmten Konstruktion dienen. In periodischen Netzen bieten sich folgende Substrukturen an:

- Bäume

- Ketten

- Kreise

- Netze

Kreise, die translatorisch gleichwertig sind, gehören einer Kreisklasse an. Da sich die 111 Netze alle in Bezug auf ihr Kreisklassenspektrum unterscheiden, kommt dieser Sequenz eine besondere Bedeutung zu:

- Die Betrachtung der Kreisklassen eines Netzes ermöglicht eine Analyse auf anschauliche und eindrucksvolle Weise.

- Die einzelnen Netze lassen sich in Scharen 2-periodischer Substrukturen, die aus Kreisen einer Kreisklasse gebildet werden, zerlegen.

Auch Gemeinsamkeiten und Ähnlichkeiten unterschiedlicher Netze werden betrachtet.

- Netze können gleiche Substrukturen haben

- Netze können durch definierte Umwandlungsprozesse auseinander hervorgehen.

Zwei 4-periodische Minimalnetze werden auf besondere Weise in den 4-dimensionalen Raum eingebettet:

- Bei einem Netz werden kleine Teilstrukturen (Kreise der Länge 12) mit Polygonen eines Penrose-Musters [Penrose, 1979] zur Deckung gebracht.

- Das andere Netz wird symmetrisch eingebettet, und die Koordinaten seiner Knoten werden bestimmt.

Zusammenfassend gibt diese Arbeit einen umfassenden Einblick in die komplette Klasse der 4-periodischen Minimalnetze und betrachtet ihre graphentheoretischen Aspekte. Eine kompakte Übersicht dieser Netze zusammen mit ihren Quotientengraphen und ihren Kreisklassenspektren befindet sich im Anhang. 


\section{Grundlagen}

\subsection{Grundbegriffe der Graphentheorie}

Für Definitionen aus dem Bereich der Graphentheorie verweise ich auf [Harary, 1969], jedoch werden in der folgenden Arbeit die Elemente eines Graphen grundsätzlich als Knoten und Kanten bezeichnet.

Ein Graph besteht aus einer nicht leeren Menge von Knoten und aus Paaren dieser Elemente. Die Verbindungen zwischen den beiden Knoten eines Paares werden Kanten genannt.

Ist ein Knoten über eine Kante mit sich selbst verbunden, so wird diese Kante Schleife genannt. Sind zwei Knoten über mehrere Kanten direkt miteinander verbunden, so liegt eine Mehrfachkante vor. Ein Graph heißt schlicht, wenn er weder Schleifen noch Mehrfachkanten besitzt. Bei einem vollständigen Graphen $\mathrm{K}_{\mathrm{n}}$ sind alle $\mathrm{n}$ Knoten paarweise miteinander verbunden (Abb. 2.1).

Eine Kantenfolge ist eine alternierende Folge von Knoten und Kanten, die mit je einem Knoten beginnt und endet, und in welcher jede Kante mit denjenigen beiden verschiedenen Knoten inzidiert, die in der Folge unmittelbar neben ihr stehen. Ein Kreis ist eine geschlossene Kantenfolge mit $\mathrm{n} \geq 3$ Knoten, bei der alle Knoten und Kanten verschieden sind. Die Länge eines Kreises ist durch die Anzahl seiner Kanten gegeben. Ein Baum ist ein kreisloser Graph (Abb. 2.1).
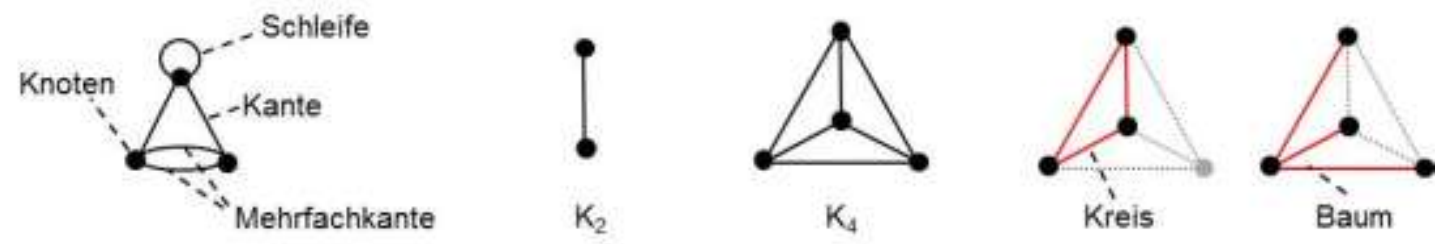

Abb. 2.1 Begriffe aus der Graphentheorie. $K_{2}$ und $K_{4}$ sind vollständige Graphen. Zu Kreis und Baum (die beiden Skizzen rechts) gehören jeweils die roten Kanten und die schwarzen Knoten.

Ein paarer Graph ist ein Graph, dessen Knotenmenge sich in zwei Teilmengen zerlegen lässt, sodass jede Kante einen Knoten der einen Menge mit einem der anderen Menge verbindet. Beim vollständig paaren Graphen $\mathrm{K}_{3,3}$ sind alle drei Knoten der einen Menge mit allen drei Knoten der anderen Menge über Kanten verbunden. Graphen können aus anderen Graphen zusammengesetzt sein. Der Graph $\mathrm{K}_{2} \mathrm{x} \mathrm{K}_{3}$ kann aus den Graphen $\mathrm{K}_{2}$ und $\mathrm{K}_{3}$ gebildet werden (Abb. 2.2).

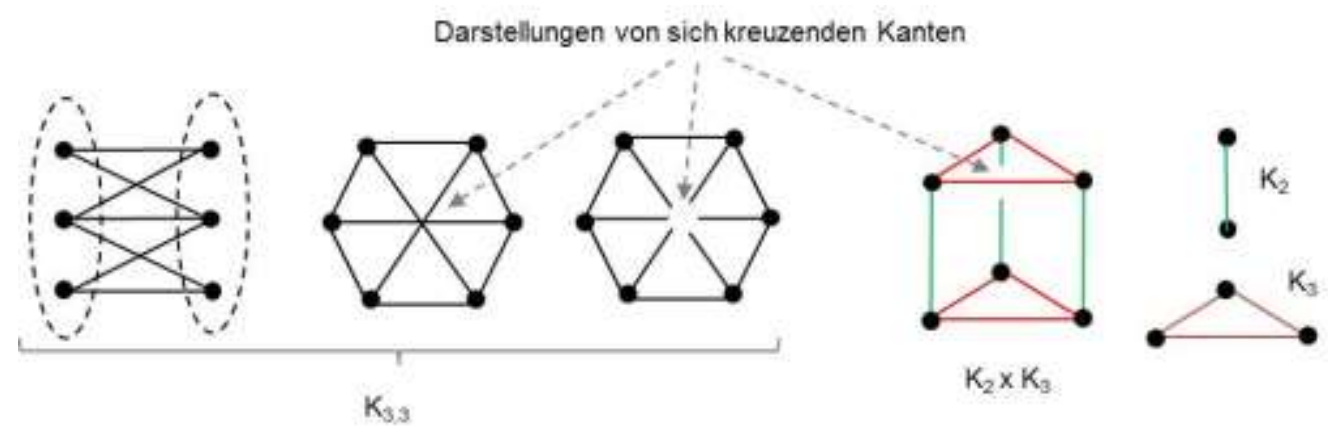

Abb. 2.2 Die Graphen $\mathbf{K}_{3,3}$ und $\mathbf{K}_{\mathbf{2}} \mathbf{x} \mathbf{K}_{3}$. Die drei Abbildungen des $K_{3,3}$ sind topologisch gleichwertig. Wenn sich Kanten in einer 2-dimensionalen Abbildung überkreuzen, jedoch nicht miteinander verbunden sind, werden sie manchmal „unterbrochen“ (3. und 4. Skizze von links) dargestellt. 


\subsection{Netze und ihre Quotientengraphen}

Der Begriff Netz wird in dieser Arbeit für schlichte Graphen mit dreifachem Zusammenhang benutzt: das heißt der Graph ist, wenn zwei beliebig ausgewählte Kanten gelöscht werden, noch zusammenhängend. Der Knotengrad eines jeden Knotens muss mindestens 3 betragen; jeder Knoten ist somit über mindestens drei Kanten mit anderen Knoten verbunden. Knoten mit Knotengrad 1 und 2 kommen in Netzen hier somit nicht vor.

Die Wiederholung eines Ereignisses oder einer Struktur in regelmäßigen zeitlichen oder räumlichen Abständen wird Periode genannt. Ein Netz ist n-periodisch, wenn es n-fache Translationssymmetrie besitzt, $\mathrm{d}$. $\mathrm{h}$. wenn es durch Translationsvektoren in $\mathrm{n}$ voneinander unabhängige Richtungen auf sich selber abgebildet werden kann. Jedes n-periodische Netz kann durch eine primitive Elementarzelle - einen kleinstmöglichen Bereich - beschrieben werden, die durch $\mathrm{n}$ linear unabhängige Basisvektoren aufgespannt wird.

Translatorisch gleichwertig werden zwei Knoten bzw. Kanten genannt, wenn sie durch eine Linearkombination der Basisvektoren miteinander zur Deckung gebracht werden können. Translatorisch gleichwertige Knoten gehören einer Knotenklasse, translatorisch gleichwertige Kanten einer Kantenklasse an (Abb. 2.3).

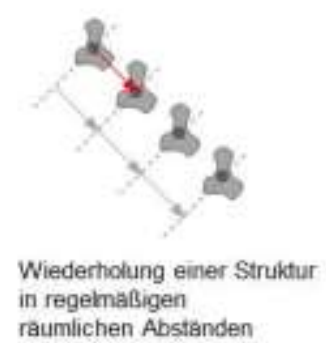

raumlichen Abstanden
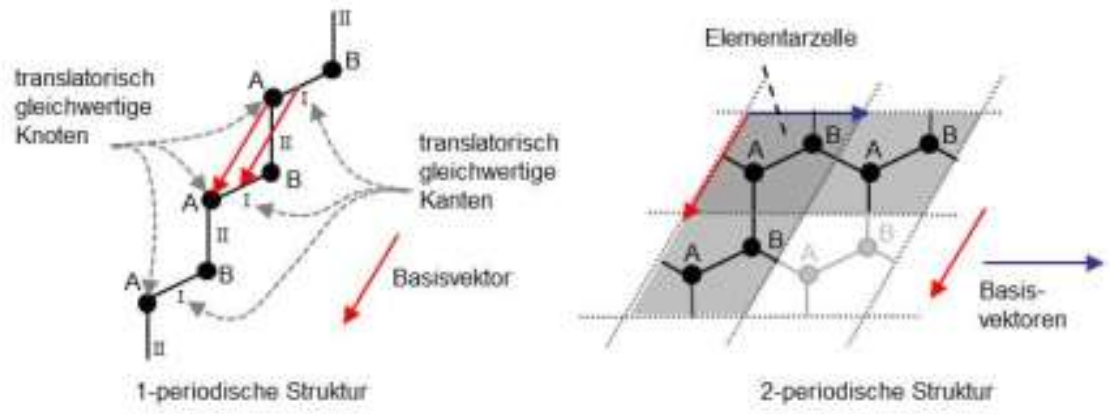

Abb. 2.3 Periodizität, translatorisch gleichwertige Knoten und Kanten, Basisvektoren.

Mitte: 1-periodische Struktur (Kette). Rechts: 2-periodisches Netz. Vektoren, die durch Verschiebung der Struktur diese mit sich selbst zur Deckung bringen, lassen sich durch Linearkombinationen der Basisvektoren bilden (Translationsvektoren).

Wird ein periodisches Netz so in den Raum eingebettet, dass es maximale Translationssymmetrie besitzt (d. h. die Einbettung der Knoten wird durch eine minimale Anzahl von Punktgittern realisiert), kann ein endlicher Graph auf folgende Weise zugeordnet werden [Chung, Hahn, Klee, 1984]:

- Die Punktgitter $\mathbf{P}_{\mathbf{1}}, \mathbf{P}_{2}, \ldots . \mathbf{P}_{\mathbf{P}}$ (ein Punktgitter entspricht einer Klasse translatorisch gleichwertiger Knoten) werden den Knoten $P_{1}, P_{2}, \ldots P_{P}$ (Repräsentanten der unterschiedlichen Knotenklassen) im endlichen Graphen zugeordnet.

- Die Liniengitter (ein Liniengitter entspricht einer Klasse translatorisch gleichwertiger Kanten) werden den Kanten (Repräsentanten dieser Kantenklassen) so im endlichen Graphen zugeordnet, dass ein Liniengitter, welches die Punkte von $\mathbf{P}_{\mathbf{i}}$ mit den Punkten von $\mathbf{P}_{\mathbf{j}}$ verbindet, einer Kante im endlichen Graphen entspricht, die dort $P_{i}$, und $P_{j}$, verbindet.

- Verbindet ein Liniengitter die Punkte eines Punktgitters $\mathbf{P}_{\mathbf{i}}$, so werden im endlichen Graphen seine Kanten einer Schleife an Punkt $P_{i}$, zugeordnet.

- Verbinden mehrere Liniengitter die Punkte von $\mathbf{P}_{\mathbf{i}}$ mit den Punkten von $\mathbf{P}_{\mathbf{j}}$, so entsprechen die einzelnen Liniengitter den einzelnen Kanten einer Mehrfachkante zwischen $P_{i}$, und $P_{j}$ im endlichen Graphen. 
Der endliche Graph, den man auf diese Weise erhält, wird Quotientengraph (QG) genannt, weil die Beziehung zwischen periodischem Netz und Quotientengraph an die Beziehung zwischen Gruppe und Quotientengruppe erinnert [Chung, Hahn, Klee, 1984]. Abb. 2.4 zeigt ein periodisches Netz (Sechsecknetz), die gitterartige Anordnung der einzelnen Komponenten und seinen Quotientengraphen.
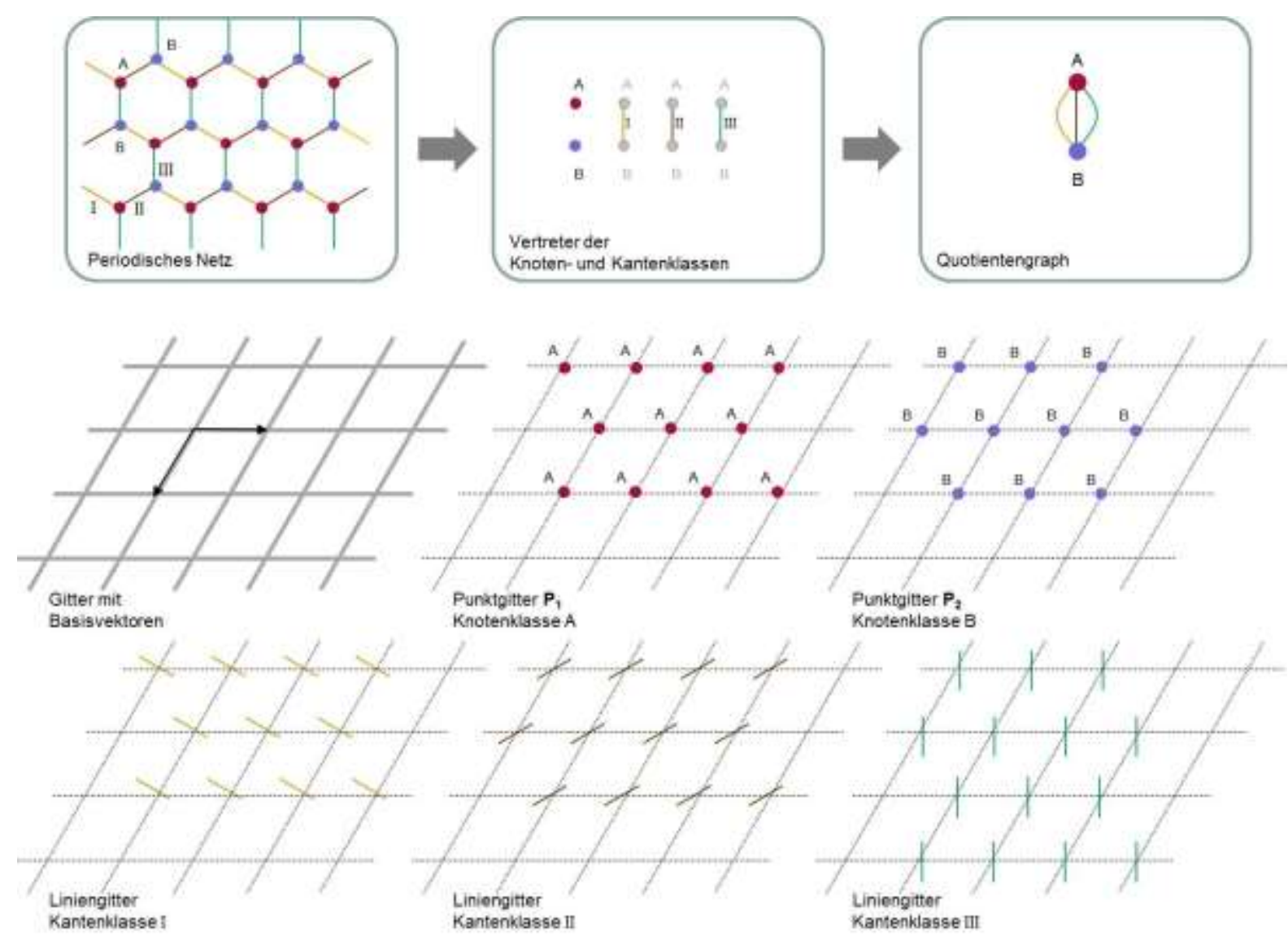

Abb. 2.4 Periodisches Netz mit seinen Punkt- und Liniengittern, und sein Quotientengraph. Sämtliche Elemente des Quotientengraphen sind im periodischen Netz gitterartig angeordnet und bilden dann die einzelnen Elementeklassen (Knoten- und Kantenklassen).

Um ein n-periodisches Netz durch einen Quotientengraphen geringstmöglicher Ordnung darzustellen, wird eine primitive Elementarzelle (Repeatable Unit = Wiederholungseinheit) ermittelt, welche durch $n$ Basisvektoren $(\mathrm{a}, \mathrm{b}, \ldots)$ aufgespannt wird. Elementarzelle und Basisvektoren sind im Netz so zu wählen, dass kein Knoten mehreren Zellen angehört.

Die Zellen werden entsprechend ihrer relativen Position zueinander im Netz mit $\mathrm{n}$ Ziffern (pro Basisvektor eine Ziffer) bezeichnet. Zelle 00 wird mit Zelle 21 zur Deckung gebracht, wenn sie um den Vektor $2 \mathrm{a}+\mathrm{b}$ verschoben wird. Die Angabe der Position eines Knotens entspricht seiner Zellzugehörigkeit (Knoten A(32) liegt in Zelle 32).

Die in einer primitiven Elementarzelle enthaltenen Knoten und Kanten bilden die Ausgangsstruktur des Quotientengraphen. Kanten, die die Knoten von benachbarten Zellen miteinander verbinden, werden ergänzt und indiziert.

Die Indizierung einer solchen Kante beschreibt die Translationsaktion, die die beiden Zellen, zu denen die beiden (durch diese Kante verbundenen) Knoten gehören, zur Deckung bringen. 
Eine Kante, die z. B. Knoten A in Zelle 00 mit Knoten B in Zelle 10 verbindet, wird mit „10“ indiziert und mit einem Pfeil in entsprechender Richtung (von A nach B) versehen. (vgl.

Sechsecknetz in Abb. 2.5 oben links). Dieselbe Kante könnte stattdessen mit einer Indizierung von ,-1 0“ mit einem Pfeil in entgegengesetzte Richtung (von B nach A) versehen werden.

Bei indizierten Schleifen kann auf den Pfeil verzichtet werden, denn der Indizierung einer Schleife im Quotientengraphen entsprechen im Netz zwei Verbindungen in entgegengesetzte Richtungen: z. B. bedeutet die Schleife 10 (in Abb. 2.5 oben rechts), dass Knoten A(00) im Quadratnetz mit den beiden Knoten A(10) und A(-1 0) verbunden ist.

Die Quotientengraphen sind ungerichtete Graphen; das heisst, die hier verwendeten Pfeile ordnen der Kante keine Vorzugsrichtung zu, sondern sie geben im Zusammenhang mit der Indizierung die relative Lage der Zellen ihrer Nachbarknoten an [Chung, Hahn, Klee, 1984].

Die Knotenklassen benachbarter Knoten im Netz entsprechen im Quotientengraphen (QG) benachbarten Knoten. Ein indizierter Quotientengraph kann als eine topologische Elementarzelle eines periodischen Netzes angesehen werden.

Während die Netze schlichte Graphen sind, können die dazugehörigen Quotientengraphen Schleifen oder Mehrfachkanten besitzen (Abb. 2.5).

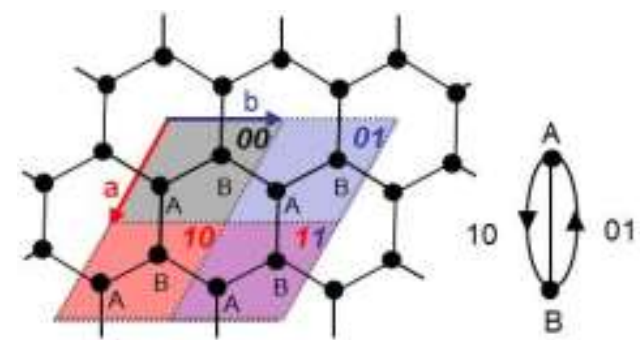

Sechsecknetz

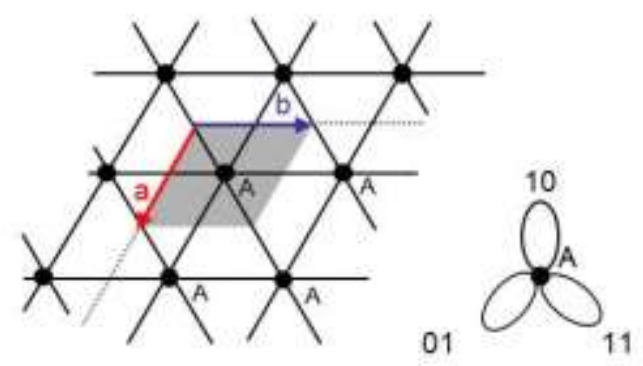

Dreiecknetz

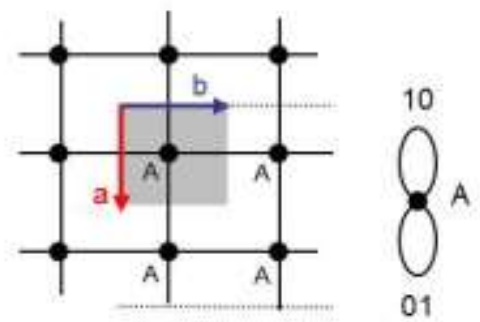

Quadratnetz

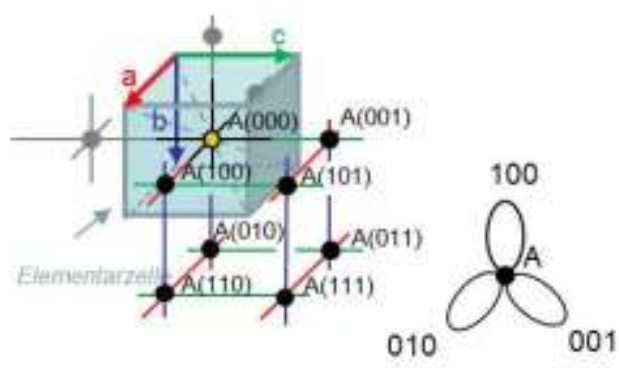

Kubisches Netz

Abb. 2.5 Netze und ihre Quotientengraphen. Das „Sechsecknetz“ hat QG mit Dreifachkante (bei Wahl einer rechteckigen Elementarzelle, die bei diesem Netz keine primitive Elementarzelle ist, entsteht ein anderer endlicher QG, siehe Fig. 3.7 in [Sunada, 2013]); das "Quadratnetz" hat QG mit zwei Scheifen; das „Dreiecknetz“ und das „kubische Netz“ haben gleiche Quotientengraphen, die jedoch unterschiedlich indiziert sind. Im Dreiecknetz ist der Ausgangsknoten $\mathrm{A}(00)$ über die sechs Kanten 10 (a), -10 (-a), 01 (b), 0 -1 (-b), 11 (ab), und -1 -1 (-a-b) mit seinen sechs Nachbarknoten verbunden. Im kubischen Netz ist der in der Mitte der Elementarzelle 000 liegende Knoten A gelb gefärbt, und bei acht seiner Knoten ist die Zellzugehörigkeit angegeben. 
Klassifizierungsmerkmale eines Quotientengraphen sind u. a. seine Ordnung und die Knotengrade seiner einzelnen Knoten [Harary, 1969]. Die Ordnung ist durch die Anzahl der Knoten gegeben, und der Knotengrad gibt die Anzahl der von ihm ausgehenden (der mit ihm inzidierenden) Kanten an. Schleifen an einem Knoten werden doppelt gezählt. So hat der Knoten des Quotientengraphen des Dreiecknetzes aufgrund seiner drei Schleifen den Knotengrad 6 (Abb. 2.6).

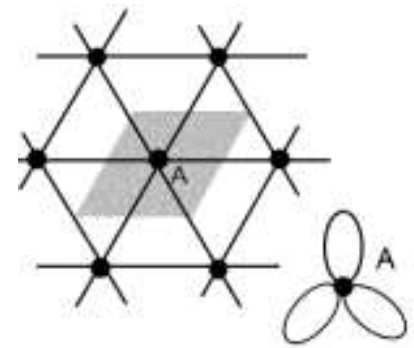

Ordnung: 1 Knotengrad: 6

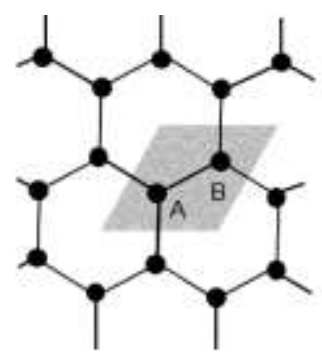

Ordnung: 2 Knotengrad: 3

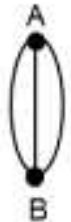

Ordnung: 2

Knoten A: Knotengrad 9 Knoten B: Knotengrad 3

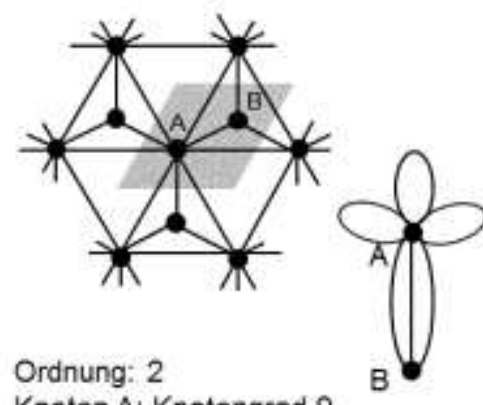

Abb. 2.6 Ordnung und Knotengrad. Die Knotenklassen eines periodischen Netzes können unterschiedliche Knotengrade haben (rechte Skizze).

In Abb. 2.7 sind die Quotientengraphen einiger Kristallstrukturen gezeigt.
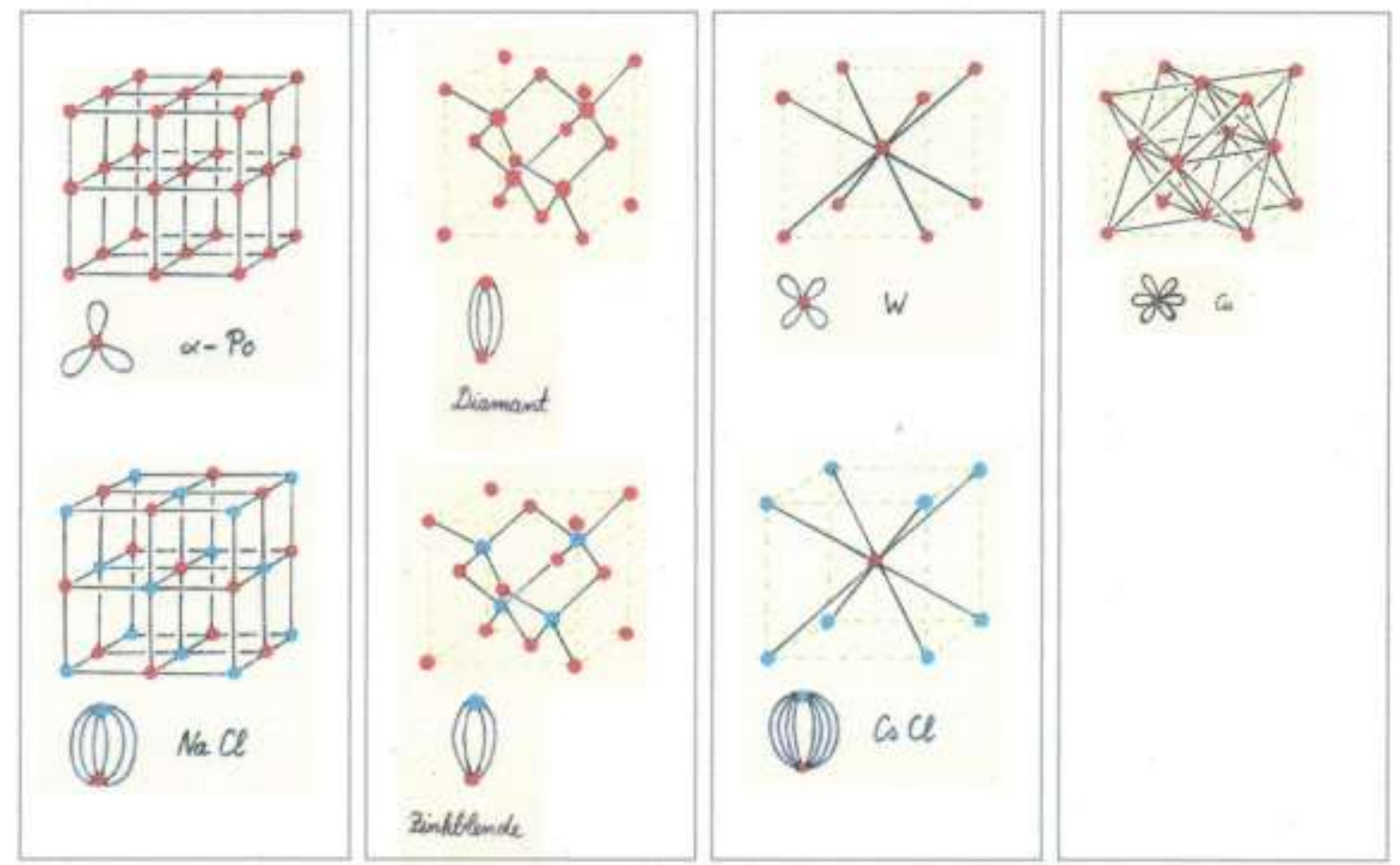

Abb. 2.7 Kristallstrukturen und die dazugehörigen Quotientengraphen [Beukemann, 1989, 1990, Folien für Vorträge]. Die vergleichbaren Strukturen von Diamant und Zinkblende haben gleiche Quotientengraphen, die von $\mathrm{NaCl}$ und $\alpha$-Polonium dagegen nicht. 


\subsection{Kreisklassen}

Netze besitzen aufgrund des für Netze geforderten dreifachen Zusammenhangs Kreise. Sind zwei Kreise translatorisch gleichwertig, so gehören sie derselben Kreisklasse an [Beukemann, 1989]. Die zwei in Abb. 2.8 dargestellten blauen Kreise können durch Translation (Verschiebung) zur Deckung gebracht werden, und sind somit translatorisch gleichwertig. Um ein blaues Dreieck mit dem roten zur Deckung zu bringen, ist neben einer Translation eine Rotation (Drehung) nötig. Das rote Dreieck ist zwar symmetrisch gleichwertig, jedoch nicht translatorisch gleichwertig zu einem der blauen Dreiecke, und gehört somit einer anderen Kreisklasse an. Im Dreiecknetz gibt es zwei Kreisklassen mit Kreisen der Länge 3.

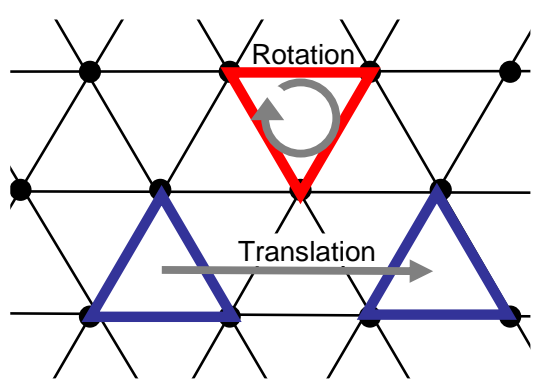

Abb. 2.8 Zwei Kreisklassen der Länge 3 im Dreiecknetz. Ein blauer Kreis kann durch eine Translation nicht mit einem roten zur Deckung gebracht werden.

Das Kreisklassenspektrum gibt an, wie viele Kreisklassen mit welcher Länge ein Netz hat. Lässt man Kreise beliebiger Länge zu, so besitzt ein n-periodisches Netz $(n \geq 2)$ unendlich viele Klassen translatorisch gleichwertiger Kreise [Beukemann, 1989]. Kreise der Länge 1 und 2 gibt es gemäß Definition nicht. Diese Tatsache wird durch das Symbol '-' repräsentiert.

Das Kreisklassenspektrum des Dreiecknetzes bis zur Länge 6 lautet: $\{-,-, 2,3,6,15\}$. Abb. 2.9 zeigt Repräsentanten der Kreisklassen dieses Netzes bis zur Länge 6 .

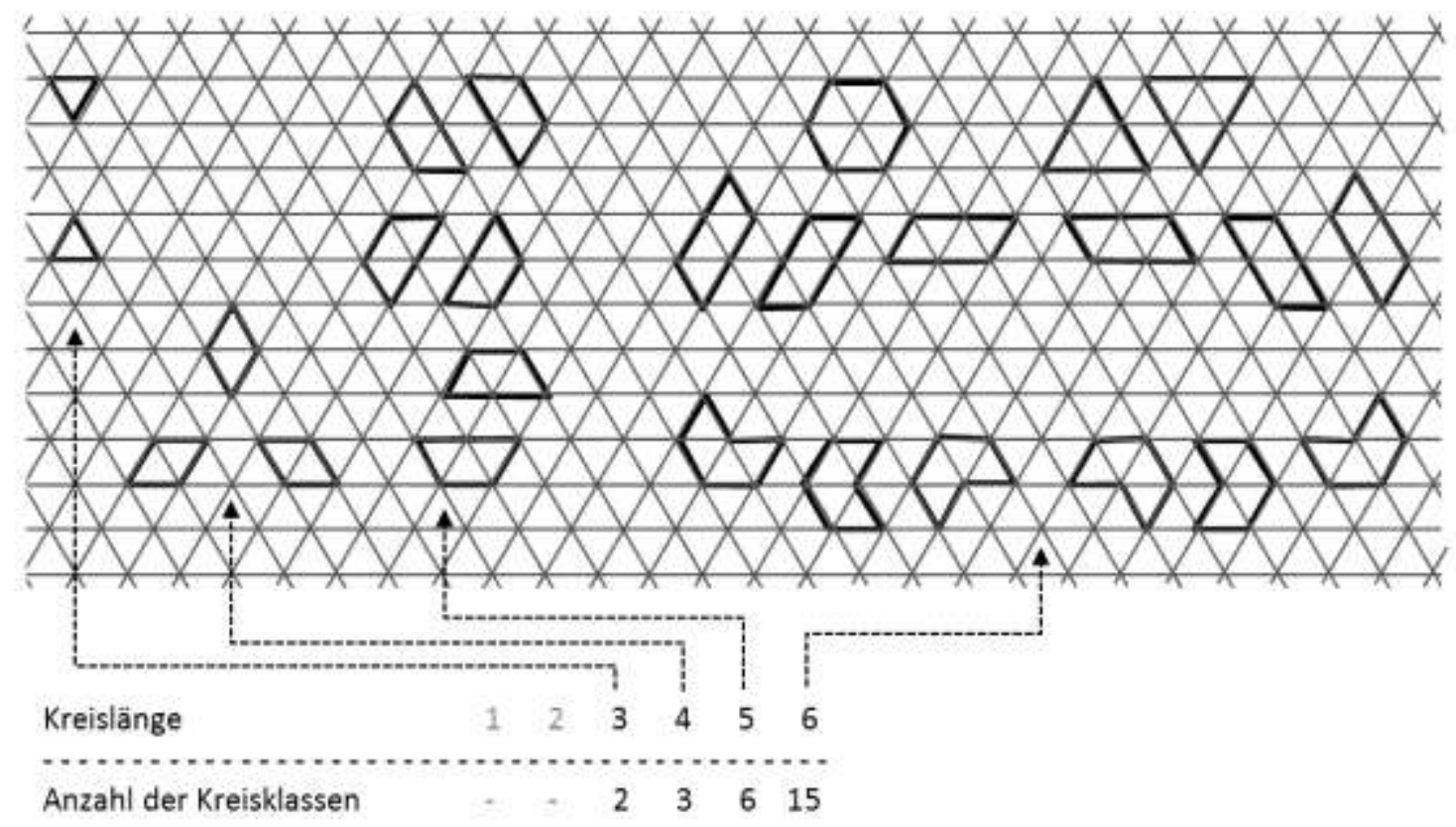

Abb. 2.9 Dreiecknetz: Repräsentanten seiner Kreisklassen bis zur Länge 6. 
Für das Quadratnetz sind hier nur die Vertreter aller symmetrisch (!) gleichwertigen Kreise gezeigt. Die Anzahl seiner Kreisklassen erhält man daraus durch Multiplikation mit dem Quotienten aus der Ordnung der Punktgruppe des Netzes und der Ordnung der Punktgruppe des Kreises (Abb. 2.10 und Abb. 2.11).

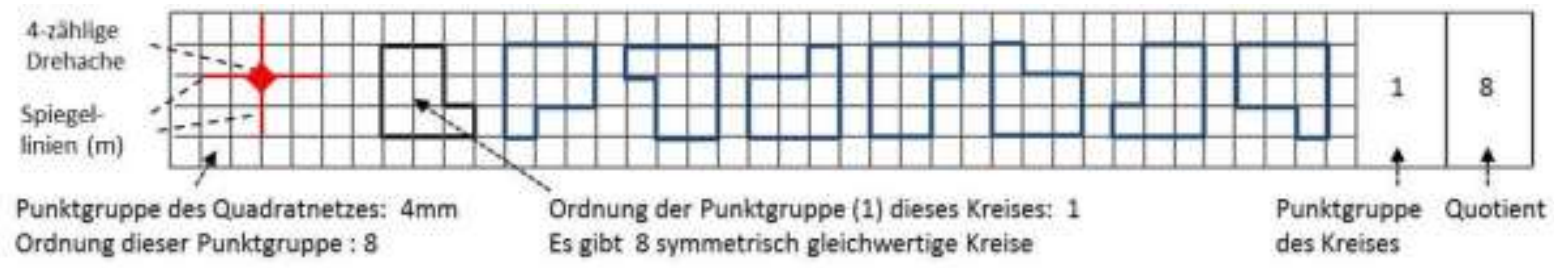

Abb. 2.10 Symmetrisch gleichwertige Kreise. Die acht abgebildeten Kreise sind Vertreter von acht Kreisklassen der Länge 12. In Abb. 2.11 ist von diesen nur der schwarze Kreis aufgeführt.

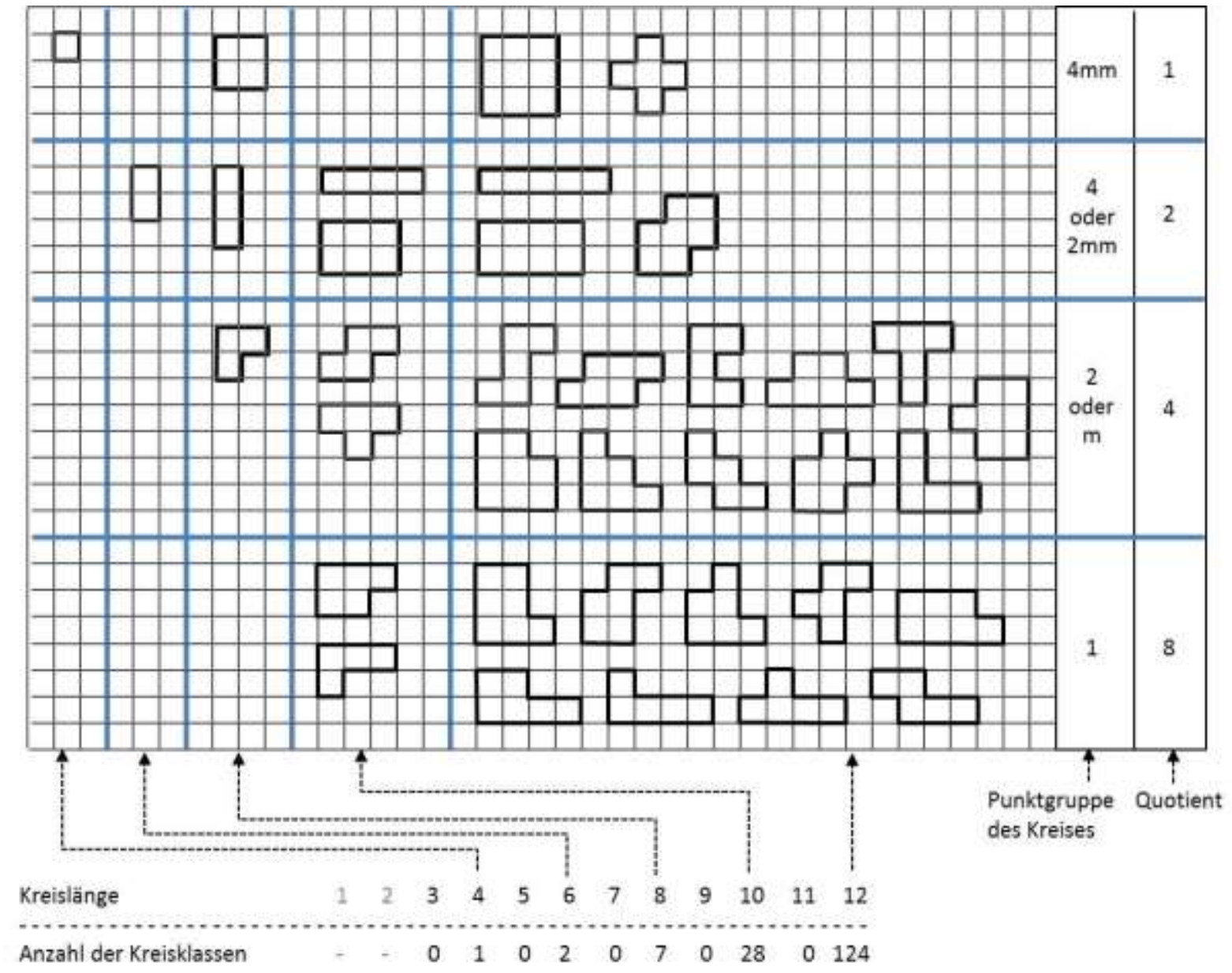

Abb. 2.11 Quadratnetz: Repräsentanten symmetrisch gleichwertiger Kreise bis zur Länge 12 . Die 124 Kreisklassen mit Kreisen der Länge 12 ergeben sich aus der 5. Spalte wie folgt:

2 (Figuren) $x 1$ (Quotient) $+3 \times 2+11 \times 4+9 \times 8=124$

Das Kreisklassenspektrum des Quadratnetzes bis zur Länge 12 lautet somit: $\{-,-, 0,1,0,2,0,7,0,28,0,124\}$.

Kreisklassenspektren (cycle class sequences) verschiedener bekannter Kristallstrukturen wie Quarz, Feldspat, ... sind in [Beukemann, Klee, 1994] aufgeführt. 


\subsection{Minimalnetze}

Minimalnetze [Beukemann, Klee, 1992 und Beukemann 1989] sind Netze, bei denen das Weglassen einer (beliebig wählbaren) Kante samt der dazu translatorisch gleichwertigen zum Zerfall des Netzes führt. Die translatorische Äquivalenz, eine Eigenschaft der Einbettung, wird in dieser Arbeit auch auf abstrakte Netze bezogen.

Zur Erläuterung zwei Beispiele:

- Dreiecknetz: Flächenaufteilung (Tiling, Kachelung) durch Dreiecke

Wird beim „Dreiecknetz“ eine Kantenklasse (in der Abb. 2.12 gestrichelt) gelöscht, so bleibt ein zusammenhängender Graph übrig, nämlich ein „deformiertes“ Quadratnetz. Somit ist das Dreiecksnetz kein Minimalnetz.

- Sechsecknetz: Flächenaufteilung (Tiling, Kachelung) durch Sechsecke

Das Löschen einer beliebigen Kantenklasse beim „Sechsecknetz“ führt dagegen zum Zerfall dieses Netzes (Abb. 2.12 rechts unten). Damit erfüllt das Sechsecknetz die Bedingung für ein Minimalnetz.
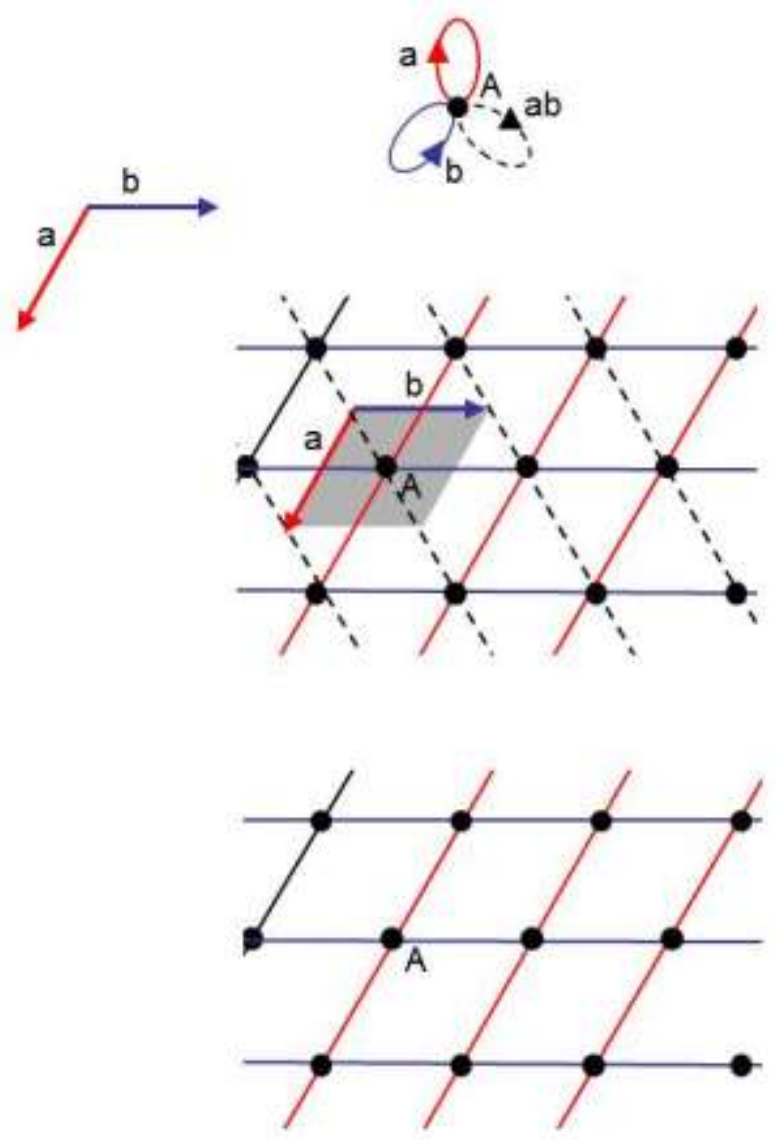
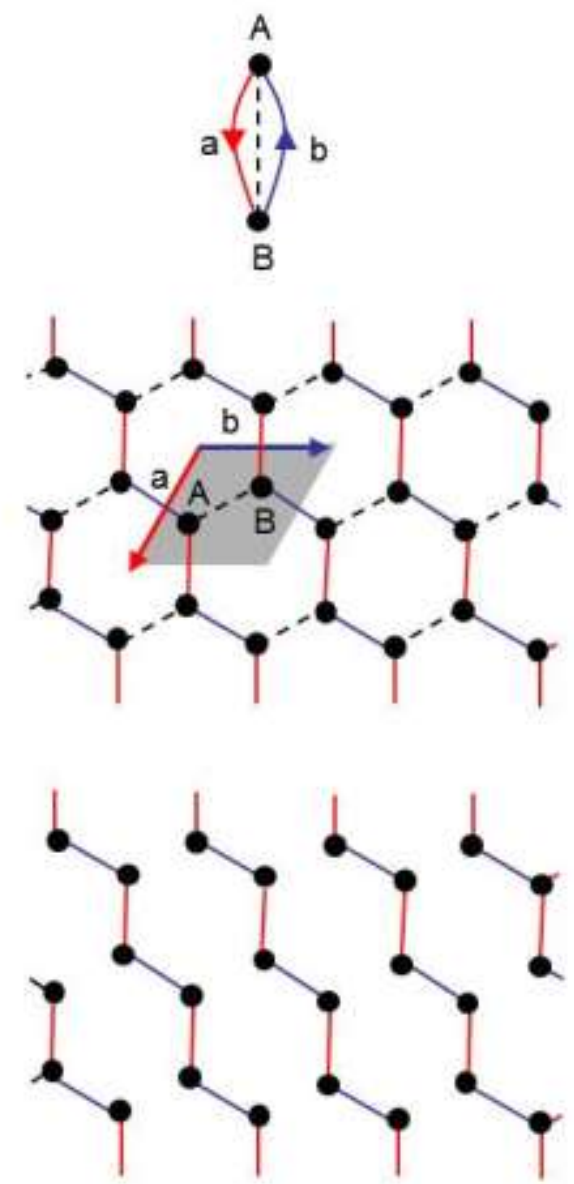

Abb. 2.12 Dreieck- und Sechsecknetz. Minimalnetz: ja oder nein? Die Kanten wurden hier vereinfacht mit a (anstatt 10), b (anstatt 01) und ab (anstatt 11) indiziert. Das Dreiecknetz ist kein Minimalnetz, das Sechsecknetz dagegen schon. 
Zwischen einem Minimalnetz und seinem dazugehörigen Quotientengraphen besteht eine 1:1-Relation. Somit kann bei Minimalnetzen auf die Indizierung seiner Kanten verzichtet werden [Beukemann, Klee, 1992 und Beukemann 1989].

Um n Knoten miteinander zu verbinden, sind n-1 Kanten notwendig. Diese n Knoten und n-1 Kanten bilden einen „Baum“. Die übrigen Kanten „erzeugen“ die Periodizitäten.

Periodizität $=$ Anzahl der Kanten $-($ Anzahl der Knoten -1$) \quad$ [Beukemann, Klee 1992]

Ein Minimalnetz mit 6 Knotenklassen und 9 Kantenklassen ist also vierperiodisch: 9-(6-1) = 4

Die Quotientengraphen der Minimalnetze wurden in [Beukemann, Klee 1992] entsprechend ihrer Ordnung und ihrer Knotengrade klassifiziert und dann durchnummeriert:

- zuerst die Ordnung (Anzahl der verschiedenen Knotenklassen)

- dann in Klammern die Knotengrade der einzelnen Knotenklassen, wobei z. B. $(3,3,3,3,4)$ verkürzt dargestellt wird als $\left(3^{4}, 4\right)$.

- schließlich werden unterschiedliche Quotientengraphen gleicher Ordnung und Knotengrade durchnummeriert (beginnend mit 1). Gibt es zu einer Kombination aus Ordnung und Knotengraden nur einen Quotientengraphen, so erhält er die Nummer 1.

Für acht der 15 dreiperiodischen Minimalnetze führen Bonneau et al. bekannte Kristallstrukturen als Beispiele auf. Symbole, welche Raumaufteilungen beschreiben, die nach einem - diese Struktur repräsentierenden - Kristall benannt werden, ordnen sie für diese Netze den BK-Symbolen zu (Tab. 2.1) [Bonneau, Delgado-Friedrichs, O’Keeffe, Yaghi, 2004].

$\begin{array}{lclcc}\text { Net } & \text { BK } & \text { Symmetrie } & \text { Coord. } & {[\ldots]} \\ \ldots & & & & \\ \text { dia } & 2(4) 1 & \mathrm{Fd} 3 \mathrm{~m} & 4 & {[\ldots]} \\ \ldots & & & & \\ \text { srs } & 4(3) 1 & \mathrm{I}_{1} 32 & 3 & {[\ldots]} \\ \ldots & & & & \\ \text { 'BK' refers to the symbol of Beukemann \& Klee (1992) }\end{array}$

Tab. 2.1 Ausschnitt aus Table 1 [Bonneau, Delgado-Friedrichs, O'Keeffe, Yaghi, 2004]

Die Ableitungen dieser Symbole (z. B. dia für Diamant, und srs für $\mathrm{SrSi}_{2}$ ) sind in [DelgadoFriedrichs, O'Keeffe, Yaghi, 2007] erläutert. Für die in der vorlegenden Arbeit betrachteten Netze, die vierperiodischen Minimalnetze, wird die BK-Nomenklatur von [Beukemann, Klee 1992] verwendet. Ein Beispiel:

Minimalnetz $5\left(3^{4}, 4\right) 7$

- Ordnung 5, d. h. fünf verschiedene Knotenklassen

- vier Knoten haben den Knotengrad 3, ein Knoten hat den Knotengrad 4

- von den Netzen, die die zwei oberen Kriterien erfüllen, wird das vorliegende als Nummer 7 benannt.

Eine vollständige Übersicht der 2-, 3- und 4-periodischen Minimalnetze befindet sich im Anhang. 
Um in einem Kreis eines Minimalnetzes wieder zum Ausgangsknoten zurückzukommen, muss jede an diesem Kreis beteiligte Kante geradzahlig oft durchlaufen werden [Beukemann, 1989]. Somit gibt es in Minimalnetzen nur Kreisklassen mit geradzahliger Länge, beginnend mit der Kreislänge 4. In den Kreisklassenspektren dieser Arbeit werden, da die betrachteten Netze allesamt Minimalnetze sind, von nun an nur noch die Kreisklassen geradzahliger Kreislänge ab der Kreislänge 4 aufgelistet.

Für das Minimalnetz 1(4)1 (Quadratnetz) werden z. B. im Kreisklassenspektrum anstelle aller Längen $\{-,-, 0,1,0,2,0,7,0,28,0,124, \ldots\}$ nur geradzahlige Längen ab Länge 4 aufgeführt. Ein Kreisklassenspektrum mit der Zahlenreihe 122728124 bedeutet also:

- eine Kreisklasse mit Kreisen der Länge 4

- zwei Kreisklassen mit Kreisen der Länge 6

- sieben Kreisklassen mit Kreisen der Länge 8

- 28 Kreisklassen mit Kreisen der Länge 10

- 124 Kreisklassen mit Kreisen der Länge 12

Abb. 2.13 zeigt die drei zweiperiodischen Minimalnetze (Ausschnitte) mit ihren Quotientengraphen (jeweils oben links) und ihren Kreisklassenspektren mit der Anzahl der Kreisklassen der Längen 4, 6, 8, 10, 12 (jeweils unten rechts).

Kreislänge: $\quad 4 \quad 6 \quad 8 \quad 8 \quad 10 \quad 12$

$\begin{array}{llllll}\text { Minimalnetz 1(4)1: } & 1 & 2 & 7 & 28 & 124\end{array}$

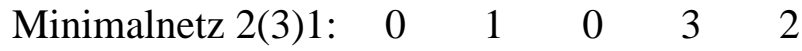

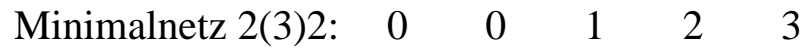

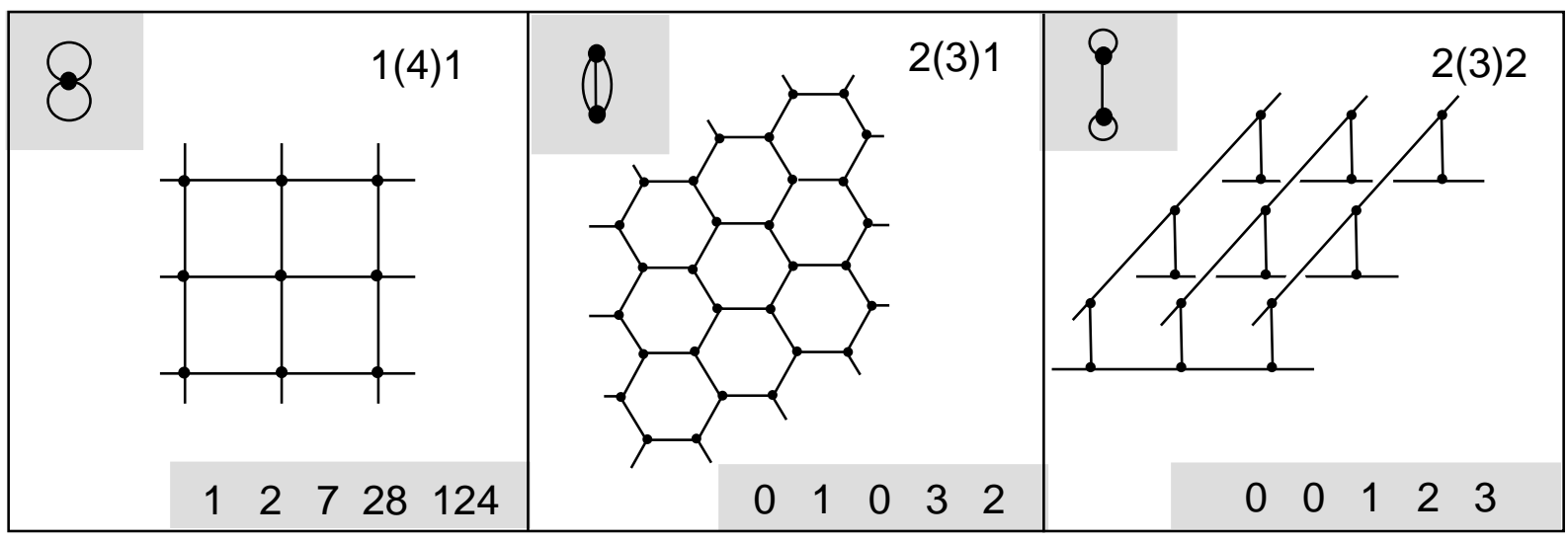

\section{Abb. 2.13 Die 3 zweiperiodischen Minimalnetze}

Alle zwei-, drei- und vierperiodischen Minimalnetze sind anhand ihrer Kreisklassenspektren unterscheidbar, sofern die charakterisierenden Parametertupel in ausreichender Länge vorliegen. Ihre Kreisklassenspektren sind im Anhang unter dem jeweiligem Netz aufgeführt. Die Bestimmung der Parametertupel erfolgte mit dem Programm „Kreise“ [Beukemann, 1989] unter Einhaltung obiger Bedingung. 
Eigenschaften der dreiperiodischen Minimalnetze sind in Tab 2.2 aufgelistet, ergänzt um neuere Ergebnisse aus [Bonneau, Delgado-Friedrichs, O’Keeffe, Yaghi, 2004].

\begin{tabular}{|c|c|c|c|c|}
\hline \multicolumn{2}{|c|}{ [Beukemann, Klee, 1992] } & \multicolumn{3}{|c|}{ [Bonneau et al., 2004] } \\
\hline Q-graph & $\begin{array}{l}\text { Kreisklassen } \\
\begin{array}{llllll}4 & 6 & 8 & 10 & 12 & 14\end{array} \quad \text { Netz }\end{array}$ & Tiles & Symmetry & Net \\
\hline 1(6)1 & 322207 & & $P m \overline{3} m$ & pcu \\
\hline $2(4) 1$ & 044648 & & $F d \overline{3} m$ & dia \\
\hline $2(4) 2$ & 02832 & & $\mathrm{PH}_{2} / \mathrm{mmc}$ & cds \\
\hline $2(3,5) 1$ & 044954 & & Pб̄m2 & hms \\
\hline $2(3,5) 2$ & 12948 & & & *) \\
\hline 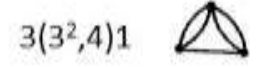 & $\begin{array}{lllll}0 & 0 & 5 & 221\end{array}$ & & $\overline{1} m 2$ & $\mathrm{tfa}$ \\
\hline $3\left(3^{2}, 4\right) 2$ & $\begin{array}{llllll}0 & 1 & 0 & 6 & 11\end{array}$ & & & *) \\
\hline $3\left(3^{2}, 4\right) 3$ & $\begin{array}{llllll}0 & 0 & 3 & 6 & 17\end{array}$ & & $\mathrm{Cmmm}$ & $\mathrm{tfc}$ \\
\hline $3\left(3^{2}, 4\right) 4$ & $\begin{array}{lllll}0 & 0 & 2 & 4 & 7\end{array}$ & & & *) \\
\hline $3\left(3^{2}, 4\right) 5$ & $\begin{array}{llllll}0 & 1 & 1 & 3 & 13\end{array}$ & & & *) \\
\hline $4(3) 1$ & $\begin{array}{lllll}0 & 0 & 0 & 6 & 0\end{array}$ & & 14,32 & srs \\
\hline $4(3) 2 \quad \square$ & $\begin{array}{lllll}0 & 0 & 0 & 4 & 2\end{array}$ & & $14_{1} /$ amd & ths \\
\hline $4(3) 3$ & $\begin{array}{lllll}0 & 0 & 1 & 0 & 3\end{array}$ & & & $\left.{ }^{*}\right)$ \\
\hline $4(3) 4$ & $\begin{array}{llllll}0 & 0 & 0 & 0 & 3 & 6\end{array}$ & & & *) \\
\hline $4(3) 5$ & $\begin{array}{lllll}0 & 0 & 0 & 2 & 2\end{array}$ & & & *) \\
\hline
\end{tabular}

Tab. 2.2 Die 15 dreiperiodischen Minimalnetze. 5. - 7. Spalte (inklusive der acht Abbildungen unter „Tiles“) aus [Bonneau, Delgado-Friedrichs, O’Keeffe, Yaghi, 2004].

*) Diese sieben Netze werden als Netze mit Kollisionen (nets with collisions) [Bonneau et al., 2004] und als instabile Netze (instable nets) [Eon, 2011] bezeichnet. 


\section{Substrukturen vierperiodischer Minimalnetze}

In der Kristallchemie wird die Topologie von Kristallstrukturen oft dadurch analysiert, indem diese in ein- oder zweiperiodische Strukturen zerlegt werden [Eon, Proserpio, Blatov, 2012]. In Abb. 3.1 sind zwei einfache Beispiele von Substrukturen gezeigt.

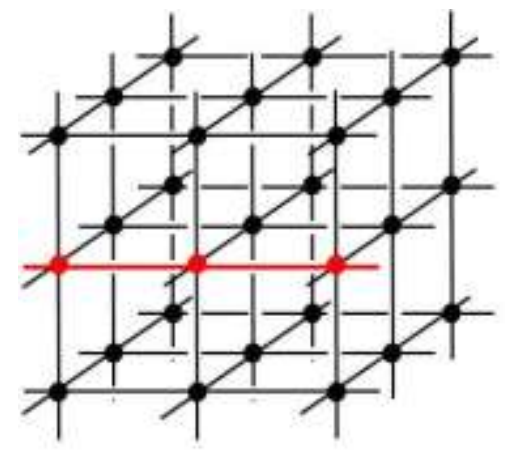

1-periodische Substruktur

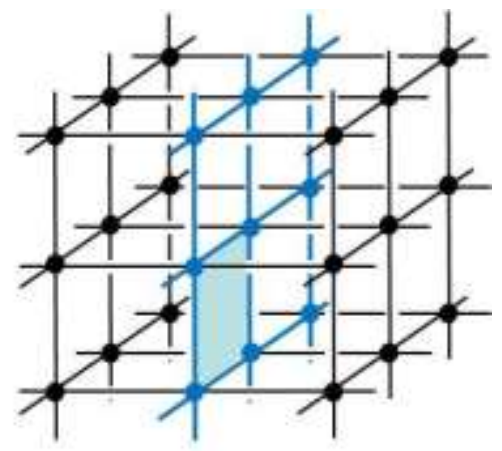

2-periodische Substruktur

Abb. 3.1 Einfache Substrukturen eines dreiperiodischen Gitters. Links (rot gekennzeichnet) eine 1-periodische Substruktur (Kette), rechts (blau gekennzeichnet) eine 2-periodische Substruktur; hellblau: ein Kreis der Länge 4 dieser 2-periodischen Struktur.

Die Analyse von Substrukturen eignet sich sehr gut, um verschiedene vierperiodische Minimalnetze zu analysieren und zu vergleichen. Abb. 3.2 zeigt dreiperiodische Substrukturen des vierperiodischen Minimalnetzes 1(8)1.
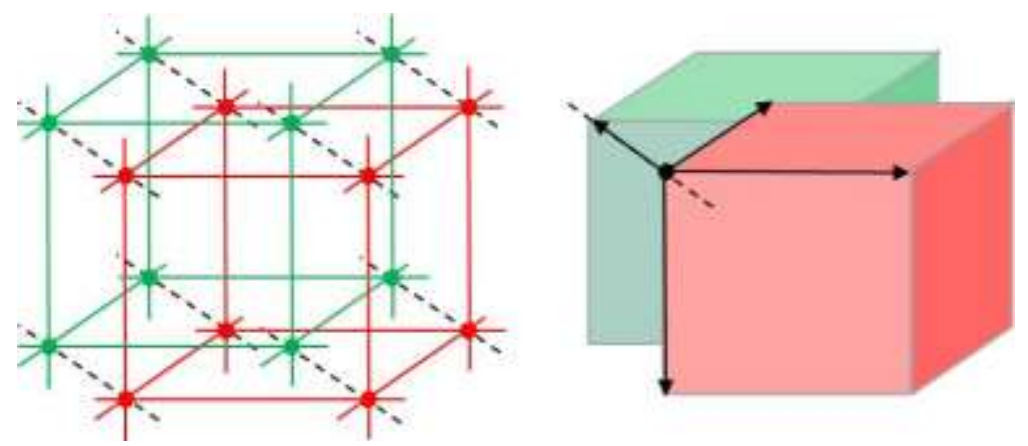

Abb. 3.2 Dreiperiodische Substrukturen. Links: Die kubischen Gitter (rot und blau) sind Substrukturen des vierperiodischen Gitters (vgl. Abb. 1.7), welches dem Minimalnetz 1(8)1 entspricht. Rechts die vier (linear unabhängigen) Basisvektoren dieses Gitters.

Zur Analyse der vierperiodischen Minimalnetze werden folgende Substrukturen betrachtet:

- Bäume: 0-periodische Substrukturen

- Ketten: 1-periodische Substrukturen

- Kreise/Kreisklassen: 2-periodische Substrukturen

- Netze: 2- und 3-periodische Minimalnetze 


\subsection{Bäume: 0-periodische Substrukturen}

Diagramme aller Bäume mit bis zu zwölf Knoten wurden von G. Prins angefertigt [Prins, 1957]. Die Bäume mit bis zu zehn Knoten sind in [Harary, 1969] dargestellt.

Betrachtet werden in dieser Arbeit nur solche Bäume als Substrukturen, die sämtliche Knoten eines Quotientengraphen eines vierperiodischen Minimalnetzes miteinander verbinden.

Daraus ergibt sich:

- Quotientengraphen der vierperiodischen Minimalnetze der Ordnung 2 haben nur einen möglichen Baum, bestehend aus zwei Knoten, die mit einer Kante verbunden werden.

- Auch für die Quotientengraphen der vierperiodischen Minimalnetze der Ordnung 3 lässt sich nur eine einzige Baumstruktur finden. Ein Knoten ist hier grundsätzlich über zwei Kanten mit den zwei anderen Knoten verbunden.

- Unter den Quotientengraphen vierperiodischer Minimalnetze mit vier oder mehr Knoten gibt es unterschiedliche Baumstrukturen (siehe Abb.3.3).

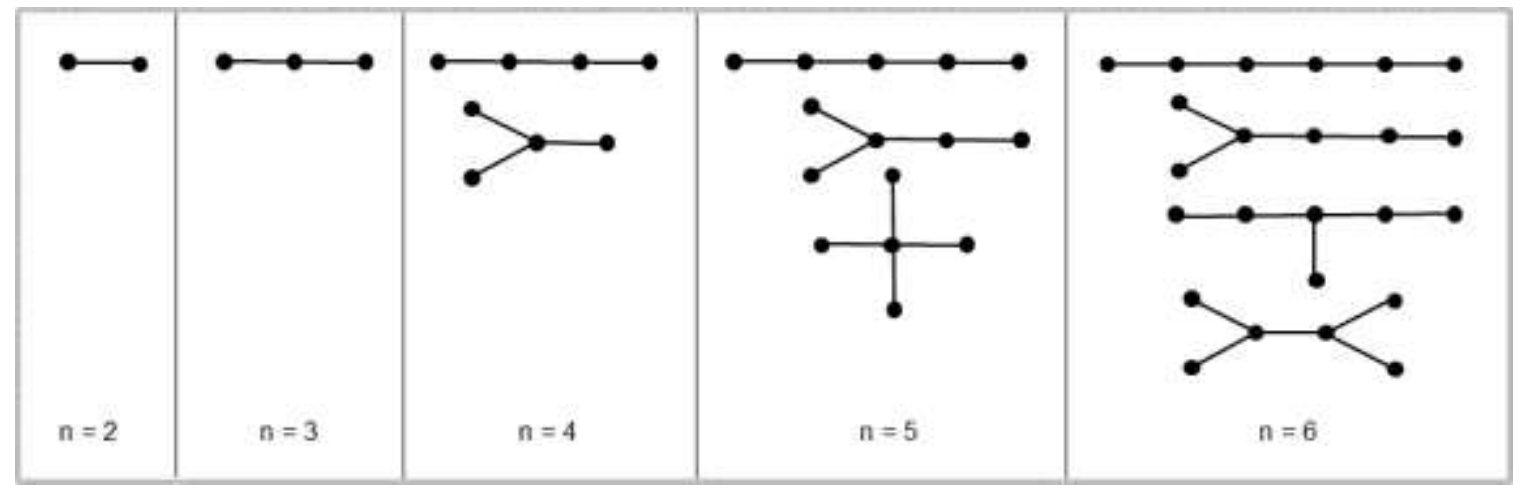

Abb. 3.3 Bäume in den Quotientengaphen 4-periodischer Minimalnetze. Bemerkung zu n=6: um 6 Knoten über eine Baumstruktur zu verbinden, gibt es prinzipiell noch zwei weitere Baumstrukturen, die jedoch in vierperiodischen Minimalnetzen nicht vorkommen können.

In den Abbildungen auf den folgenden Seiten sind die Quotientengraphen gruppiert nach ihren Baumstrukturen. Die Ellipsen in Abb. 3.4 bis 3.6 sind den einzelnen Baumstrukturen zugeordnet. In den Ellipsen sind jeweils die Quotientengraphen abgebildet, in denen diese Baumstrukturen zu finden sind. Bei den Schnittmengen ist mehr als eine Baumstruktur je Graph zu finden. 
In Abb. 3.4 sind oberhalb der gestrichelten Linie die vierperiodischen Minimalnetze der Ordnung 4 des Typs $4\left(3^{3}, 5\right)$, unterhalb diejenigen des Typs $4\left(3^{2}, 4^{2}\right)$ dargestellt.

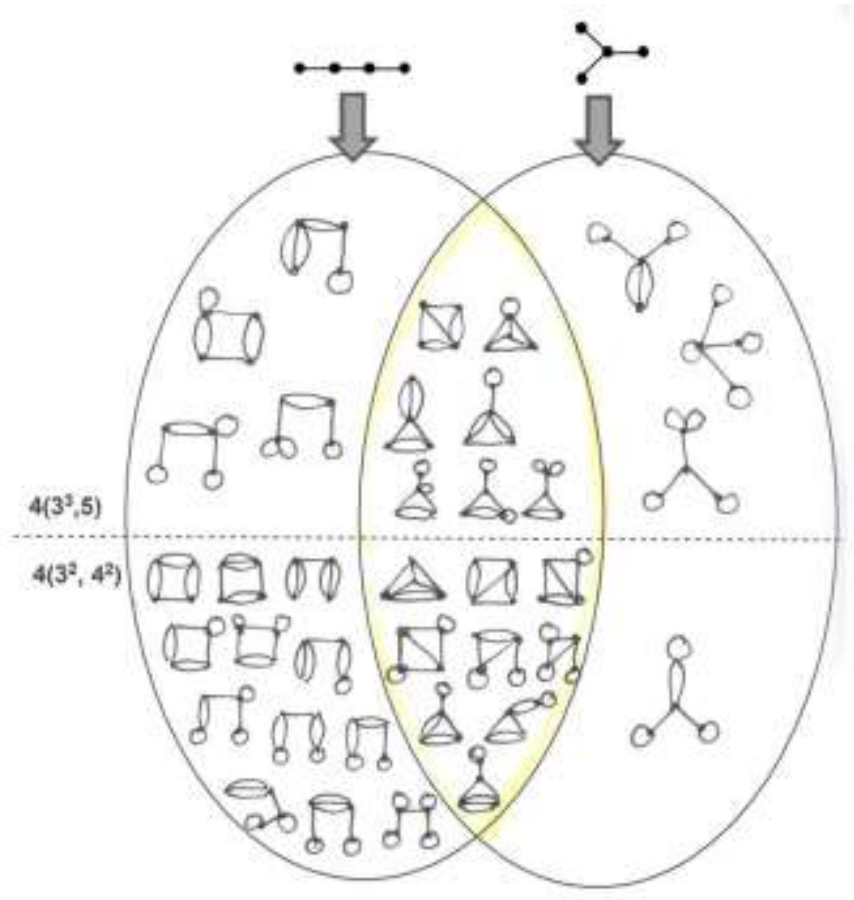

Abb. 3.4 Zuordnung der Quotientengraphen der Ordnung 4 zu den zwei Baumstrukturen. In 16 Graphen (Schnittmenge der beiden Ellipsen) sind beide Strukturen zu finden.

Unter den Quotientengraphen der vierperiodischen Minimalnetze der Ordnung 5 (Abb. 3.5) gibt es drei, bei denen sich alle drei Baumstrukturen bilden lassen (Schnittmenge der drei Ellipsen: Feld „1,2,3“). Die Ellipsen 1,2,3 sind den drei Baumstrukturen zugeordnet.

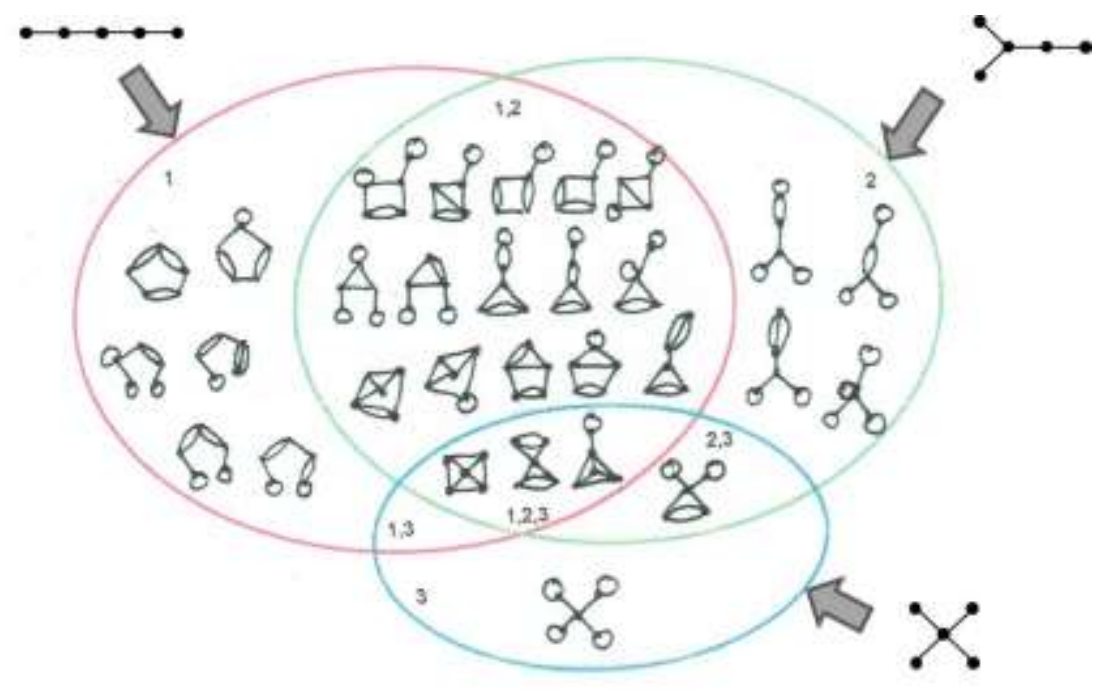

Abb. 3.5 Zuordnung der Quotientengraphen der Ordnung 5 zu den drei Baumstrukturen. Baumstruktur „1“(oben links) in Graphen, die sich in der roten Ellipse befinden. Baumstruktur „2“ (oben rechts): grüne Ellipse, Baumstruktur ,3“ (unten): blaue Ellipse. Die Zahlenangaben in den einzelnen Feldern geben an, welche der drei Baumstrukturen in den Graphen, die diesen Feldern zugeordnet sind, zu finden sind. 
Unter den 17 Quotientengraphen der vierperiodischen Minimalnetze der Ordnung 6 gibt es zwei, bei denen sich alle vier Baumstrukturen bilden lassen; sie sind im Feld ,1,2,3,4“ (Schnittmenge der 4 Ellipsen) dargestellt (Abb. 3.6).

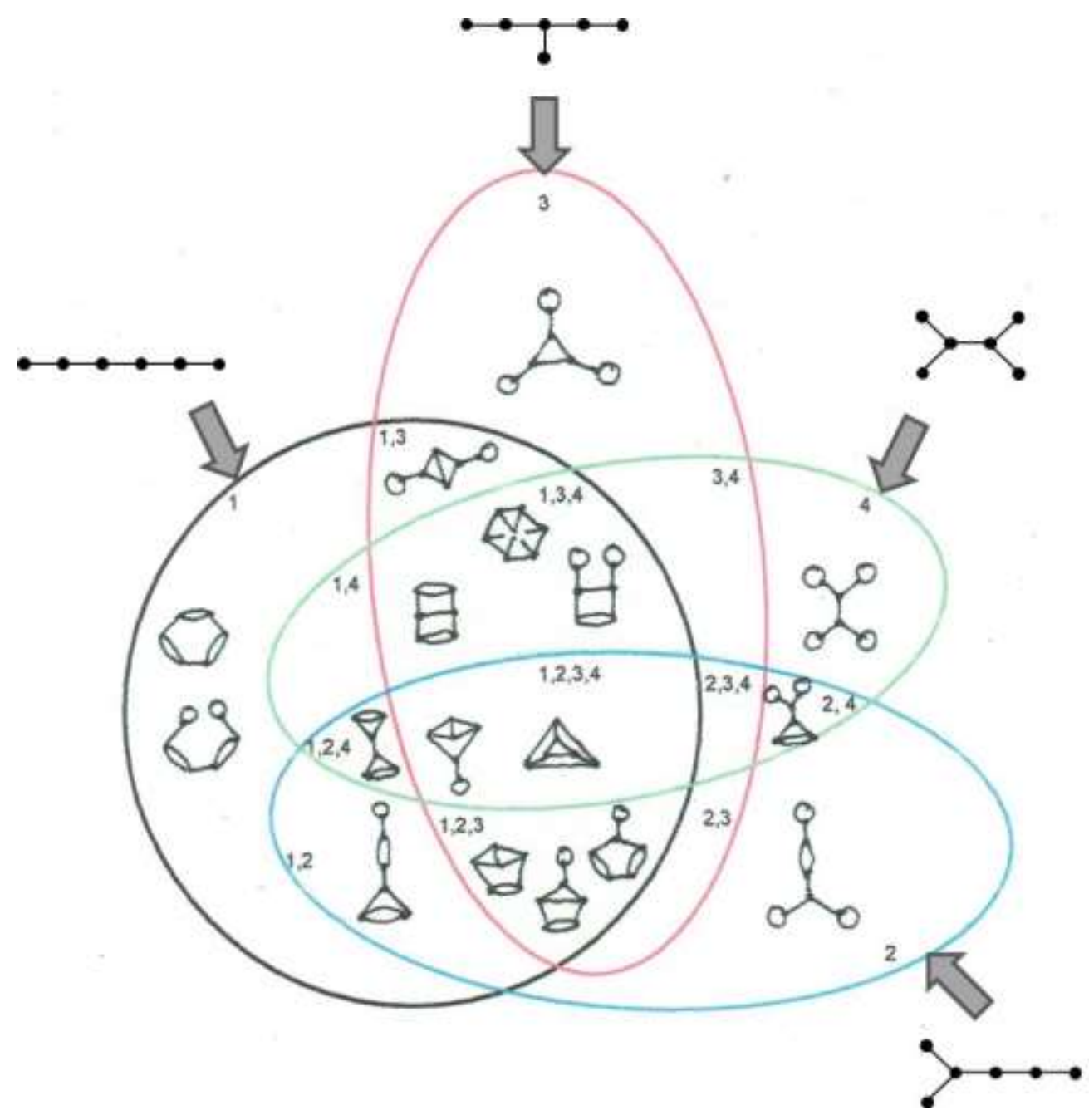

Abb. 3.6 Zuordnung der Quotientengraphen der Ordnung 6 zu den vier Baumstrukturen. Graphen mit Baumstruktur ,1“ in schwarzer Ellipse, mit Baumstruktur ,2“ in blauer Ellipse, mit Baumstruktur ,3“ in roter Ellipse, mit Baumstruktur „4“ in grüner Ellipse. Die Felder „1,4“, „2,3“, $, 2,3,4$ " und ,3,4“ sind unbesetzt. 
Ausgehend von gleichen Baumstrukturen lassen sich bestimmte Netze gut miteinander vergleichen, wie z. B. die in Abb. 3.7 senkrecht untereinander angeordneten Netze $5\left(3^{4}, 4\right) 1$ und $5\left(3^{4}, 4\right) 7$. Analoges gilt für die Netze 6(3)2 und 6(3)14. Die hier jeweils nebeneinander dargestellten Netze $5\left(3^{4}, 4\right) 1$ und 6(3)2 wurden so konstruiert, dass sie sich nur in Bezug auf ihre Baumstruktur unterscheiden. Analoges gilt für die Netze $5\left(3^{4}, 4\right) 7$ und 6(3)14.

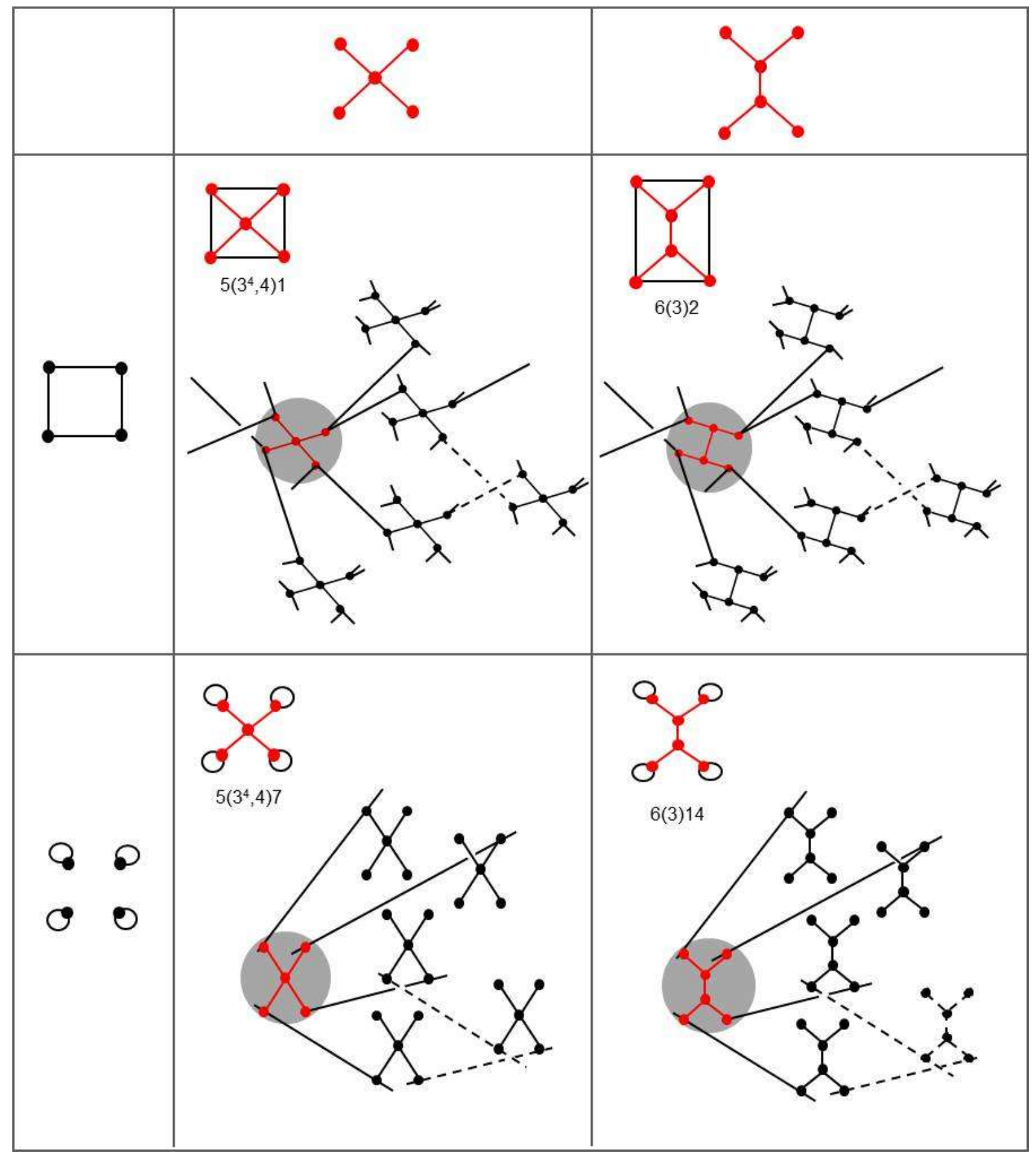

Abb. 3.7 Vergleich von Minimalnetzen anhand gewisser Baumstrukturen. Zwei Baumstrukturen (oben) werden auf unterschiedliche Weise durch jeweils vier (linear unabhängig zu indizierende) Kanten (linke Spalte) ergänzt. In beiden Fällen ist jede dieser Kanten für eine Periodizität verantwortlich. In den vier Netzdarstellungen ist jeweils eine Baumstruktur grau hinterlegt. Bei den paarweise untereinander dargestellten Netzen sind die Bäume auf unterschiedliche Weise verknüpft. Die hier paarweise nebeneinander dargestellten Netze unterscheiden sich bezüglich ihrer Baumstruktur. 


\subsection{Ketten: 1-periodische Substrukturen}

Sind $\mathrm{n}$ Knoten in einem Quotientengraphen über einen Kreis verbunden, so kann daraus eine unendliche Kette als Substruktur gebildet werden. Die Knoten dieser Kette können in einer Linie angeordnet werden. Die Kette ist also eine 1-periodische Substruktur. Ketten sind als graphentheoretischer Begriff in [Thimm, 2008] eingeführt.

In Abb. 3.8 ist jeweils ein Kreis im Quotientengraphen der 1-periodischen Substruktur (Kette) im Minimalnetz gegenübergestellt. Die Anzahl unterschiedlicher Knotenklassen innerhalb einer 1-periodischen Kette wird im folgenden Periodenlänge (PL) genannt.

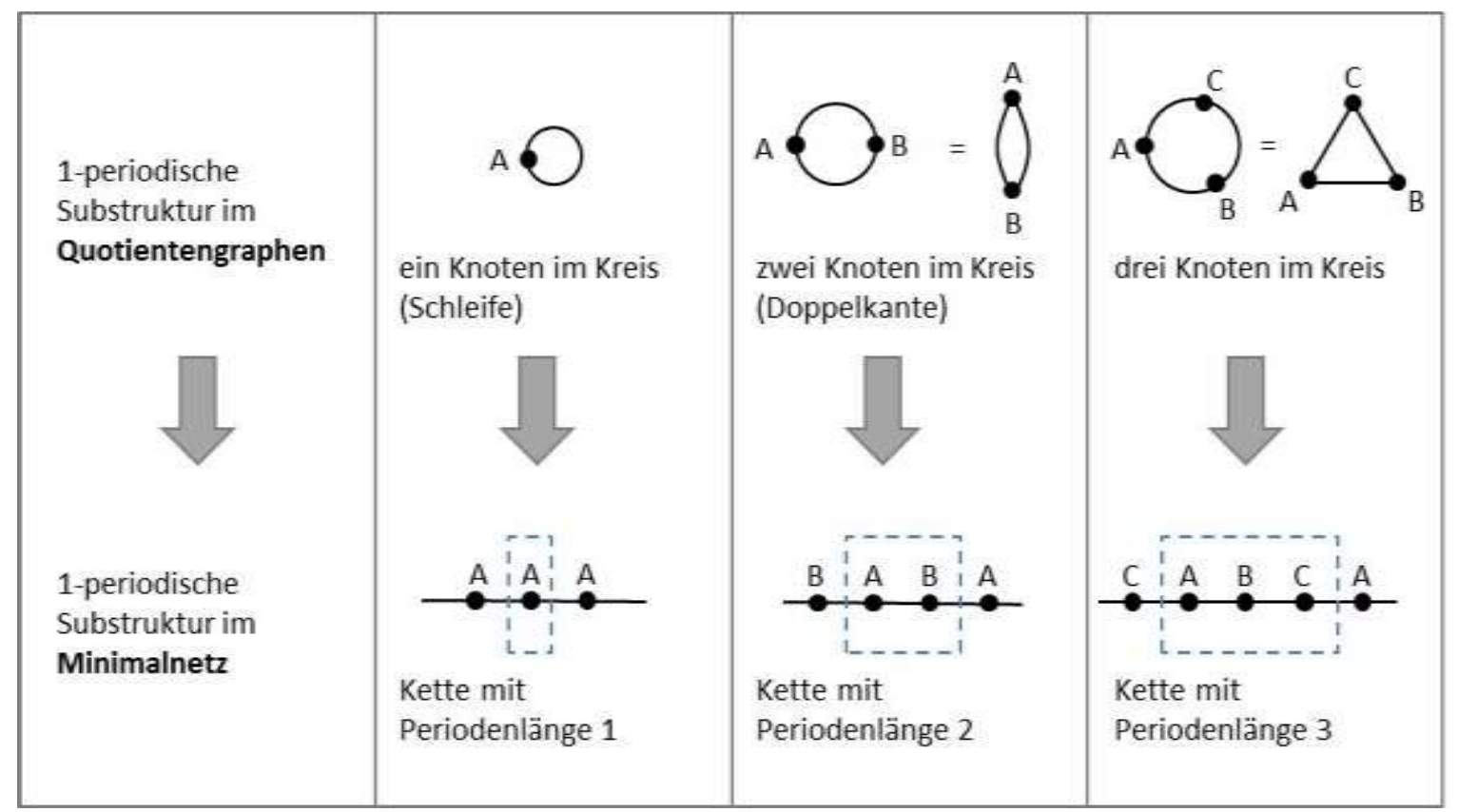

Abb. 3.8 Kreise in Quotientengraphen und ihre Ketten im dazugehörigen Netz

Im Quotientengraphen des vierperiodischen Minimalnetzes 6(3)3 findet man Kreise der Längen 2, 3, 4, 5 und 6, und im dazugehörigen Netz die 1-periodischen Ketten entsprechender Länge (Abb. 3.9).
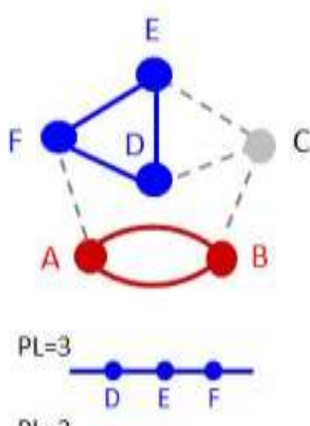

$P L=2$

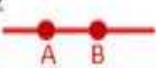

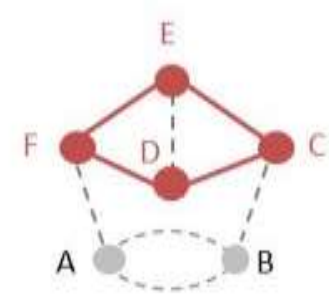

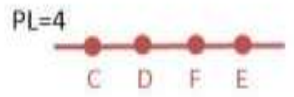

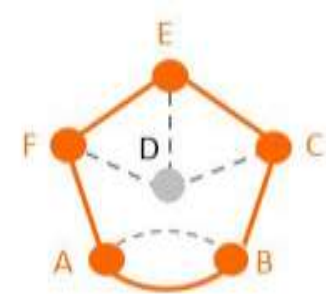

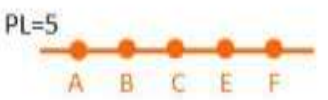

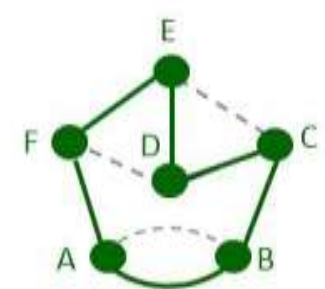

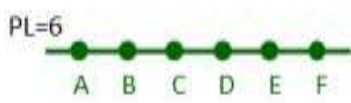

Abb. 3.9 Ketten im Quotientengraphen des vierperiodischen Minimalnetzes 6(3)3. Die Ketten der Periodenlängen 2, 3, 4, 5 und 6 des Netzes werden in seinem Quotientengraphen durch Kreise entsprechender (Kreis-)Länge repräsentiert. 
Ausgangspunkt für die Konstruktion des Minimalnetzes 6(3)3 in Abb. 3.10 sind die 1-periodischen Ketten aus Abb. 3.9.
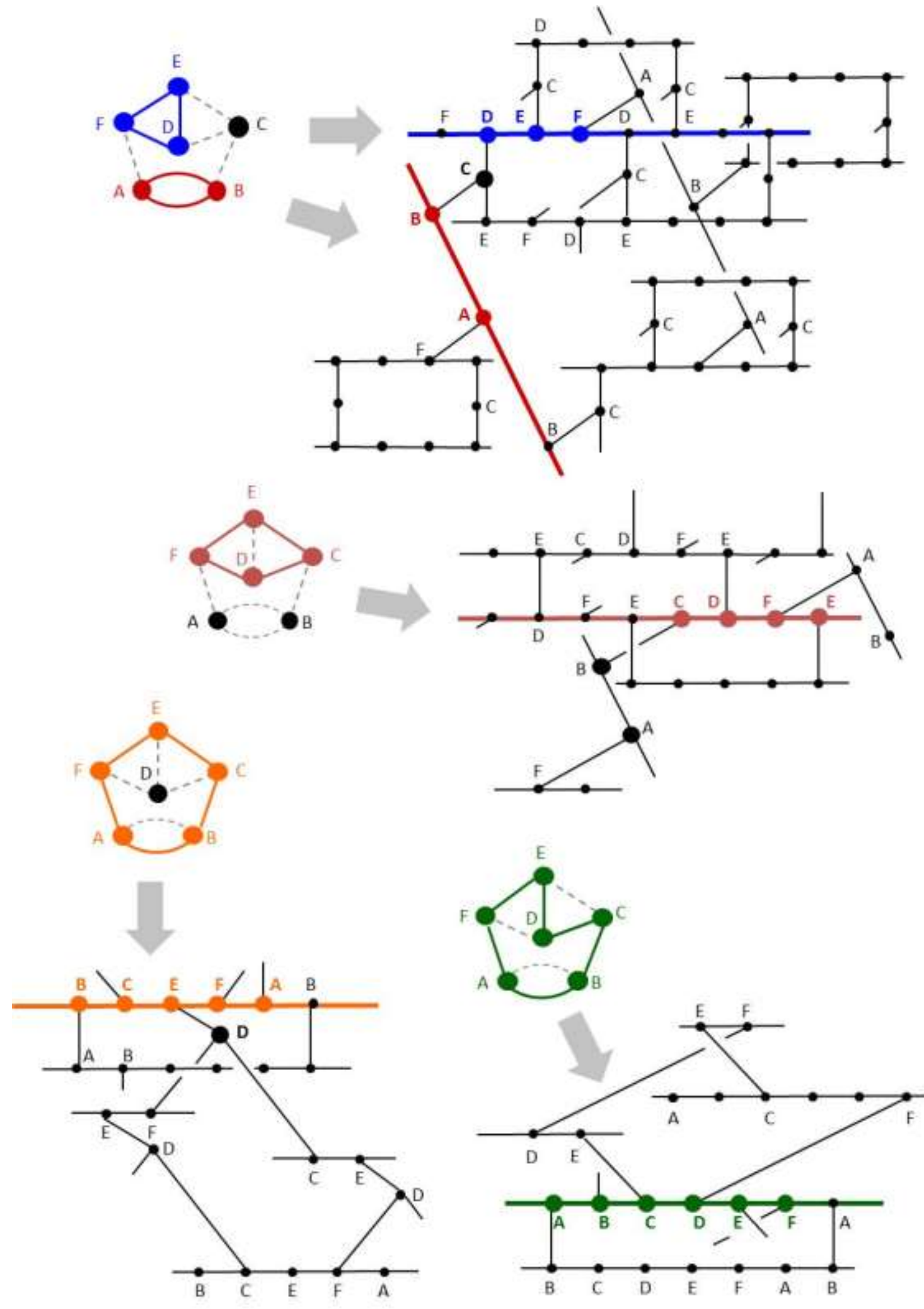

Abb. 3.10 Ketten im Netz des vierperiodischen Minimalnetzes 6(3)3. Oben eine Konstruktion des Netzes mit Ketten der Periodenlängen 2 (eine davon ist rot markiert) und 3 (eine davon ist blau markiert). Bei der Konstruktion in der Mitte ist eine Kette mit PL=4, bei der Konstruktion unten links eine Kette mit PL=5, und bei der Konstruktion unten rechts eine Kette mit PL=6 hervorgehoben. 
Die Tabelle 3.1 zeigt die Quotientengraphen der 4-periodischen Minimalnetze und mögliche Periodenlängen der 1-periodischen Ketten in deren Netzen. Die einzelnen Tabellenfelder werden auf den folgenden zwei Seiten erläutert.

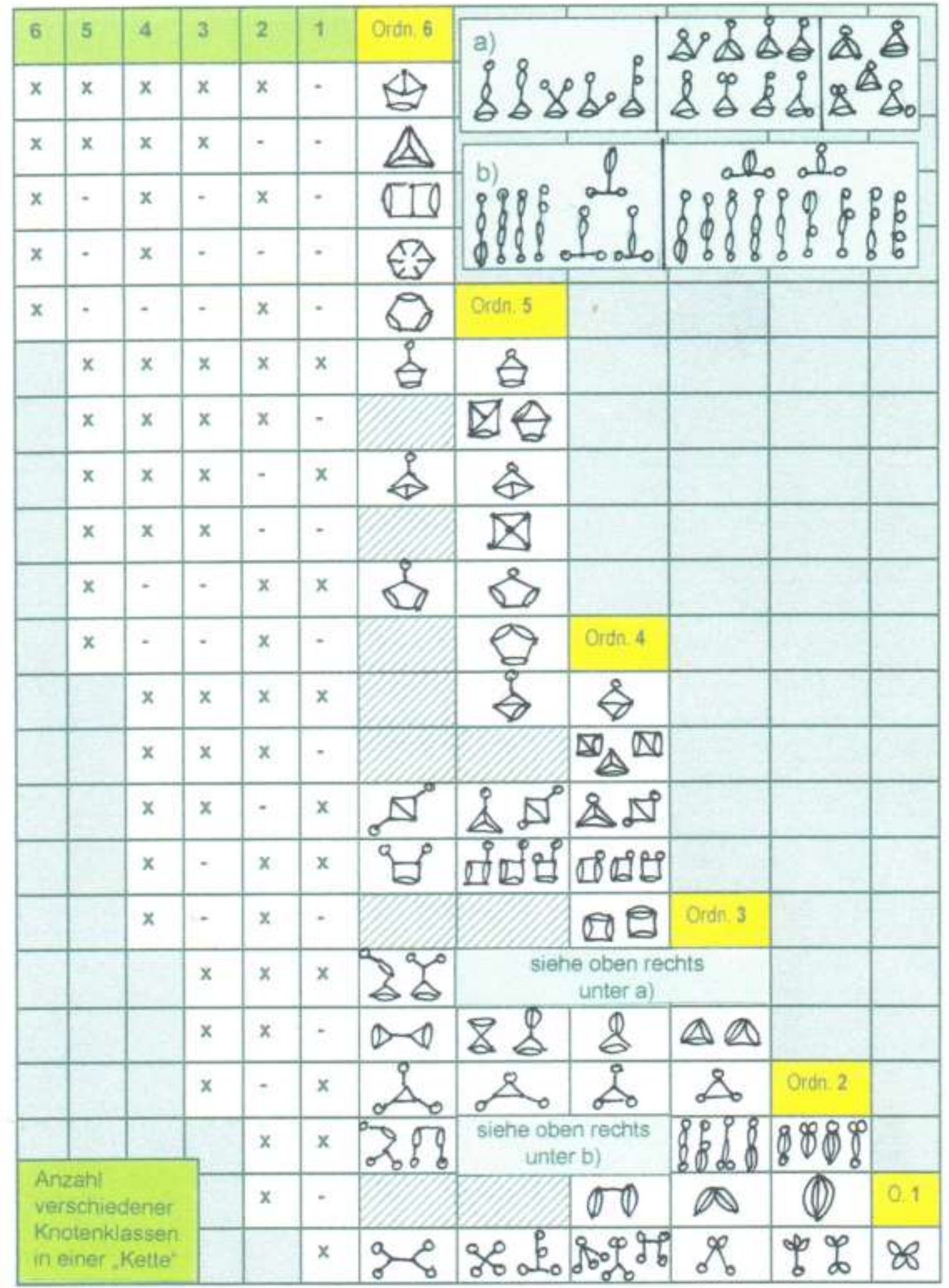

Tab. 3.1 Quotientengraphen und mögliche Periodenlängen der Ketten in deren Netzen.

Erläuterung der Felder siehe Abbildungen 3.11, 3.12, 3.13 und Text auf den folgenden zwei Seiten. 
Bedeutung der Tabellenfelder:

- $\quad$ gelbe Felder: Angabe der Ordnung des Quotientengraphen

in der dazugehörenden Spalte: die Quotientengraphen mit entsprechender Ordnung

- grüne Felder der Kopfzeile: Periodenlängen der Ketten

in der jeweils dazugehörenden Spalte:

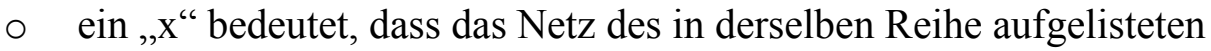
Quotientengraphen aus Ketten der entsprechenden Periodenlänge aufgebaut werden kann.

○ Ein ,-,, bedeutet, dass für dieses Netz keine Ketten der entsprechenden Periodenlänge möglich sind.

Abb. 3.11 zeigt einen Ausschnitt aus der Tabelle 3.1

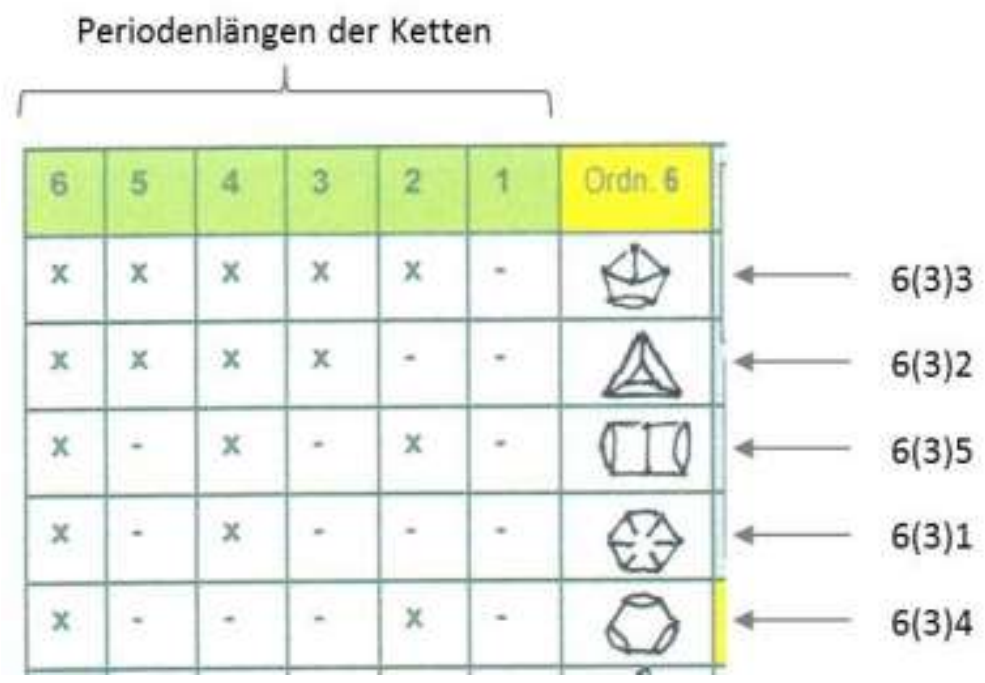

Abb. 3.11 Ausschnitt aus Tabelle 3.1 - Kopfzeile und die fünf obersten Reihen. In der ersten Reihe unterhalb der grünen Kopfzeile befindet sich der Quotientengraph des vierperiodischen Minimalnetzes 6(3)3. In der ihm zugeordneten Reihe befindet sich unter den grünen Feldern 6, 5, 4, 3 und 2 das Symbol „, ““ und unter dem grünen Feld 1 das Symbol ,-“.Das Netz 6(3)3 kann aus Ketten der Periodenlängen 2, 3, 4, 5 und 6 aufgebaut werden. In den Zeilen darunter sind die möglichen Periodenlängen von vier weiteren Netzen angegeben. 
Innerhalb einer Reihe gleichen sich die Quotientengraphen bezüglich folgender Eigenschaft: Sie können - unabhängig davon, ob sie dieselbe Ordnung haben oder nicht - mit Ketten derselben Periodenlänge aufgebaut werden.

In Abb. 3.12 ist die viertletzte Reihe der Tabelle 3.1 dargestellt. Die Minimalnetze der Quotientengraphen dieser Reihe lassen sich mit Ketten der Periodenlänge 1 oder 3 konstruieren.

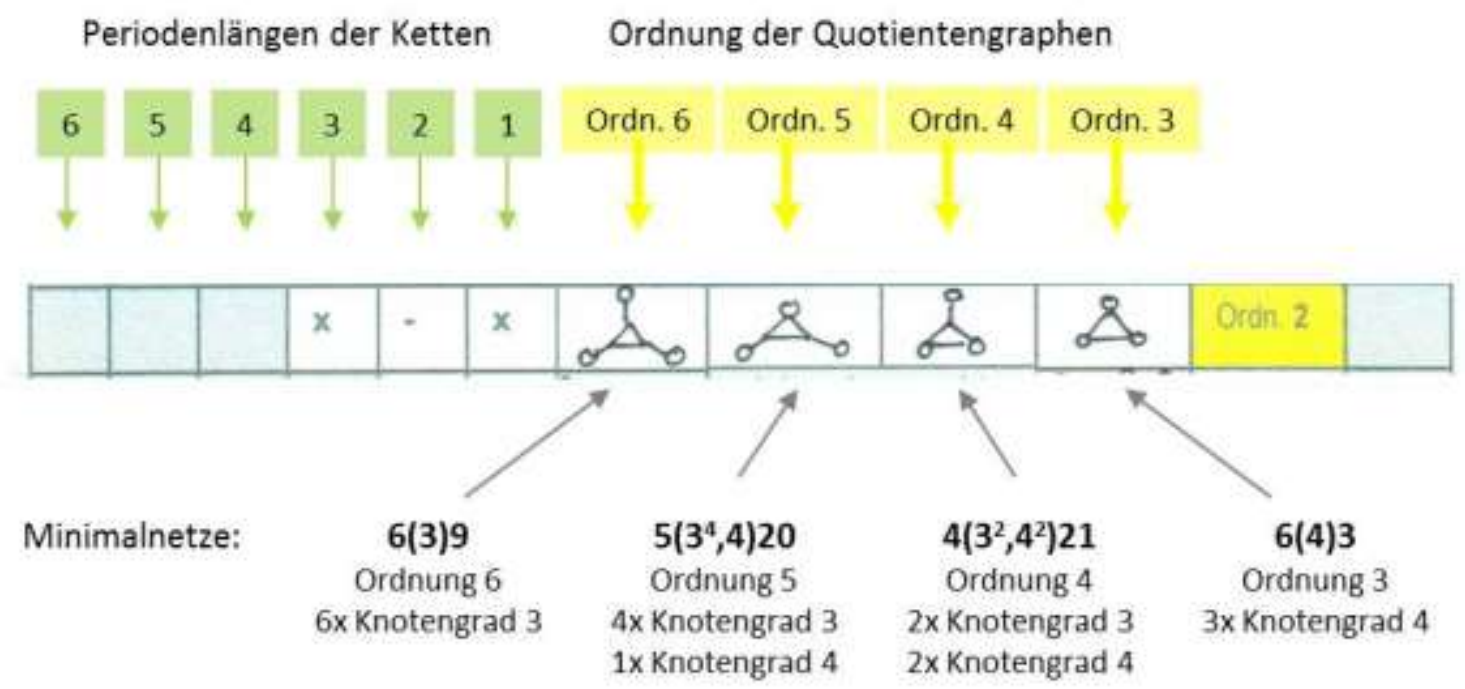

Abb. 3.12 Viertletzte Zeile der Tabelle 3.1. In dieser Zeile sind die vierperiodischen Minimalnetze mit Ketten der Periodenlängen 1 und 3 aufgeführt.

Es gibt vier Felder, zu denen sehr viele Quotientengraphen gehören. Diese Felder sind vergrößert oben rechts in der Tabelle dargestellt. Zwei der Felder sind in dieser Tabelle mit Bereich ,a)“ und zwei mit Bereich ,b)“ gekennzeichnet. In Abb. 3.13 sind die zu Bereich ,b)“ zuzuordnenden Quotientengraphen im vergrößerten Feld gezeigt.

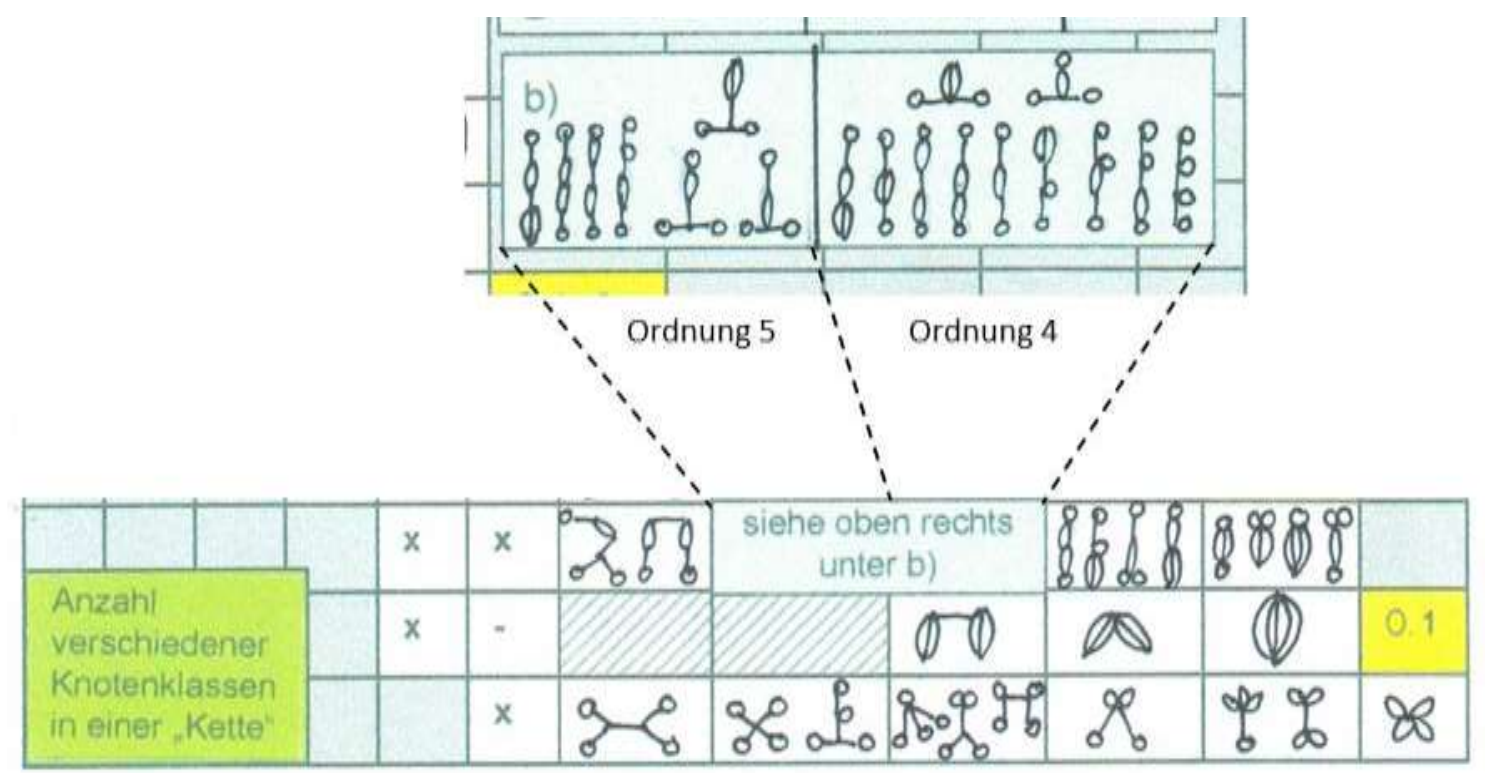

Abb. 3.13 Ausschnitt aus Tabelle 3.1 - vergrößerter Bereich „,b)“ 


\subsection{Kreise/Kreisklassen: 2-periodische Substrukturen}

Verbindet man zwei Kreise im Quotientengraphen miteinander, so ergibt dies eine Kreisklasse im periodischen Netz. Abb. 3.14 zeigt 25 einfache Teilstrukturen, die in Quotientengraphen vierperiodischer Minimalnetze vorkommen können.

Aus den drei grauhinterlegten 2-periodischen Minimalnetzen und aus Kreisen im Quotientengraphen mit 1 bis 6 Knoten lassen sich durch Hinzufügen eines Knotens (roter Pfeil, gepunktet) oder einer Kante (blauer Pfeil, gestrichelt) weitere 2-periodische Substrukturen erzeugen. Die jeweilige Länge der kürzesten Kreise dieser Substrukturen ist in der unteren Zeile angegeben.

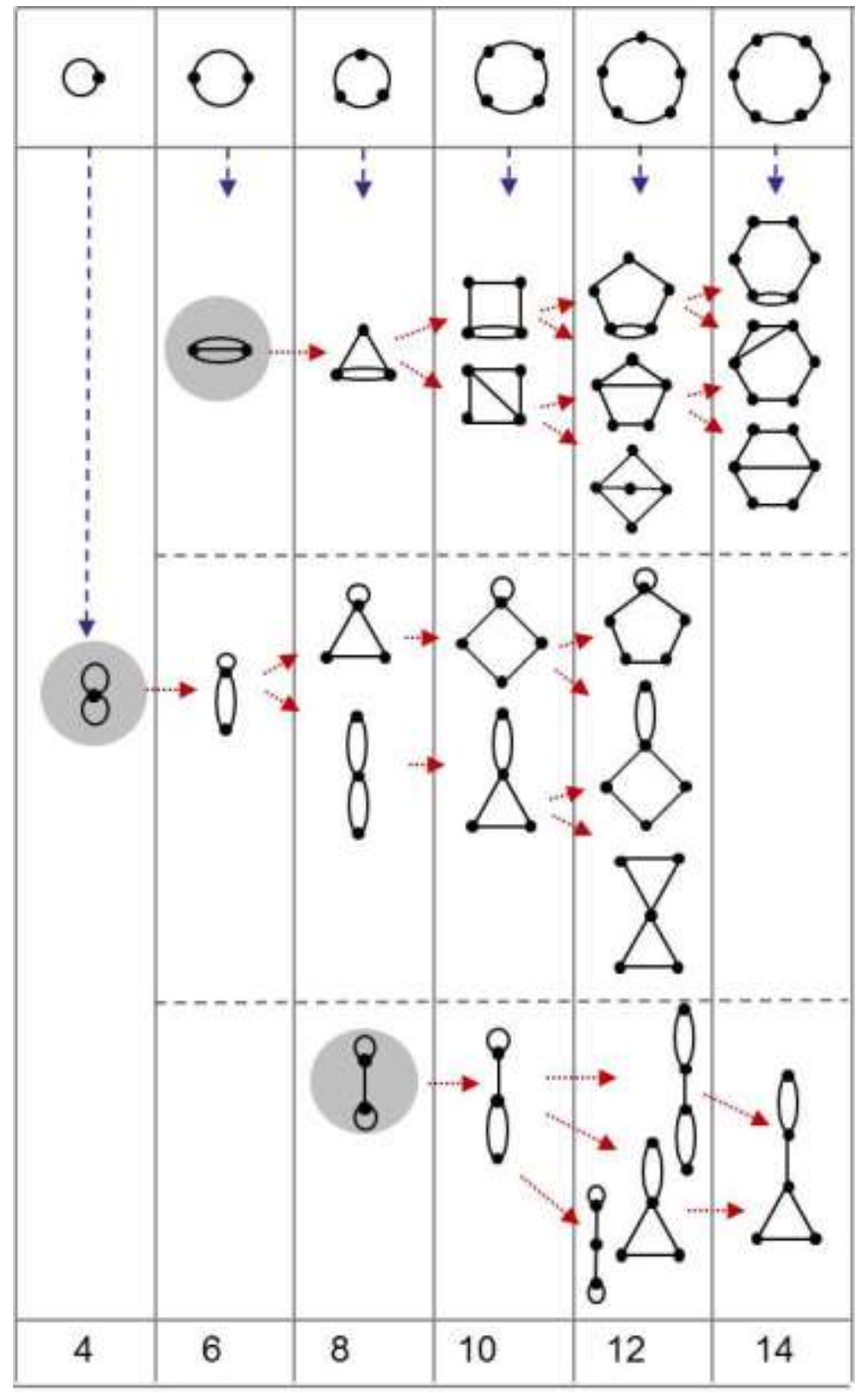

Abb. 3.14 Einfache Teilstrukturen, die in Quotientengraphen von Minimalnetzen vorkommen.. Oben: Kreise im QG mit 1 bis 6 Knoten. Gestrichelter blauer Pfeil führt zu Teilstrukturen, die durch Verbinden zweier Punkte des Kreises mit einer Kante entstehen. Grau hinterlegt: die drei 2p-Minimalnetze. Gepunkteter roter Pfeil führt zu Teilstrukturen, die durch Hinzufügen eines Knotens (o) in der Mitte einer Kante entstehen ( $\mathrm{o}-\mathrm{O} \rightarrow \mathrm{O}-\mathrm{O}-\mathrm{O}$ ). 
Aus Kreisen einer Kreisklasse lässt sich in einem n-periodischen Minimalnetz $(n \geq 3)$ eine 2-periodische Substruktur bilden.

Abb. 3.15 zeigt zwei Teilstrukturen eines Quotientengraphen und jeweils die dazugehörige 2-periodische Substruktur im Netz. Die Länge der kürzesten Kreise beträgt in beiden Fällen 8.
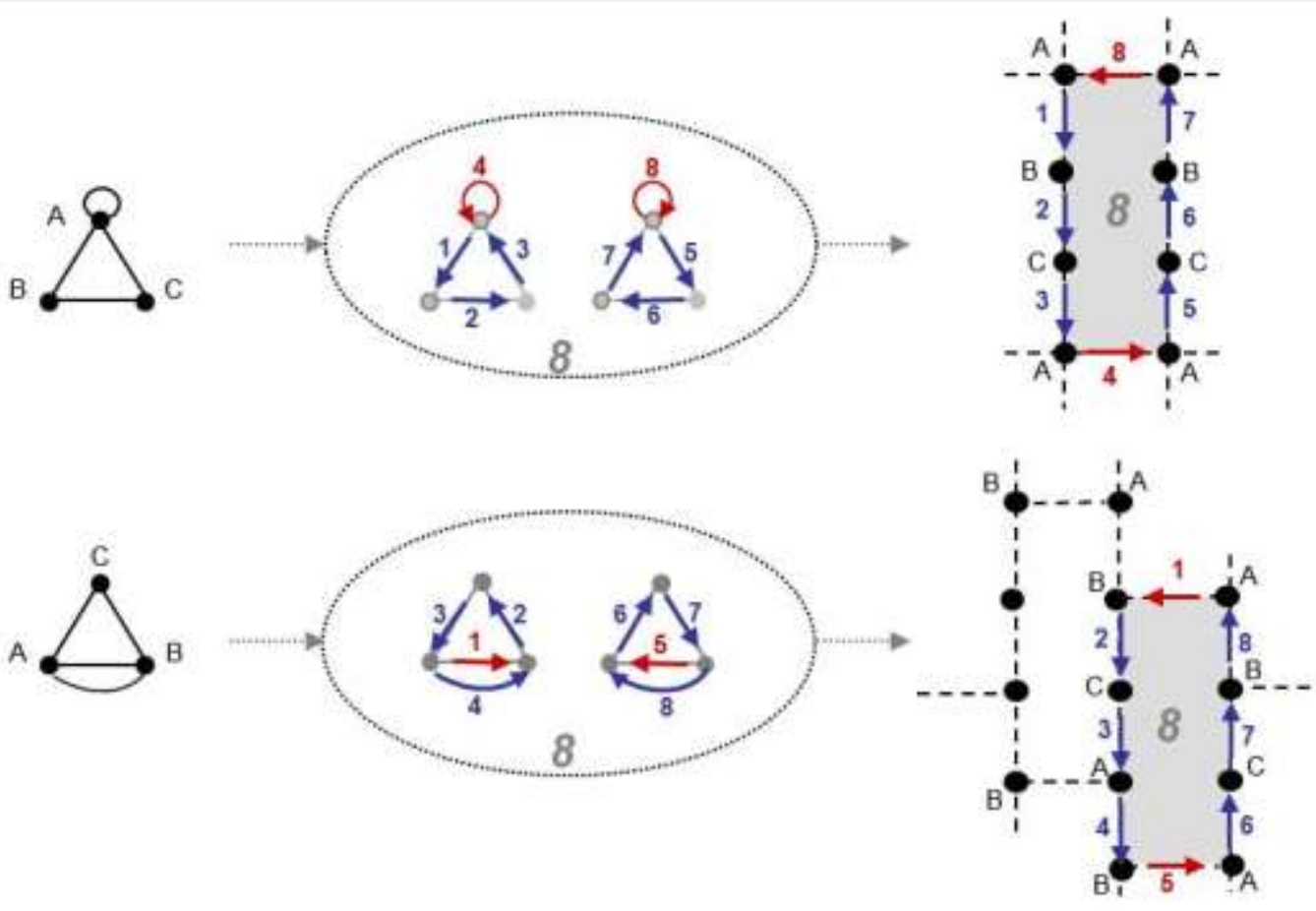

Abb. 3.15 Durchlaufen von Kreisen im Quotientengraphen. Das Bild in der Ellipse zeigt jeweils, wie ein Achterkreis (hellblaue Fläche) der 2-periodischen Substruktur (rechts) im Quotientengraphen (links) durchlaufen werden kann. Die Kanten des Kreises wurden mit 1 bis 8 durchnummeriert.

Anhand der Teilstruktur in Abb. 3.16 wird gezeigt, wieviel Kanten einer Kantenklasse zu den Kreisen der Länge 10, 12 und 14 beitragen. Bei Minimalnetzen wird zur Bildung eines Kreises von jeder Kantenklasse grundsätzlich eine geradzahlige Anzahl von Kanten benötigt.

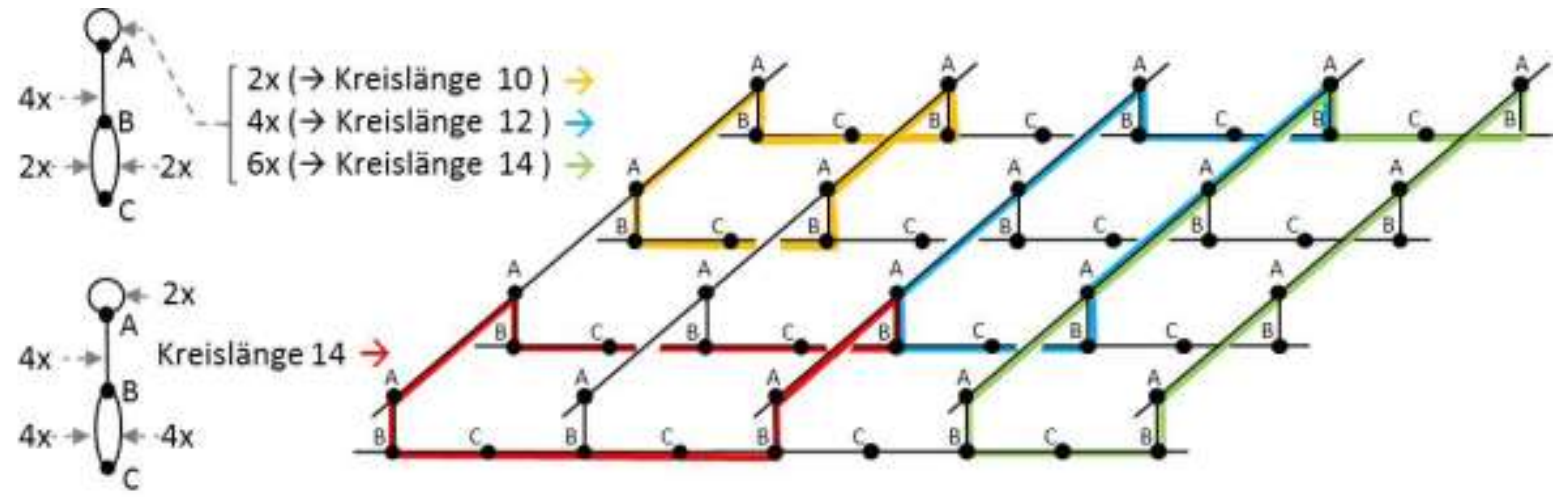

Abb. 3.16 Geradzahlige Anzahl von Kanten pro Kantenklasse in Kreisen von Minimalnetzen. Hier ist eine in manchen Minimalnetzen vorkommende Teilstruktur gezeigt. Im blauen Kreis der Länge 12 wird z. B. die Schleife an Knoten A 4x (viermal), die Kante zwischen A und B ebenfalls 4x, und jede der zwei Kanten der Doppelkante zwischen B 2x durchlaufen. 
In Abb. 3.17 werden Netzausschnitte gezeigt, die sich aus den drei 2-periodischen Minimalnetzen und aus einigen davon abgeleiteten 2-periodischen Substrukturen ergeben.

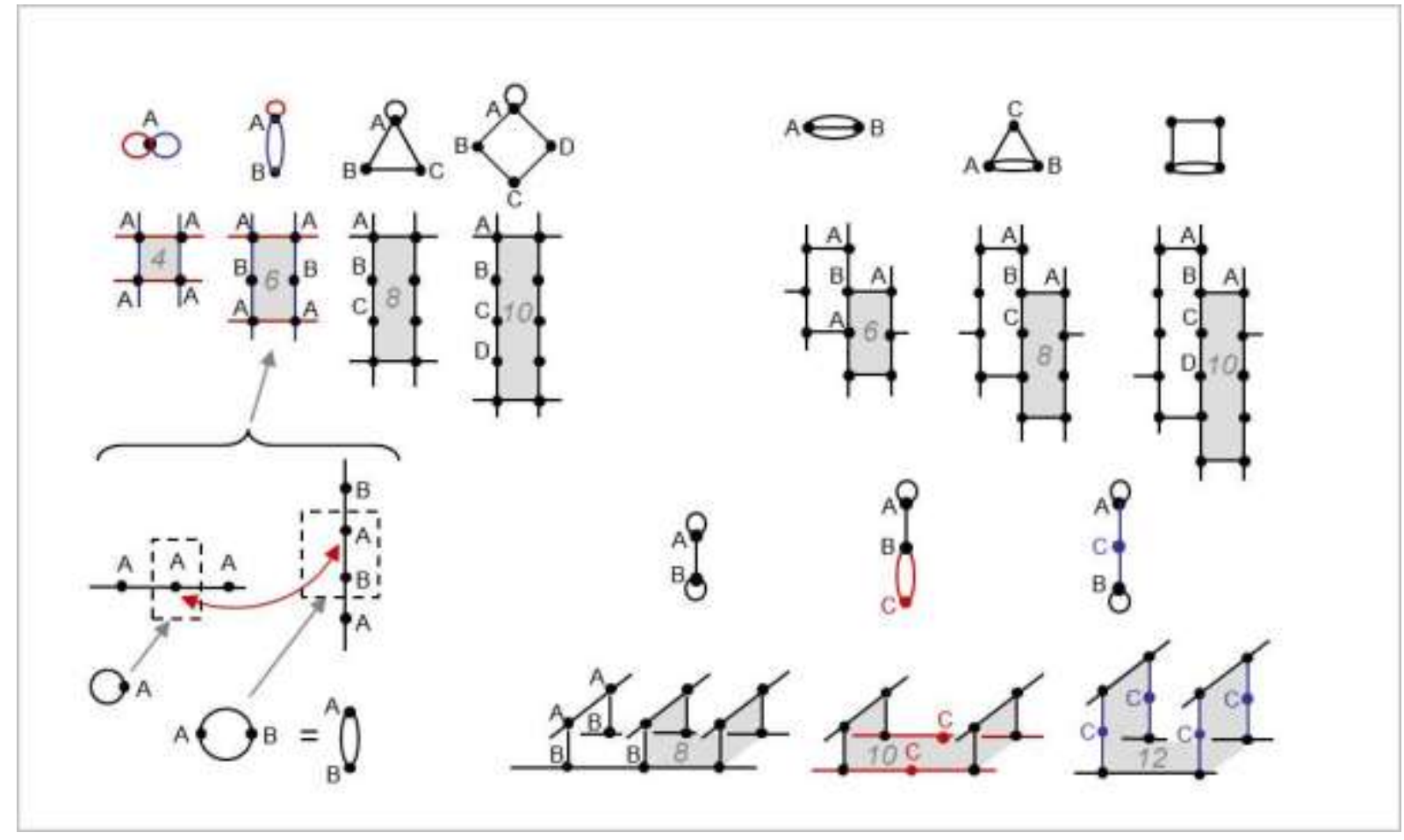

Abb. 3.17 Aus 2-periodischen Minimalnetzen abgeleitete 2-periodische Substrukturen. Unten links: das Verbinden zweier Ketten (eine mit $P L=1$, die andere mit $P L=2$ ) über ihre Knoten A führt zu einer 2-periodischen Substruktur (oben, zweite von links). Die Länge der kürzesten Kreise ist jeweils in den Flächen (hellgrau) angegeben.

In Tabelle 3.2 sind die Kreisklassen von fünf 2-periodischen Substrukturen bis zur Länge 14 aufgelistet.

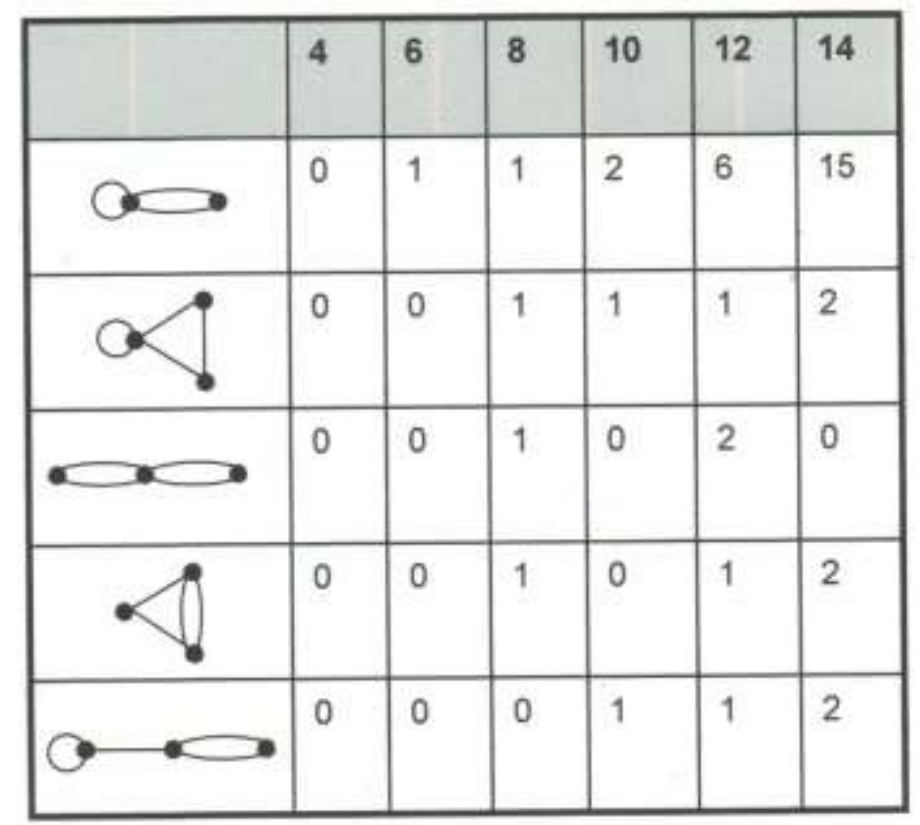

Tab. 3.2 Kreisklassenspektrum bis zur Länge 14 von fünf 2-periodischen Substrukturen 
Sind mehrere einfache Substrukturen in einem Quotientengraphen in enger Nachbarschaft, so kann die Anzahl der Kreisklassen schon bei geringer Länge stark ansteigen.

Ein gutes Beispiel hierfür ist das Minimalnetz 2(3,7)1. Bei diesem Netz sind Sechsecknetze über die eine Knotenklasse (A) mit Quadratnetzen verbunden. Das Quadratnetz wird durch a und $\mathrm{b}$ aufgespannt und ist 2-periodisch. Das Sechsecknetz hat drei gleichberechtigte Kantenklassen (c, d, e), von denen zwei für zwei weitere Periodizitäten verantwortlich sind, die dritte Kantenklasse - welche dies ist, ist abhängig von der Wahl der (topologischen) Elementarzelle - verbindet dann Punkt A mit Punkt B innerhalb der Zelle.

Um die Kreisklassen mit Kreisen der Länge 8 besser analysieren zu können, werden diese hier sieben verschiedenen Typen zugeordnet (Abb. 3.18):

- Typ 1-3: sieben Kreisklassen innerhalb des Quadratnetzes (siehe Abb. 2.11)

- Typ 4: jeweils zwei verschiedene Kanten im Sechsecknetz, dann zwei gleiche Kanten im Quadratnetz, dann der Weg zurück über Kanten dieser Kantenklassen in entgegengesetzter Richtung (Knotenfolge: A-B-A-A-A-B-A-A-)

- Typ 5 und 6 unterscheiden sich in der Position der beiden Knoten des Typs B im Kreis. Bei beiden Typen werden beide Kantenklassen des Quadratnetzes und zwei der drei Kantenklassen des Sechsecknetzes in jeweils zwei entgegengesetzte Richtungen durchlaufen.

- Typ 7: eine Kante des Quadratnetzes und drei Kanten aller drei Kantenklassen im Sechsecknetz werden in jeweils zwei entgegengesetzte Richtungen durchlaufen.

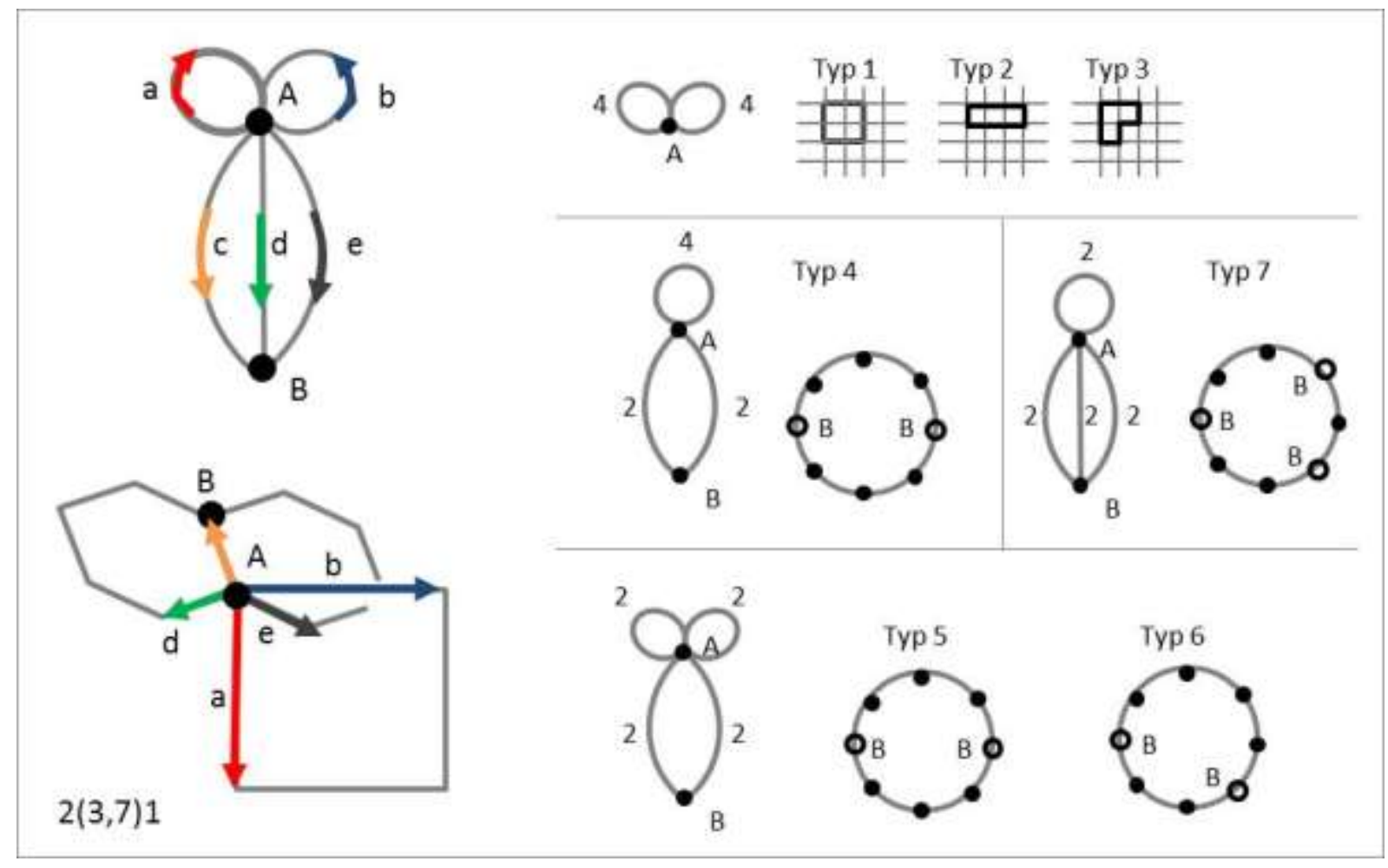

Abb. 3.18 Die sieben Typen von Kreisklassen der Länge 8 im Minimalnetz 2(3,7)1.

Quotientengraph (oben links) und Netzausschnitt (unten links). Den fünf Kantenklassen des Netzes und den Kanten des QG wurden hier die Vektoren a, b, c, d, e zugeordnet, welche dann über Linearkombination mit vier Basisvektoren zusammenhängen. Die Einteilung der Kreisklassen der Länge 8 in Typen ist oben im Text erläutert. 
Das Netz 2(3,7)1 hat 73 Kreisklassen mit Kreisen der Länge 8. Ihre unterschiedlichen Kantenfolgen sind in der folgenden zweiseitigen Tabelle (Tab.3.3) aufgelistet.

\section{$\begin{array}{llllllll}1 & 2 & 3 & 4 & 5 & 6 & 7 & 8\end{array}$}

Typ 1

Typ 2

Typ 3

Typ 4

Typ 5 $\begin{array}{llllllll}\mathbf{a} & \mathbf{a} & \mathbf{b} & \mathbf{b} & \mathbf{- a} & \mathbf{- a} & \mathbf{- b} & \mathbf{- b}\end{array}$

$\begin{array}{llllllll}\mathbf{a} & \mathbf{a} & \mathbf{a} & \mathbf{b} & \mathbf{- a} & \mathbf{- a} & \mathbf{- a} & \mathbf{- b} \\ \mathbf{a} & \mathbf{b} & \mathbf{b} & \mathbf{b} & \mathbf{- a} & \mathbf{- b} & \mathbf{- b} & \mathbf{- b}\end{array}$

$\begin{array}{llllllll}\mathbf{a} & \mathbf{a} & \mathbf{b} & \mathbf{- a} & \mathbf{b} & \mathbf{- a} & -\mathbf{b} & \mathbf{- b}\end{array}$

$\begin{array}{llllllll}\mathbf{a} & \mathbf{b} & \mathbf{a} & \mathbf{b} & \mathbf{- a} & \mathbf{- a} & \mathbf{- b} & \mathbf{- b}\end{array}$

$\begin{array}{llllllll}\mathbf{a} & \mathbf{a} & \mathbf{b} & \mathbf{b} & \mathbf{- a} & -\mathbf{b} & \mathbf{- a} & -\mathbf{b}\end{array}$

$\begin{array}{llllllll}a & b & b & -a & -a & -b & a & -b\end{array}$
8

10

11

12

13

14

15

16
4

6

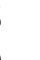

1

(n)

4

(1)

17

9

1

$$
\begin{array}{llllllll}
\text { c } & -d & \mathbf{b} & \mathbf{b} & \mathrm{d} & -\mathrm{c} & \mathbf{- b} & \mathbf{- b} \\
\mathrm{c} & -\mathrm{d} & \mathbf{a} & \mathbf{a} & \mathrm{d} & -\mathrm{c} & \mathbf{- a} & \mathbf{- a} \\
\mathrm{c} & -\mathrm{e} & \mathbf{b} & \mathbf{b} & \mathrm{e} & -\mathrm{c} & \mathbf{- b} & \mathbf{- b} \\
\mathrm{c} & -\mathrm{e} & \mathbf{a} & \mathbf{a} & \mathrm{e} & -\mathrm{c} & \mathbf{- a} & \mathbf{- a} \\
& & & & & & & \\
\mathrm{d} & -\mathrm{e} & \mathbf{b} & \mathbf{b} & \mathrm{e} & -\mathrm{d} & \mathbf{- b} & \mathbf{- b} \\
\mathrm{d} & -\mathrm{e} & \mathbf{a} & \mathbf{a} & \mathrm{e} & -\mathrm{d} & \mathbf{- a} & \mathbf{- a}
\end{array}
$$

$\begin{array}{rrrrrrrr}\text { c } & -d & \mathbf{a} & \mathbf{b} & \mathrm{d} & -\mathrm{c} & \mathbf{- a} & -\mathbf{b} \\ \mathrm{c} & -\mathrm{d} & \mathbf{a} & \mathbf{b} & \mathrm{d} & -\mathrm{c} & \mathbf{- b} & \mathbf{- a} \\ \mathrm{c} & -\mathrm{d} & \mathbf{a} & -\mathbf{b} & \mathrm{d} & -\mathrm{c} & \mathbf{- a} & \mathbf{b} \\ \mathrm{c} & -\mathrm{d} & \mathbf{a} & \mathbf{- b} & \mathrm{d} & -\mathrm{c} & \mathbf{b} & \mathbf{- a} \\ \mathrm{c} & -\mathrm{d} & -\mathbf{a} & \mathbf{b} & \mathrm{d} & -\mathrm{c} & \mathbf{a} & \mathbf{- b} \\ \mathrm{c} & -\mathrm{d} & \mathbf{- a} & \mathbf{b} & \mathrm{d} & -\mathrm{c} & \mathbf{- b} & \mathbf{a} \\ \mathrm{c} & -\mathrm{d} & -\mathbf{a} & -\mathbf{b} & \mathrm{d} & -\mathrm{c} & \mathbf{a} & \mathbf{b} \\ \mathrm{c} & -\mathrm{d} & -\mathbf{a} & -\mathbf{b} & \mathrm{d} & -\mathrm{c} & \mathbf{b} & \mathbf{a}\end{array}$

$\begin{array}{llllllll}c & -\mathrm{e} & \mathbf{a} & \mathbf{b} & \mathrm{e} & -\mathrm{c} & \mathbf{- a} & \mathbf{- b}\end{array}$

$\begin{array}{llllllll}c & -\mathrm{e} & \mathbf{a} & \mathbf{b} & \mathrm{e} & -\mathrm{c} & \mathbf{- b} & \mathbf{- a}\end{array}$

$\begin{array}{llllllll}c & -\mathrm{e} & \mathbf{a} & \mathbf{- b} & \mathrm{e} & -\mathrm{c} & \mathbf{- a} & \mathbf{b}\end{array}$

$\begin{array}{llllllll}c & -\mathrm{e} & \mathbf{a} & \mathbf{- b} & \mathrm{e} & -\mathrm{c} & \mathbf{b} & \mathbf{- a}\end{array}$

$\begin{array}{llllllll}\text { c } & -\mathrm{e} & \mathbf{- a} & \mathbf{b} & \mathrm{e} & -\mathrm{c} & \mathbf{a} & \mathbf{- b}\end{array}$

$\begin{array}{llllllll}c & -\mathrm{e} & -\mathbf{a} & \mathbf{b} & \mathrm{e} & -\mathrm{c} & \mathbf{- b} & \mathbf{a}\end{array}$

$\begin{array}{llllllll}\text { c } & -\mathrm{e} & -\mathbf{a} & \mathbf{- b} & \mathrm{e} & -\mathrm{c} & \mathbf{a} & \mathbf{b}\end{array}$

$\begin{array}{llllllll}c & -\mathrm{e} & -\mathbf{a} & -\mathbf{b} & \mathrm{e} & -\mathrm{c} & \mathbf{b} & \mathbf{a}\end{array}$

$\begin{array}{llllllll}\mathrm{d} & -\mathrm{e} & \mathbf{a} & \mathbf{b} & \mathrm{e} & -\mathrm{d} & \mathbf{- a} & \mathbf{- b}\end{array}$

$\begin{array}{llllllll}\mathrm{d} & -\mathrm{e} & \mathbf{a} & \mathbf{- b} & \mathrm{e} & -\mathrm{d} & \mathbf{- a} & \mathbf{b}\end{array}$

$\begin{array}{llllllll}\mathrm{d} & -\mathrm{e} & \mathbf{a} & \mathbf{- b} & \mathrm{e} & -\mathrm{d} & \mathbf{b} & \mathbf{- a}\end{array}$

$\begin{array}{llllllll}\mathrm{d} & -\mathrm{e} & -\mathbf{a} & \mathbf{b} & \mathrm{e} & -\mathrm{d} & \mathbf{a} & -\mathbf{b}\end{array}$

$\begin{array}{llllllll}\mathrm{d} & -\mathrm{e} & -\mathbf{a} & \mathbf{b} & \mathrm{e} & -\mathrm{d} & \mathbf{- b} & \mathbf{a}\end{array}$

$\begin{array}{llllllll}\mathrm{d} & -\mathrm{e} & -\mathbf{a} & -\mathbf{b} & \mathrm{e} & -\mathrm{d} & \mathbf{a} & \mathbf{b}\end{array}$

$\begin{array}{llllllll}\mathrm{d} & -\mathrm{e} & -\mathbf{a} & -\mathbf{b} & \mathrm{e} & -\mathrm{d} & \mathbf{b} & \mathbf{a}\end{array}$ $\begin{array}{llllllll}\mathrm{d} & -\mathrm{e} & \mathbf{a} & \mathbf{b} & \mathrm{e} & -\mathrm{d} & \mathbf{- b} & \mathbf{- a}\end{array}$ 
Typ 6

\begin{tabular}{|c|c|c|c|c|c|c|c|c|c|}
\hline & 38 & $\mathrm{c}$ & $-d$ & $\mathbf{a}$ & $\mathrm{d}$ & $-c$ & b & $-\mathbf{a}$ & $-\mathbf{b}$ \\
\hline & 39 & $\mathrm{c}$ & $-d$ & $\mathbf{a}$ & d & $-c$ & $-b$ & $-\mathbf{a}$ & b \\
\hline & 40 & c & $-d$ & b & $\mathrm{d}$ & $-c$ & $\mathbf{a}$ & $-b$ & $-\mathbf{a}$ \\
\hline & 41 & $\mathrm{c}$ & $-d$ & b & $\mathrm{d}$ & $-c$ & $-\mathbf{a}$ & $-b$ & $-\mathbf{a}$ \\
\hline & 42 & $\mathrm{c}$ & $-d$ & b & $\mathbf{a}$ & $-b$ & $\mathrm{~d}$ & $-c$ & $-\mathbf{a}$ \\
\hline & 43 & $\mathrm{c}$ & $-d$ & $\mathbf{a}$ & b & $-\mathbf{a}$ & d & $-c$ & $-b$ \\
\hline & 44 & $\mathrm{c}$ & $-d$ & $-\mathbf{b}$ & $\mathbf{a}$ & b & $\mathrm{d}$ & $-c$ & $-\mathbf{a}$ \\
\hline & 45 & $c$ & $-d$ & $-\mathbf{a}$ & b & $\mathbf{a}$ & d & $-c$ & $-b$ \\
\hline & 46 & $\mathrm{c}$ & $-e$ & $\mathbf{a}$ & $\mathrm{e}$ & $-c$ & b & $-\mathbf{a}$ & $-b$ \\
\hline & 47 & $\mathrm{c}$ & $-e$ & $\mathbf{a}$ & $\mathrm{e}$ & $-c$ & $-b$ & $-\mathbf{a}$ & b \\
\hline & 48 & $\mathrm{c}$ & $-e$ & b & $\mathrm{e}$ & $-c$ & $\mathbf{a}$ & $-b$ & $-\mathbf{a}$ \\
\hline & 49 & $\mathrm{c}$ & $-e$ & b & $\mathrm{e}$ & $-c$ & $-\mathbf{a}$ & $-b$ & $-\mathbf{a}$ \\
\hline & 50 & $\mathrm{c}$ & $-e$ & b & $\mathbf{a}$ & $-b$ & $\mathrm{e}$ & $-c$ & $-\mathbf{a}$ \\
\hline & 51 & $\mathrm{c}$ & $-e$ & $\mathbf{a}$ & b & $-\mathbf{a}$ & e & $-c$ & $-b$ \\
\hline & 52 & $\mathrm{c}$ & $-e$ & $-b$ & $\mathbf{a}$ & $\mathbf{b}$ & $\mathrm{e}$ & $-c$ & $-\mathbf{a}$ \\
\hline & 53 & $\mathrm{c}$ & $-e$ & $-\mathbf{a}$ & b & $\mathbf{a}$ & $\mathrm{e}$ & $-c$ & $-b$ \\
\hline & 54 & d & $-e$ & $\mathbf{a}$ & $\mathrm{e}$ & $-d$ & b & $-\mathbf{a}$ & $-b$ \\
\hline & 55 & d & $-e$ & $\mathbf{a}$ & $\mathrm{e}$ & $-d$ & $-b$ & $-\mathbf{a}$ & b \\
\hline & 56 & d & $-e$ & b & $\mathrm{e}$ & $-d$ & $\mathbf{a}$ & $-b$ & $\mathbf{- a}$ \\
\hline & 57 & d & $-e$ & b & $\mathrm{e}$ & $-d$ & $-\mathbf{a}$ & $-b$ & $\mathbf{- a}$ \\
\hline & 58 & d & $-e$ & b & $\mathbf{a}$ & $-b$ & $\mathrm{e}$ & $-d$ & $\mathbf{- a}$ \\
\hline & 59 & d & $-e$ & $\mathbf{a}$ & b & $-\mathbf{a}$ & e & $-d$ & $-b$ \\
\hline & 60 & d & $-e$ & $-\mathbf{b}$ & $\mathbf{a}$ & b & e & $-d$ & $\mathbf{- a}$ \\
\hline & 61 & d & $-e$ & $-\mathbf{a}$ & b & $\mathbf{a}$ & $\mathrm{e}$ & $-d$ & $-b$ \\
\hline \multicolumn{10}{|l|}{ Тур 7} \\
\hline & 62 & $\mathrm{c}$ & $-d$ & $\mathbf{a}$ & e & $-c$ & d & $-\mathrm{e}$ & $\mathbf{- a}$ \\
\hline & 63 & $\mathrm{c}$ & $-d$ & $-\mathbf{a}$ & e & $-c$ & d & $-\mathrm{e}$ & $\mathbf{a}$ \\
\hline & 64 & $\mathrm{c}$ & $-d$ & b & $\mathrm{e}$ & $-c$ & d & $-e$ & $-b$ \\
\hline & 65 & $\mathrm{c}$ & $-d$ & $-b$ & $\mathrm{e}$ & $-c$ & d & $-\mathrm{e}$ & $\mathbf{b}$ \\
\hline & 66 & $\mathrm{c}$ & $-\mathrm{e}$ & $\mathbf{a}$ & $\mathrm{d}$ & $-c$ & $\mathrm{e}$ & $-d$ & $\mathbf{- a}$ \\
\hline & 67 & $\mathrm{c}$ & $-e$ & $\mathbf{- a}$ & $\mathrm{d}$ & $-c$ & $\mathrm{e}$ & $-d$ & $\mathbf{a}$ \\
\hline & 68 & $\mathrm{c}$ & $-e$ & $\mathbf{b}$ & $\mathrm{d}$ & $-c$ & $\mathrm{e}$ & $-d$ & $-b$ \\
\hline & 69 & $\mathrm{c}$ & $-e$ & $-\mathbf{b}$ & $\mathrm{d}$ & $-c$ & $\mathrm{e}$ & $-d$ & $\mathbf{b}$ \\
\hline & 70 & d & $-e$ & $\mathbf{a}$ & $\mathrm{e}$ & $-d$ & $\mathrm{e}$ & $-c$ & $\mathbf{- a}$ \\
\hline & 71 & $\mathrm{~d}$ & $-e$ & $\mathbf{- a}$ & $\mathrm{e}$ & $-d$ & $\mathrm{e}$ & $-c$ & $\mathbf{a}$ \\
\hline & 72 & $\mathrm{~d}$ & $-e$ & $\mathbf{b}$ & $\mathrm{e}$ & $-d$ & $\mathrm{e}$ & $-c$ & -b \\
\hline & 73 & $\mathrm{~d}$ & $-\mathrm{e}$ & $-b$ & $\mathrm{e}$ & $-\mathrm{d}$ & $\mathrm{e}$ & $-c$ & $\mathbf{b}$ \\
\hline
\end{tabular}

Tab. 3.3 Die 73 Kreisklassen mit Kreisen der Länge 8 des Minimalnetzes 2(3,7)1.

Erläuterung zu Typen und Kantenbezeichnungen siehe Abb. 3.18 und Textabschnitt davor. 
In Abb. 3.19 sind in der oberen Bildhälfte ein 10er- und ein 14er-Kreis des Minimalnetzes 6(3)5, und in der unteren Bildhälfte ein 12er- und ein 14er-Kreis des Minimalnetzes 6(3)4 dargestellt. Der 14er-Kreis, der durch blaue Kanten dargestellt ist, ist jeweils isomorph zum 14er-Kreis, der durch die graue Fläche gekennzeichnet ist. Die beiden Darstellungen der 14er-Kreise mit den blauen Flächen zeigen, dass in beiden Netze eine topologisch identische 2-periodische Substruktur aus solchen 14er-Kreisen aufgebaut werden kann.
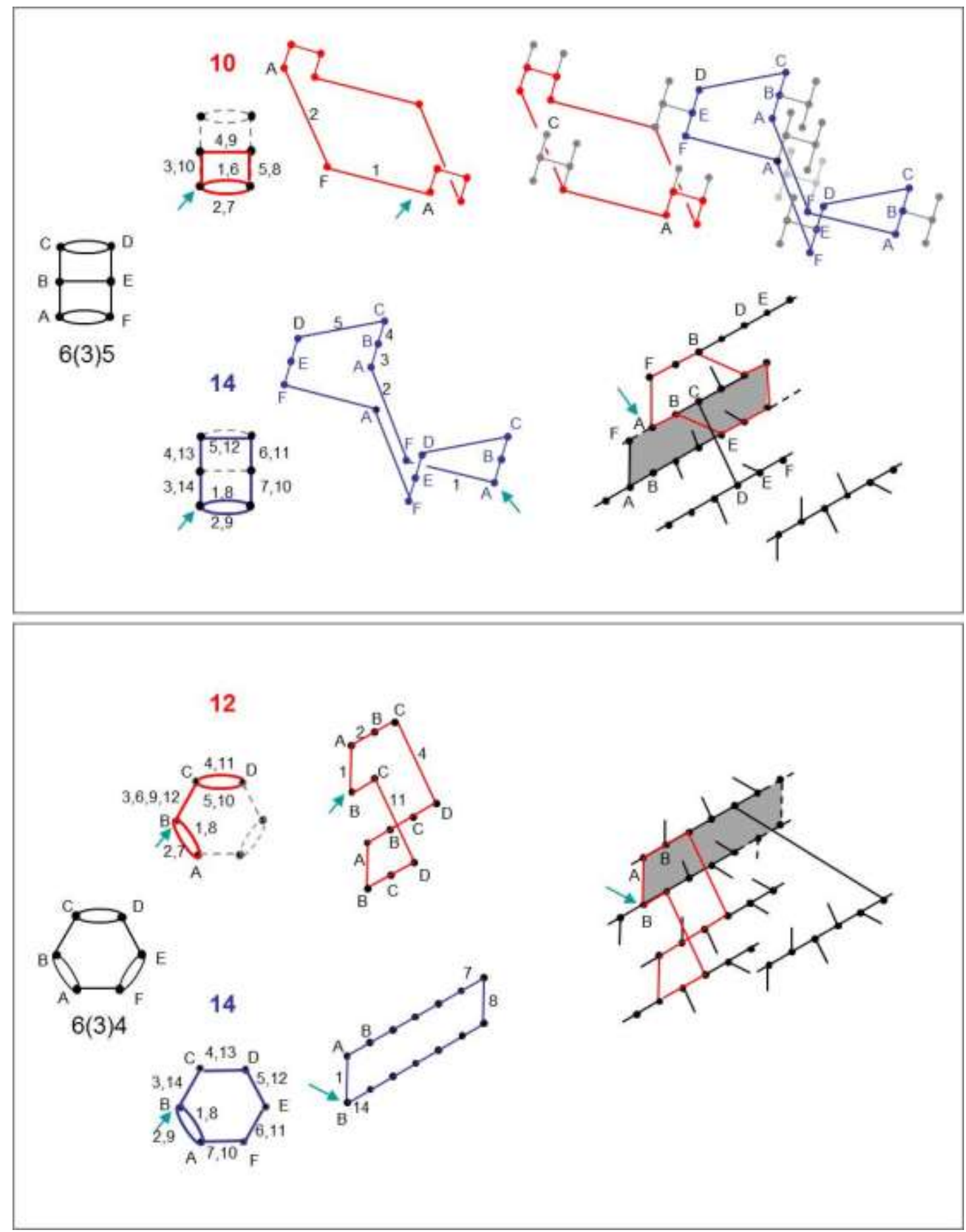

Abb. 3.19 Topologisch identische 2-periodische Substrukturen. Rot: 10er-Kreis von 6(3)5 bzw. 12er-Kreis von 6(3)4. Blau und graue Fläche: 14er-Kreise. Aus direkt zusammenhängenden 14er-Kreisen dieser Kreisklasse entsteht eine 2p-Substruktur, die in beiden Netzen topologisch identisch ist. Der Pfeil zeigt jeweils auf den Knoten, der in Bezug auf die hier vorgenommene Durchnummerierung der Kanten eines Kreises vor Kante 1 liegt. 


\subsection{Netze: 2- und 3-periodische Minimalnetze}

Ist ein Teilgraph eines Quotientengraphen eines Minimalnetzes (Nr. 1) ein Quotientengraph eines anderen Minimalnetzes (Nr. 2), so ist das Minimalnetz Nr. 2 ein Subnetz des Minimalnetzes Nr. 1. Das Netz Nr. 2 hat geringere Periodizität als das Netz Nr. 1.

In vierperiodischen Minimalnetzen der Ordnung 6 haben alle Knoten den Knotengrad 3, und das Löschen einer beliebigen Kante lässt Knoten mit einem Knotengrad < 3 zurück. Es lassen sich hier keine Minimalnetze geringerer Periodizität als Subnetz finden. In vielen vierperiodischen Minimalnetzen geringerer Ordnung als 6 gibt es Subnetze, die Minimalnetze geringerer Periodizität sind.

Abb. 3.20 erläutert anhand von Beispielen die in Abb. 3.21 dargestellten Subnetzbeziehungen:

- Beim Minimalnetz 2(3,7)1 (Abb. 3.18) lassen sich sowohl das 3-periodische Minimalnetz 2(3,5)1 als auch die zwei 2-periodischen Minimalnetze 1(4)1 und 2(3)1 (Abb. 2.13) als Subnetze finden.

- Die Minimalnetze 1(6)1 und 1(4)1 (Abb. 2.5 rechts unten und rechts oben) sind Subnetze des Minimalnetzes 1(8)1 (Abb. 3.2).

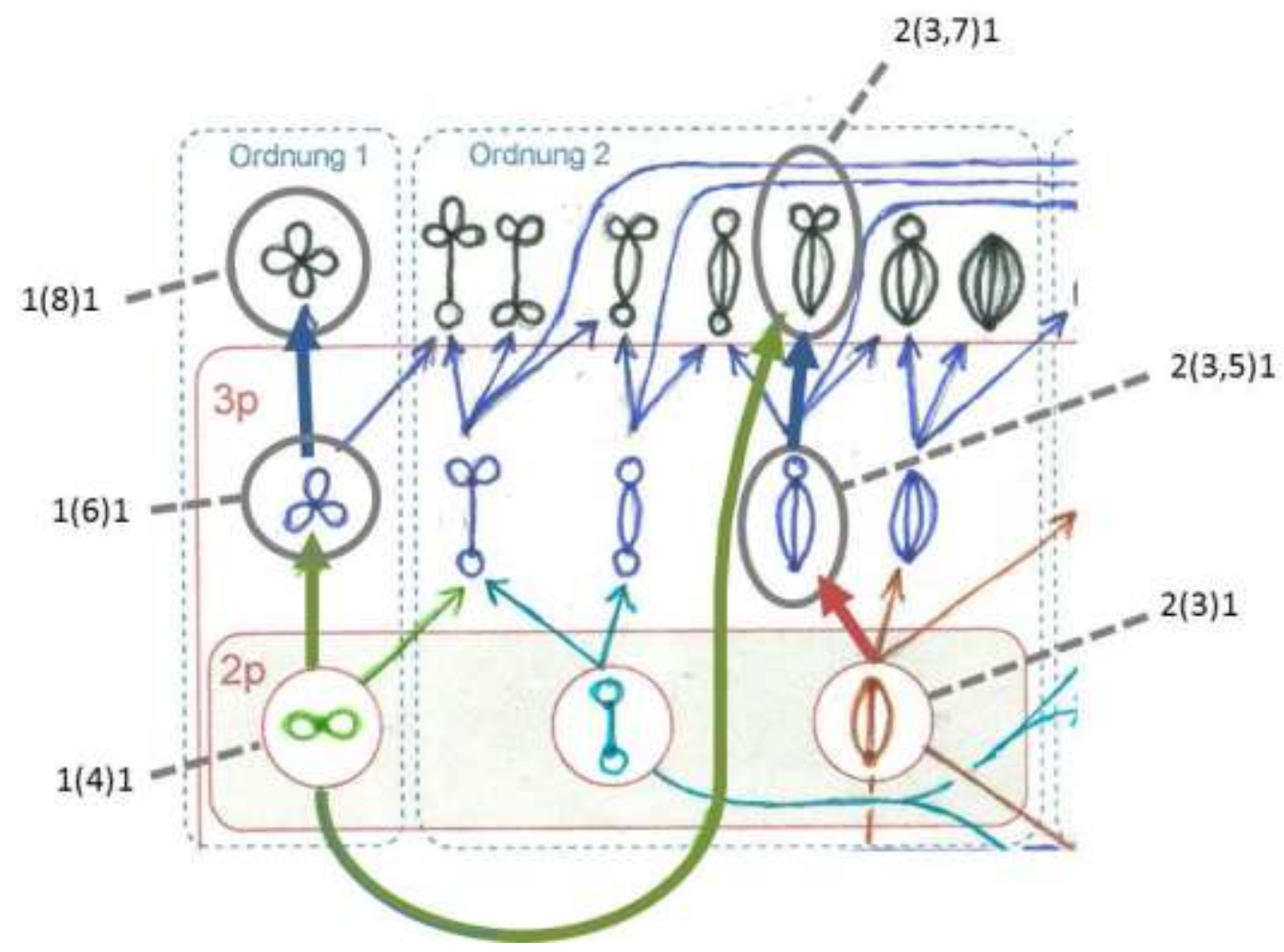

Abb. 3.20 Minimalnetze als Substrukturen der Minimalnetze 2(3,7)1 und 1(8)1. Diese Abbildung zeigt einen Ausschnitt aus Abb. 3.21. Die Pfeile zeigen jeweils in Richtung steigender Periodizität. Die Subnetzbeziehungen der obigen Beispiele sind durch die fett gezeichneten Pfeile gekennzeichnet. Das Minimalnetz 1(4)1 ist Subnetz der Minimalnetze 1(6)1, 1(8)1 und 2(3,7)1. In 2(3,7)1 ist 2(3,5)1 als Subnetz enthalten, und beide haben das Minimalnetz 2(3)1 als Subnetz.

Die Subnetzbeziehungen zwischen 2-, 3- und 4-periodischen Minimalnetzen sind in Abb. 3.21 auf der folgenden Seite dargestellt. 


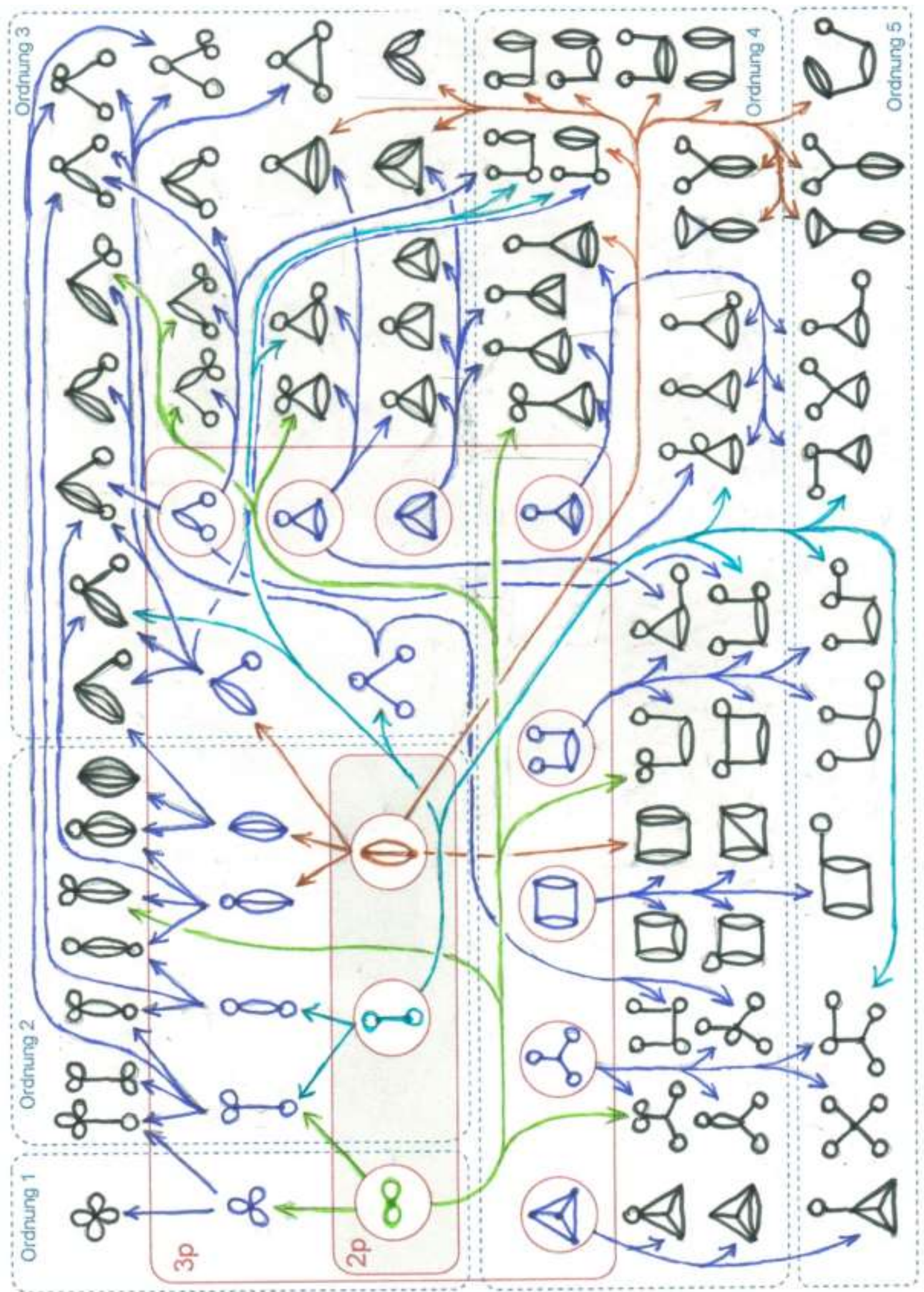

Abb. 3.21 Subnetzbeziehungen zwischen 2-, 3- und 4-periodischen Minimalnetzen.

Von den drei zweiperiodischen Minimalnetzen (2p) führen Pfeile zu den höherperiodischen Minimalnetzen, die die jeweiligen Netze als Subnetz haben: entweder direkt oder über ein dazwischenliegendes Netz. Verfolgt man die Pfade entgegen der Pfeilrichtung, lassen sich zu einem Minimalnetz all seine Subnetze, die Minimalnetze geringer Periodizität sind, finden. 
In Abb. 3.22 sind mehrere Substrukturen des Minimalnetzes $4\left(3^{3}, 5\right) 10$ gezeigt:

- orange: eine Substruktur, die kein Netz ist

- rot: das 2-periodische Minimalnetz 2(3)2, kürzeste Kreise sind 8er-Kreise

- blau: das 3-periodische Minimalnetz 4(3)5, kürzeste Kreise sind 10er-Kreise

- türkis: das 3-periodische Minimalnetz $3\left(3^{2}, 4\right) 5$, kürzeste Kreise sind 6er-Kreise

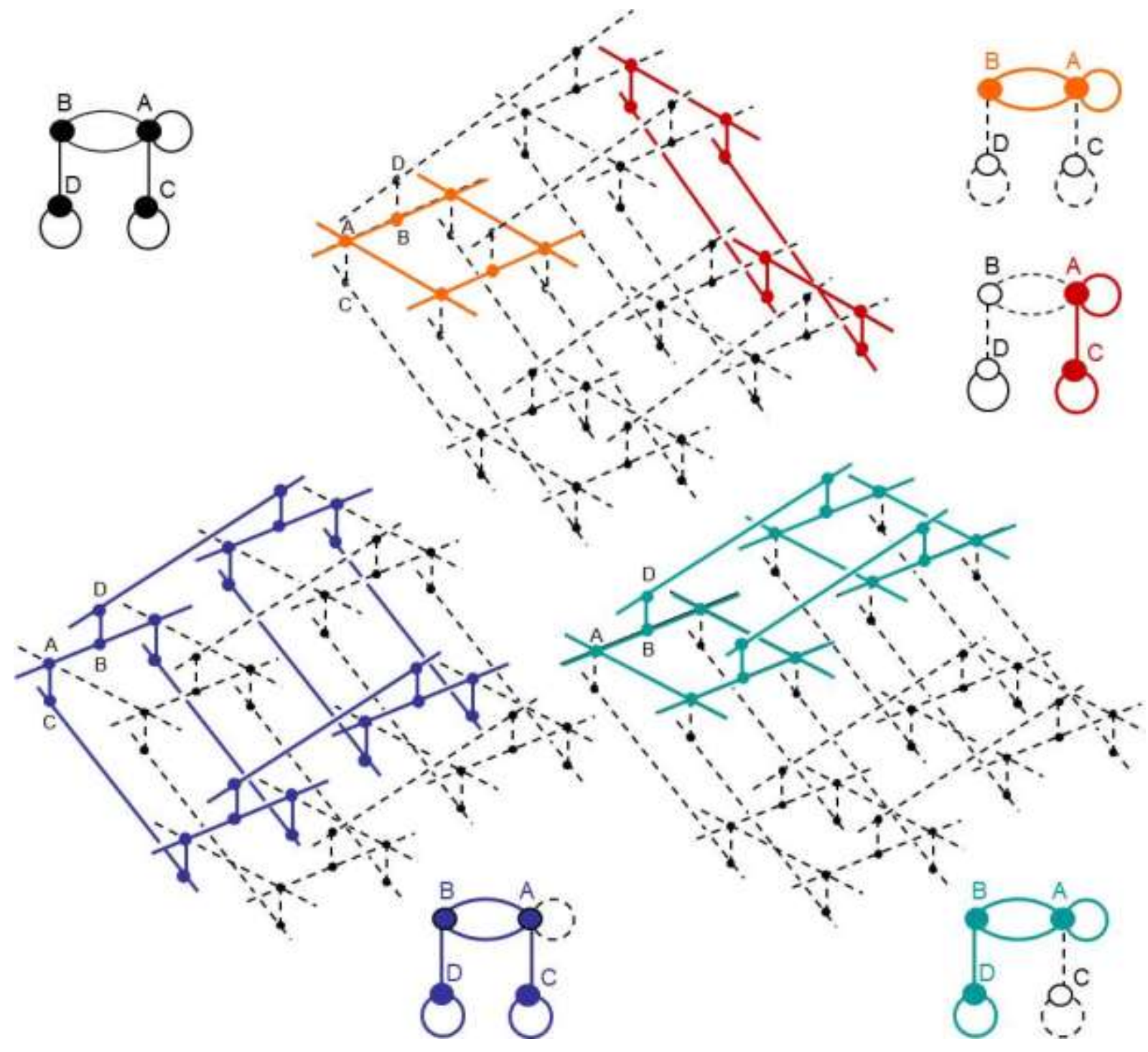

Abb. 3.22 Substrukturen des Minimalnetzes 4(3 $\left.3^{3}, 5\right) 10$. Drei Minimalnetze geringerer Periodizität sind Substrukturen dieses vierperiodischen Minimalnetzes: Das zweiperiodische Minimalnetz 2(3)2 (rot), sowie die zwei dreiperiodischen Minimalnetze 4(3)5 (blau) und 3(32 4$) 5$ (türkis).

Im Minimalnetz $4\left(3^{3}, 5\right) 10$ sind Kreise der Längen enthalten, die in seinen Subnetzen auch schon vorhanden sind. Es muss also Kreisklassen mit Kreisen der Längen 6, 8 und 10 haben:

\begin{tabular}{lcccc} 
Kreislänge & 4 & 6 & 8 & 10 \\
\hdashline Kreisklassen & 0 & 1 & 2 & 6
\end{tabular}




\section{Erzeugen von 4p-Minimalnetzen aus 4p-Minimalnetzen anderer Ordnung}

Um bestimmte topologische Beziehungen zwischen 3-dimensionalen uninodalen Netzen zu untersuchen, stellt V. A. Blatov eine Methode vor, die durch Reduzierung des Knotengrades aus einem Ausgangsnetz (Supernet) uninodale Subnetze erzeugt [Blatov, 2007]. Im Folgenden wird gezeigt, wie vierperiodische Minimalnetze, deren Quotientengraphen eine bestimmte Ordnung (Anzahl der Knoten) haben, aus vierperiodischen Minimalnetzen mit Quotientengraphen einer anderen Ordnung gebildet werden können.

Es sind dabei zwei Vorgänge zu unterscheiden:

- Erhöhung der Ordnung um $1 \quad(1 \rightarrow 2 \ldots \rightarrow 6)$

- Verringerung der Ordnung um $1 \quad(6 \rightarrow 5 \ldots \rightarrow 1)$

Jeder dieser beiden Vorgänge kann mehrmals hintereinander ausgeführt werden. Diese beiden Vorgänge können miteinander kombiniert werden (z. B. $3 \rightarrow 4 \rightarrow 3$ ).

\subsection{Erhöhung der Ordnung}

Wird ein Knoten im Quotientengraphen durch den Graphen $\mathrm{K}_{2}$ ersetzt, und werden dabei die vormals am Knoten liegenden Kantenenden auf die beiden Knoten des $\mathrm{K}_{2}$ verteilt, so entsteht ein Quotientengraph, dessen Ordnung um 1 höher ist. Werden alle Möglichkeiten, die Enden der Kanten dann auf die beiden Knoten zu verteilen, ausgeschöpft, so können bei wiederholter Prozedur aus dem vierperiodischen Gitternetz systematisch alle Minimalnetze erzeugt werden.

Nach der Aktion, kann

- eine Schleife mit beiden Enden mit einem der beiden Knoten des $\mathrm{K}_{2}$ verbunden sein

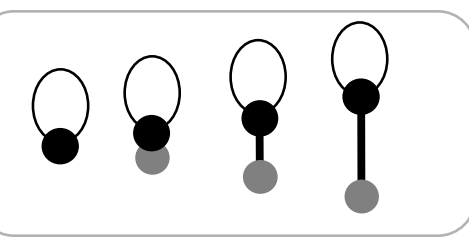

- die Schleife als Teil einer Mehrfachkante beide Knoten des $\mathrm{K}_{2}$ verbinden

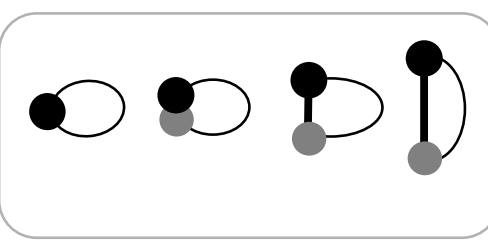

- eine mit dem ursprünglichen Knoten verbundene Kante mit ihm verbunden bleiben oder mit dem neuen Knoten verbunden sein.

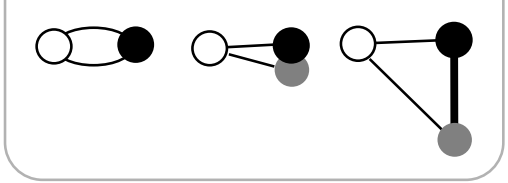


Aus dem Quotientengraphen (QG) des Netzes 1(8)1 können die Quotientengraphen aller sieben Minimalnetze der Ordnung 2 erzeugt werden. Abb. 4.1 zeigt zwei Beispiele.

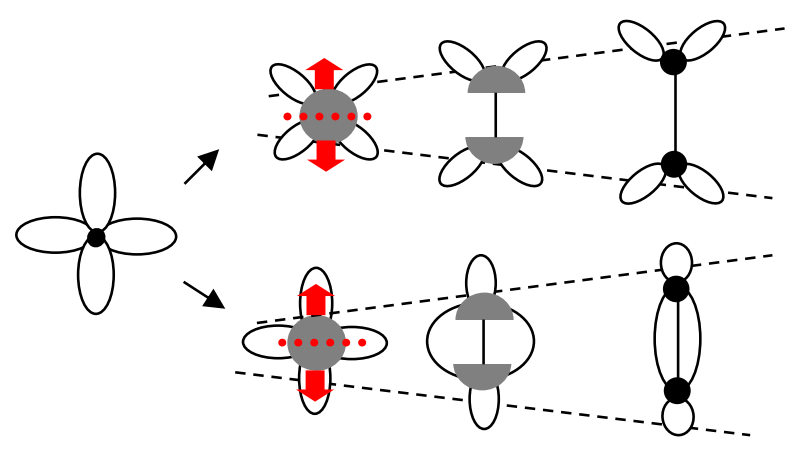

\begin{abstract}
Abb. 4.1 Bildung der QG der Netze 2(5)3 und 2(5)2 aus dem QG des Netzes 1(8)1. Der Knoten wird in seiner Mitte geteilt, die beiden Hälften werden zu eigenständigen Knoten und durch eine Kante verbunden. Oben: jede Knotenhälfte übernimmt zwei Schleifen, es entsteht der QG von 2(5)3. Unten: jede Knotenhälfte übernimmt eine Schleife und jeweils ein Ende der anderen beiden Schleifen, es entsteht der QG von 2(5)2.
\end{abstract}

Wie die Minimalnetze 2(5)3 und 1(8)1 auseinander hervorgehen, zeigt Abb. 4.2. Bei der Erhöhung der Ordnung wird aus jedem Knoten der Knotenklasse ein $K_{2}$ (beim Netz 1(8)1 gibt es nur eine Knotenklasse). Jeder der beiden Knoten, die dann zwei verschiedenen Knotenklassen angehören, übernimmt jeweils vier der acht Kanten des ursprünglichen Netzes.

\title{
4.2. Verringerung der Ordnung
}

Entsprechend umgekehrt können Quotientengraphen geringerer Ordnung erzeugt werden, in dem die Elemente des $\mathrm{K}_{2}$ - die beiden Knoten und die sie verbindende Kante - wieder zu einem Knoten verschmelzen (Abb. 4.2).

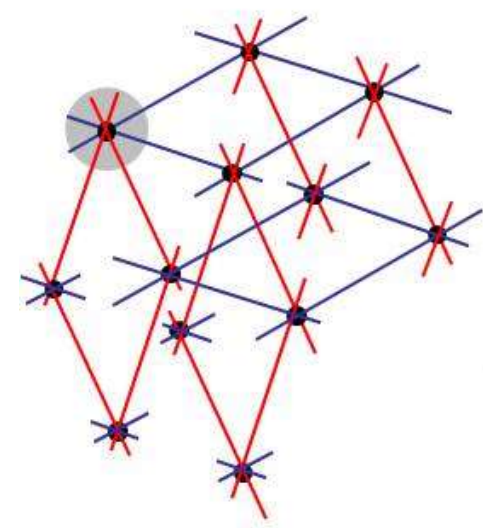

$1(8) 1$

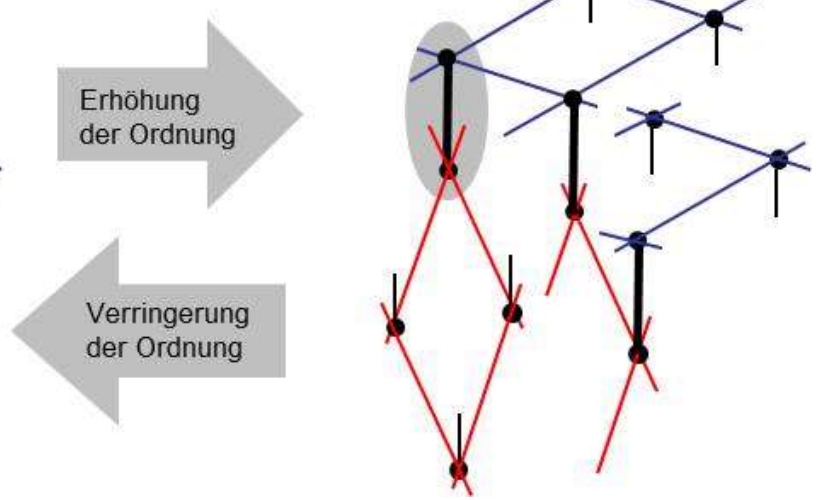

$2(5) 3$

Abb. 4.2 Minimalnetze 2(5)3 und 1(8)1 gehen auseinander hervor. Der mit der grauen Ellipse hinterlegte $\mathrm{K}_{2}$ in Netz 2(5)3 geht dabei aus dem mit grauem Kreis hinterlegten Knoten in Netz 1(8)1) hervor. Wie die QGs dieser beiden Netze auseinander hervorgehen, ist in der oberen Hälfte der Abb. 4.1 dargestellt. $\mathrm{K}_{2}$-Erzeugung $\rightarrow$ Erhöhung der Ordnung, $\mathrm{K}_{2^{-}}$,Verschmelzung“ $\rightarrow$ Verringerung der Ordnung. 
Aus dem Minimalnetz 6(3)1 mit dem Quotientengraphen $\mathrm{K}_{3,3}$ erhält man durch den Vorgang der Verringerung der Ordnung das Netz $5\left(3^{4}, 4\right) 1$.

In Abb. 4.3 werden die zwei Knoten, die sich innerhalb der gestrichelten Ellipse befinden (i), miteinander ,verschmolzen“. Dabei behalten beide Knoten ihre zu den anderen Knoten bestehenden Verbindungen. Die Kante, die die nun verschmolzenen Knoten verbunden hatte, „verschwindet“ (ii, iii). Der durch Verschmelzung entstandene Knoten hat den Knotengrad 4.

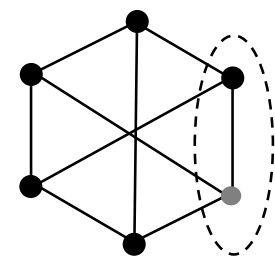

i
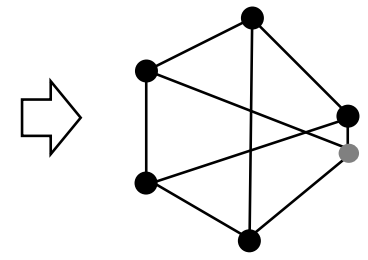

ii

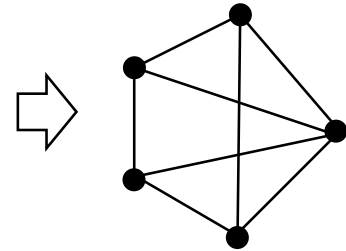

iii

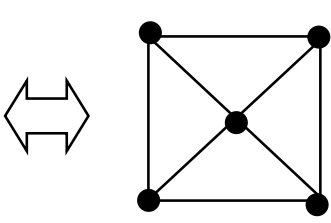

iv

Abb. 4.3 QG des Minimalnetzes 6(3)1 wird zu QG des Minimalnetzes 5(3 $\left.{ }^{4}, 4\right) 1$. Verschiebt man den rechten Koten der Skizze (iii) nach links, und verformt man das Gebilde ein wenig, so erhält man Skizze (iv). Die Skizzen (iii) und (iv) sind isomorphe Darstellungen desselben Graphen, denn ihre Knoten haben untereinander dieselben Nachbarschaftsrelationen.

Der Quotientengraph des Minimalnetzes $5\left(3^{4}, 4\right) 1$ hat acht Kanten, welche zwei verschiedenen Typen zugeordnet werden können (Abb. 4.4).

- Vier Kanten vom Typ ,a“ verbinden den Knoten mit Knotengrad 4 mit den vier anderen Knoten, die alle den Knotengrad 3 haben. Werden zwei Knoten, die an einer Kante dieses Typs liegen, miteinander verschmolzen, entsteht der Quotientengraph des Minimalnetzes $4\left(3^{3}, 5\right) 1$

- Die vier anderen Kanten vom Typ ,b` verbinden jeweils zwei Knoten, die beide Knotengrad 3 haben. Werden zwei Knoten, die an einer Kante dieses Typs liegen, miteinander verschmolzen entsteht der Quotientengraph des Minimalnetzes $4\left(3^{2}, 4^{2}\right)$

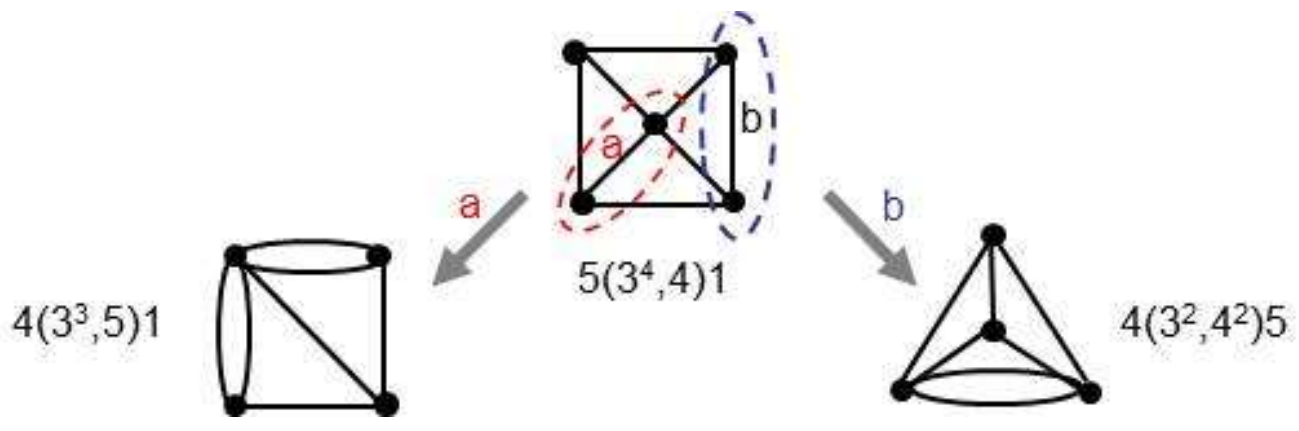

Abb. 4.4 Unterschiedliche Kantentypen im QG des Minimalnetzes 5(3 $\left.{ }^{4}, 4\right) 1$. Zwei Knoten eines Quotientengraphen, die an einer Kante vom Typ a bzw. b liegen, werden miteinander verschmolzen. Der mit a bzw. $\mathrm{b}$ beschriftete Pfeil zeigt auf den Quotientengraphen, der aus diesem Vorgang resultiert. 
Alle Minimalnetze lassen sich schrittweise durch Wiederholung dieses Vorgangs auf das vierperiodische Gitternetz - das Minimalnetz 1(8)1 - zurückführen (Abb. 4.5).

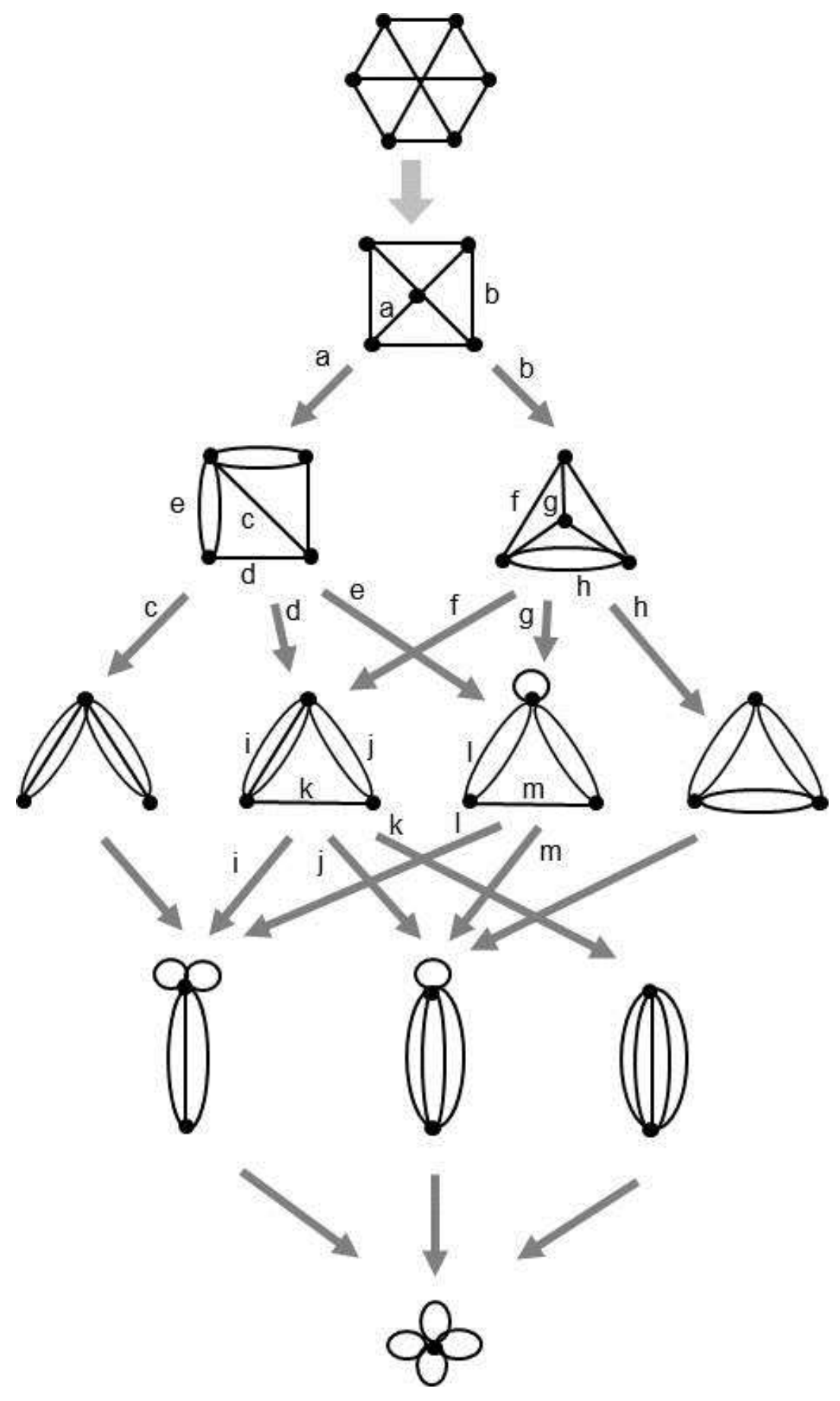

Abb. 4.5 Schrittweise Verringerung der Ordnung. Der mit , a (bzw. b,c,d,... $)^{\natural}$ beschriftete Pfeil zeigt auf den QG, der aus der Verschmelzung der zwei Knoten entsteht, die über eine Kante des entsprechenden Typs , a (bzw. b,c,d,...) verbunden waren. Pro Schritt wird die Ordnung des QG eines Minimalnetzes um 1 verringert. Wird mit einem QG der Ordnung 6 begonnen, entsteht nach fünf Schritten immer der QG des Netzes 1(8)1. 


\section{Erzeugung von 4p-Minimalnetzen aus 3p-Netzen mit gleichem Quotientengraphen}

Minimalnetze haben die Eigenschaft, dass sie bezüglich ihrer Nachbarschaftsrelationen eine 1:1-Relation zu ihrem Quotientengraphen haben. Bei vierperiodischen Netzen spannen vier dazu geeignete Kanten die vier Periodizitäten bzw. die vier Dimensionen (Vektorraum mit den Basisvektoren a, b, c, d) auf (Indizierung und Basisvektoren, siehe Kap. 2.2). Wird eine dieser vier Kanten umindiziert, indem ihr eine Linearkombination, bestehend aus den Basisvektoren der anderen drei Dimensionen, zugeordnet wird (z. B. d wird zu a+b), so entsteht ein dreiperiodisches Netz, welches nicht minimal ist. Die beiden Quotientengraphen unterscheiden sich nur in Bezug auf ihre Indizierung.

Dieser Vorgang lässt sich auch umkehren. Der Graph $\mathrm{K}_{4}$ - ein Tetraeder - ist ein 0-periodisches Netz (Abb. 5.1).

- Nun wird die blaue Kante zwischen C und A indiziert und baut eine (1-)periodische Struktur auf mit kürzesten Kreisen der Länge 3.

- Im nächsten Schritt bauen die Kanten DA (rot gestrichelt) und DC (gelb) gemeinsam eine Periodizität in einer zusätzlichen Richtung auf. Es entsteht ein zweiperiodisches Netz mit kürzesten Kreise der Länge 4 und zweitkürzesten Kreisen der Länge 8.

- Bauen diese beiden Kanten (DA und DC) nun zwei verschiedene Periodizitäten auf, erhält man das dreiperiodische Minimalnetz des $\mathrm{K}_{4}$, das Netz 4(3)1 mit kürzesten Kreisen der Länge 10. Da dieses Netz schon ein Minimalnetz ist, lässt sich aus dem $\mathrm{K}_{4}$ kein vierperiodisches Netz bilden.

\section{Konstruktion von periodischen Strukturen aus dem $\mathrm{K}_{4}$}

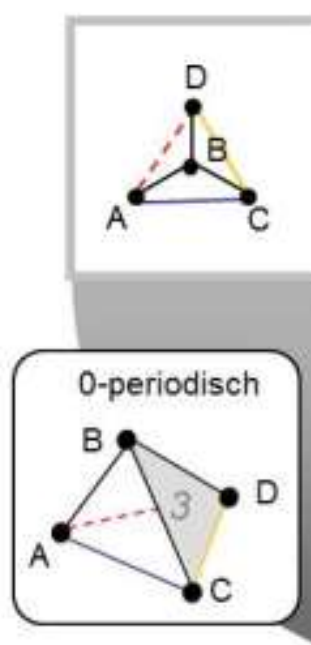

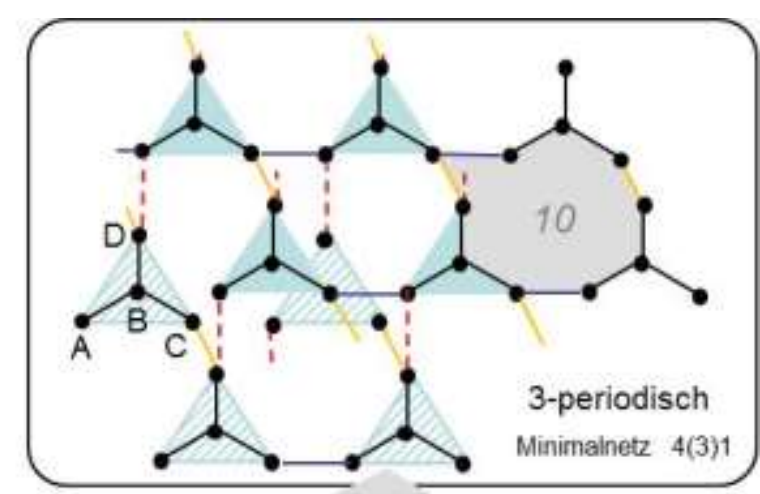

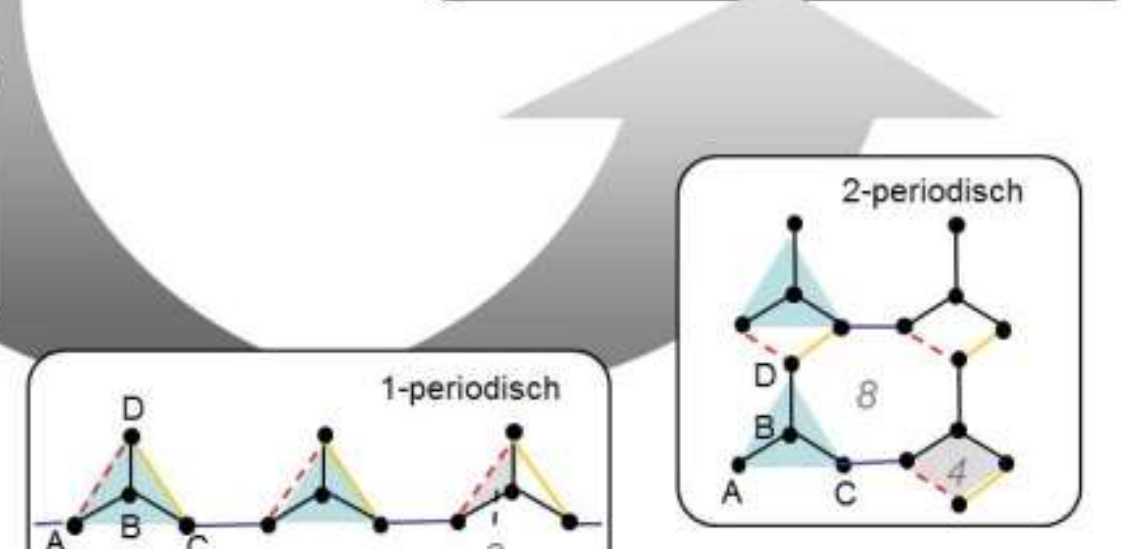

Abb. 5.1 Konstruktion des 3-periodischen Minimalnetzes 4(3)1 aus dem Tetraeder. 1-periodischer Graph: durch die blauen Kanten wird die erste Periodizität ,aufgebaut“. 2- periodischer Graph: durch die roten und gelben Kanten wird gemeinsam die zweite Periodizität aufgebaut. 3-periodisches Minimalnetz 4(3)1: durch die roten Kanten wird die zweite, und durch die gelben Kanten die dritte Periodizität aufgebaut. 


\subsection{Erzeugung des 4p-Gitternetzes}

Würde man in einem sog. Quadratnetz mit den Gitterkonstanten a und b (im Folgenden auch als 2p-Gitternetz bezeichnet) die Knoten auch in beiden diagonalen Richtungen miteinander verbinden, entstünde ein zweiperiodisches Netz mit Knoten, die alle den Knotengrad 8 haben und einer einzigen Knotenklasse angehören. Sein Quotientengraph bestünde dann aus einem Knoten und vier Schleifen mit folgender Indizierung:

$\rightarrow \quad 10$ (bzw. a), 01 (bzw. b), 11 (bzw. a b), und -11 (bzw. -a b).

Ein solches Netz lässt sich in keinen Raum einbetten. Mit gekrümmten Kanten wäre allerdings eine kreuzungsfreie Realisierung dieses Netzes im 3-dimensionalen Raum darstellbar (Abb. 5.2).

Durch Umindizierung der Schleife ,ab“ in „c“ (wobei c linear unabhängig von a und b ist) entsteht nun ein 3-periodisches Netz.

Wird nun die Schleife ,-ab“ in d umindiziert (wobei d linear unabhängig von $a, b$ und c ist), entsteht ein 4-periodisches Minimalnetz, das 4-periodische Gitternetz 1(8)1.
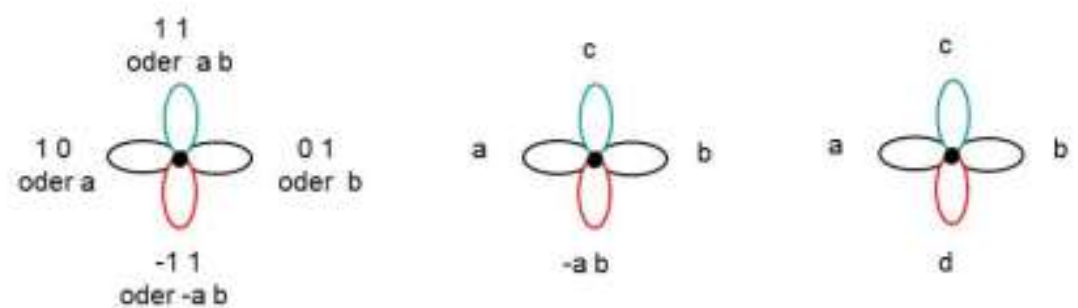

oder - $\mathrm{a} b$

ab

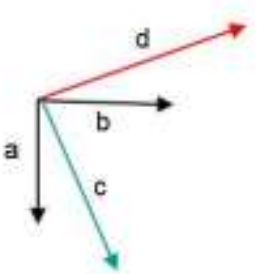

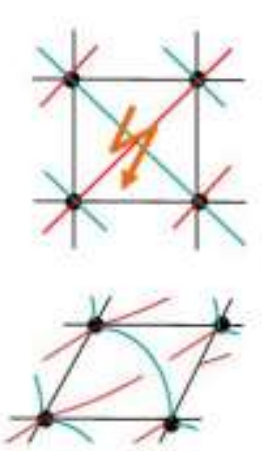

2periodisch

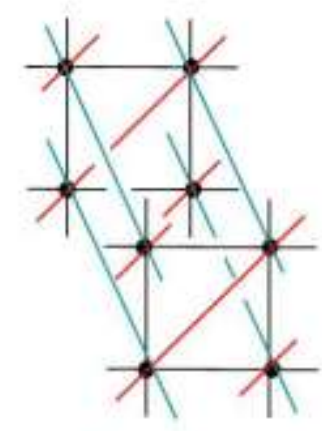

3 periodisch

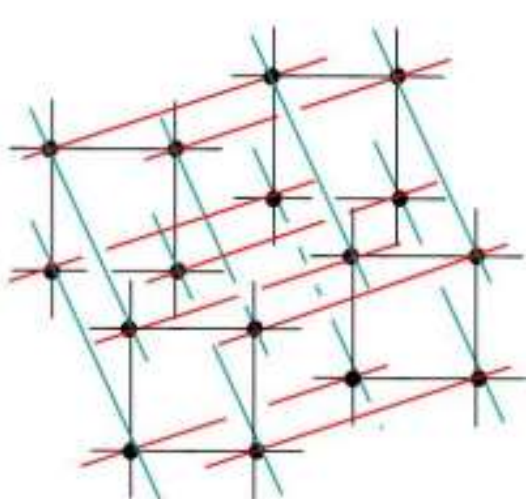

4periodisch

Abb. 5.2 Entstehung des 4p-Gitternetzes durch Umindizierung von Kanten. Die Kanten des 2periodischen Netzes in dieser Abbildung überkreuzen (berühren) sich (orangener Blitz in Darstellung), darunter Darstellung mit gekrümmten Kanten). 


\subsection{Erzeugung des 4p-Minimalnetzes 6(3)2}

Bei einem dreieckigen Prisma bilden die Ecken zusammen mit den Kanten den Graphen $\mathrm{K}_{2} \times \mathrm{K}_{3}$, (siehe [Harary, 1969] und Abb. 5.3). Das Minimalnetz dieses Graphen ist das 4-periodische Minimalnetz 6(3)2.
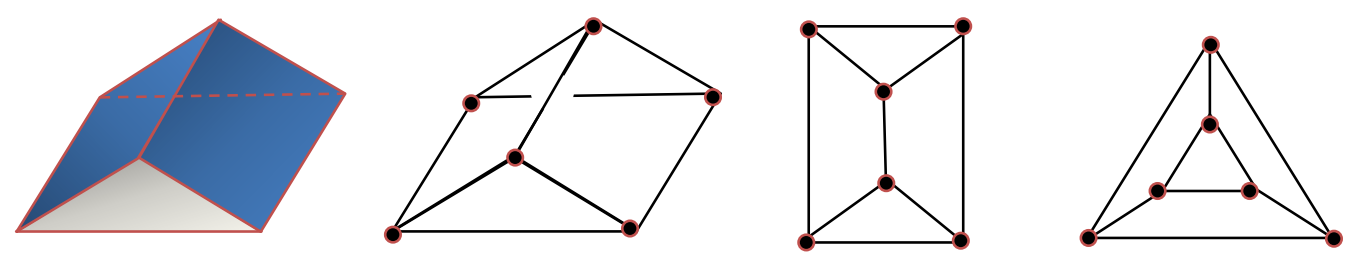

Abb. 5.3 Dreieckiges Prisma und drei Darstellungen des Graphen $K_{2} \times K_{3}$. Die drei Darstellungen des Graphen sind zueinander isomorph (vgl. auch mit Abb. 2.2). In Tabelle 3.1 sind die Periodenlängen der 1-periodischen Ketten des dazugehörigen Minimalnetzes aufgeführt.

Durch entsprechende Indizierung der Kanten können aus diesem Graphen verschiedene 3-periodische Netze generiert werden. Exemplarisch werden nun die vier Netze betrachtet, deren kürzeste Kreise maximal die Länge 6 haben. Ihre Kreisklassenspektren sind in Tab. 5.1 dargestellt, incl. ungerader Längen, da die 3p-Netze dieses Graphen keine Minimalnetze sind.

\begin{tabular}{lcccccccccccc} 
Kreislänge & 3 & 4 & 5 & 6 & 7 & 8 & 9 & 10 & 11 & 12 & 13 & 14 \\
\hline 3p-Netz Nr.1 & $\mathbf{1}$ & 0 & 0 & 0 & 0 & 0 & 0 & 0 & 0 & 3 & 6 & 8 \\
3p-Netz Nr.2 & 0 & $\mathbf{1}$ & 0 & 0 & 0 & 0 & 0 & 0 & 0 & 8 & 0 & 21 \\
3p-Netz Nr.3 & 0 & 0 & $\mathbf{1}$ & 0 & 0 & 0 & 0 & 0 & 3 & 5 & 3 & 12 \\
3p-Netz Nr.4 & 0 & 0 & 0 & $\mathbf{1}$ & 0 & 0 & 0 & 2 & 0 & 8 & 0 & 18
\end{tabular}

$4 \mathrm{p}-\operatorname{Min} 6(3) 2-0 \quad-0-0 \quad-0-0-6-15$

Tab. 5.1 $K_{2} \times K_{3}$ : Kreisklassen der vier 3p-Netze und des $4 p$-Minimalnetzes. Kreise ungerader Länge gibt es in Minimalnetzen gemäß Definition nicht. Diese Tatsache wird durch das Symbol ‘.' repräsentiert.

In Abb. 5.4 (folgende Seite) sind die Quotientengraphen durch Farbgebung indiziert, wobei verschiedene Farben für den Aufbau verschiedener Periodizitäten stehen.

Anbei ein Beispiel für eine Zuordnung: $\operatorname{rot} \rightarrow \mathrm{a}$, orange $\rightarrow \mathrm{b}$, grün $\rightarrow \mathrm{c}$, blau $\rightarrow \mathrm{d}$. (a entspricht einer Indizierung von $100 \mathrm{im} \mathrm{3p-Netz} \mathrm{und} \mathrm{von} 1000 \mathrm{im} \mathrm{4p-Netz)}$

Darunter befindet sich in Abb. 5.4 jeweils das dazugehörige dreiperiodische Netz. Die kürzesten Kreise sind jeweils grau eingefärbt.

In der Mitte der Grafik wird bei jedem der vier Netze eine zu seinem kürzesten Kreis gehörende Kante mit der in dem Netz noch fehlenden Farbe markiert (bzw. indiziert). Bei Netz Nr.1 kommt also die Farbe blau dazu (Kante von E nach C). Diese Kante wird ,aufgetrennt“" und spannt nun die 4. Periodizität auf.

Führt man diese Aktion auf analoge Weise bei den anderen drei Netzen durch, so erhält man jedes Mal dasselbe Netz: es ist das 4-periodische Minimalnetz 6(3)2. 

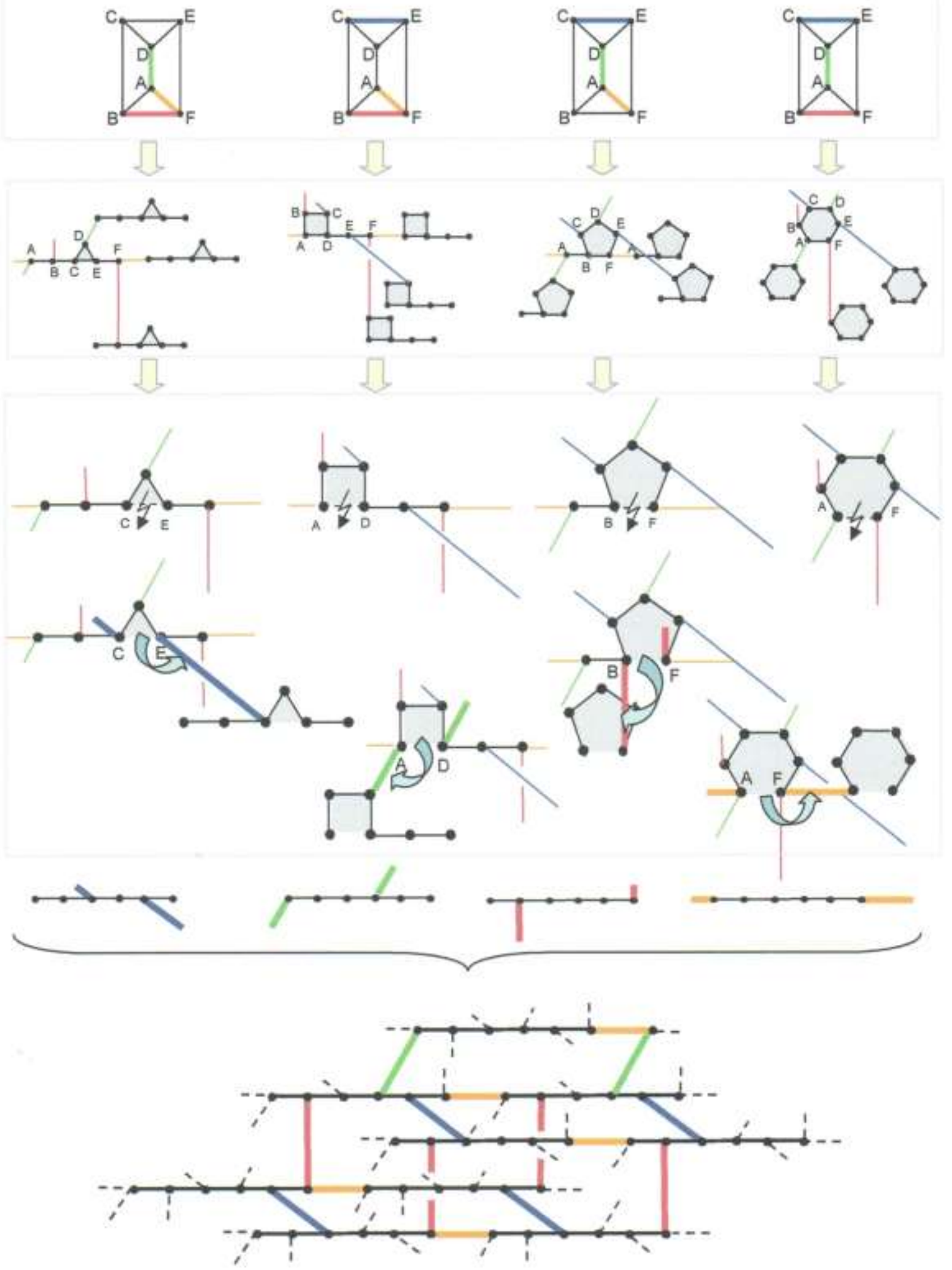

Abb. 5.4 4p-Minimalnetz aus 3p-Netzen mit demselben Quotientengraphen. Oben die hier durch Farben indizierten QG derjenigen 3p-Netze des $\mathrm{K}_{2} \times \mathrm{K}_{3}$, deren Kreisklassen in Tab. 5.1 aufgelistet sind, und darunter Ausschnitte aus ihren Netzen. In der Mitte wird bei allen Netzen jeweils eine Kante(nklasse), die nicht für den Zusammenhalt des Netzes gebraucht wird (denn diese 3p-Netze sind keine Minimalnetze) dazu verwendet, die vierte Periodizität aufzubauen. Aus allen vier 3p-Netzen entsteht dabei das 4p-Minimalnetz 6(3)2. 


\section{Konstruktionsprinzipien}

4-periodische Minimalnetze lassen sich prinzipiell auf sehr vielfältige Weise konstruieren. Um topologische Unterschiede zweier Netze graphisch verständlich zu machen, sind an eine Konstruktion andere Kriterien anzulegen, als wenn geometrische Aspekte im Vordergrund stehen, also eine geeignete Einbettung in einen entsprechenden Raum gesucht wird.

Methoden zur Konstruktion sind:

- Substrukturanalyse (Kap . 3)
○ Bäume
○ Ketten
- Kreisklassen
○ Subnetze

- Betrachtung ähnlicher Netze, z. B.

$\circ \quad 4 p-N e t z e$ anderer Ordnung (Kap. 4)

$\circ$ 3p-Netze mit gleichem Quotientengraphen (Kap. 5)

- Geometrische Optimierung

○ Knotenabstände

○ Kantenlängen

○ Winkel

Für das Netz 3(4)1 ist in Abb. 6.1 eine Darstellung gewählt, bei der die drei Ketten der Periodenlänge 2 und Vertreter von drei (der 15) Kreisklassen mit Kreisen der Länge 8 gut zu erkennen sind. Werden in den Ketten mit PL=2 alle Knoten der einen Knotenklasse (gegenüber denen der anderen Knotenklasse) auf gleiche Weise verschoben, so nehmen die Ketten die Form einer Zickzacklinie an.

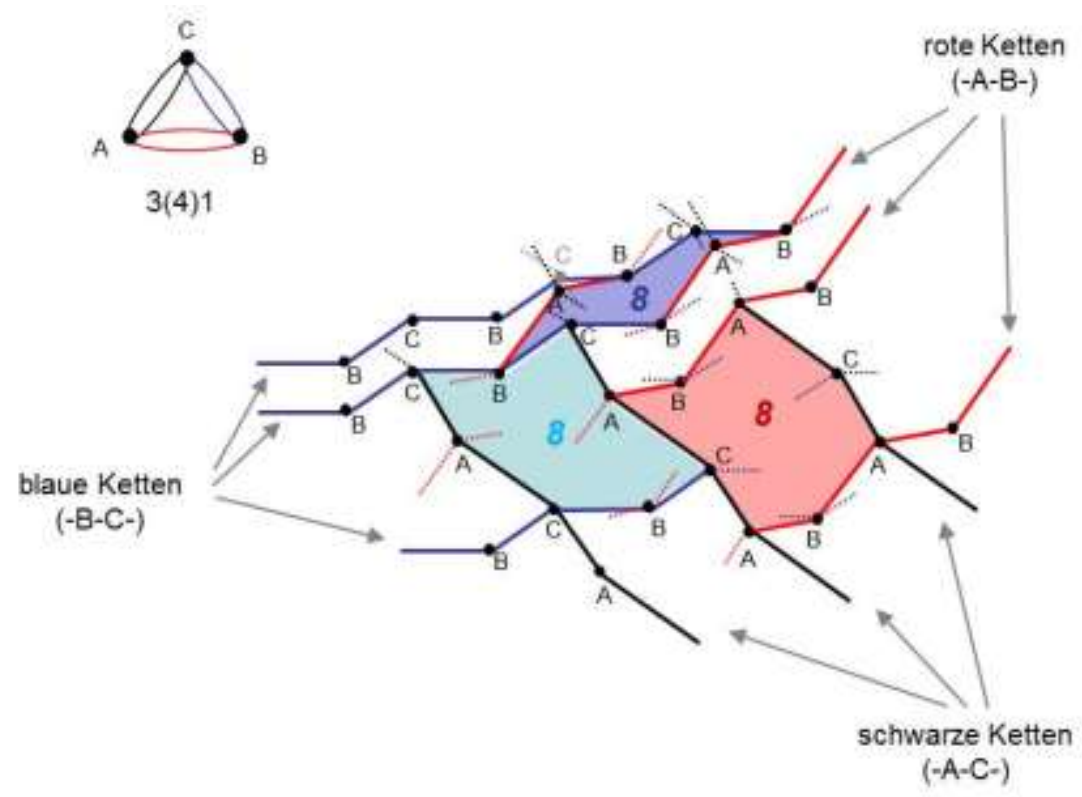

Abb. 6.1 Ketten und Kreise des Minimalnetzes 3(4)1. Durch Kombination zweier Kettenscharen lassen sich 2-periodische Subnetze bilden. Z. B. bilden über die Knoten A verbundenen rote (-A-B-) und schwarze Ketten (-A-C-) eine 2-periodische Substruktur mit Kreisen der Kantenfolge (-A-B-A-C-A-B-A-C-), ein Vertreter davon ist der rote Kreis in der Abbildung. 


\subsection{Darstellung des Minimalnetzes $5\left(3^{4}, 4\right) 4$}

In Abb. 6.2 auf der folgenden Seite sind zwei unterschiedliche Konstruktionsmöglichkeiten des Minimalnetzes $5\left(3^{4}, 4\right) 4$ dargestellt.

Das Minimalnetz 5(3 $\left.3^{4}, 4\right) 4$ hat folgendes Kreisklassenspektrum:

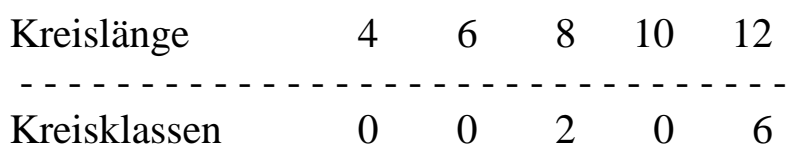

Ausgangspunkte für die Konstruktion des Netzes sind

- Konstruktion in Abb. 6.2 oben:

einer der drei Bäume (siehe auch Abb. 3.3 und 3.5) mit fünf Knoten, in Abb. 6.2 auf grauer Ellipse dargestellt.

Bei dieser Konstruktion sind die vier Substrukturen, die aus 12er-Kreisen von vier (der sechs) Kreisklassen mit Kreisen der Länge 12 gebildet werden, topologisch gleichwertig. Einer dieser Kreise ist durch die blaue Fläche gekennzeichnet.

- Konstruktion in Abb. 6.2 unten:

die 2-periodischen Substrukturen, die durch zwei Kreisklassen mit den Kreisen der Länge 8 (orangene Flächen) gebildet werden, sind jeweils über den Knoten A verbunden.

Zwei Kreisklassen mit Kreisen der Länge 12 (gelbe Flächen) lassen sich durch die Umrandung zweier benachbarter Achterkreise bilden. Vertreter der übrigen vier Kreisklassen mit den Kreisen der Länge 12 sind rechts dargestellt. Das Netz ist so konstruiert, dass die blauen 12er-Kreise Schaaren aus 2-periodischen Substrukturen bilden, die in Ebenen liegen. 


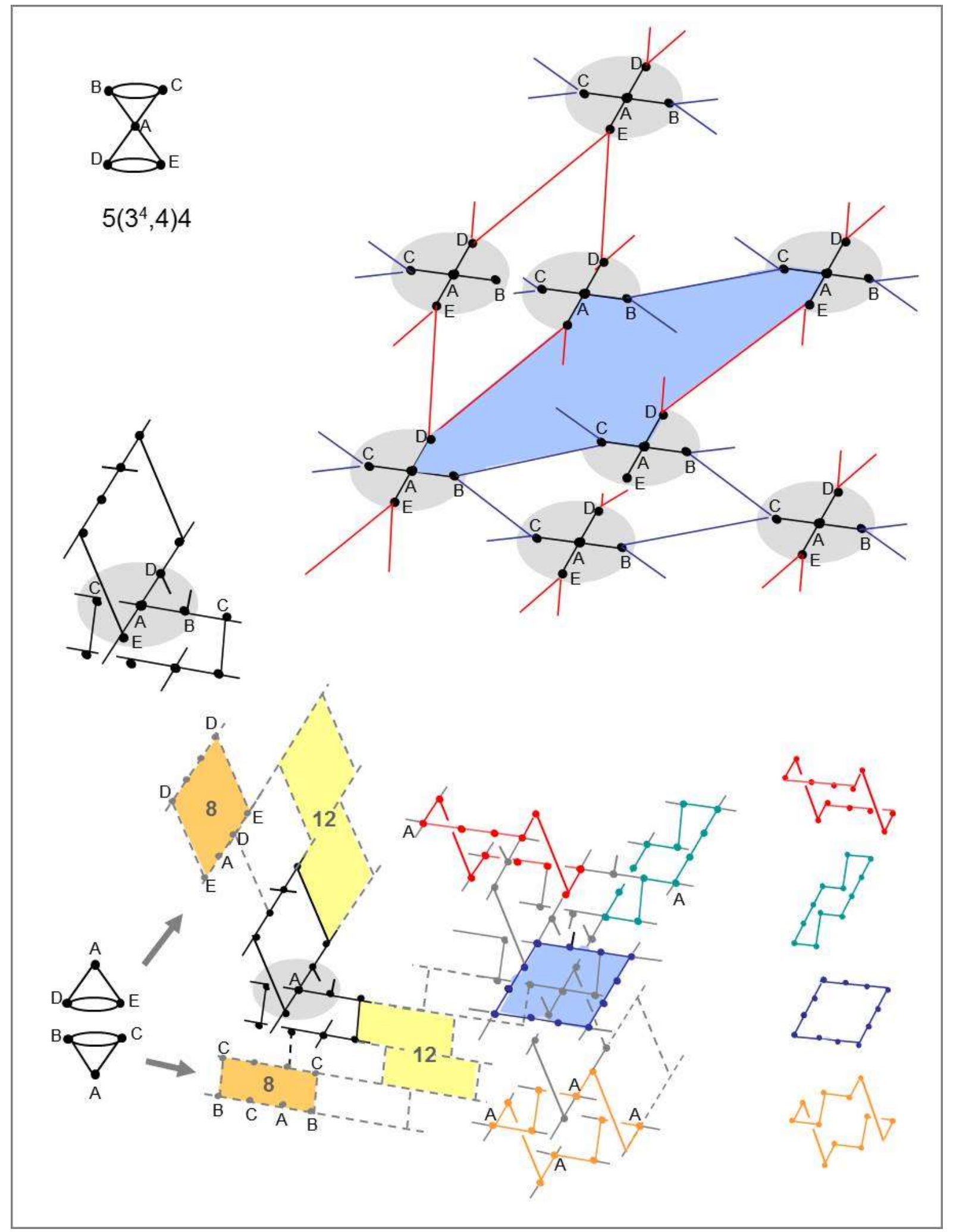

Abb. 6.2 Zwei Konstruktionen des Minimalnetzes 5(34)4. Oben links der QG dieses Netzes. Oben rechts: Konstruktion, ausgehend vom untersten Baum des Bildbereichs $\mathrm{n}=5$ aus Abb. 3.3; die Bäume sind durch graue Ellipsen hinterlegt, ein 12er-Kreis wird durch die blaue Fläche verdeutlicht. Unten rechts sind zwei topologisch gleichwertige Teilgraphen gezeigt und die aus ihnen resultierenden Kreisklassen im Netz (daneben): pro Teilgraph gibt es je eine Kreisklasse von 8er- (orange) und eine von 12er-Kreisen (gelb). Die aus 8er-Kreisen bestehenden 2-periodischen Substrukturen sind über Knoten des Typs A miteinander verbunden. Vertreter der vier anderen Kreisklassen der Länge 12 sind unten in der Mitte und unten rechts dargestellt. 


\subsection{Darstellung des Minimalnetzes 6(3)3}

Das Minimalnetz 6(3)3 ist in Abb. 6.3 dargestellt. Es hat folgendes Kreisklassenspektrum:

\begin{tabular}{lcccccc} 
Kreislänge & 4 & 6 & 8 & 10 & 12 & 14 \\
\hdashline Kreisklassen & 0 & 0 & 0 & 1 & 2 & 14
\end{tabular}

Die aus 10er-Kreisen gebildeten 2-periodischen Substrukturen sind Ausgangspunkt zur Konstruktion des Minimalnetzes 6(3)3. Das graue (,gekachelte“) Muster stellt ein Beispiel für eine solche Substruktur dar, wenn sie in die Ebene eingebettet ist.

Die Doppelkante zwischen E und F bedeutet, dass im Netz Ketten der Periodenlänge 2 vorhanden sind. Die hellgraue dicke Linie verdeutlicht, dass diese Kette 1-periodisch ist.

Zwei Kantenklassen (Kanten zwischen A und E sowie Kanten zwischen C und F) verbinden diese beiden unterschiedlichen Substrukturen.

Durch Verschieben der Knoten

- A nach unten und E nach oben

- C nach oben und F nach unten

können die Knotenabstände optimiert werden.

Vertreter der Kreisklassen der Länge 10, 12 und 14 sind in Abb. 6.3 skizziert. Die vierzehn Kreisklassen der Länge 14 ergeben sich wie folgt:

- Teilstruktur des Quotientengraphen unten links: 2x4 = 8 Kreisklassen.

Die Vertreter zweier Kreisklassen sind dargestellt.

Werden in dieser Struktur anstatt der Kante zwischen A und B die

Kante $\mathrm{AD}$, oder $\mathrm{DC}$, oder $\mathrm{BC}$ weggelassen, erhält man die übrigen 6 Kreisklassen.

- Teilstruktur des Quotientengraphen unten in der Mitte: 2x2=4 Kreisklassen

Zwei Kreisklassen sind durch die gezeigte Teilstruktur des Quotientengraphen gegeben, indem bei gleicher Startkante (obere Kante der Doppelkante) die Kantenfolge nach dem Knoten A variiert (E-F-E-A-D... und E-F-E-A-B...),

die anderen beiden erhält man für die gespiegelte Teilstruktur (Knoten C statt Knoten A).

- Teilstruktur des Quotientengraphen unten rechts: 2 Kreisklassen.

Die andere Kreisklasse erhält man, wenn die Kanten AB und DC gelöscht werden und durch die Kanten AD und BC ersetzt werden. 


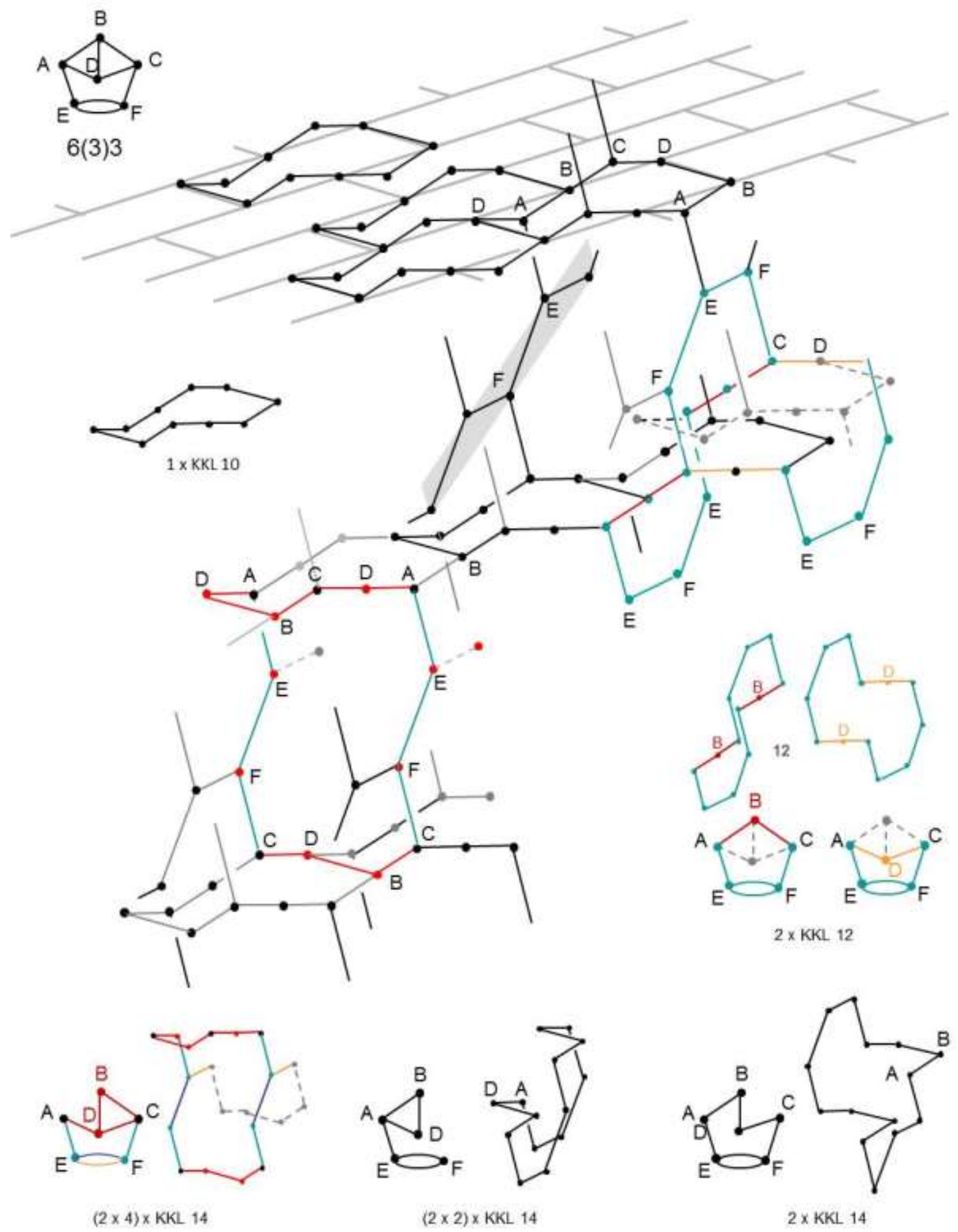

Abb. 6.3 Kreisklassen des Minimalnetzes 6(3)3 bis zur Länge 14. 2-periodische Substrukturen aus 10er-Kreisen (Knoten $B$ und $D$ je 3x, A und $C$ je 2x im Kreis) werden über Ketten der PL=2 (Knoten $\mathrm{E}$ und $\mathrm{F}$ ) miteinander verbunden, wobei $\mathrm{A}$ mit $\mathrm{E}$ und $\mathrm{F}$ mit $\mathrm{C}$ verbunden ist. 


\section{Die vierperiodischen Minimalnetze}

Besondere Eigenschaften von periodischen Netzen, wie äquivalente Knoten oder Kanten, sind von großer Bedeutung für die Zuordnung von Kristallstrukturen zu Strukturtypen [Blatov, Proserpio, 2011]. Strukturen, bei denen alle Kanten äquivalent sind, werden kantentransitiv genannt. 3-periodische kantentransitive Netze wurden von [Delgado-Friedrichs, O'Keeffe, Yaghi, 2006] untersucht. Unter den 4-periodischen Minimalnetzen gibt es vier kantentransitive Netze.

Neben der Anzahl der vierperiodischen Minimalnetze pro Typ und Ordnung sind in der Tabelle 7.1 die Quotientengraphen mit äquivalenten Knoten und Kanten aufgeführt. Die Nomenklatur der Quotientengraphen der 111 vierperiodischen Minimalnetze ist aus [Beukemann, Klee, 1992] übernommen.

\begin{tabular}{|c|c|c|c|c|}
\hline \multicolumn{2}{|c|}{ Ordnung, Typ } & \multirow{2}{*}{$\begin{array}{c}\text { Anzahl } \\
1\end{array}$} & \multirow{2}{*}{$\begin{array}{l}\begin{array}{l}\text { Aquivalente } \\
\text { knoten und } \\
\text { Kanten }\end{array} \\
\end{array}$} & \multirow[t]{2}{*}{$\begin{array}{l}\text { Aquivalente Knoten, } \\
2 \text { Kantentypen }\end{array}$} \\
\hline 1 & $1(8)$ & & & \\
\hline 2 & $\begin{array}{l}2(5) \\
2(3,7) \\
2(4,6)\end{array}$ & $\left.\begin{array}{l}3 \\
2 \\
2\end{array}\right\}$ & & $\infty$ \\
\hline 3 & $\begin{array}{l}3(4) \\
3\left(3^{2}, 6\right) \\
3(3,4,5)\end{array}$ & $\left.\begin{array}{r}4 \\
6 \\
10\end{array}\right\} 20$ & & \\
\hline 4 & $\begin{array}{l}4\left(3^{3}, 5\right) \\
3\left(3^{2}, 4^{2}\right)\end{array}$ & $\left.\begin{array}{l}14 \\
22\end{array}\right\} 36$ & & \\
\hline 5 & $5\left(3^{4}, 4\right)$ & 30 & & \\
\hline 6 & $6(3)^{\prime}$ & 17 & $\Delta \Delta$ & \\
\hline & & 111 & & \\
\hline
\end{tabular}

Tab. 7.1 Anzahl der Minimalnetze pro Typ und Ordnung, und Quotientengraphen mit äquivalenten Knoten und Kanten. Die maximale Ordnung der QG von 4p-Minimalnetzen beträgt 6 .

Es gibt keine vierperiodischen Minimalnetze mit Quotientengraphen einer höheren Ordnung als 6 aufgrund der für Minimalnetze gültigen Beziehung $\mathrm{p}=\mathrm{e}-(\mathrm{v}-1)$ mit $\mathrm{p}=$ Periodizität, $\mathrm{e}=$ Anzahl der Kanten (edges) und $\mathrm{v}=$ Anzahl der Knoten (vertices).

Die maximale Ordnung $\mathrm{v}_{\max }$ eines Quotientengraphen eines Minimalnetzes der Periodizität $\mathrm{p}$ lässt sich aufgrund der Zusatzbedingung e $=3 / 2 \mathrm{v}$ (der minimale Knotengrad beträgt 3, und eine Kante verbindet 2 Knoten) wie folgt ermitteln: $v_{\max }=2 p-2$.

D. $\mathrm{h}$ für $\mathrm{p}=4$ ist $\mathrm{v}_{\max }=6$. 


\subsection{Das 4-periodische Gitter - Minimalnetz 1(8)1}

Analysiert man die Kreisklassen der Kreislänge 6 der 2-, 3- und 4-periodischen (und -dimensionalen) Gitternetze, so sind folgende Zusammenhänge sichtbar:

- Die zwei 6er-Kreisklassen des ebenen 2p-Gitternetzes finden sich jeweils in den drei 2-dimensionalen Unterräumen des räumlichen 3p-Gitternetzes wieder.

- $\quad$ Im 3p-Gitternetz lassen sich zusätzlich 6er-Kreise finden, die alle 3 Raumrichtungen in Anspruch nehmen. Zwölf Kreisklassen umranden zwei aneinander liegende Quadrate mit zwei senkrecht aufeinander stehenden Flächennormalen. Vier weitere Kreisklassen umranden drei Quadrate, die paarweise aneinander liegen, wobei ihre Flächendiagonalen in drei Raumrichtungen zeigen. Diese 16 Kreisklassen finden sich in allen vier 3dimensionalen Unterräumen des 4p-Gitternetzes wieder (Abb. 7.1).

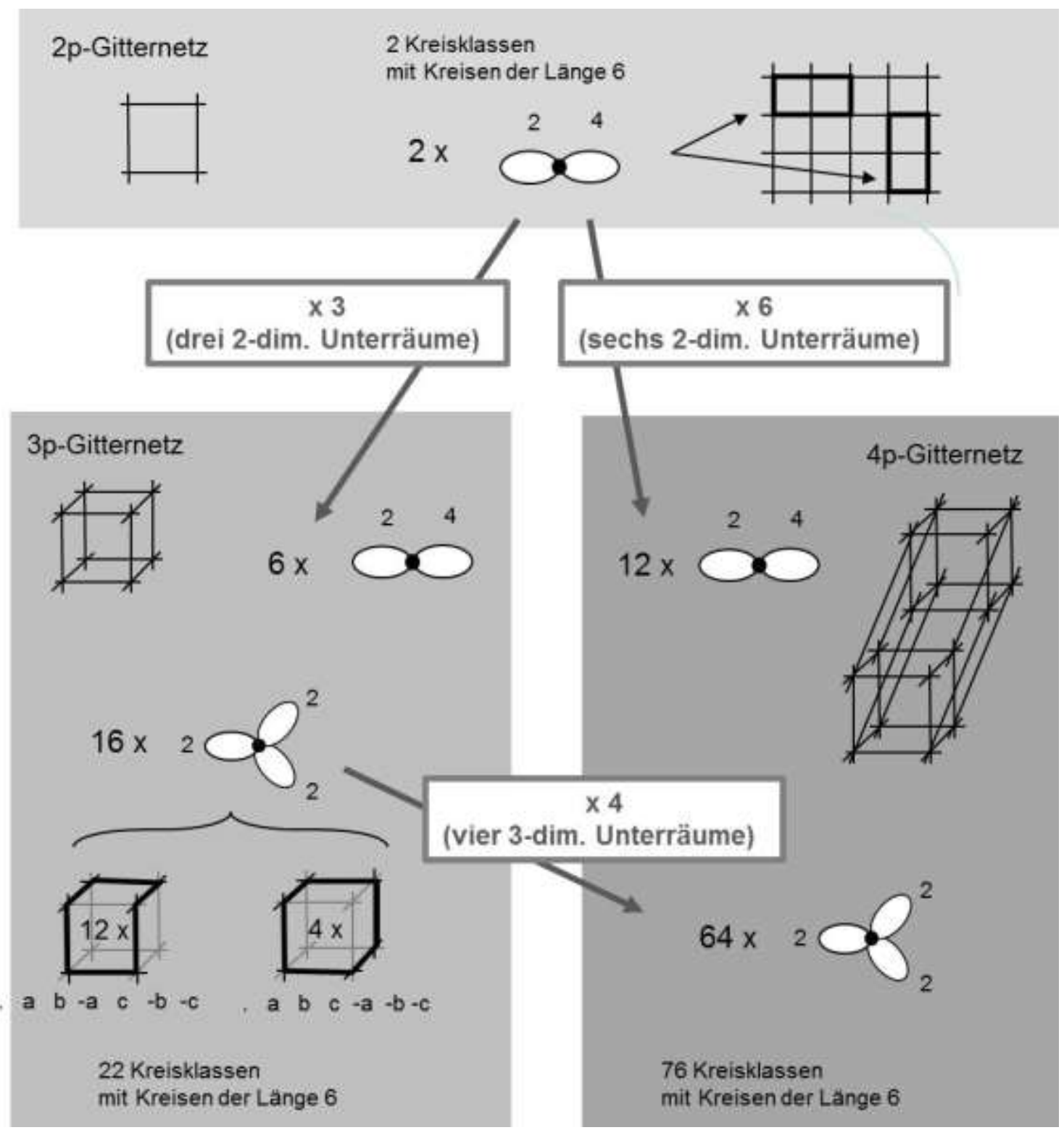

Abb. 7.1 2p-, 3p- und 4p-Gitternetz. Unterräume und Kreisklassen mit Kreisen der Länge 6. 


\subsection{Analyse von zwölf 4-periodischen Minimalnetzen}

In Abb. 7.3 bis 7.14 werden zwölf 4p-Minimalnetze (das der Ordnung 1, alle sieben der Ordnung 2, sowie vier der Ordnung 6) detailliert beschrieben. Die Beschreibungen enthalten jeweils die Bezeichnung, den Quotientengraphen, eine perspektivische Darstellung sowie einen Hinweis zur einer möglichen Konstruktion des Netzes. Darüber hinaus sind alle möglichen (1-periodischen) Ketten, die im Netz enthaltenen Minimalnetze geringerer Periodizität, und die Kreisklassen, untergliedert nach ihren Typen, aufgeführt (Abb. 7.2).

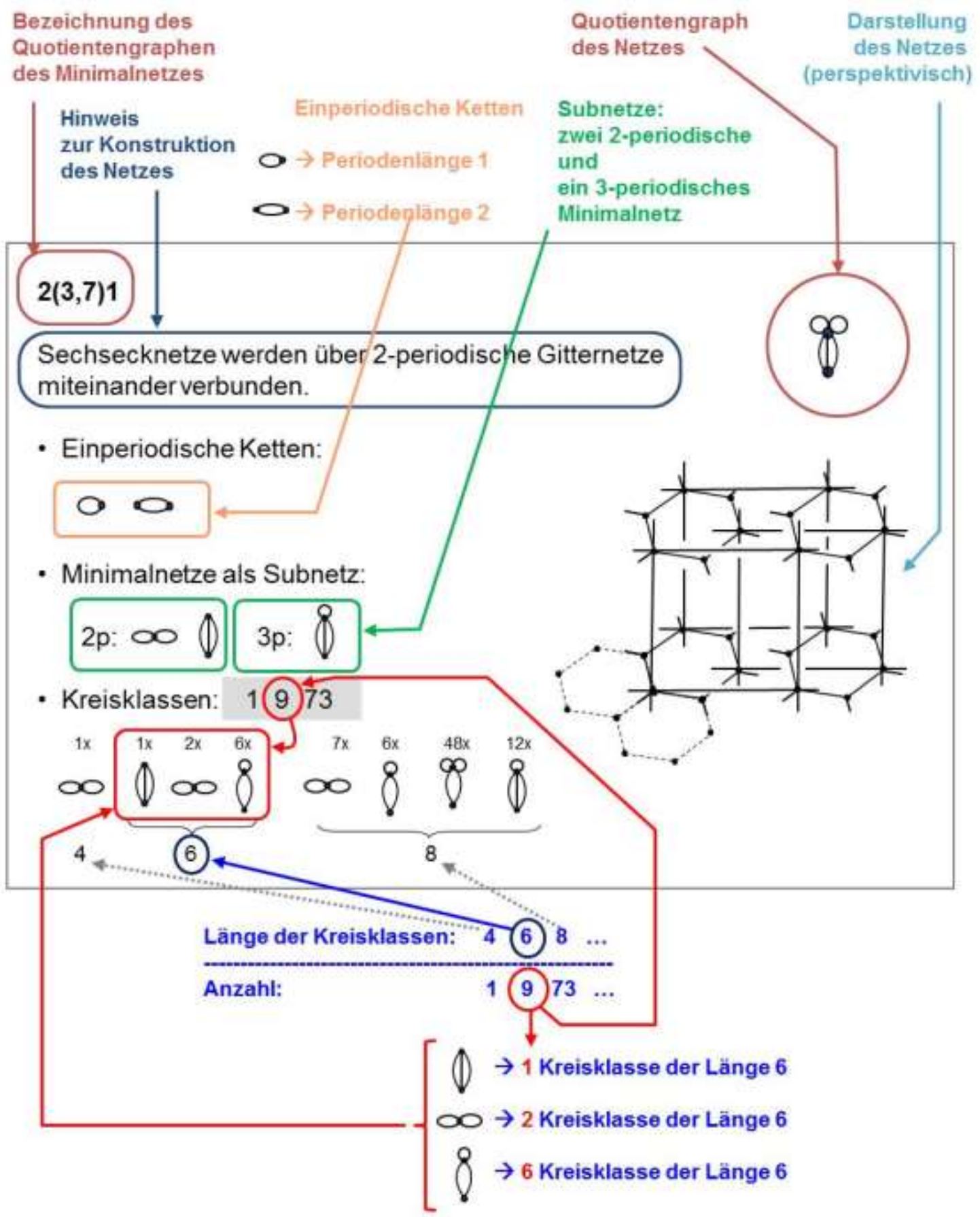

Es gibt in diesem Beispiel 9 Kreisklassen der Lănge 6 , sie lassen sich Substrukturen mit unterschiedlichen Quotientengraphen zuordnen.

Abb. 7.2 Erläuterung zu den Abbildungen 7.3 bis 7.14 


\section{1(8)1}

Vierperiodisches Gitternetz

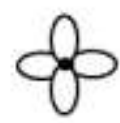

- Einperiodische Ketten:

- Minimalnetze als Subnetz:

$2 p: \infty$ 3p: of

- Kreisklassen: 676
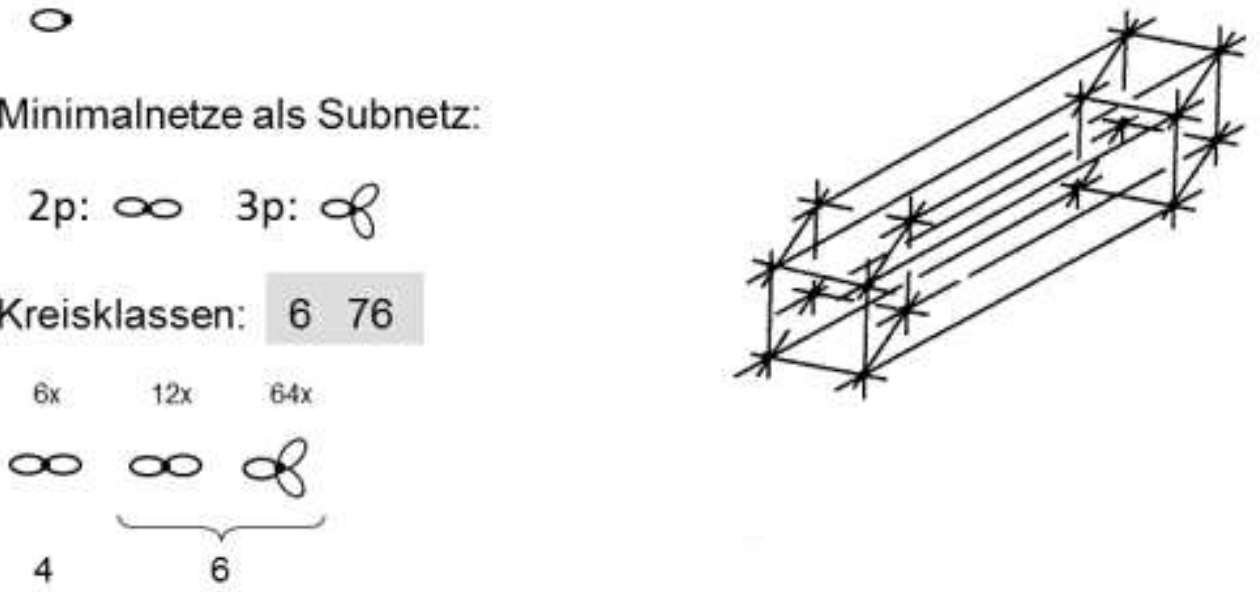

Abb. 7.3 Minimalnetz 1(8)1

\section{2(5)1}

Vierperiodisches Diamantnetz

(Hyperdiamantnetz)

- Einperiodische Ketten:

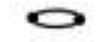

- Minimalnetze als Subnetz:

$$
\text { 2p: 3p: }
$$

- Kreisklassen: $0 \quad 1030$
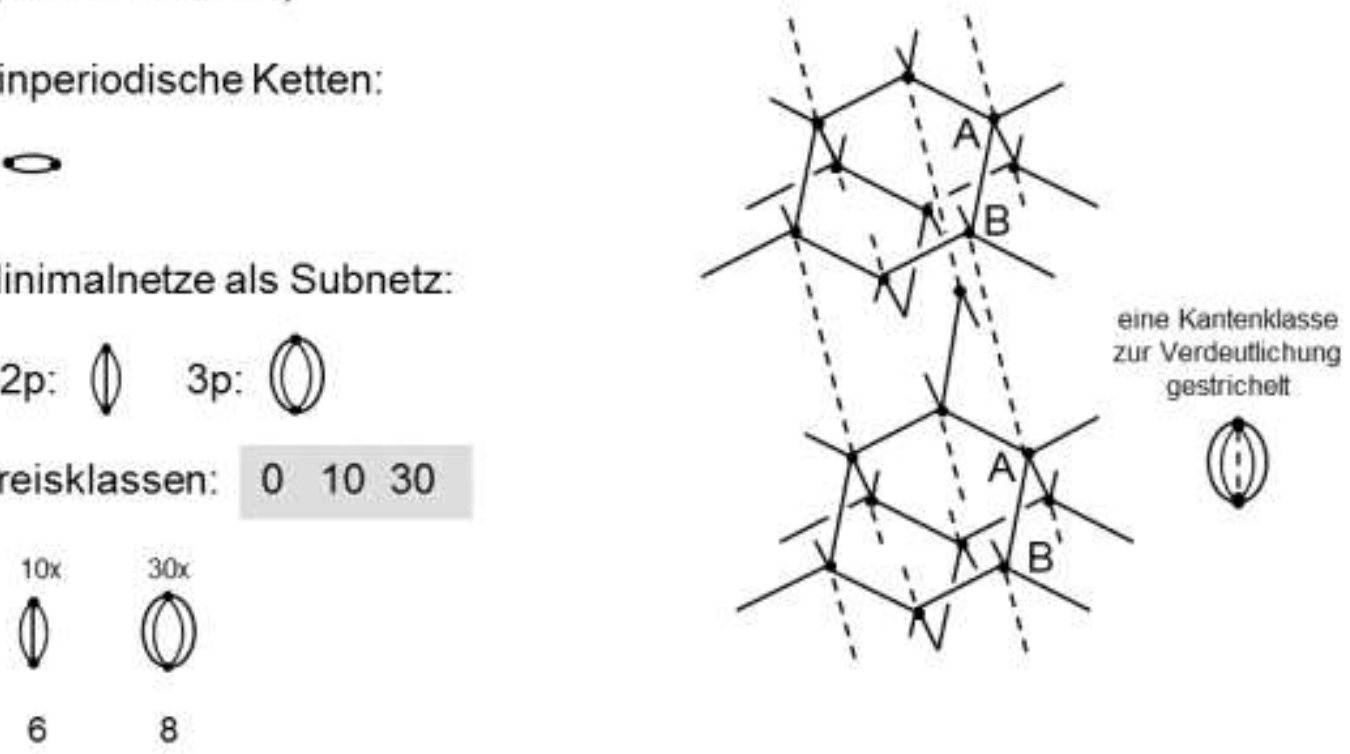

Abb. 7.4 Minimalnetz 2(5)1 


\section{2(5)2}

2-periodische Minimalnetze (Sechsecknetze), verbunden durch 2 Kettenscharen mit jeweils der Periodenlänge 1.

- Einperiodische Ketten:

- Minimalnetze als Subnetz:

$$
\text { 2p: } 1 \text { 3p: }
$$

- Kreisklassen: 0733

$$
\underbrace{1 x}_{6} 8_{8}^{6 x} \underbrace{3 x}_{0} 8^{12 x} 8^{6 x} 8^{12 x}
$$

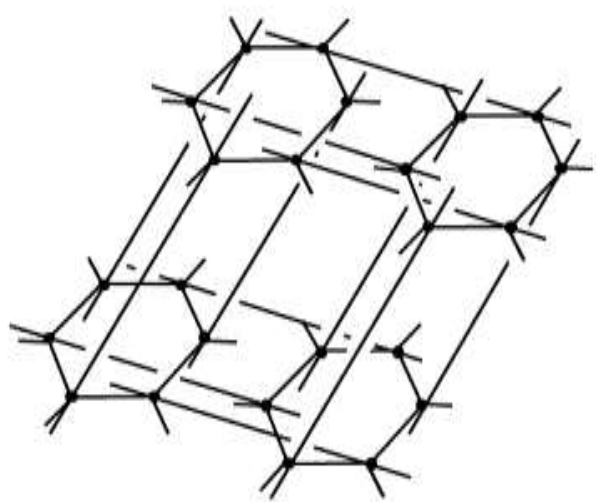

Abb. 7.5 Minimalnetz 2(5)2

\section{$2(5) 3$}

2-periodische Gitternetze (2 Scharen) in jedem Knoten über eine Kante mit einem Netz der anderen Schar verbunden.

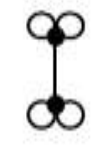

- Einperiodische Ketten:

$$
2 p: \infty \int_{0} 3 p: \oint_{0}^{\infty}
$$

- Kreisklassen: 2418
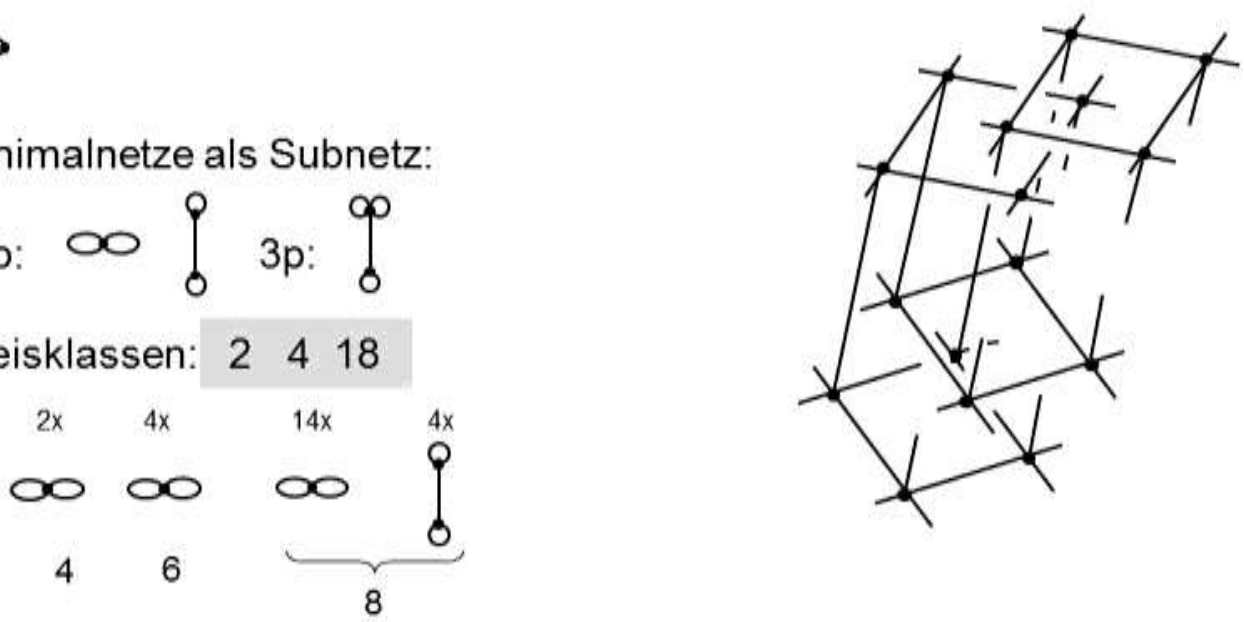

Abb. 7.6 Minimalnetz 2(5)3 


\section{$2(3,7) 1$}

Sechsecknetze werden über 2-periodische Gitternetze miteinanderverbunden.

- Einperiodische Ketten:

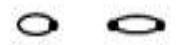

- Minimalnetze als Subnetz:

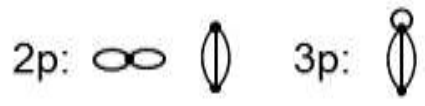

- Kreisklassen: 1973
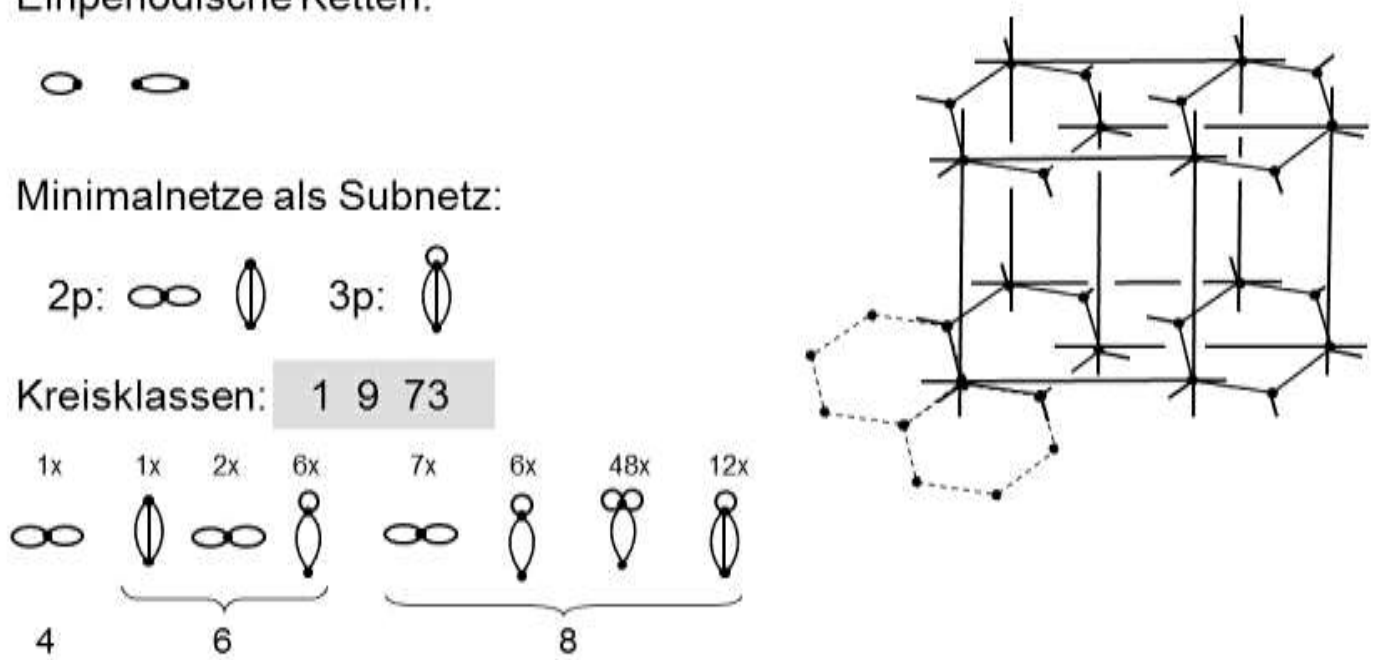

Abb. 7.7 Minimalnetz 2(3,7)1

\section{$2(3,7) 2$}

Ketten der Periodenlänge 1 verbinden 3-periodische Gitternetze über Kanten.

- Einperiodische Ketten:

o

- Minimalnetze als Subnetz:

2p: $\infty$ 3p: $\mathscr{\rho}_{0}^{\infty}$

- Kreisklassen: 322210
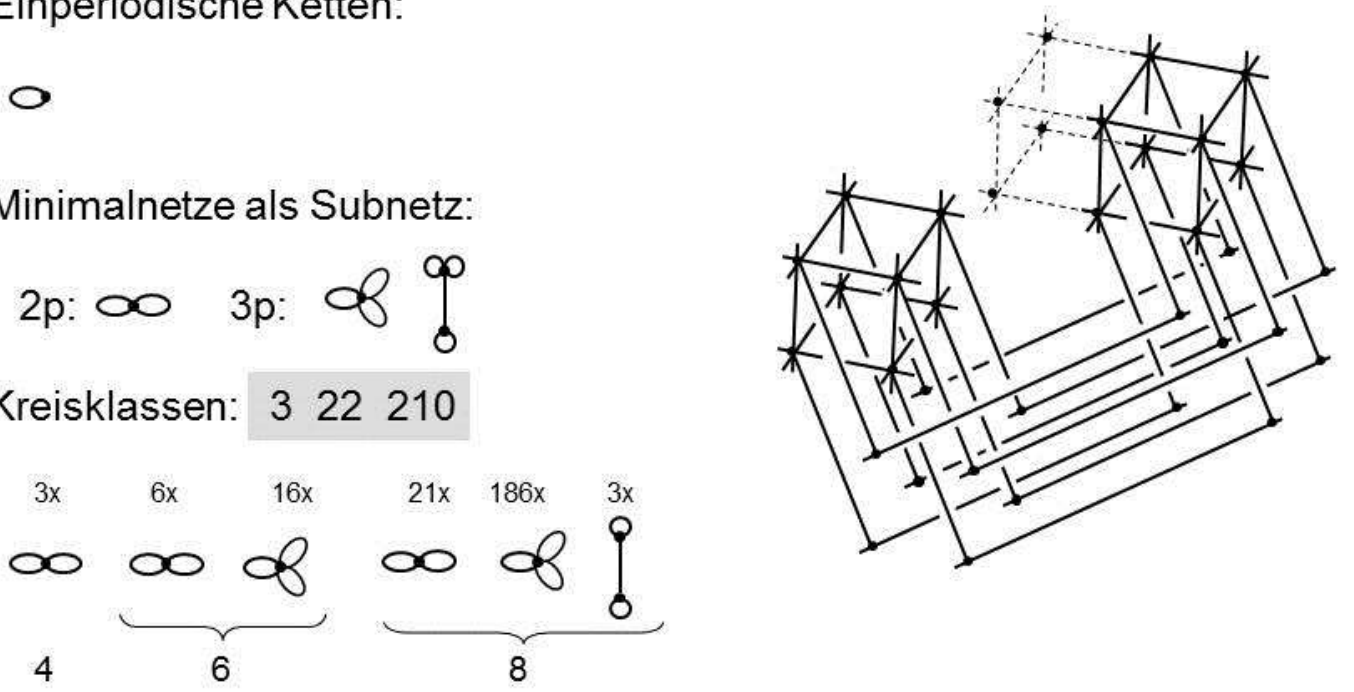

Abb. 7.8 Minimalnetz 2(3,7)2 


\section{$2(4,6) 1$}

Diamantnetze über Ketten der Periodenlänge 1 miteinanderverbunden

- Einperiodische Ketten:

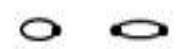

- Minimalnetze als Subnetz:

$$
\text { 2p: } 1 \mathrm{p}: \bigotimes(
$$

- Kreisklassen: 01036
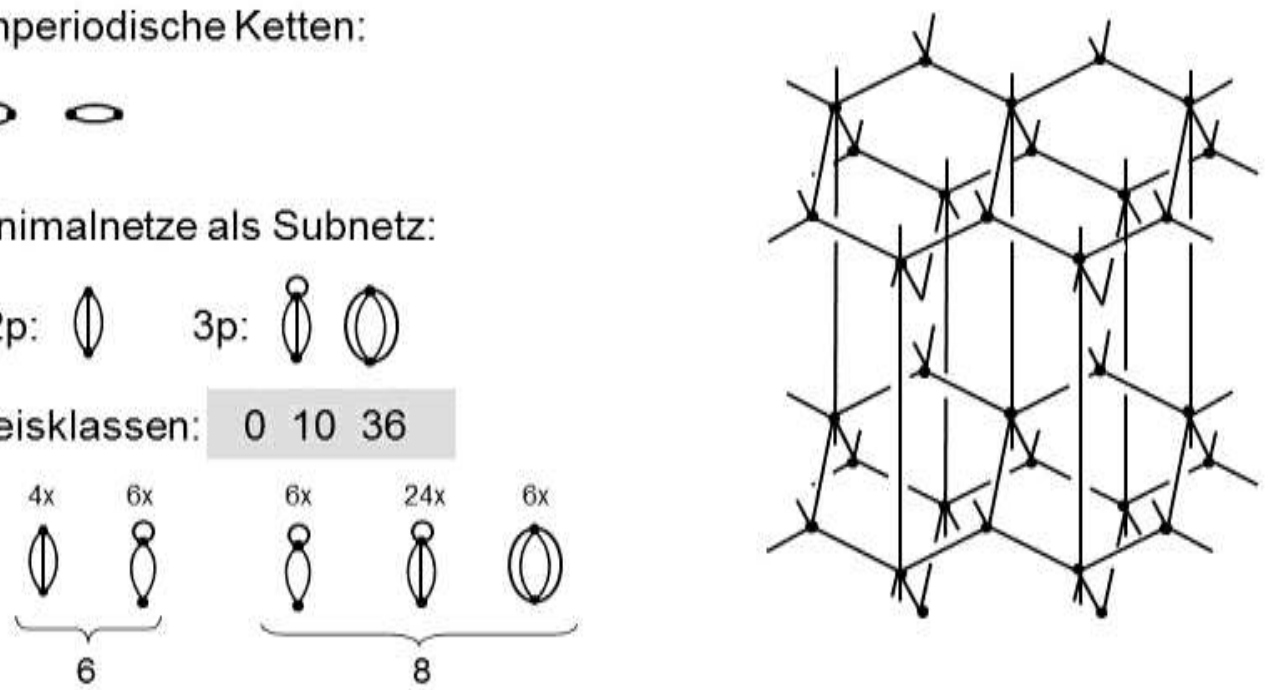

Abb.7.9 Minimalnetz 2(4,6)1

$2(4,6) 2$

Ketten der Periodenlänge 1 und 2 verbinden 2-periodische Gitternetze über Kanten.

- Einperiodische Ketten:<smiles>[Co]</smiles>

- Minimalnetze als Subnetz:

$$
\text { 2p: } \infty \text { ?p: }
$$

- Kreisklassen: 1538
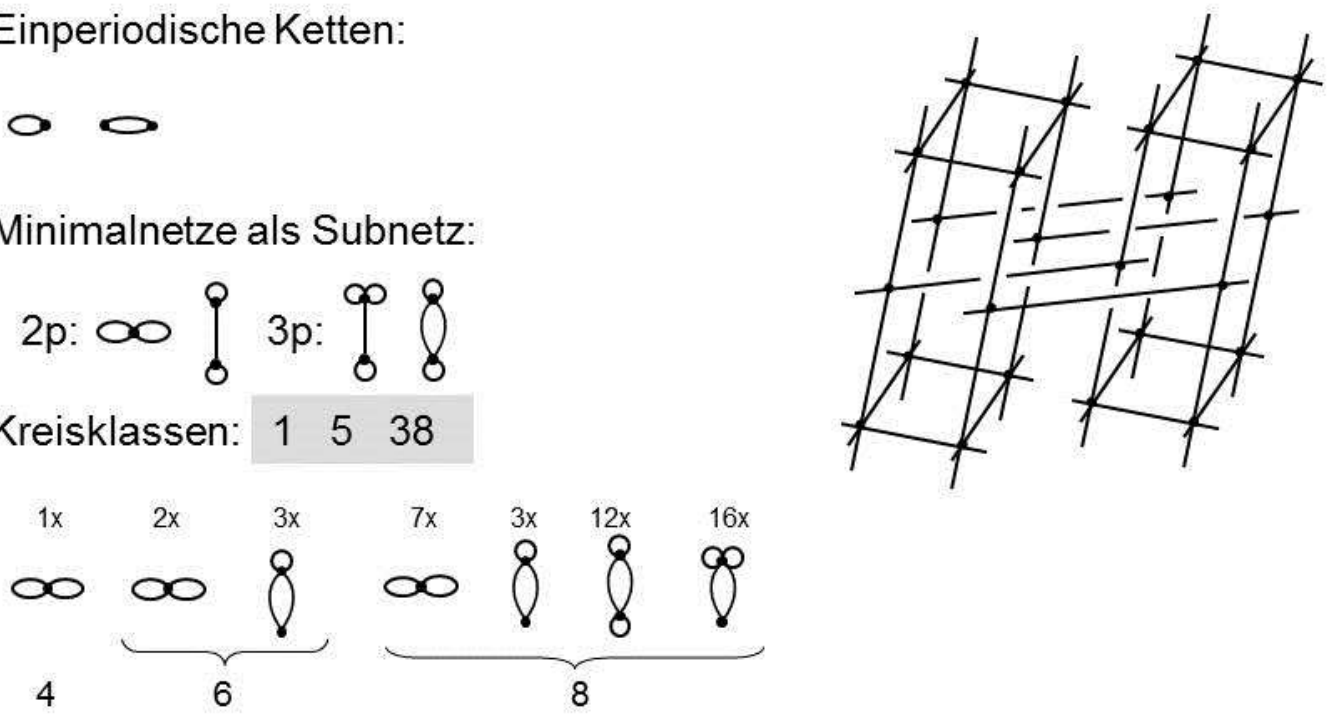

Abb. 7.10 Minimalnetz 2(4,6)2 


\section{$6(3) 1$}

1-periodische Ketten der Periodenlänge 6,

in 3 unabhängige Richtungen miteinanderverbunden.

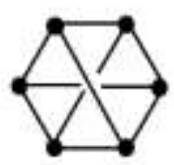

- Einperiodische Ketten:

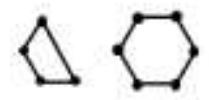

- Minimalnetze als Subnetz:

keine

- Kreisklassen: 000000618 Typen:
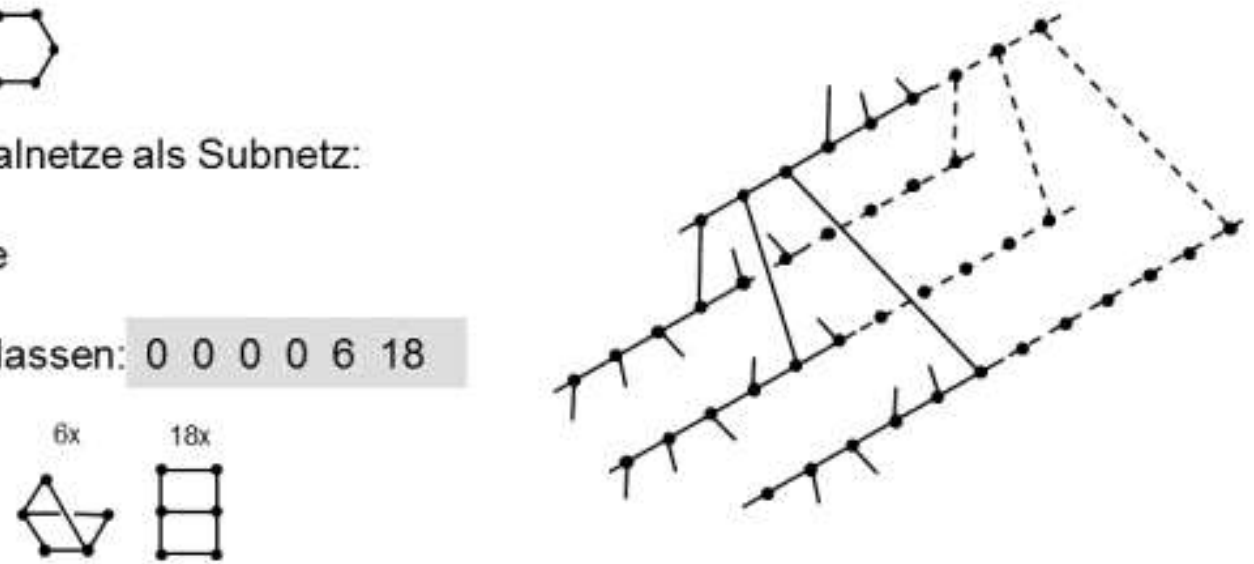

$12 \quad 14$

Abb. 7.11 Minimalnetz 6(3)1

\section{$6(3) 2$}

0-periodische Substruktur (Baum),

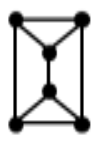

in 4 unabhängige Richtungen miteinander verbunden.

- Einperiodische Ketten:

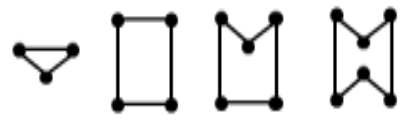

- Minimalnetze als Subnetz:

keine

- Kreisklassen: 000000615 Typen:
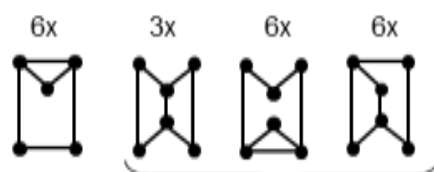

14

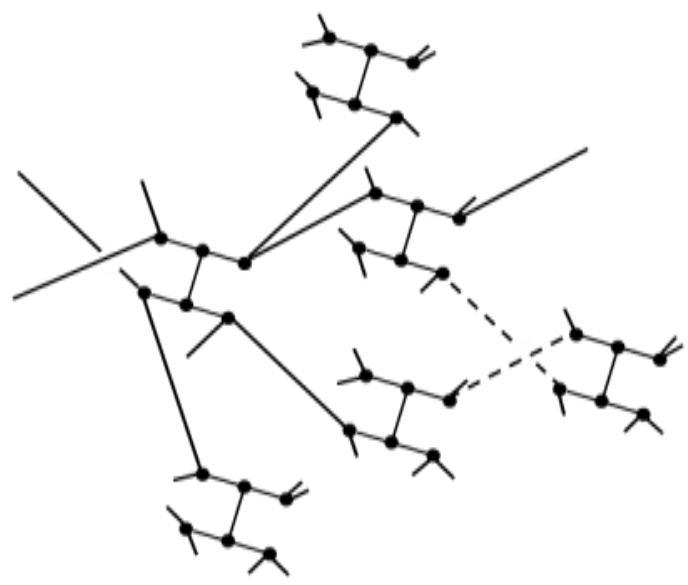

12

Abb. 7.12 Minimalnetz 6(3)2 


\section{$6(3) 3$}

Eine Kette der Periodenlänge 2 verbindet 2-periodische Strukturen aus Kreisen der Länge 10.

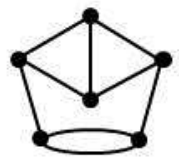

- Einperiodische Ketten:

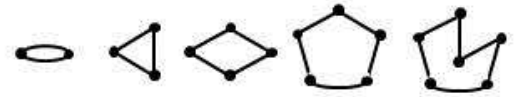

- Minimalnetze als Subnetz:

keine

- Kreisklassen: $0 \begin{array}{lllll}0 & 0 & 1 & 2 & 14\end{array}$ Typen:
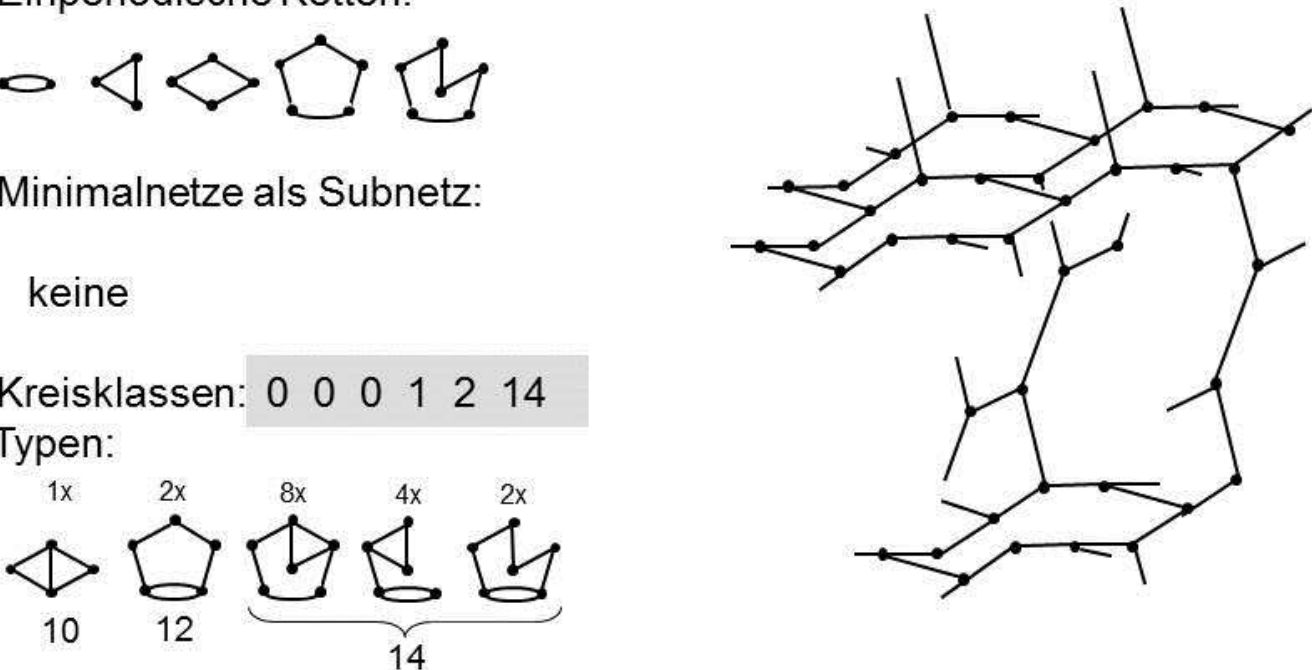

Abb. 7.13 Minimalnetz 6(3)3

\section{$6(3) 4$}

1-periodische Ketten der Periodenlänge 6,

in 3 unabhängige Richtungen miteinander verbunden.

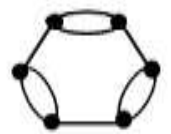

- Einperiodische Ketten:

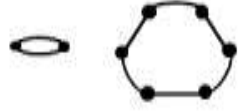

- Minimalnetze als Subnetz:

keine

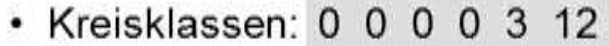
Typen:
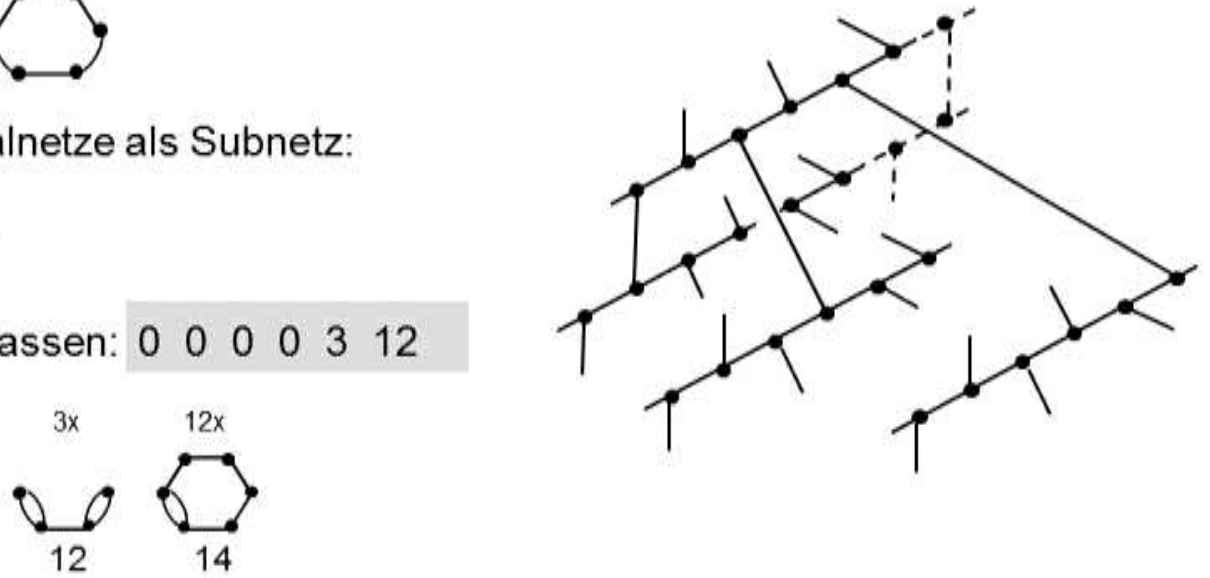

Abb. 7.14 Minimalnetz 6(3)4 


\section{Minimalnetz 6(3)1 - Penrose-Muster zur Konstruktion der 12er-Kreise}

Der $\mathrm{K}_{3,3}$ (Abb. 8.1) ist ein paarer Graph - denn seine Knotenmenge lässt sich in zwei Teilmengen zerlegen, sodass jede Kante einen Knoten der einen Menge mit einem der anderen verbindet [Harary, 1969]. Die dreiperiodischen Netze des $K_{3,3}$ wurden von [Radke, 1985] und [Beukemann, Klee, 1988] untersucht. Es gibt ein vierperiodisches Netz des $K_{3,3}$ : das Minimalnetz 6(3)1. Sämtliche Knoten so wie auch sämtliche Kanten dieses Netzes sind topologisch gleichwertig.

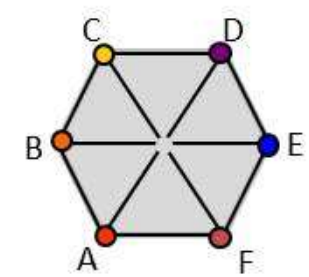

Abb. 8.1 Der Graph $\mathbf{K}_{3,3}$. Die sechs Knoten, die im dazugehörenden Netz den sechs Knotenklassen entsprechen, werden in dieser und in den anderen Abbildungen dieses Kapitels durch unterschiedliche Farben gekennzeichnet.

Das Minimalnetz 6(3)1 hat folgendes Kreisklassenspektrum:

\begin{tabular}{lcccccc} 
Kreislänge & 4 & 6 & 8 & 10 & 12 & 14 \\
\hdashline$-n_{1}$ & 0 & 0 & 0 & 0 & 6 & 18
\end{tabular}

Die Untersuchung der Kreise dieses Netzes zeigt, dass die Kreise der sechs Kreisklassen der Länge 12 alle topologisch gleichwertig sind, und dass an einem 12er-Kreis die Knoten fünf verschiedener Knotenklassen beteiligt sind.

Betrachtet man einen beliebigen Kreis der Länge 12, so wechseln sich Knoten von Knotenklassen, die dreimal im Kreis vorkommen, mit solchen, die zweimal darin vorkommen, ab.

Im Minimalnetz 6(3)1 sind all diese 6 Kreisklassen in Bezug auf die Folgen ihrer Knoten, und damit auch ihrer Kanten, nach dem gleichen Schema aufgebaut:

- I - II - III - VI - I - V - III - II - I - VI - III - V -

In einem 12er-Kreis sind jeweils Knoten von 5 der 6 Knotenklassen beteiligt. Die Knoten zweier Klassen (I und III) kommen je dreimal vor (Abstand zwischen 2 Knoten derselben Klasse: 4 Kantenlängen). Die Knoten der drei anderen Klassen kommen je zweimal vor, und die Knoten einer solchen Klasse liegen im Kreis gegenüber (Abstand: 6 Kantenlängen). 
Es hat sich gezeigt, dass Kreise des 4-periodischen Minimalnetz 6(3)1 und einzelne Polygone eines Penrose-Musters (Abb. 8.2) zur Deckung und somit beide Strukturen in einen gewissen topologischen Zusammenhang gebracht werden können. Es gibt sicher andere höherperiodische (>3) Netze, für die dies ebenfalls gilt.

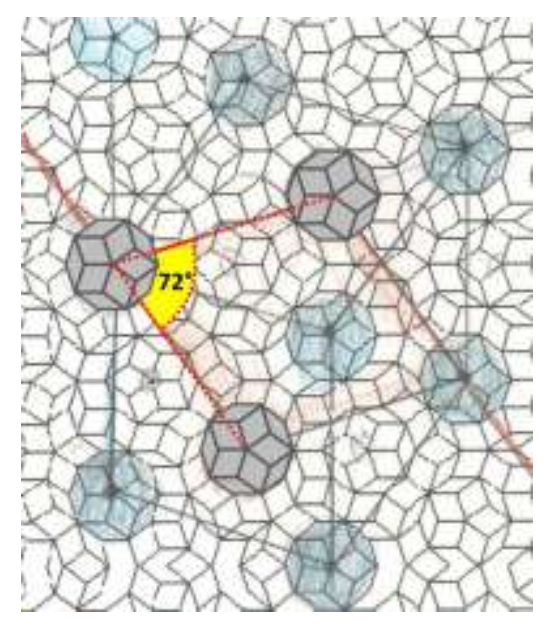

Abb. 8.2. Penrose-Muster. Aperiodisch angeordnete unterschiedlich geformte Polygone füllen die Fläche vollständig und lückenlos aus. Erkennbar sind in dieser Abbildung lokale (im grauen Bereich) bzw. langreichweitige (zwei graue Bereiche werden durch Drehung um den gelb eingezeichneten Winkel von $72^{\circ}$ zur Deckung gebracht) 5-zählige Symmetrieoperationen, die in 3-periodischen Kristallstrukturen nicht vorkommen können.

Ein Vertreter einer Kreisklasse der Länge 12 des Minimalnetzes 6(3)1 wird nun so in ein Penrose-Muster eingebettet, dass seine Knoten in diesem Muster an den Ecken oder den Kantenmitten eines Polygons des Penrose-Musters liegen (Kreis Nr. 1 in Abb. 8.3).

Ausgehend von diesem Kreis werden die Vertreter der anderen fünf Kreisklassen dieser Länge so konstruiert, dass sie an diesen Kreis angefügt werden können (Abb. 8.3).

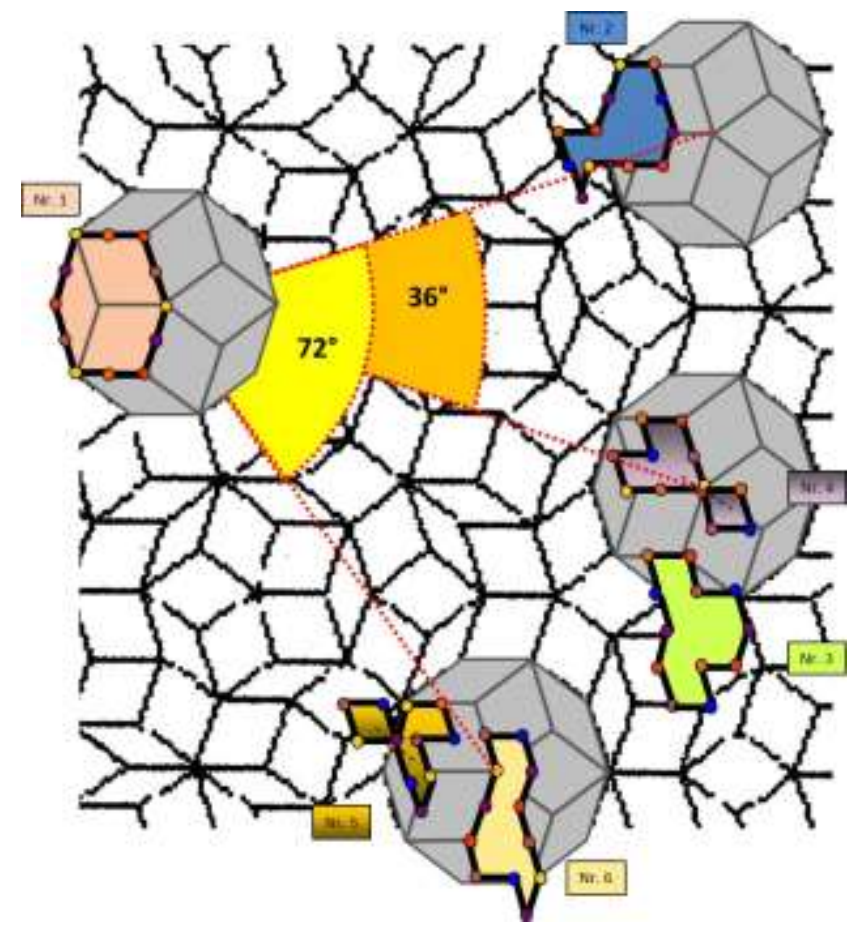

Abb. 8.3 Einbettung von Kreisen der Länge 12 ins Penrose-Muster.

Nr. 1 bis Nr.6: Vertreter von Kreisen der sechs Kreisklassen mit Kreisen der Länge 12. 
Für jede Kreisklasse der Kreislänge 12 ist in Abb. 8.4 die Kantenfolge im Quotientengraphen und eine 2-periodische Substruktur gezeigt.

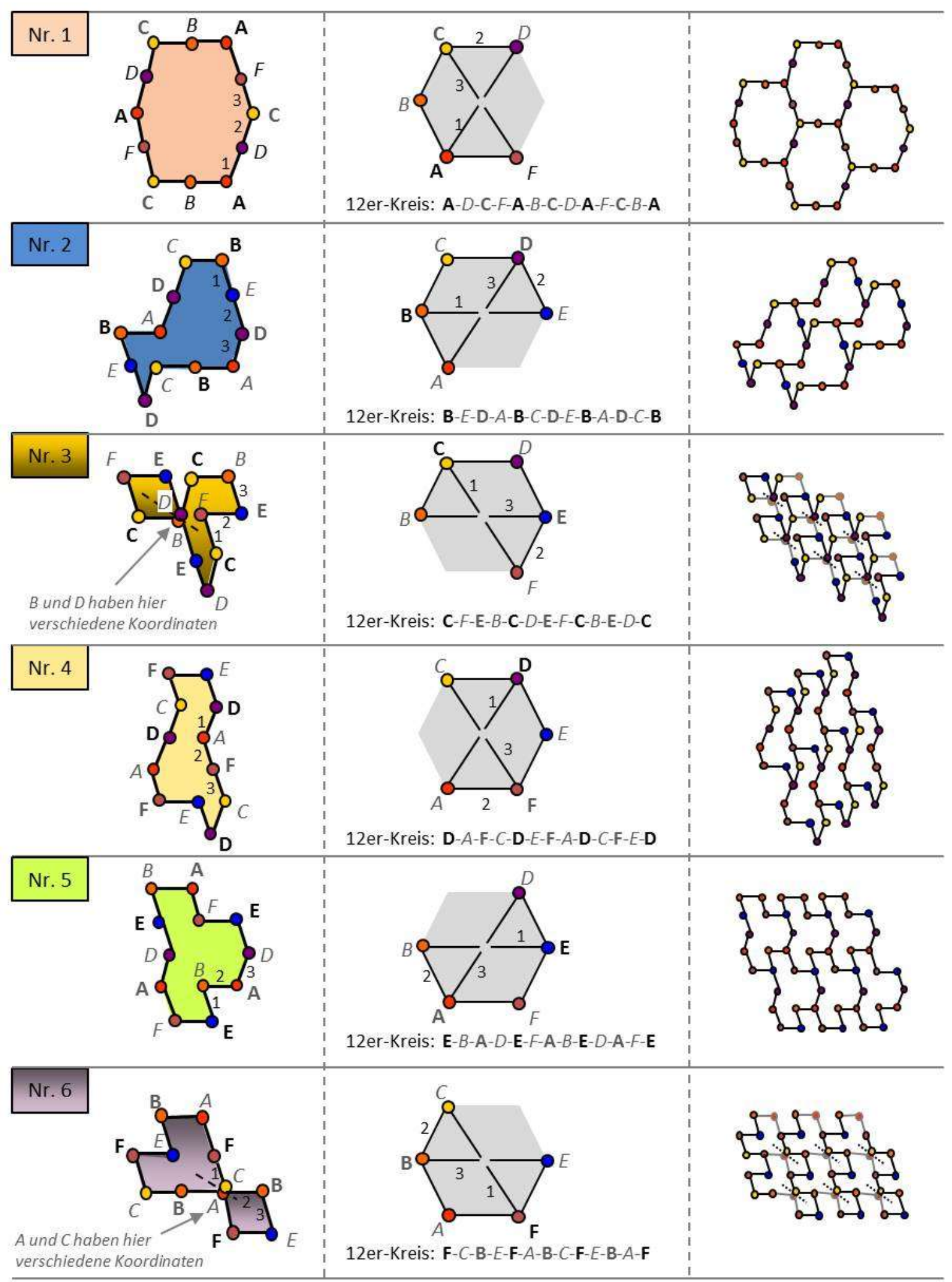

Abb. 8.4 Kantenfolgen in den 12er-Kreisen des Minimalnetzes 6(3)1. 12er-Kreise einer Kreisklasse dieses Netzes lassen sich lückenlos zu einem 2-periodischen Muster zusammenfügen. Betrachtet man in diesem Netz alle 12er-Kreise einer Kreisklasse, so bilden sie eine Schar aus solchen 2-periodischen Mustern. Die Kantenfolgen sämtlicher Kreise folgen dem Schema:

- I - II - III - VI - I - V - III - II - I - VI - III - V - 
Die 2-periodischen Substrukturen aus Kreisen einer Kreisklasse sind über Kreise anderer Kreisklassen miteinander verbunden (Abb. 8.5).
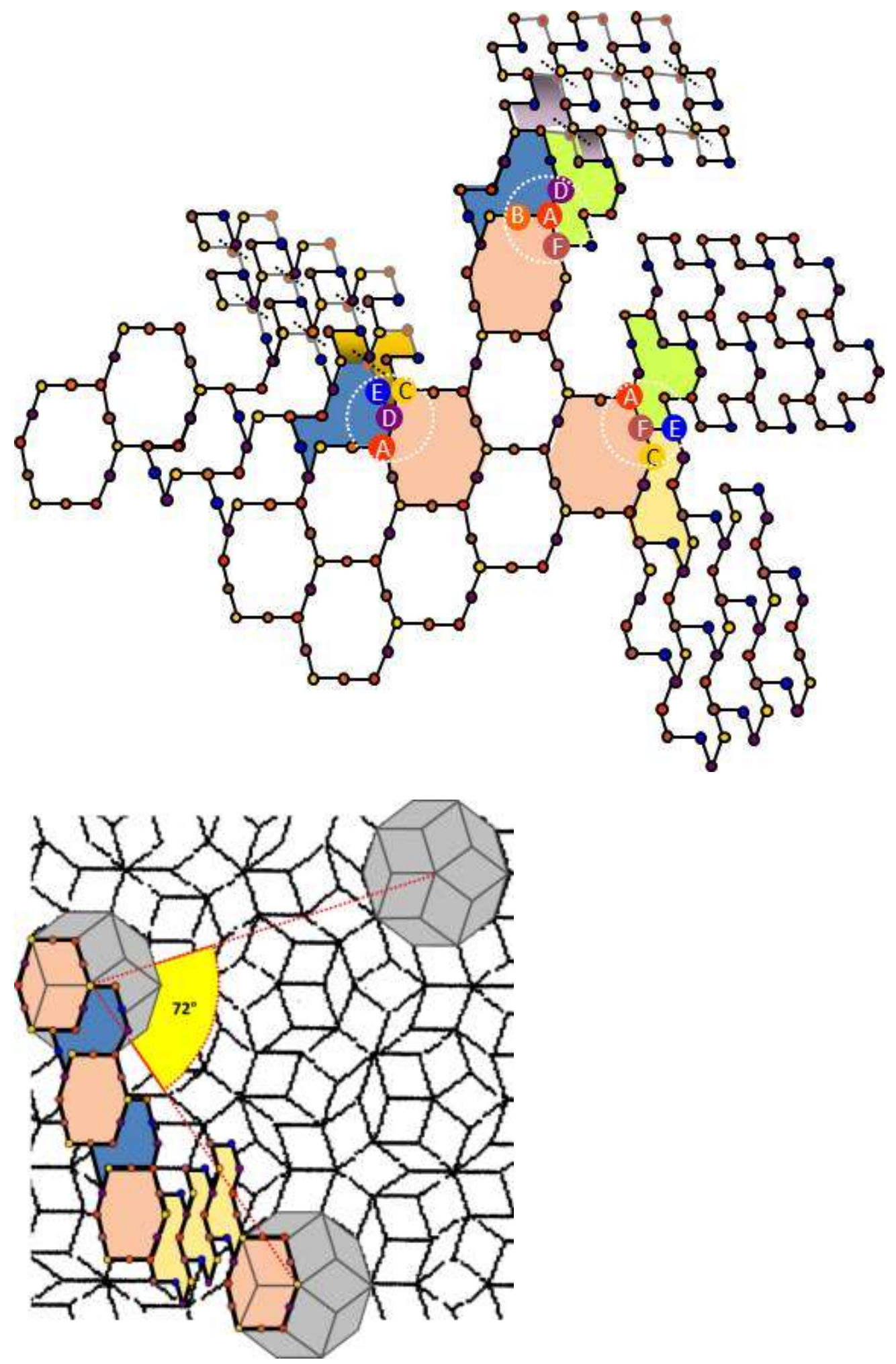

Abb. 8.5 Unterschiedliche 2-periodische Substrukturen. Unten: Zwei Kreise der Kreisklasse Nr. 1 (vgl. ihre Position mit Abb. 8.2) sind über Kreise anderer Kreisklassen miteinander verbunden. 
Betrachtet man einen einzelnen Kreis einer Substruktur (z. B. Nr. 1 aus Abb. 8.4), so sind andere zweiperiodische Substrukturen (z. B. Nr. 2) über Knoten, die maximal vier unterschiedlichen Knotenklassen angehören, miteinander verbunden.

Zwei Fälle sind zu unterscheiden:

- Fall 1

Ein Knoten, der zweimal im Kreis vorkommt, ist in den Kreisen einer anderen Kreisklasse nicht enthalten.

Beispiel (Abb. 8.6, links):

Knoten F, zweimal in Kreis „Nr. 1“, kommt in Kreis Nr. 2 nicht vor.

Ein einzelner Kreis „Nr. 1“ ist über die Knoten A, D, C, B mit zwei Substrukturen aus

Kreisen „Nr. 2“verbunden

- Fall 2

Ein Knoten, der dreimal im Kreis vorkommt, ist in den Kreisen einer anderen Kreisklasse nicht enthalten.

Beispiel (Abb. 8.6, rechts):

Knoten C, dreimal in Kreis „Nr. 1“, kommt in Kreis Nr. 5 nicht vor.

Ein einzelner Kreis „Nr. 1“ ist mit drei Substrukturen aus Kreisen „Nr. 5“ über jeweils drei Knoten (über B, A, F; über D, A, F; über D, B, A) verbunden
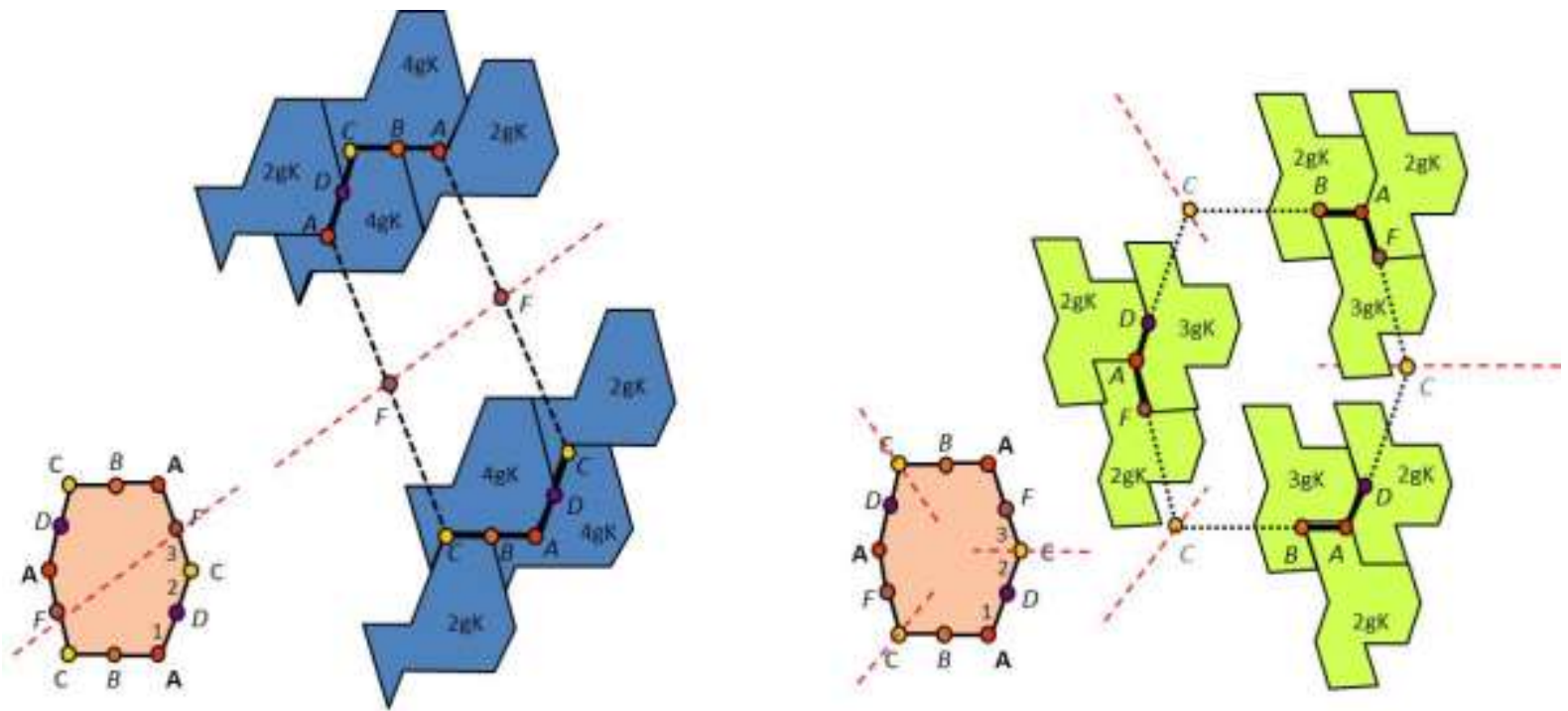

Abb. 8.6 Ein Kreis der Substruktur Nr.1 verbindet Substrukturen (Nr.2 bzw. Nr.5).

Die Anzahl der Knoten, die der betrachtete Kreis mit den einzelnen Kreisen der Substrukturen (Nr.2 bzw. Nr.5) gemeinsam hat, ist unterschiedlich (gK bedeutet gemeinsame Knoten). 
Nun wird die Topologie des Minimalnetzes 6(3)1 aus einem anderen Blickwinkel betrachtet. Die sechs unterschiedlichen Knoten hängen über eine Baumstruktur zusammen. Die einzelnen Baumstrukturen sind untereinander über vier indizierte Kanten verknüpft (Abb. 8.7)

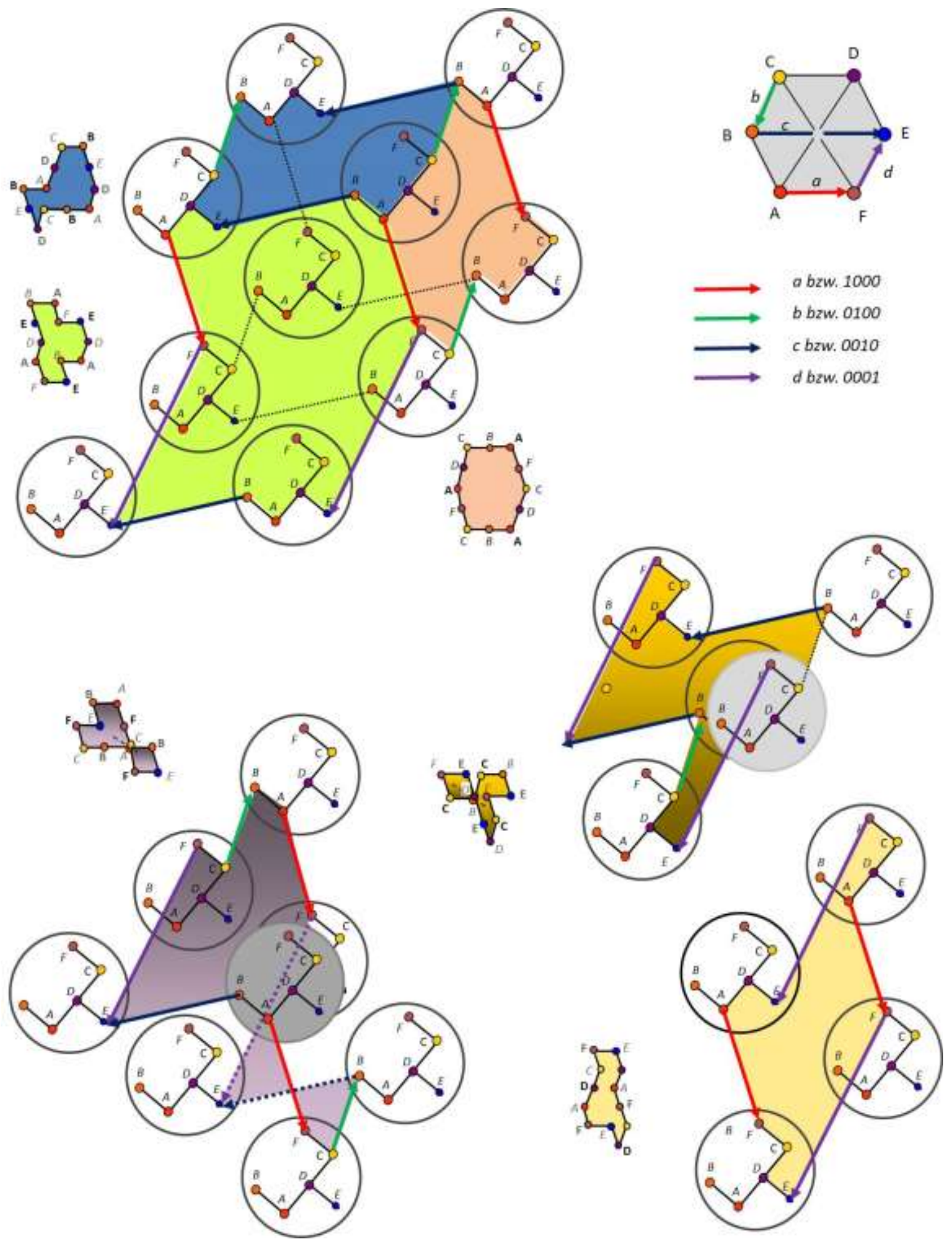

Abb. 8.7 Andere Darstellung des Minimalnetzes 6(3)1 (Teilbereiche). Oben rechts: indizierter Quotientengraph, unterschiedliche Farben für die indizierten Kanten. Links daneben und darunter: Ausschnitte des Netzes mit Darstellung der einzelnen (topologischen) Kreise Nr. 1 bis Nr. 6 aus Abb. 8.4. Die einzelnen Baumstrukturen (dritter Graph von oben im Kästchen $n=6$ in Abb. 3.3) sind durch geometrische Kreise eingerahmt. 
Die einzelnen Baumstrukturen sind untereinander verknüpft wie die Gitterpunkte eines 4-dimensionalen Gitters (Abb. 8.8).

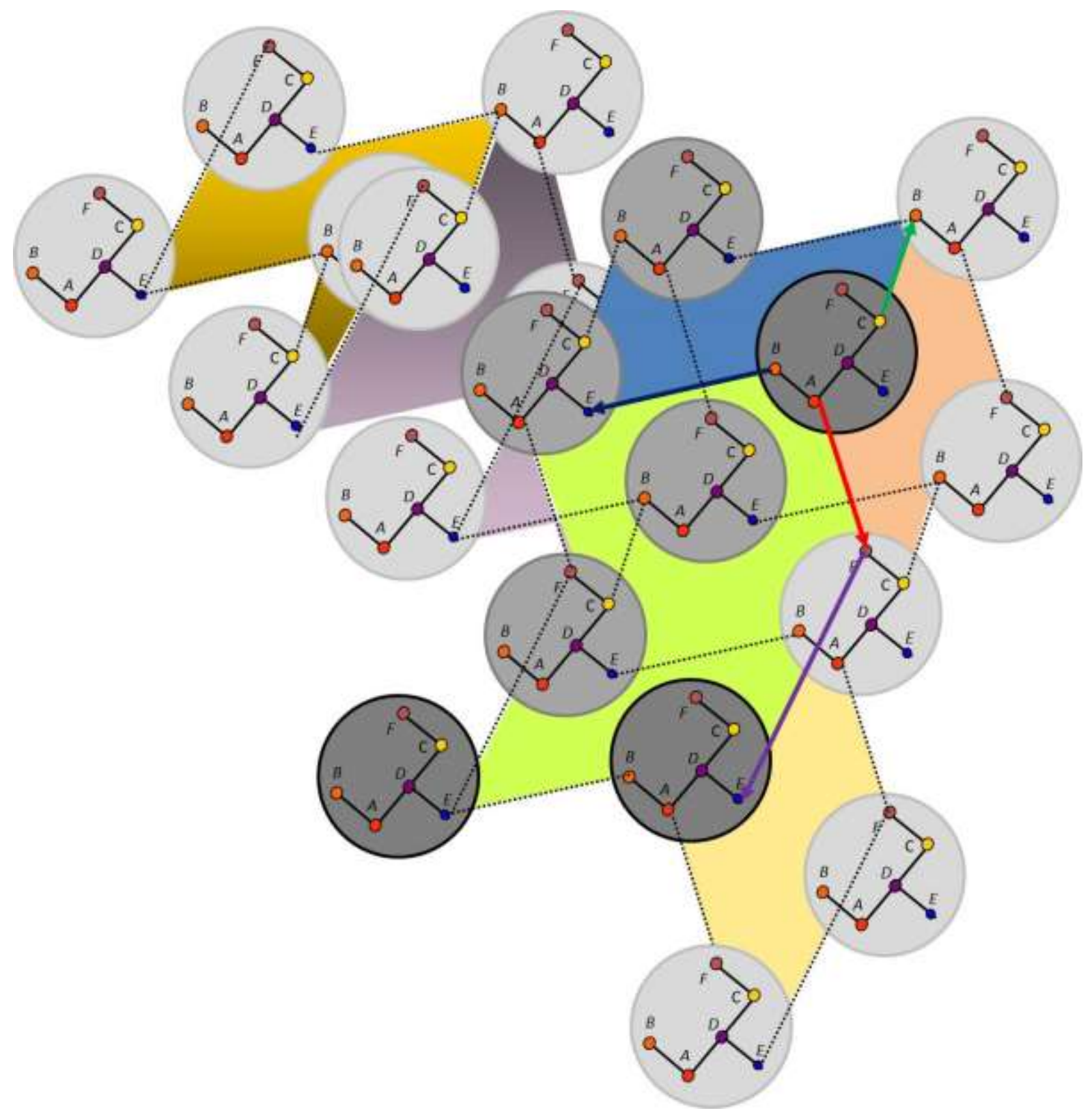

Abb. 8.8 Andere Darstellung des Minimalnetzes 6(3)1 (Gesamtsicht). Teilbereiche sind in Abb. 8.7 dargestellt. 


\section{Einbettung des Minimalnetzes 3(4)1 mit symmetrischer Indizierung}

Auch beim Minimalnetz 3(4)1 sind sowohl die Knoten, als auch die Kanten topologisch gleichwertig. Um eine möglichst symmetrische Einbettung dieses Netzes in den vierdimensionalen Raum zu erzielen, wird in diesem Abschnitt ein besonderes Verfahren zur Indizierung der Kanten verwendet.

Um 6 Kanten ,gleichwertig“ zu indizieren, bieten sich jeweils die Paare der Basisvektoren der sechs zweidimensionalen Unterräume an:

$$
\mathrm{ab}=1100, \mathrm{~cd}=0011, \mathrm{ac}=1010, \mathrm{bd}=0101, \mathrm{ad}=1001, \mathrm{bc}=0110
$$

Eine mögliche Indizierung ist in Abb. 9.1 gezeigt:

- Betrachtet man eine Doppelkante, so besteht die zweite Kante aus den Basisvektoren, die in der ersten nicht vorkommen. Jede Kante verläuft entlang der Diagonale der Fläche, die durch ihre zwei Basisvektoren aufgespannt ist.

- Der Pfeil auf der Kante gibt hier jeweils die Richtung beider Basisvektoren an.

Der Pfeil auf der blau eingefärbten Kante von A nach B bedeutet, dass man von A über die Vektorsumme $(\mathrm{a}+\mathrm{b})$ nach $\mathrm{B}$ gelangt. Von B nach A kommt man dann durch den entgegengesetzen Vekor (-a-b).

- Betrachtet man den gesamten Quotientengraphen, so sind die Richtungen der Pfeile im Uhrzeigersinn gesetzt.

Neben der Indizierung des Quotientengraphen des Minimalnetzes 3(4)1 zeigt Abb. 9.1 auch die Nachbarschaftsbeziehungen der Knoten A, B, C.im Netz.
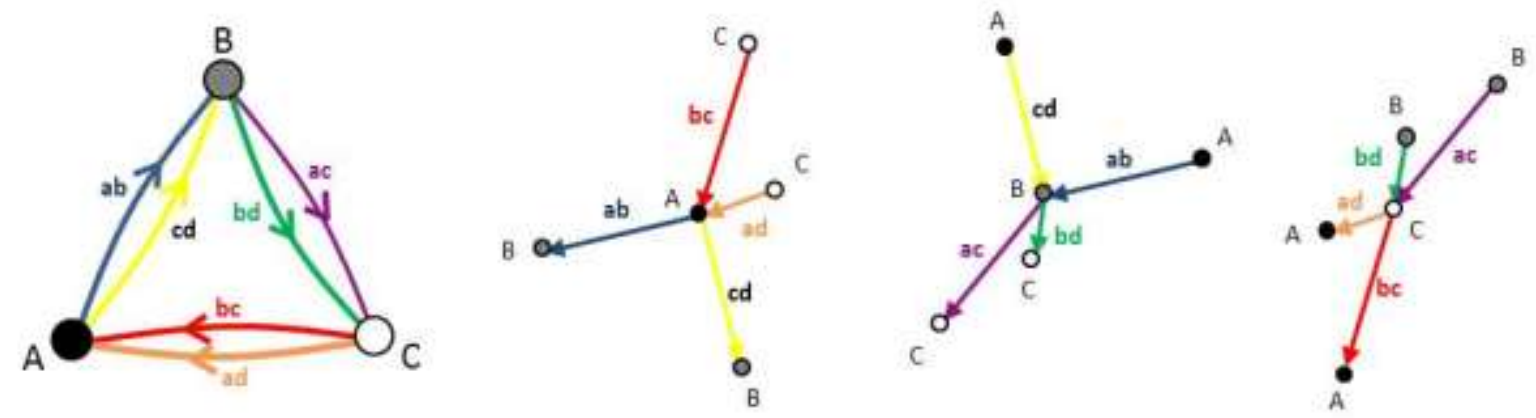

Abb. 9.1 Minimalnetz 3(4)1. Links: Quotientengraph mit symmetrisch indizierten Kanten. Rechts: die einzelnen Knoten (A, B, C) und ihre Nachbarschaftsbeziehungen. 
Die vier Basisvektoren a, b, c, d, die den vierdimensionalen Raum aufspannen, sind in Abb. 9.2 zusammen mit den sechs Flächendiagonalen gezeigt. Zur Verdeutlichung der Position der sechs Flächen wurden ihnen farbige Dreiecke zugeordnet.
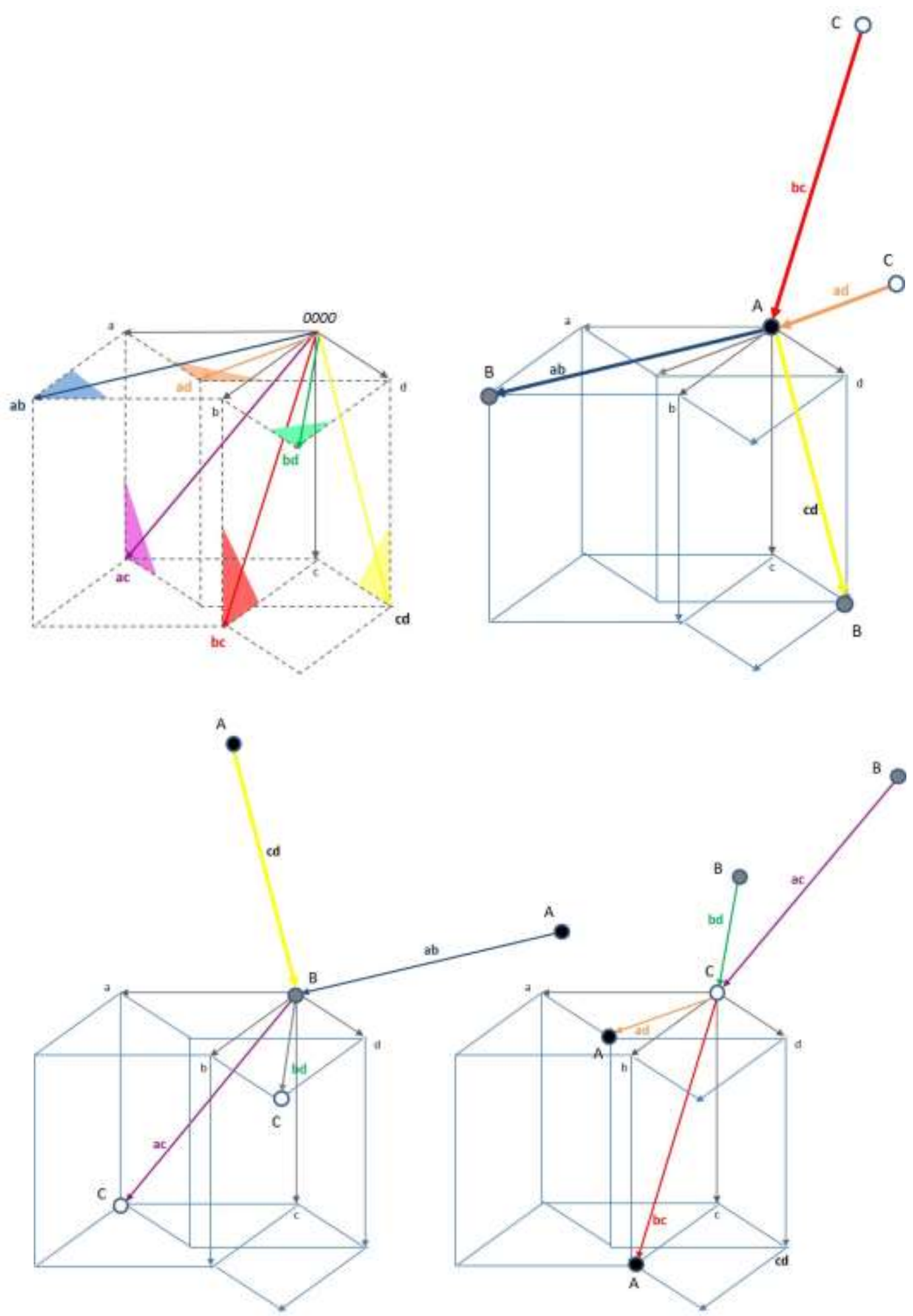

Abb. 9.2 Die vier Basisvektoren a, b, c, d und die sechs Flächendiagonalen. Oben links:

Basisvektoren, Flächendiagonalen. Die Nachbarschaftsbeziehungen von Knoten A (oben rechts), von Knoten B (unten links), und von Knoten C (unten rechts). 
Im Minimalnetz 3(4)1 gibt es 15 Kreisklassen mit Kreisen der Länge 8. Sie können in zwei Typen eingeteilt werden, die sich bezüglich ihrer 2-periodischen Substruktur unterscheiden. Drei Kreisklassen gehören Typ 1 an, die zwölf anderen sind vom Typ 2 (Abb. 9.3).

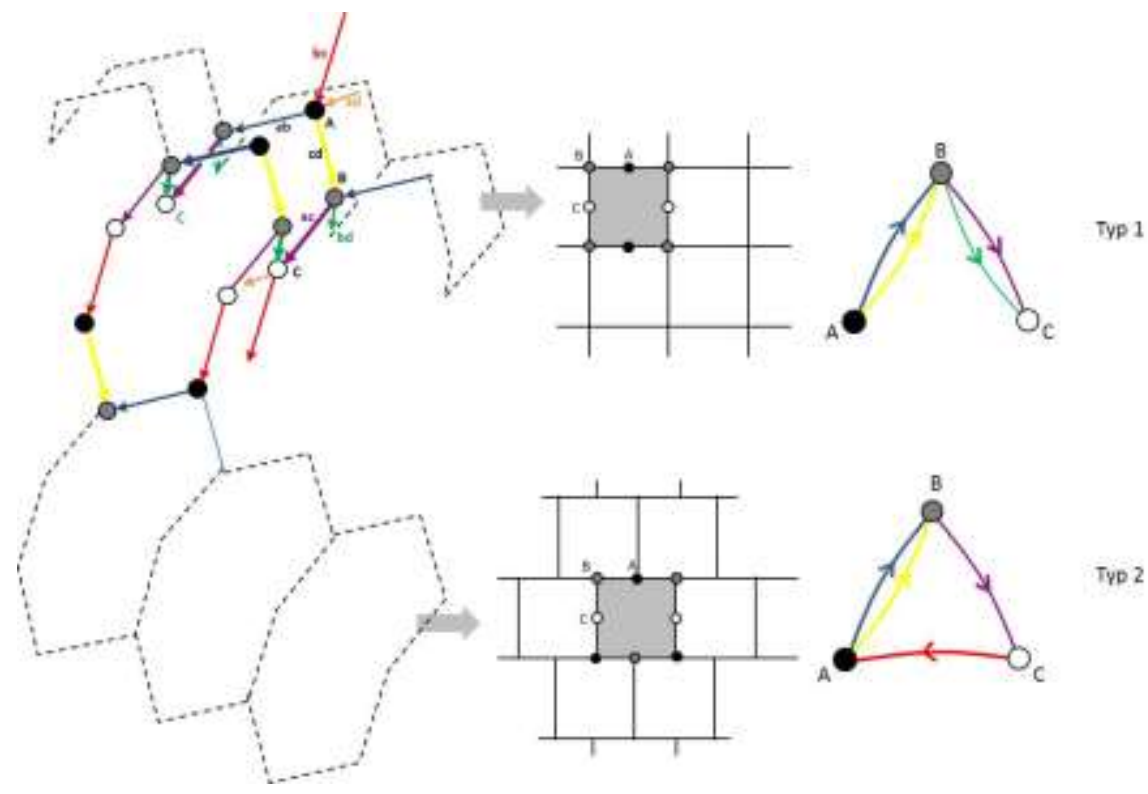

Abb. 9.3 Zwei Typen von 8er-Kreisen. Die unterschiedlichen 2-periodischen Substrukturen entsprechen unterschiedlichen Teilstrukturen (vgl. Abb. 3.14) des Quotientengraphen.

Die Vertreter der 15 Kreisklassen mit Kreisen der Länge 8 sind in Abb. 9.4 gezeigt. Die Farbgestaltung dient hier zur besseren Unterscheidung der Kreise innerhalb einer Skizze. Auch die Projektion der Netzausschnitte auf die Ebene wurde für diesen Zweck geändert.

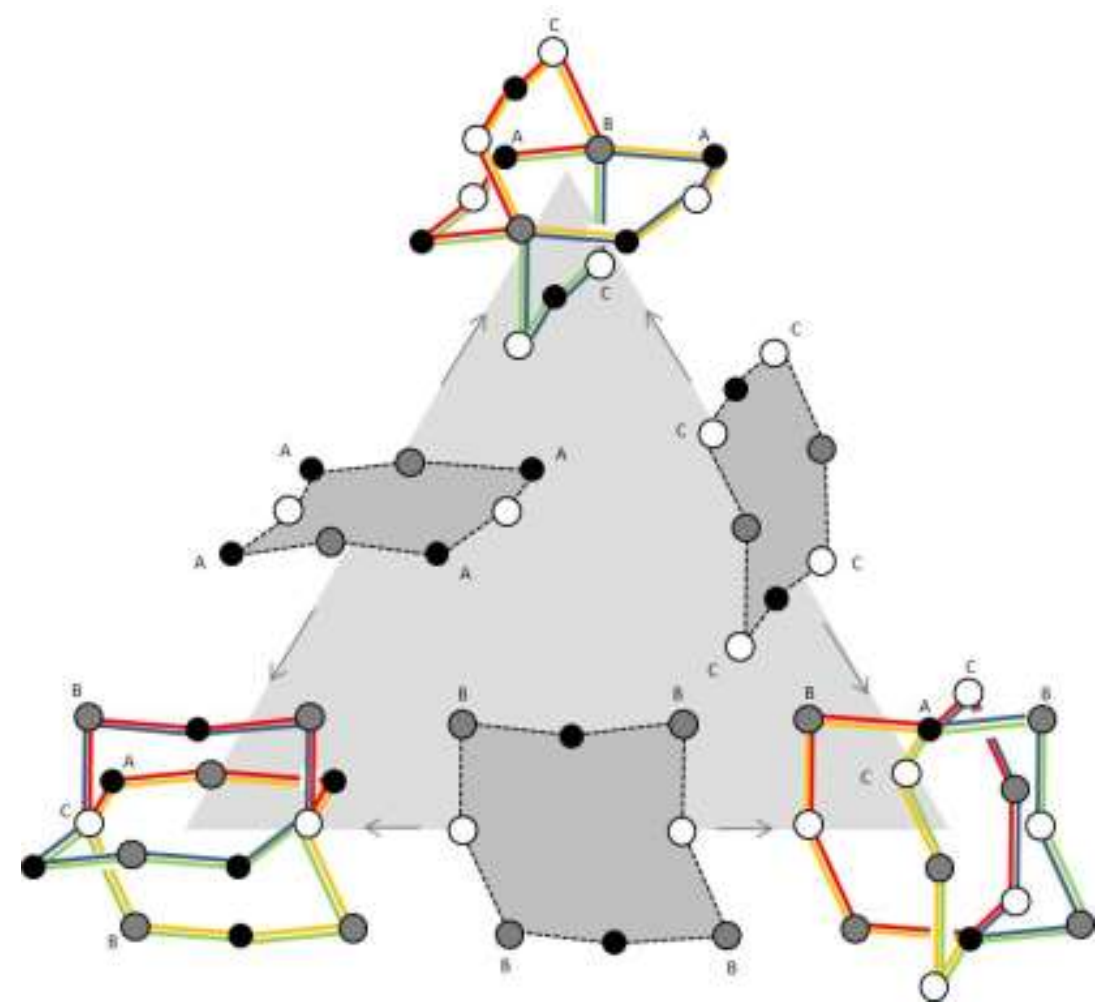

Abb. 9.4 Die 15 Kreisklassen mit Kreisen der Länge 8 des Minimalnetzes 3(4)1. Unterschiedliche Farben innerhalb der drei farbigen Skizzen in dieser Abbildung dienen zur Unterscheidung der in der jeweiligen Skizze gezeigten vier Kreise der Länge 8. 
Im Folgenden werden die Abstände zwischen den einzelnen Knoten eines Kreises bestimmt.

Die vier Basisvektoren a, b, c, d sind linear unabhängig und bilden ein hyperkubisches Gitter. Sie stehen also senkrecht aufeinander. Somit stehen die Vektoren c und d sowohl senkrecht zu a, als auch senkrecht zu b, und damit auch senkrecht zu allen Vektoren, die in der von den Vektoren a und $b$ aufgespannten Ebene liegen. Der Betrag der Länge eines jeden Basisvektors wird auf 1 festgelegt:

$$
|\mathrm{a}|=|\mathrm{b}|=|\mathrm{c}|=|\mathrm{d}|=1
$$

Nach dem Satz von Pythagoras haben die einzelnen Flächendiagonalen eine Länge von $2^{1 / 2}$.

$$
|\mathrm{ab}|=|\mathrm{ac}|=|\mathrm{ad}|=|\mathrm{bc}|=|\mathrm{bd}|=|\mathrm{cd}|=2^{1 / 2}
$$

Da die Kanten durch die oben genannten Flächendiagonalen definiert sind, haben alle Kanten die Länge „Wurzel aus 2“. Die Abstände zu den anderen Knoten lassen sich mit dem Satz von Pythagoras (auf vier Dimensionen verallgemeinert, siehe Kap. 1) berechnen.

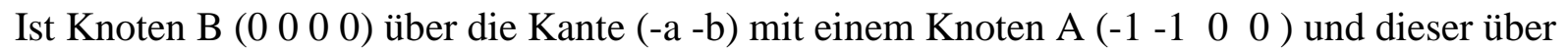
eine weitere Kante (c d) mit einem anderen Knoten B verbunden. So lassen sich seine Koordi-

\begin{tabular}{|c|c|c|c|}
\hline B & 0 & 0 & 0 \\
\hline$(-a-b)$ & -1 & -1 & 0 \\
\hline$(\mathrm{c} d)$ & 0 & 0 & 1 \\
\hline
\end{tabular}
naten durch Vektoraddition wie folgt bestimmen:

Der Abstand zwischen den beiden Knoten B( $\left.\begin{array}{lllll}0 & 0 & 0 & 0\end{array}\right)$ und B(-1 $\left.\begin{array}{llll}-1 & 1 & 1\end{array}\right)$ beträgt: $\left[(-1)^{2}+(-1)^{2}+1^{2}+1^{2}\right]^{1 / 2}=4^{1 / 2}=2$.

Einige Abstände zweier zu einem 8er-Kreis gehörender Knoten werden in Abb. 9.5 gezeigt.
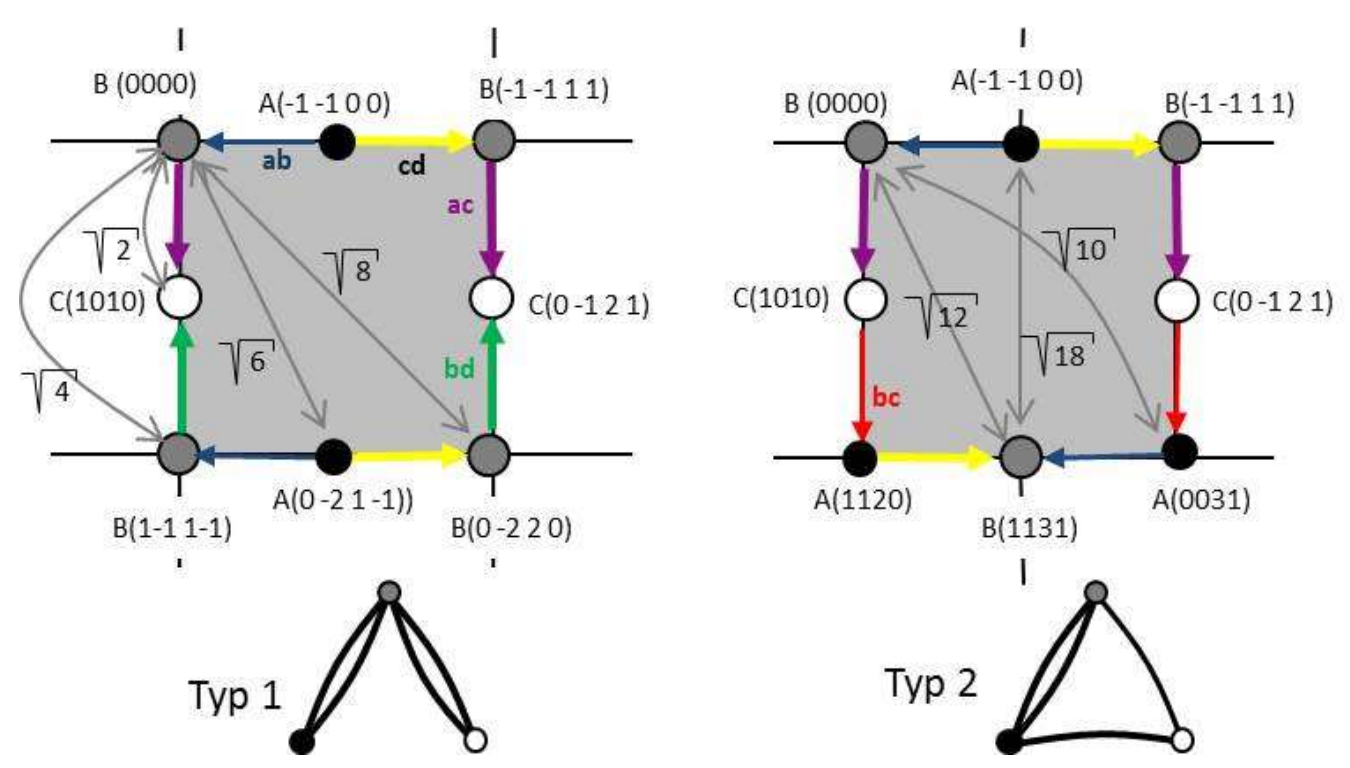

Abb. 9.5 Einige Abstände in 8er-Kreisen des Minimalnetzes 3(4)1. Die zwei Typen der 8er-Kreise sind in Abb. 9.3 gezeigt. Berechnung der Abstände im Text über dieser Abbildung. 
In Abb. 9.6 haben die einzelnen Kreise, die konzentrisch um $\mathrm{B}(0000)$ angeordnet sind, die in der kleinen Skizze (oben) angegebenen Radien. Die ablesbaren Entfernungen (unten) beziehen sich auf den Punkt B(0000), der im Ursprung eines vierdimensionalen Koordinatensystems liegt.
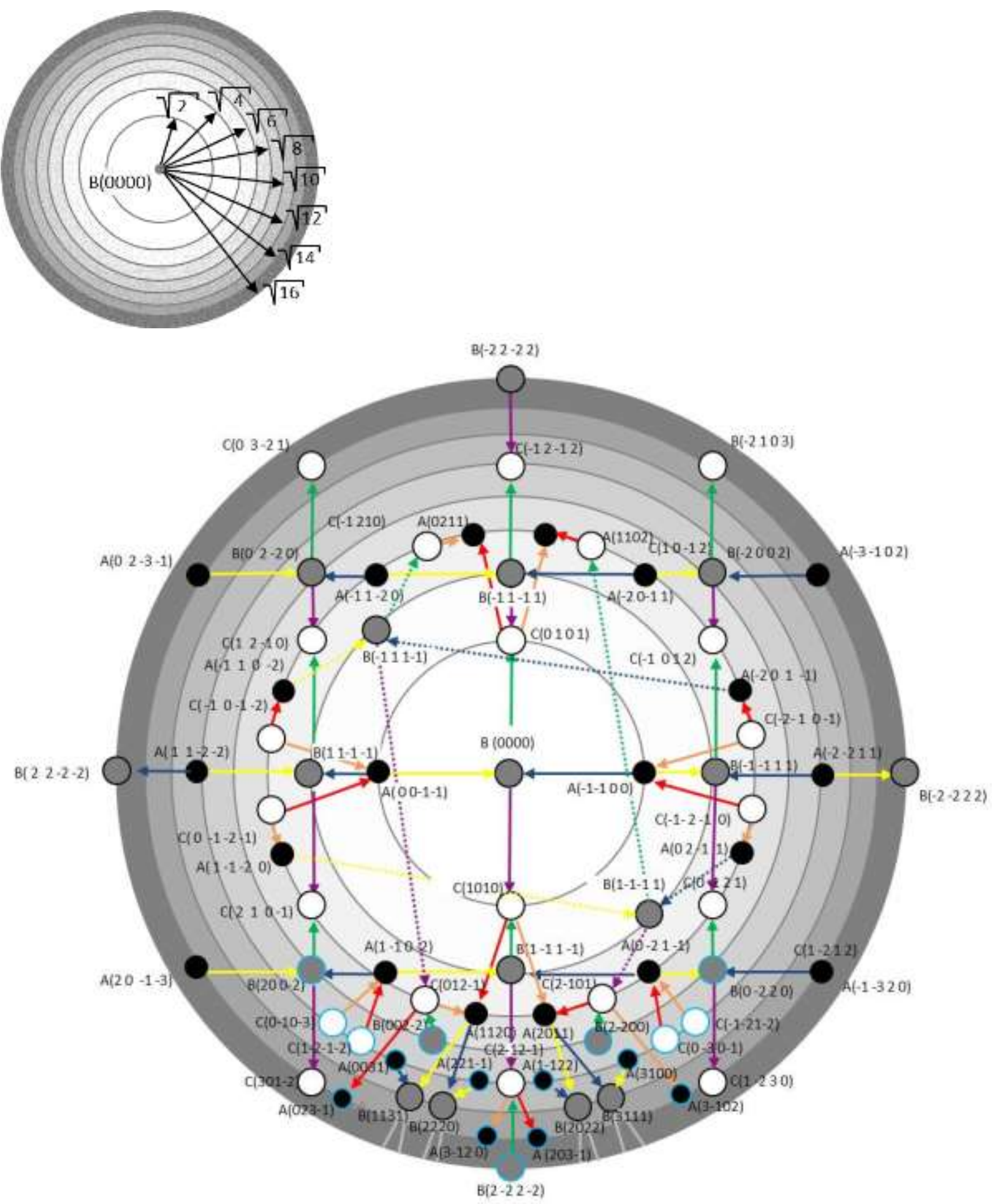

Abb. 9.6 Abstände zwischen B(0000) und anderen Knoten des Minimalnetzes 3(4)1. Knoten, die

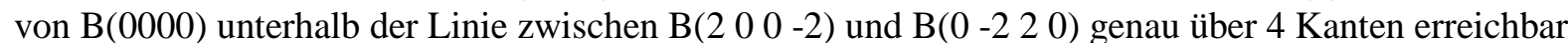
sind, sind türkis umrandet. Analoge Nachbarschaftsverhältnisse wie unterhalb dieser Linie finden sich

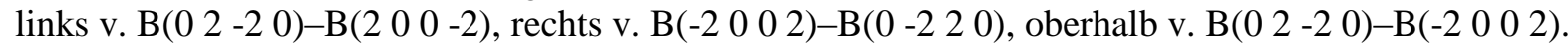


Analoge Nachbarschaftsverhältnisse wie zwischen den Knoten

- unterhalb der Linie zwischen B(2 00 -2) und B( $\left.\begin{array}{llll}0 & -2 & 2 & 0\end{array}\right)$

... gelten für die Bereiche

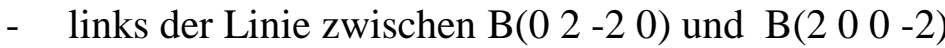

- rechts der Linie zwischen $\mathrm{B}\left(\begin{array}{llll}-2 & 0 & 0 & 2\end{array}\right)$ und $\mathrm{B}\left(\begin{array}{llll}0 & -2 & 2 & 0\end{array}\right)$

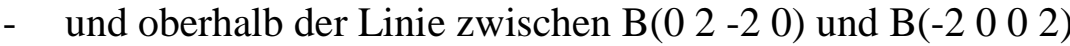

Werden die in diesem Netz miteinander verbundenen Knoten als sich berührende Kugeln betrachtet, können sie auch als Mittelpunktsfigur [Fischer, 1973] bezeichnet werden.

Bei einer solchen Figur wird die Menge all derjenigen Knoten, die von einem Ausgangsknoten den Abstand i besitzen, i-te Kaskade genannt. Sei $\mathrm{k}_{\mathrm{i}}$ die Anzahl der Knoten der i-ten Kaskade, so heißt die Folge $\mathrm{k}_{1}, \mathrm{k}_{2}, \ldots \mathrm{k}_{\mathrm{n}}$ eine Kaskadenfolge $K n$ [Fischer, 1973]. Kaskadenfolgen dienen auch zur Charakterisierung von Netzen.

In der ersten Kaskade des Minimalnetzes 3(4)1 befinden sich vier Knoten, und die Kaskadenfolge $\mathrm{K}_{3}$ (bis zur 3. Schale) lautet: 4, 6, 24.

Abb. 9.7 zeigt einen Vertreter derjenigen Kreisklasse der Länge 8 des Typs 1, in dem der Knoten B viermal vorkommt.

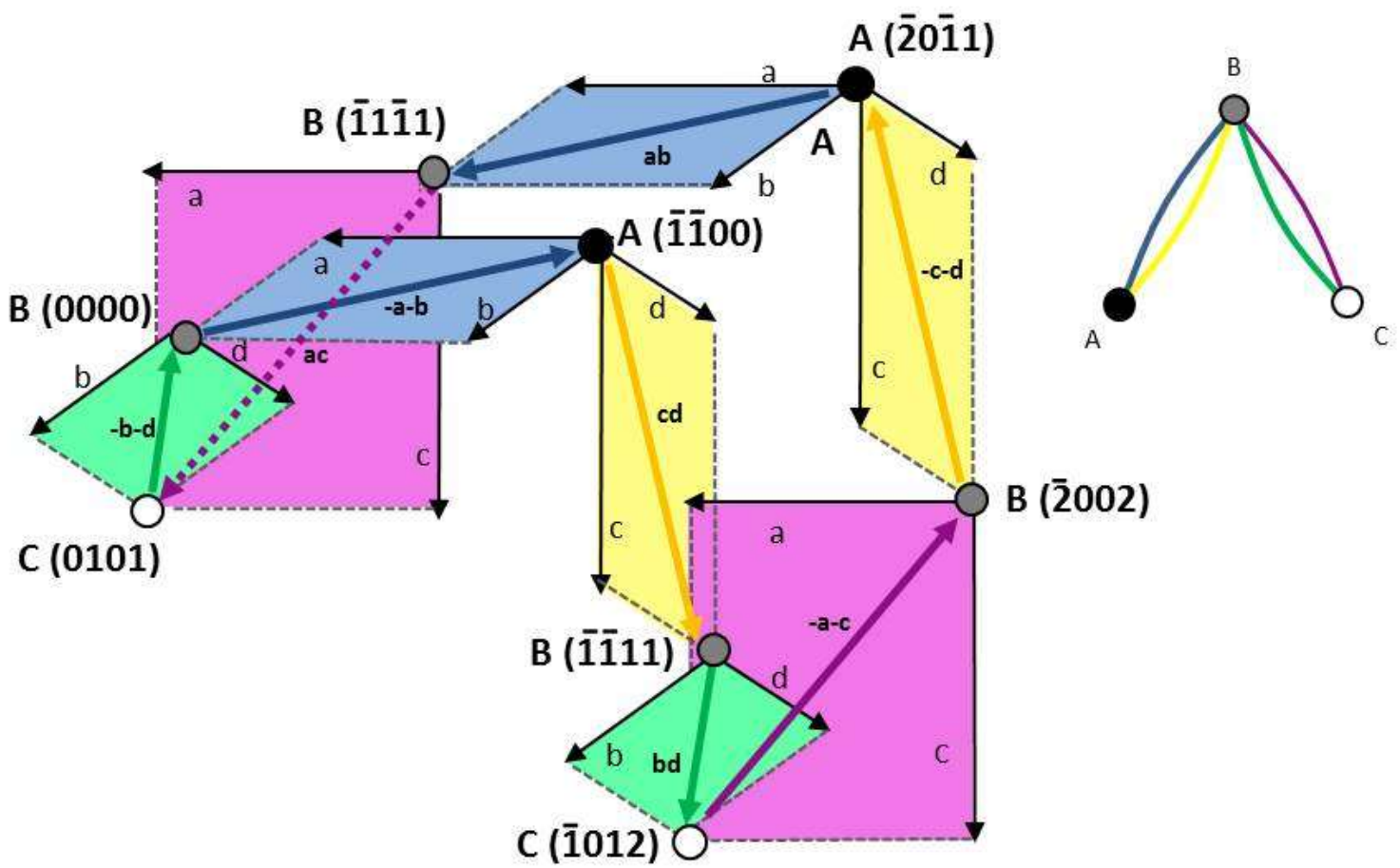

Abb. 9.7 Kreis der Länge 8 im Minimalnetz 3(4)1. Richtung und Längenverhältnisse der Basisvektoren a, b, c, d und der Kanten, sowie die Farbgebung sind an die Abbildung 9.2 angepasst. 
Eine dreidimensionale Abbildung eines 8er-Kreises des vierperiodischen Minimalnetzes 3(4)1 ist im Modell der Abb. 9.8 gezeigt:

- die blauen und gelben Kanten gehören zu einem hyperkubischen Netz mit den Basisvektoren $\mathrm{a}, \mathrm{b}, \mathrm{c}, \mathrm{d}$.

- der rote Faden verbindet die Knoten eines 8er-Kreises (rote Fläche) des Netzes 3(4)1.

- der grüne Faden repräsentiert zwei Vertreter der 2 Kantenklassen, die Knoten C mit Knoten A verbinden.

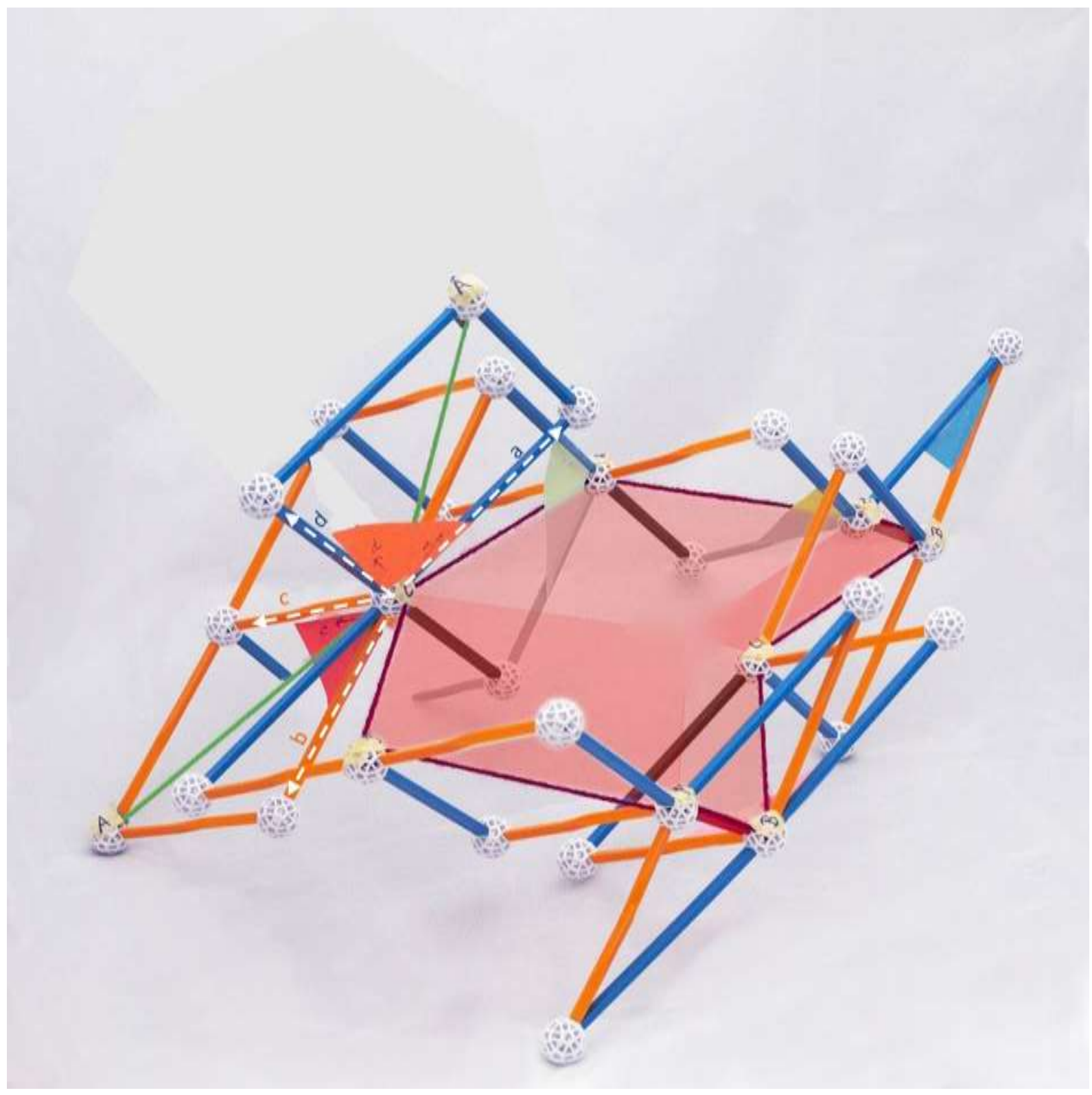

Abb. 9.8 Dreidimensionales Modell - Kreis der Länge 8 im Minimalnetz 3(4)1.

Weiß gestrichelt: Basisvektoren 


\section{Zusammenfassung und Ausblick}

\section{Zusammenfassung}

Die Translationssymmetrie im dreidimensionalen Raum galt bis zur Entdeckung des ersten Quasikristalls als Haupteigenschaft eines kristallinen Zustands. Die „neuen“ Kristalle besitzen dagegen keine Translationssymmetrie im alten Sinn, haben aber eine weitreichende Ordnung in ihrem Aufbau [Elisbihani, 2002].

Die höherdimensionale Kristallographie - die 4-dimensionalen Raumgruppen sind ermittelt [Brown, Bülow, Neubüser, Wondratscheck, Zassenhaus, 1978] - bekommt aufgrund der Erforschung quasiperiodischer Strukturen nun eine ganz andere praktische Bedeutung.

In der vorliegenden Arbeit werden Strukturen mit vier ,örtlichen“ Dimensionen nun unter graphentheoretischen Gesichtspunkten genauer unter die Lupe genommen. Gegenstand der Untersuchungen ist die gesamte Klasse der 4-periodischen Minimalnetze.

Es wurde gezeigt, auf welche Weise sich die Strukturen unterscheiden, und wie Netze durch definierte Umwandlungsprozesse auseinander hervorgehen.

Gemeinsamkeiten und Unterschiede dieser Netze lassen sich durch einen Vergleich ihrer Substrukturen gut erkennen. Alle Bäume, Ketten, Kreisklassen und Minimalnetze geringerer Periodizität sind für die vierperiodischen Minimalnetze aufgeführt:

- Lassen sich für zwei Netze gleiche Bäume finden oder Ketten gleicher Periodenlänge, so sind diese Bäume oder Ketten auf unterschiedliche Weise miteinander verknüpft.

- Die Betrachtung der Kreisklassen kann dazu beitragen, über zweiperiodische Substrukturen einer geeigneten Einbettung eines Netzes näher zu kommen.

- Hat ein vierperiodisches Minimalnetz als Substruktur ein oder mehrere Minimalnetze geringerer Periodizität, so lassen sich diese Netze in entsprechende Unterräume einbetten.

Durch topologische Umwandlungsprozesse können Netze auseinander hervorgehen. Zwei Prinzipien sind beschrieben:

- Minimalnetze lassen sich aus anderen Minimalnetzen gleicher Periodizität erzeugen, durch schrittweise Erhöhung oder Erniedrigung die Ordnung ihrer Quotientengraphen ( $\mathrm{K}_{2}$-Erzeugung und -Verschmelzung).

- Ein vierperiodisches Minimalnetz lässt sich aus seinen dreiperiodischen Netzen, die denselben Quotientengraphen haben, erzeugen. 
Zwei Minimalnetze mit kantentransitiven Quotientengraphen - die Minimalnetze 6(3)1 und 3(4)1 - wurden genauer betrachtet.

- Im Minimalnetz 6(3)1 sind alle Kreise der Länge 12 topologisch gleichwertig. Da sich quasiperiodische Strukturen durch gewisse Parkettierungsmethoden (Penrose-Muster) beschreiben lassen, wurde versucht, eine Teilstruktur dieses Netzes mit Polygonen des Penrose-Musters zur Deckung zu bringen.

Die 12er-Kreise gehören sechs unterschiedlichen Kreisklassen an. Die Kreise einer dieser Kreisklassen können so eingebettet werden, dass sie eine Schar aus ebenen Sechsecknetzen bilden, bei denen sich in den Mitten aller Kanten jeweils ein Knoten des Knotengrades 2 befindet. Einzelne Kreise dieser Kreisklasse wurden so in ein Penrose-Muster eingebettet, dass sie mit einzelnen Polygonen des Musters zur Deckung kommen, und über Kreise anderer Kreisklassen konsistent miteinander verbunden werden können.

- Im Minimalnetz 3(4)1 sind die Kreise der Länge 8 dreier Kreisklassen topologisch gleichwertig. Die 8er-Kreise der 12 übrigen Kreisklassen lassen sich durch Kombinationen aus diesen bilden.

Durch eine besondere Art einer symmetrischen Indizierung der Kanten wurde für das 4-periodische Minimalnetz 3(4)1 eine sehr symmetrische Einbettung in den 4-dimensionalen Raum gefunden, und die Koordinaten der einzelnen Knoten konnten bestimmt werden. Die Entfernungen von einem im „Ursprung“ liegenden Ausgangsknoten zu den innerhalb eines gewissen Bereichs liegenden Knoten wurden ermittelt. Diese Knoten liegen auf konzentrisch um den Ausgangsknoten angeordneten Schalen (Abb. 10.1, links).

Für das Minimalnetz 3(4)3 wurde ein Modell angefertigt (Abb.10.1, i. d. Mitte).

Die für die 4-periodischen Minimalnetze angefertigten perspektivischen Abbildungen (z. B. das Netz 3(4)1 in Abb. 10.1, rechts), basieren auf der Konstruktionen dieser Netze im 3-dimensionalen Anschauungsraum. Validiert werden konnten diese Konstruktionen im Wesentlichen anhand ihrer Kreisklassenspektren.

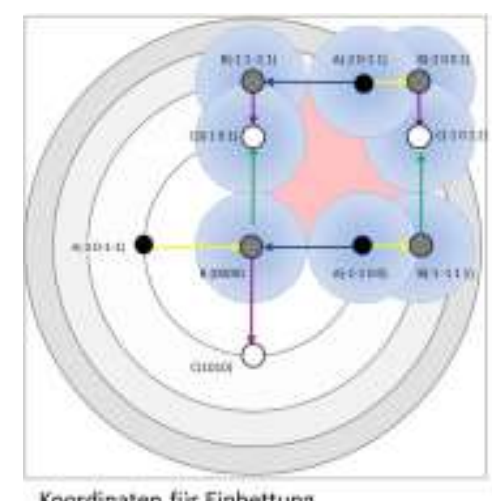

Koordinaten fir Einbettung in den $4 \mathrm{~d}$-Riaum
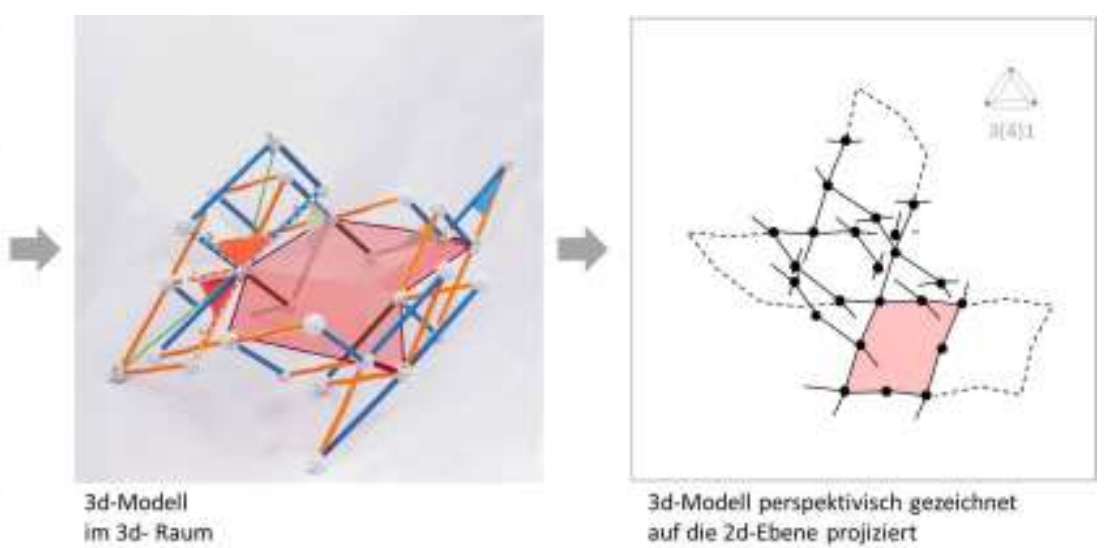

Abb. 10.1 Verschiedene Darstellungen des Minimalnetzes 3(4)3 . Links: Abbildung mit Angabe von 4d-Koordinaten. Mitte: 3d-Modell. Rechts: perspektivische Darstellung auf der Zeichenebene. Es ist jeweils ein Kreis der Länge 8 hellrot markiert. In der linken Abbildung (Ausschnitt aus Abb. 9.6) wurden die Knoten dieses Kreises durch hellblaue Kugeln hinterlegt. 
Die Abbildungen aller 2-, 3- und 4-periodischen Minimalnetze sind im Anhang in Form eines kleinen Nachschlagewerkes zusammengefasst, um eine systematische Suche nach periodischen Netzen, die bestimmte Rahmenbedingungen erfüllen sollen, zu erleichtern. Aufgeführt sind jeweils:

- Quotientengraph

- Abbildung eines Netzausschnitts

- Kreisklassenspektrum

\section{Ausblick}

Die Graphentheorie ist in vielen Wissenschaftsbereichen etabliert - sie können nun auf dieses Nachschlagewerk zurückgreifen. Erwähnen möchte die Elektronik [Weddigen, Jüngst, 1986], die Informatik und die Kommunikationstechnik, mit Schaltungen, Rechner- und Datennetzen, sowie die Architektur und das Bauingenieurwesen mit ihren Tragwerken aus Holz und Metall. Bekannte Beispiele sind der Eiffelturm von Alexandre Gustave Eiffel [Graefe, 1989], die Geodesic Domes von Buckminster Fuller [Marks, 1960] und die Zeltdachkonstruktion (Form und Gestalt: Frei Otto) des Münchner Olympiastadions von Günter Behnisch [Krehl, 1969].

Teilbereiche von Minimalnetzen können eine mögliche Ausgangsbasis zur Erzeugung von unbekannten, komplexen periodischen oder partiell-periodischen Netzstrukturen sein.

Durch das Löschen von bestimmten Bereichen eines 4-periodischen Netzes können auch Substrukturen erzeugt werden, die auf der 3d-Konstruktion dieses 4-periodischen Netzes basieren und somit auf eine vorgegebene Weise in den 3-dimensionalen Raum eingebettet werden.

Eine hypothetische Struktur, bestehend aus Schichten 2-periodischer Strukturen, die durch Ketten durchdrungen werden, ist in Abb. 10.2 dargestellt. Viele Beispiele chemischer Strukturen, die sich durch durchdringende Netze darstellen lassen, sind in [Batten, 2001] aufgelistet. Sich durchdringende 3-periodische Netze wurden von [Delgado-Friedrichs, O’Keeffe, Yaghi, 2002] analysiert.

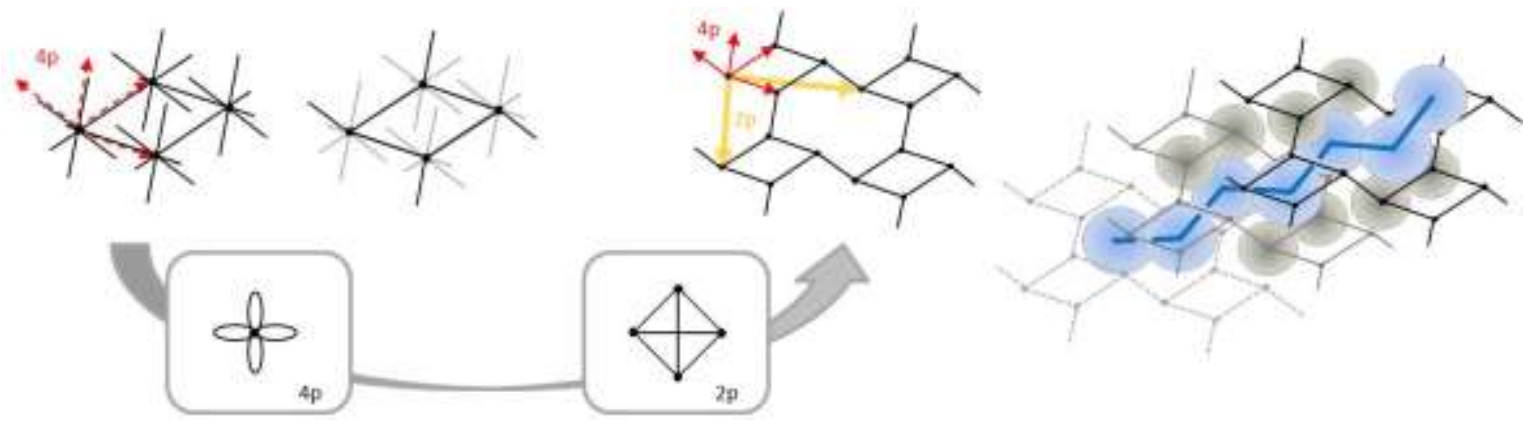

Abb. 10.2 Vierperiodische Strukturen als Ausgangspunkt von 2- oder 3-periodischen Netzen. Aus 2-periodischen Strukturen bestehende Schichten können Substrukturen eines 4p-Minimalnetzes sein. Links oben: 4p-Minimalnetz 1(8)1, Basisvektoren sind rot-gestrichelt, darunter sein QG.

2. Skizze von links (oben): Kanten, die entfernt werden, sind grau gezeichnet; es entsteht ein 2p-Subnetz, rechts darunter sein QG. 3. Skizze von links (oben): größerer Ausschnitt dieses 2p-Subnetzes mit den gelben Pfeilen als Basisvektoren (rote Pfeile: Basisvektoren des ursprünglichen 4p-Minimalnetzes). Es entstehen Bereiche geringerer Dichte, in denen andere Strukturen, z. B. die in der rechten Skizze blau gezeichnete Kette, eingebettet werden können. 
Forschungsgegenstand dieser Arbeit war die Klasse der 4-periodischen Minimalnetze. Aus den durchgeführten Analysen können weitergehende Fragestellungen abgeleitet werden:

1. Das Netz 3(4)1 lässt sich durch eine Mittelpunktsfigur bzw. durch eine Kugelpackung beschreiben und als 4p-Kristallstruktur in den 4-dimensionalen Raum auf geeignete Weise einbetten. Wie sehen die Einbettungen maximaler Symmetrie (Archetypen) [Eon, 1999] und topologische Parameter wie die topologische Dichte [Eon, 2004] für die einzelnen 4-periodischen Minimalnetze aus?

2. Gibt es chemische Umwandlungsprozesse, bei denen sich die Strukturen durch Erhöhung oder Erniedrigung der Ordnung ihrer Quotientengraphen $\left(\mathrm{K}_{2}\right.$-Erzeugung und $\mathrm{K}_{2}$-Verschmelzung) ineinander überführen lassen?

3. Liefern Substrukturen von 4-periodischen Minimalnetzen einen Beitrag zu sich durchdringenden Strukturen, die unter chemischen Gesichtspunkten interessant sind?

4. Penrose-Muster lassen sich aus Substrukturen des 5-periodischen Minimalnetzes 1(10)1 (5d-Gitter) erzeugen (siehe Abb.10.3; vgl. reziproke Basisvektoren in [Steurer, 2004]). Aus welchen vierperiodischen Minimalnetzen lassen sich interessante quasiperiodische Muster erzeugen?

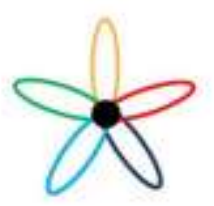

Minimalnetz $1(10) 1$

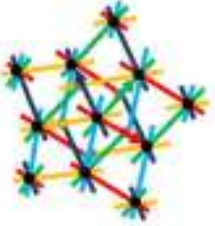

5d-Gitter

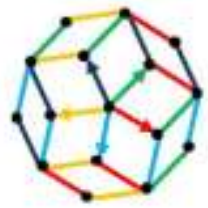

Penrose-Muster (kleiner Ausschnitt)

Abb. 10.3 Penrose-Muster sind Substrukturen des 5p-Minimalnetzes 1(10)1

5. Es gibt Quasikristalle mit quasi-periodischen Teilgittern unterschiedlicher Dimensionalität. Ikosaedrische Quasikristalle haben 3-dimensionale Quasigitter, axiale (oktagonale, dekagonale, dodekagonale) Quasikristalle sind in zwei Dimensionen quasiperiodisch und in der dritten periodisch [Steurer, 2004; Meisterernst, 2006]. Durch entsprechende Prozesse lassen sich periodische Strukturen (Al) auf quasikristalline Oberflächen $\left(\mathrm{Al}_{70} \mathrm{Pd}_{20} \mathrm{Mn}_{10}\right)$ aufbringen [Lüscher, 2004]. Welchen Beitrag können höherperiodische (Minimal-)netze zur Strukturanalyse von Quasikristallen oder zur Beschreibung von Schnittstellen zwischen periodischen und quasiperiodischen Strukturen liefern?

Die vierperiodischen Minimalnetze können Ausgangspunkt zur Erzeugung vieler interessanter 3-dimensionaler Netze, und die Untersuchungen dieser Netze ein Zwischenschritt zur Analyse höherdimensionaler Strukturen (>4) sein. 


\section{Anhang: Die 3 zwei- , die 15 drei- und die 111 vierperiodischen Minimalnetze}

Netzkonstruktionen der zwei-, und dreiperiodischen Minimalnetze sind ab Seite 80, die der vierperiodischen ab Seite 83 dargestellt. Auf einer Seite sind bis zu sechs Netze gezeigt, die Positionen auf einer Seite werden mit a,b, ... bezeichnet und gemäß Abb. A1 angeordnet.

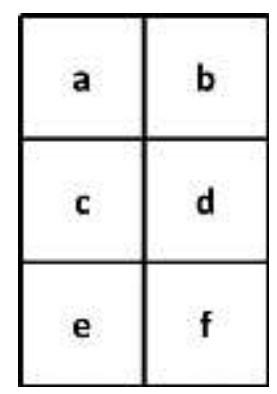

\section{Abb. A1 Positionen auf einer Seite}

Jedes Minimalnetz wird zusammen mit seinem Quotientengraphen und Kreisklassen bis zu einer bestimmten Länge gezeigt (Abb. A2).

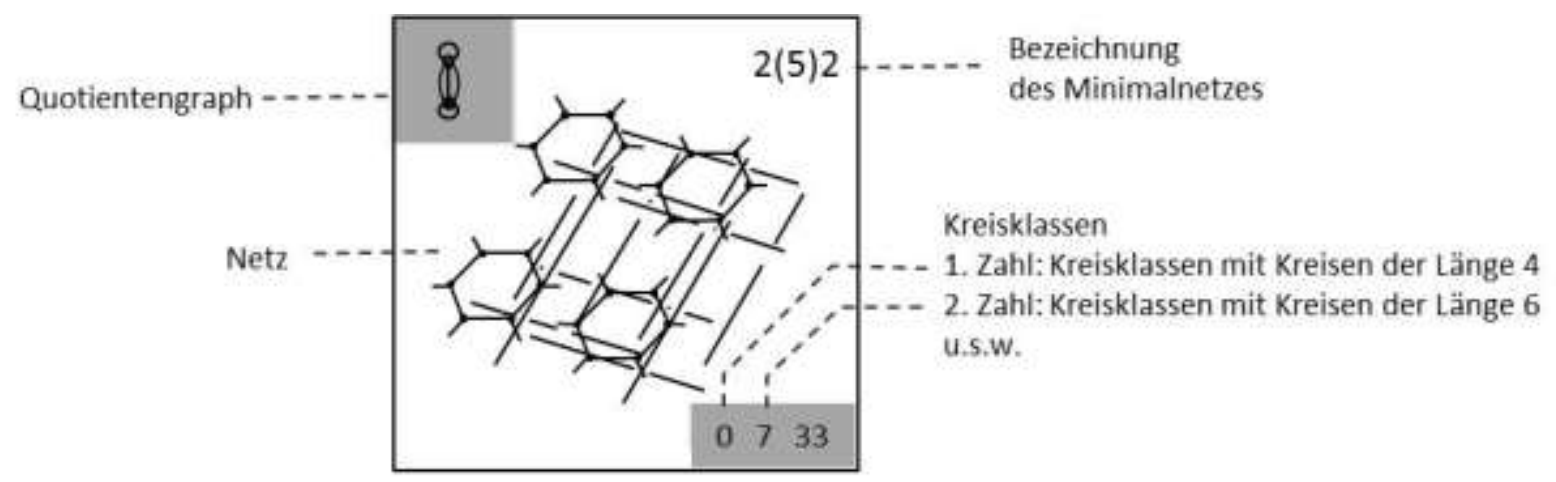

\section{Abb. A2 Minimalnetz mit Quotientengraph und Kreisklassen}

Die Tabellen A1 (für zwei- und dreiperiodische Minimalnetze) und A2 (für vierperiodische Minimalnetze, siehe Seite 83) geben an, auf welcher Seite und Position die einzelnen Minimalnetze zu finden sind.

Periodizität Ordnung Minimalnetze

Anzahl

Seite / Position

\begin{tabular}{|c|c|c|c|c|}
\hline \multirow{2}{*}{2} & 1 & 1(4)1 & 1 & $80 \mathrm{a}$ \\
\hline & 2 & $2(3) 1$ bis $2(3) 2$ & 2 & $80 b-80 c$ \\
\hline \multirow[t]{5}{*}{3} & 1 & $1(6) 1$ & 1 & $80 \mathrm{~d}$ \\
\hline & 2 & $2(4) 1$ bis $2(4) 2$ & 2 & $80 e-80 f$ \\
\hline & & $2(3,5) 1$ bis $2(3,5) 2$ & 2 & $81 a-81 b$ \\
\hline & 3 & $3\left(3^{2}, 4\right) 1$ bis $3\left(3^{2}, 4\right) 5$ & 5 & $81 c-82 a$ \\
\hline & 4 & $4(3) 1$ bis $4(3) 5$ & 5 & $82 b-82 f$ \\
\hline
\end{tabular}

Tabelle A1 Seite und Position der zwei- und dreiperiodischen Minimalnetze 


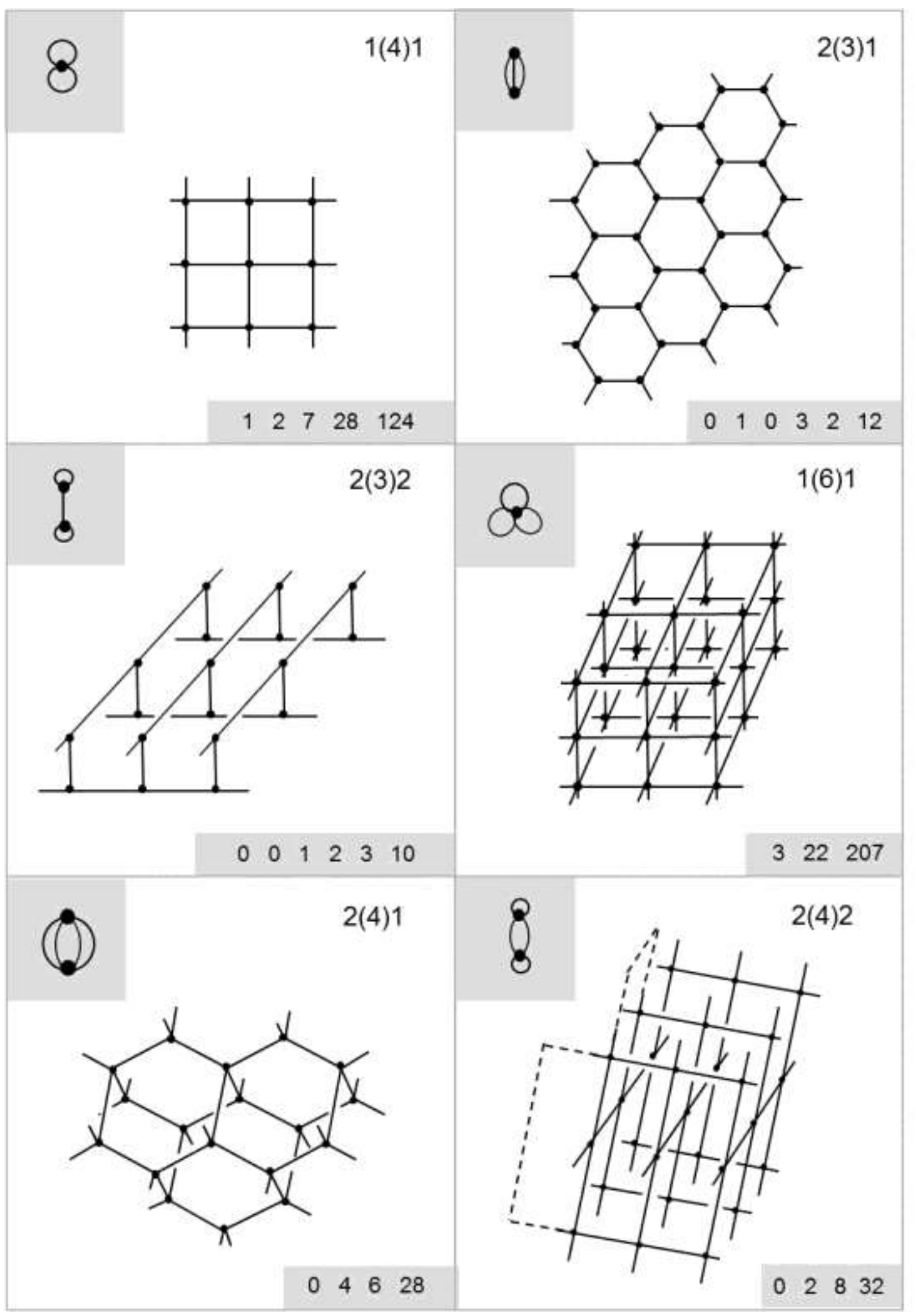




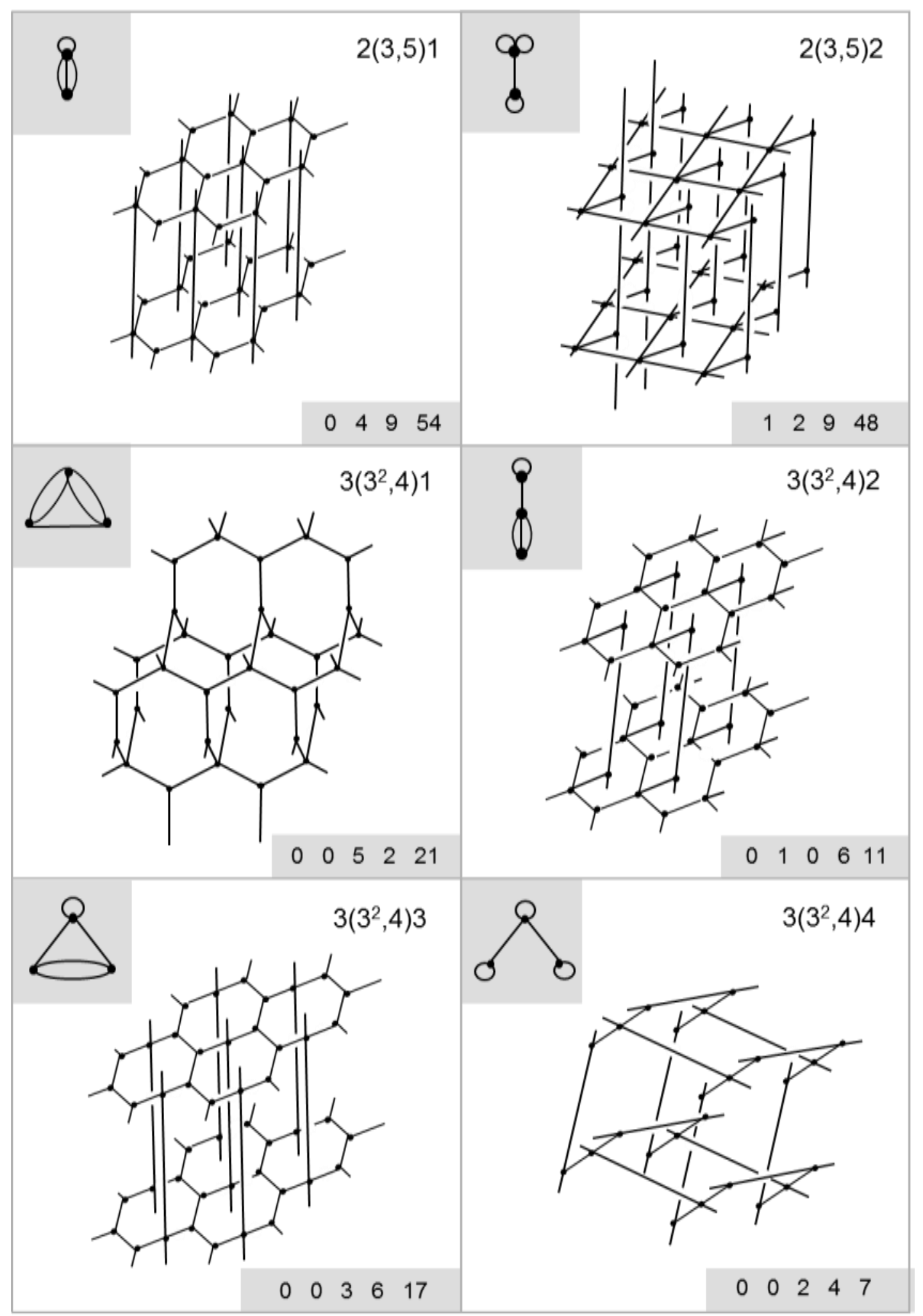




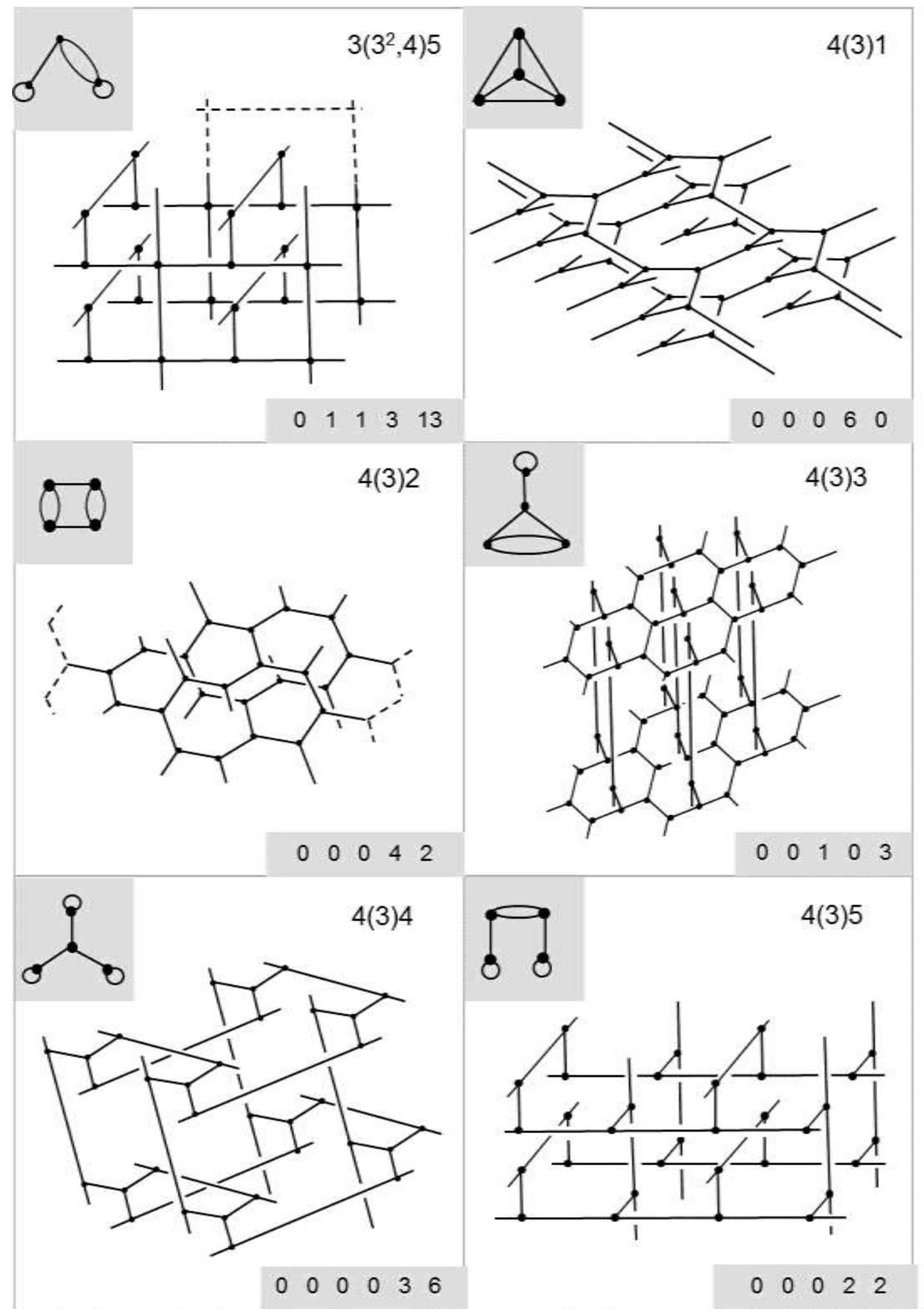


Die Tabelle A2 gibt an, auf welcher Seite und Position die einzelnen vierperiodischen Minimalnetze zu finden sind.

Periodizität Ordnung Minimalnetze

Anzahl

Seite / Position

4

1

3

3

$3(4) 1$ bis $3(4) 2$

$3(3,4,5) 1$ bis $3(3,4,5) 10$

4

$4\left(3^{2}, 4^{2}\right) 1$ bis $4\left(3^{2}, 4^{2}\right) 22$

5

6 $3\left(3^{2}, 6\right) 1$ bis $3\left(3^{2}, 6\right) 6$

$4\left(3^{3}, 5\right) 1$ bis $4\left(3^{3}, 5\right) 14$

1(8) 1

2(5) 1 bis $2(5) 3$

$2(3,7) 1$ bis $2(3,7) 2$

$2(4,6) 1$ bis $2(4,6) 2$

$5\left(3^{4}, 4\right) 1$ bis $5\left(3^{4}, 4\right) 30$

6(3) 1 bis 6(3)17
1

$83 \mathrm{a}$

$83 b-84 b$

$84 \mathrm{c}-84 \mathrm{~d}$

$84 \mathrm{e}-84 \mathrm{f}$

$85 \mathrm{a}-85 \mathrm{~d}$

$86 \mathrm{a}-86 \mathrm{f}$

$87 \mathrm{a}-88 \mathrm{~d}$

$89 a-91 b$

$91 \mathrm{c}-94 \mathrm{f}$

$95 \mathrm{a}-99 \mathrm{f}$

$100 \mathrm{a}-102 \mathrm{e}$

Tabelle A2 Seite und Position der vierperiodischen Minimalnetze

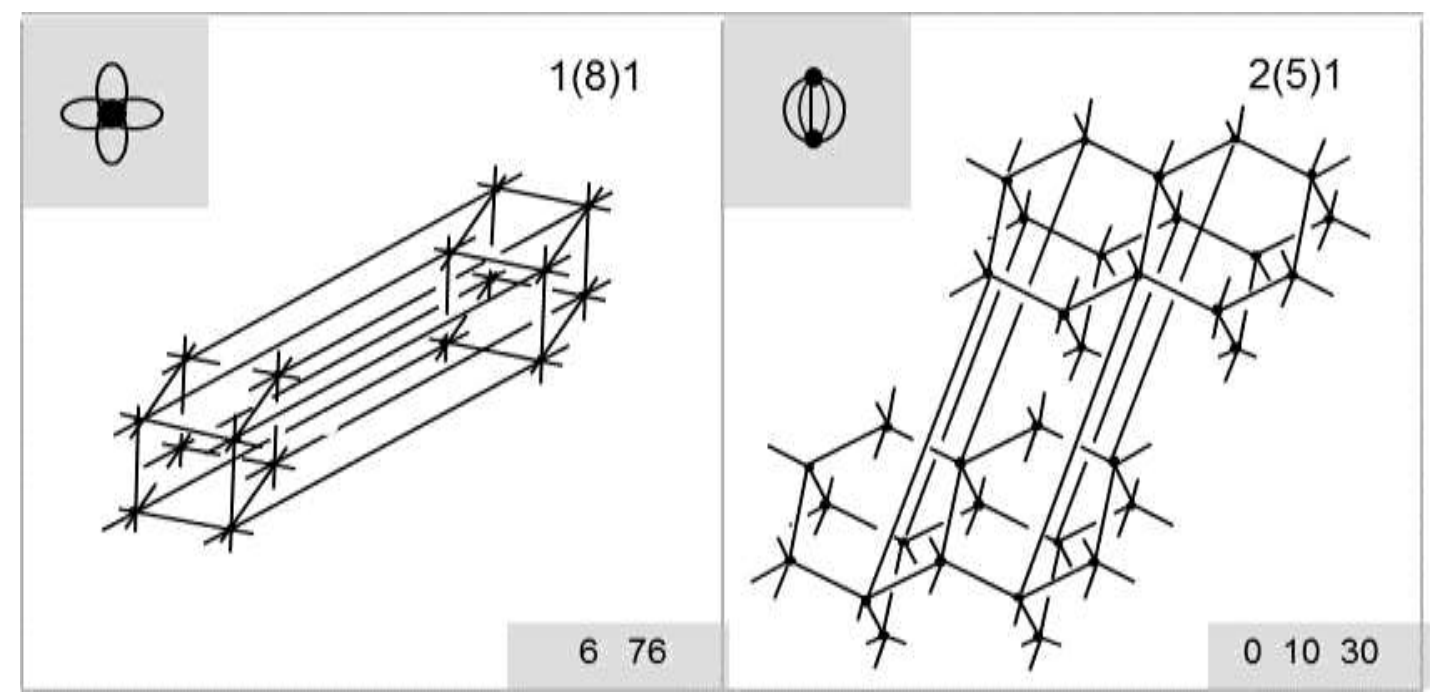




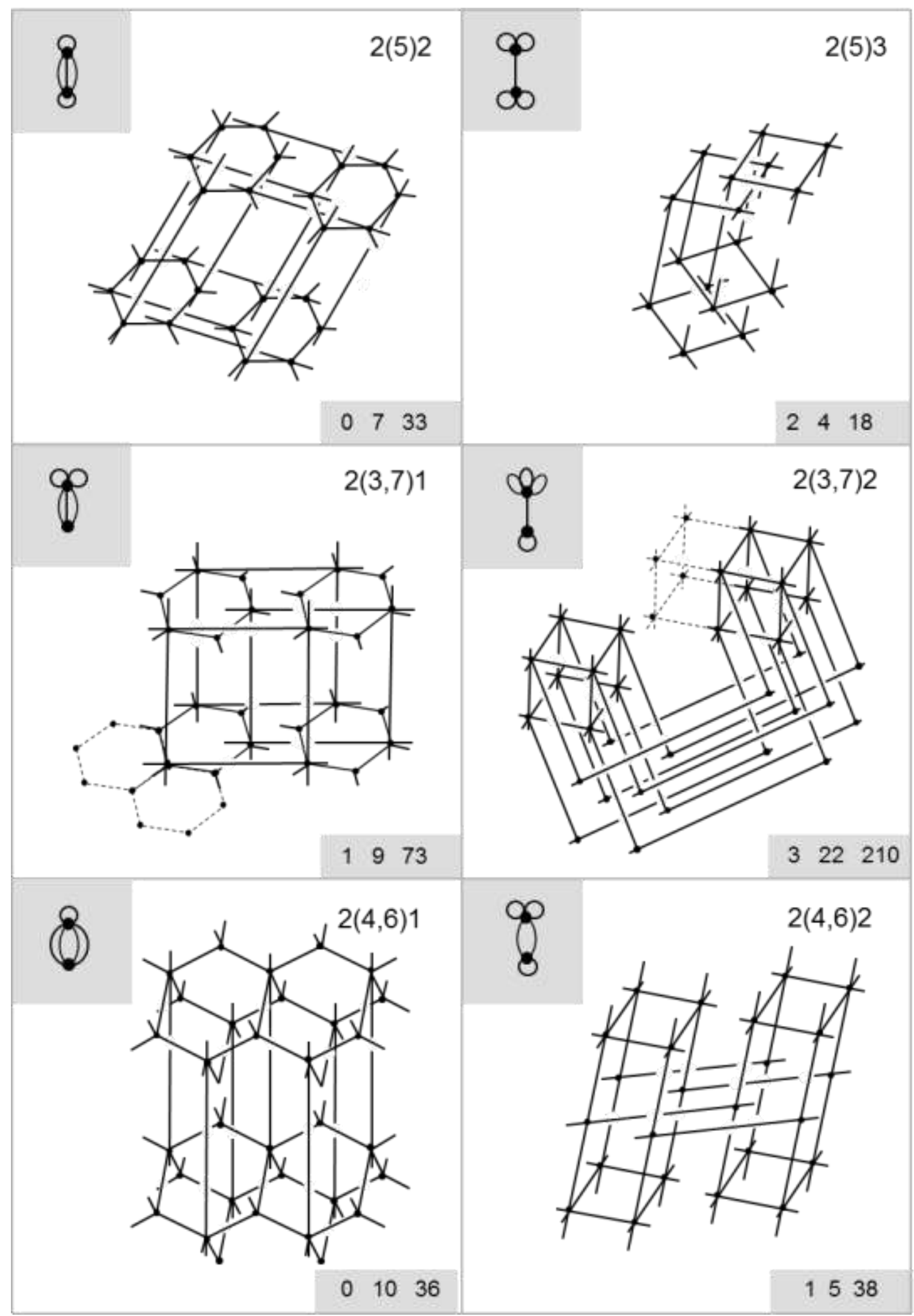




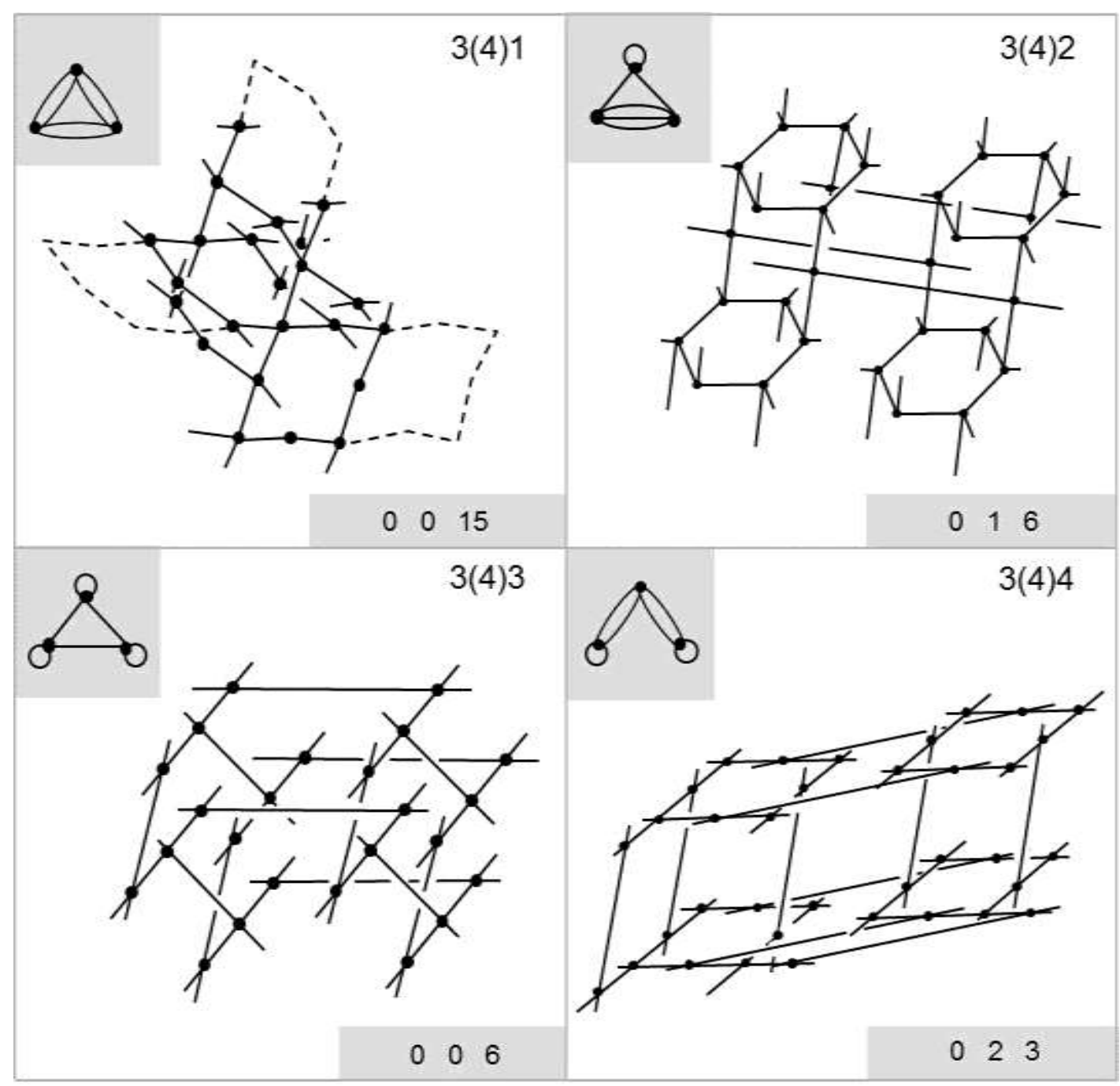




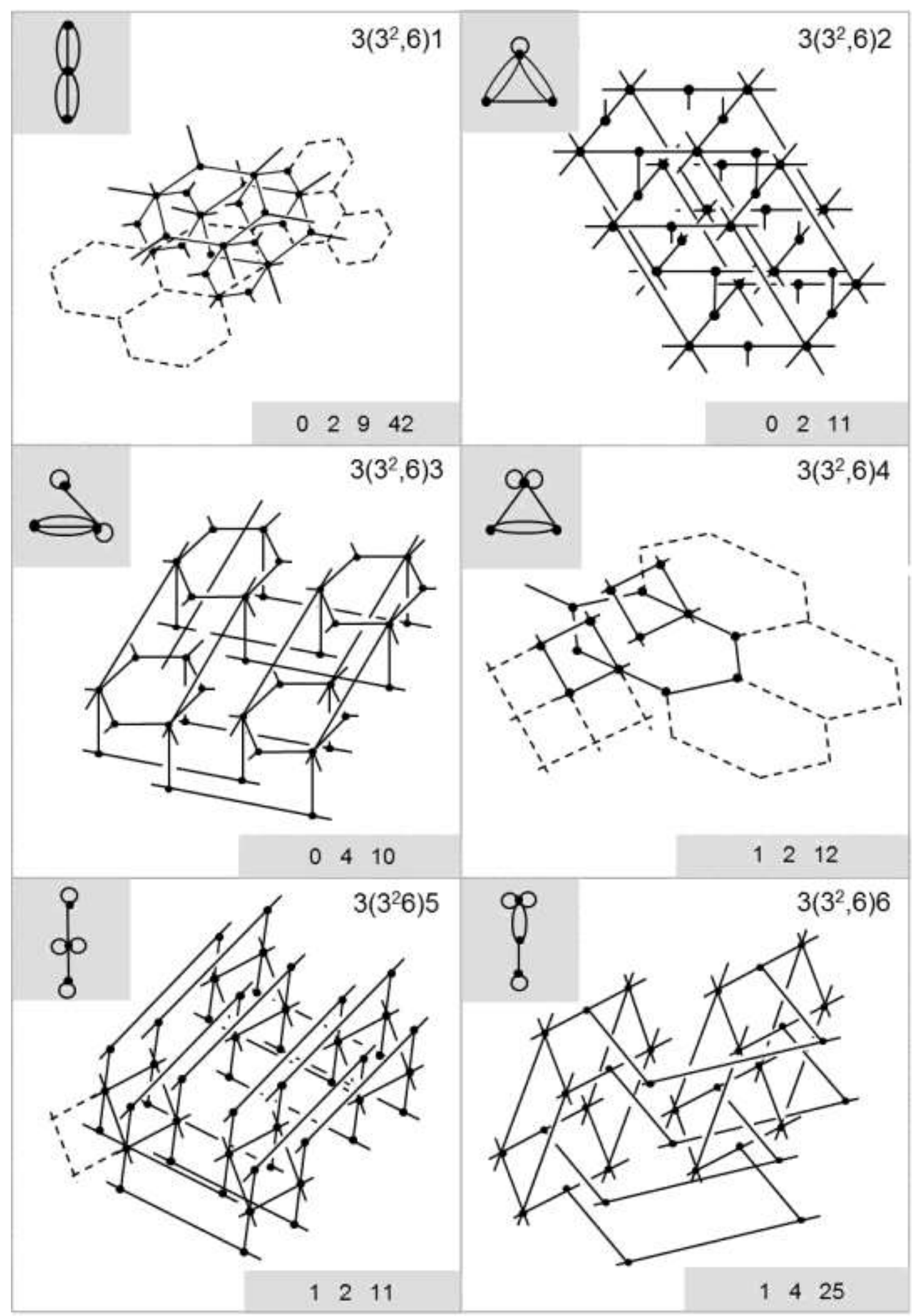




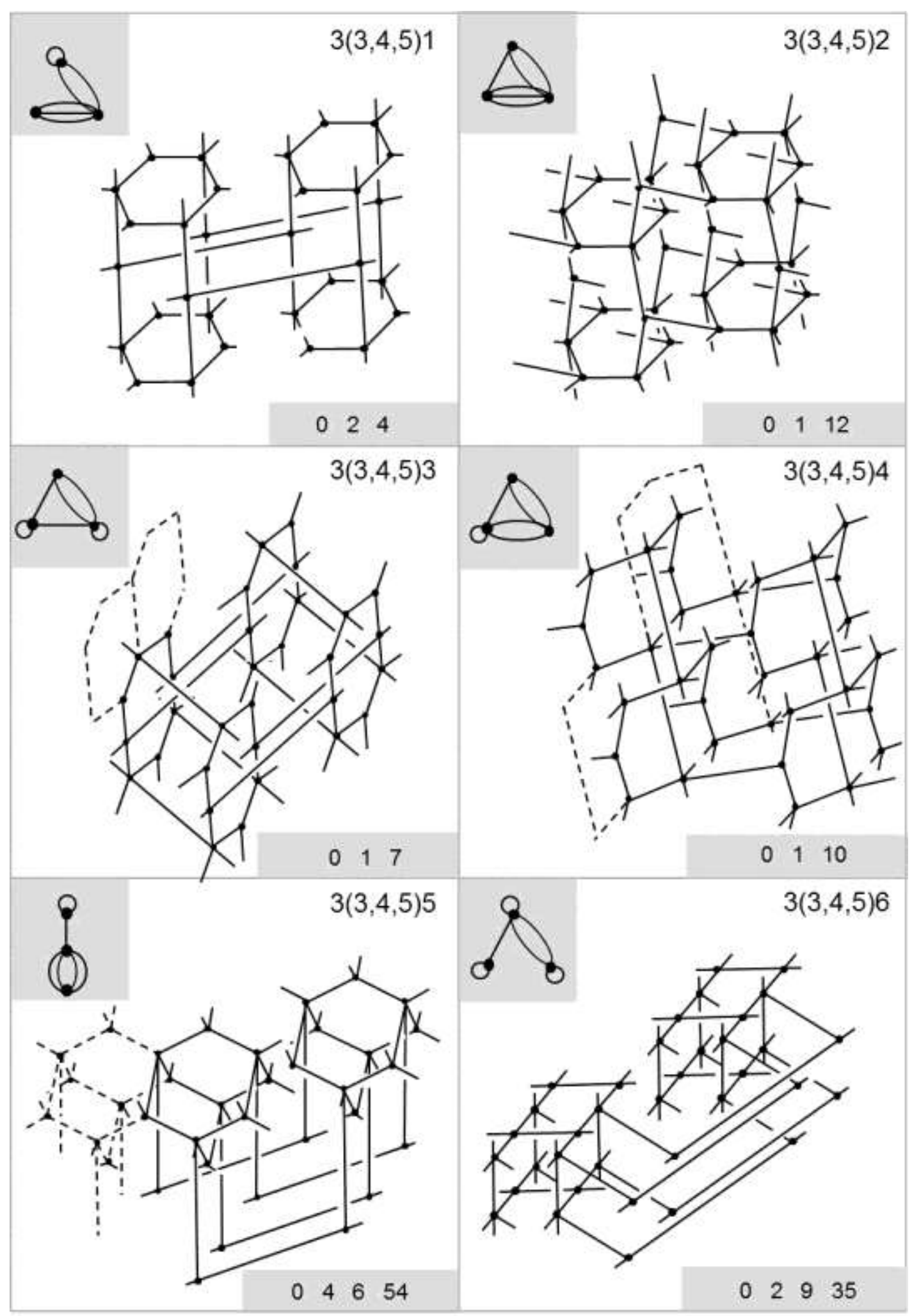




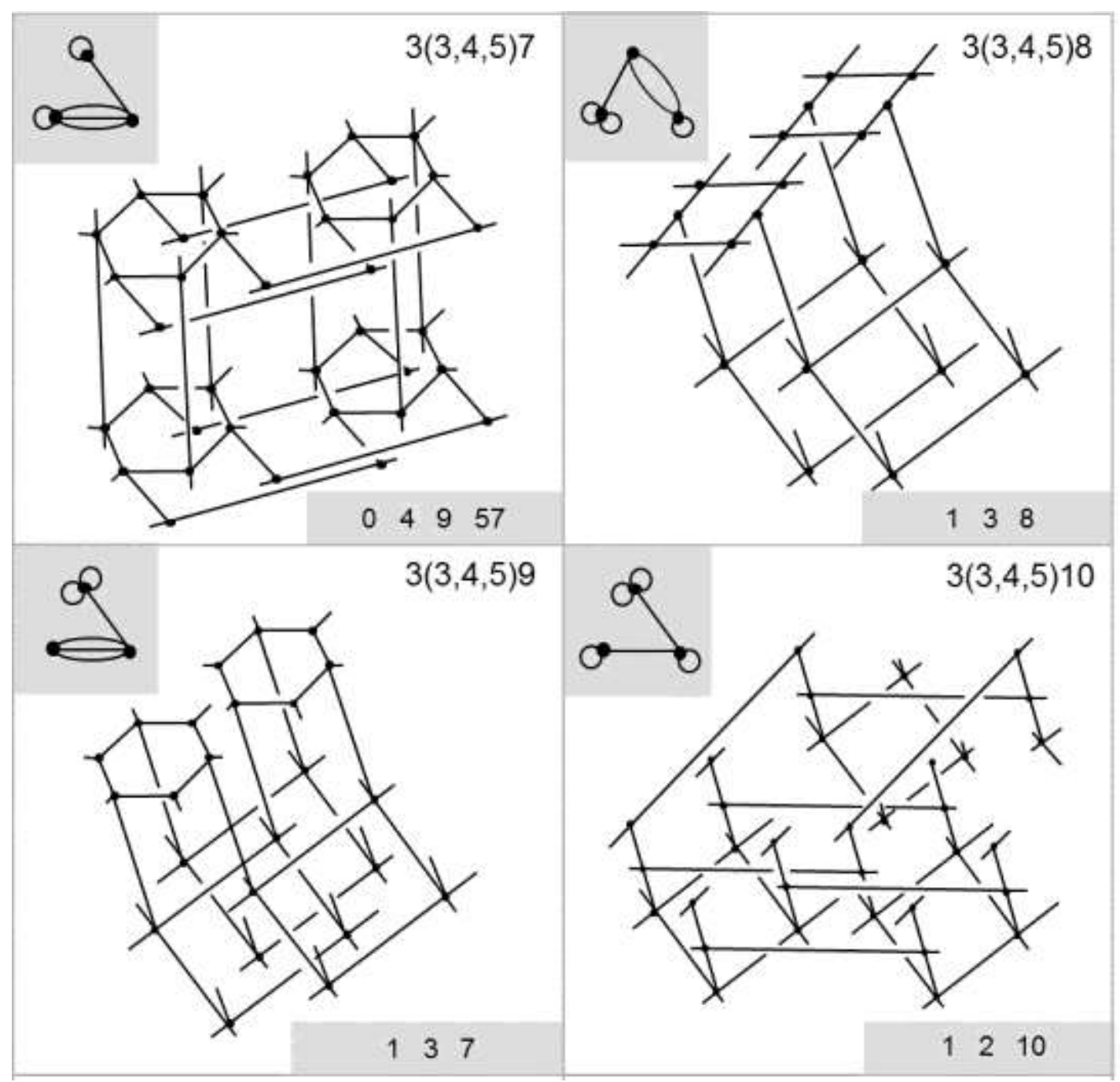




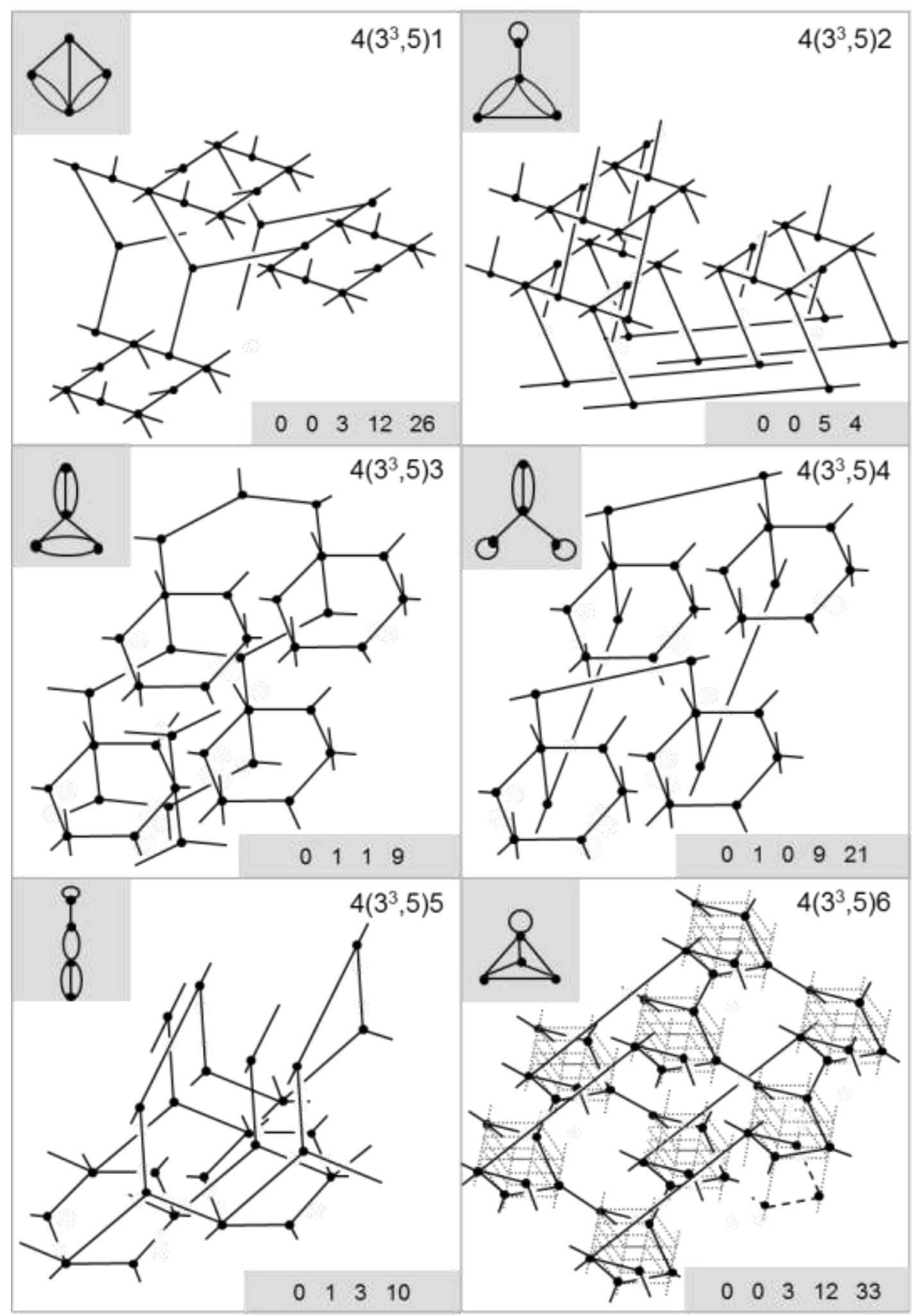




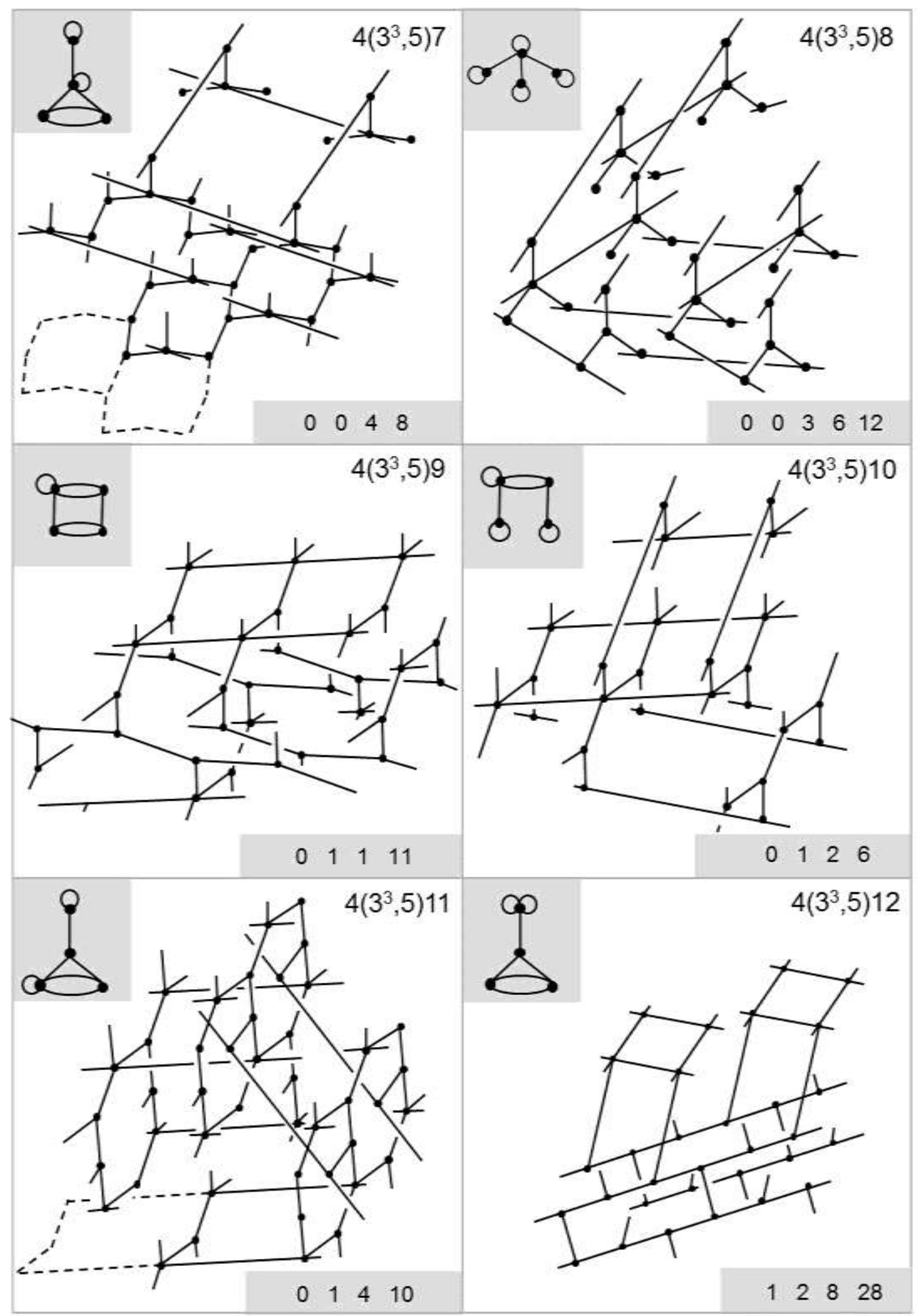




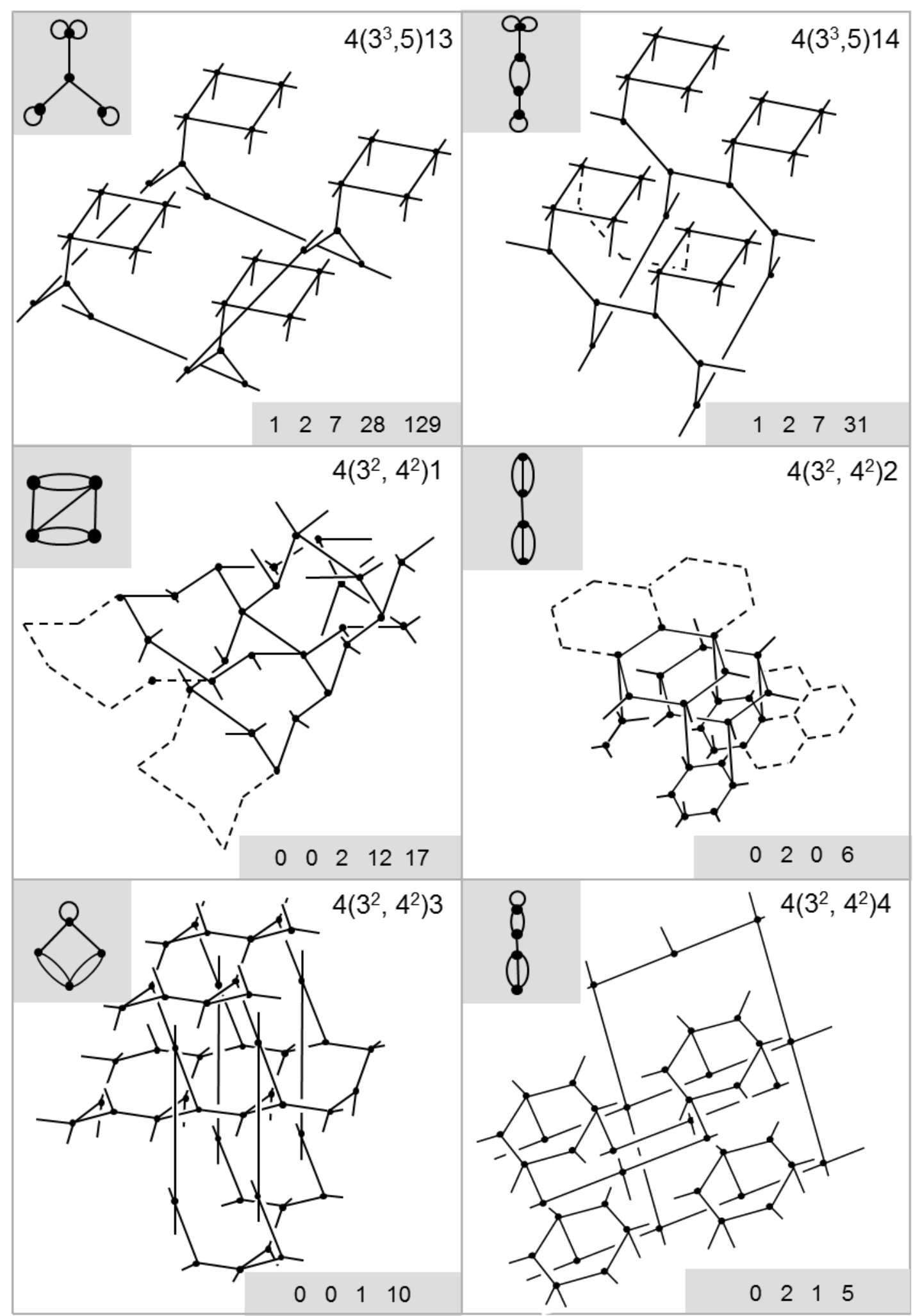




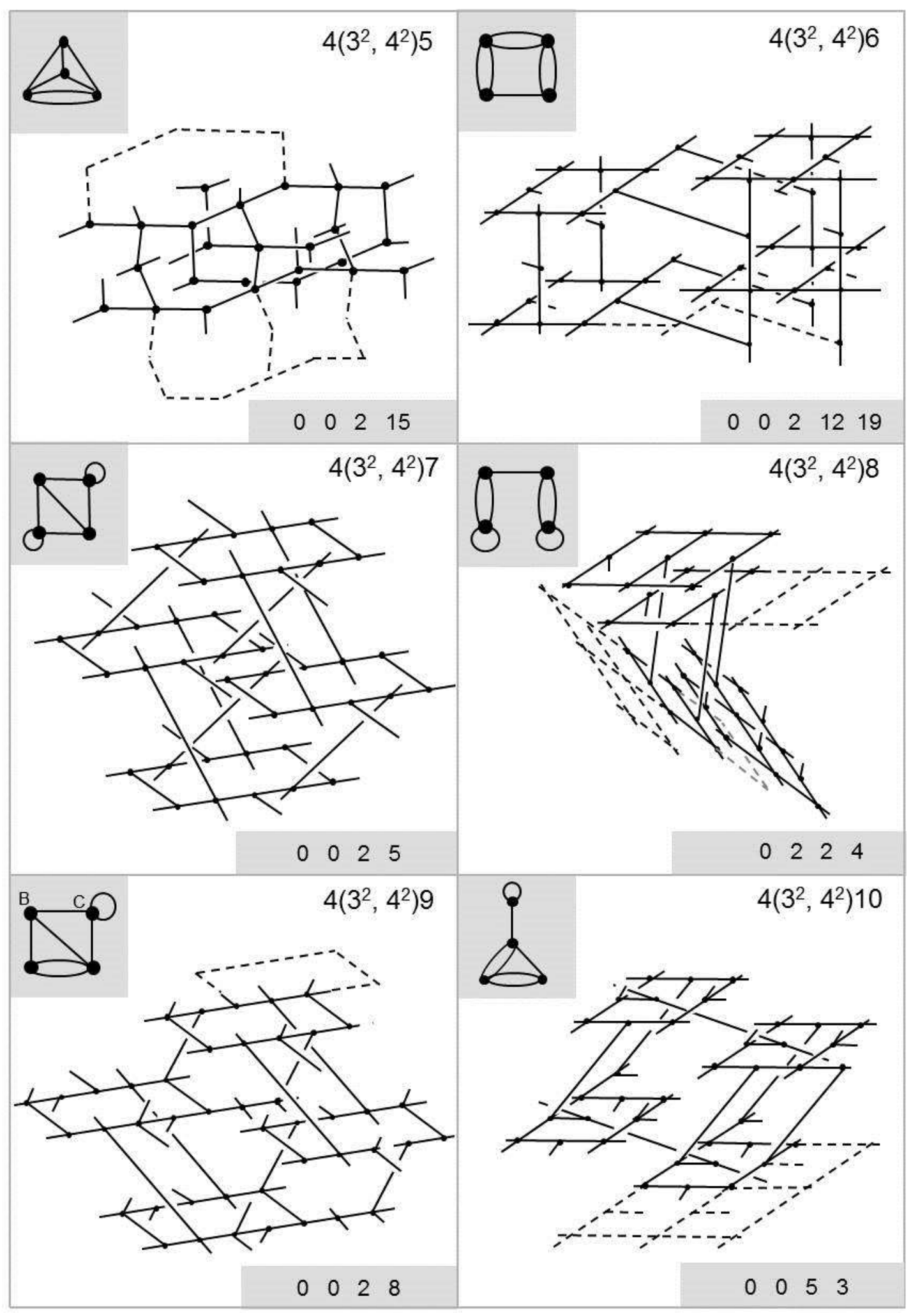




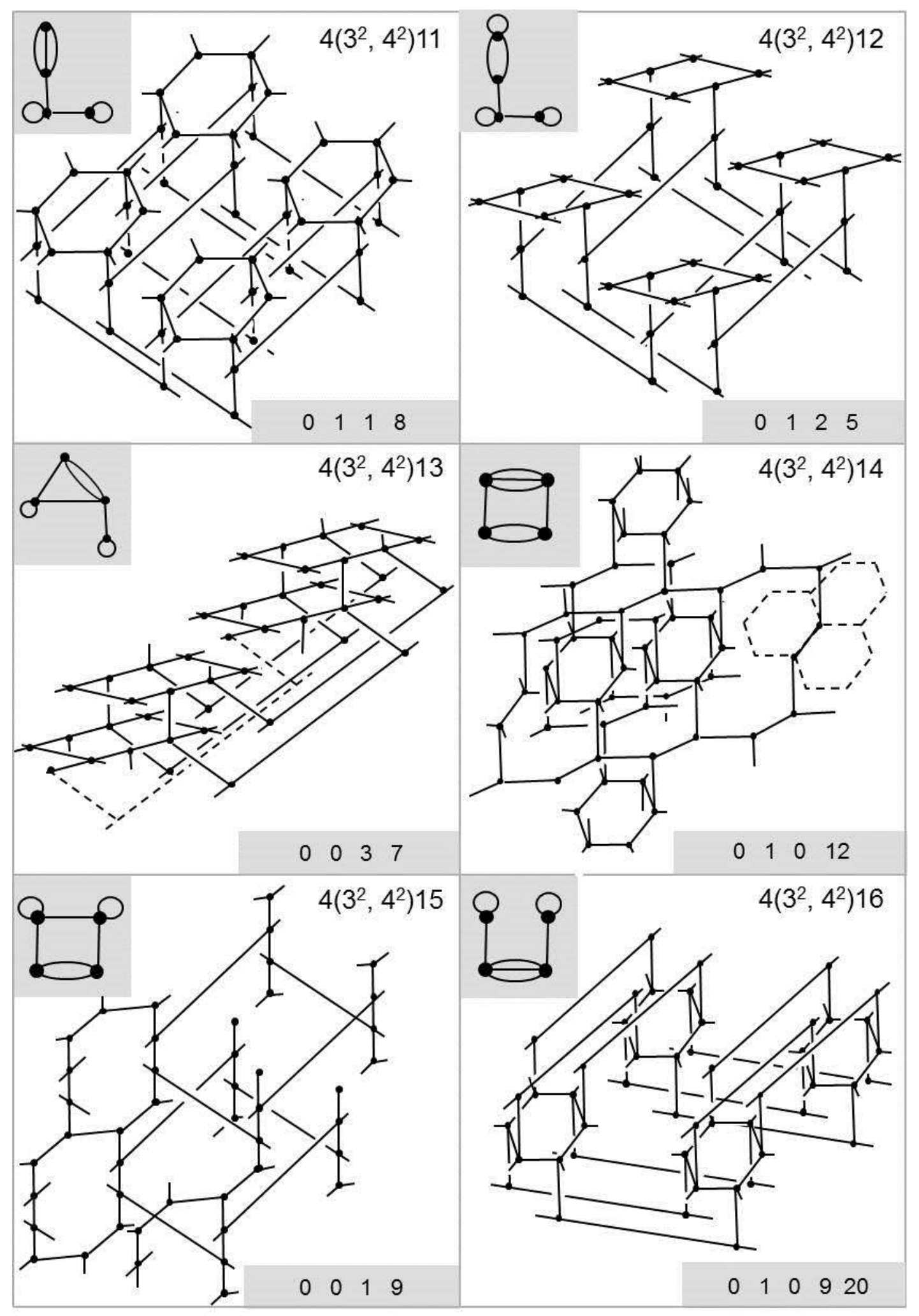




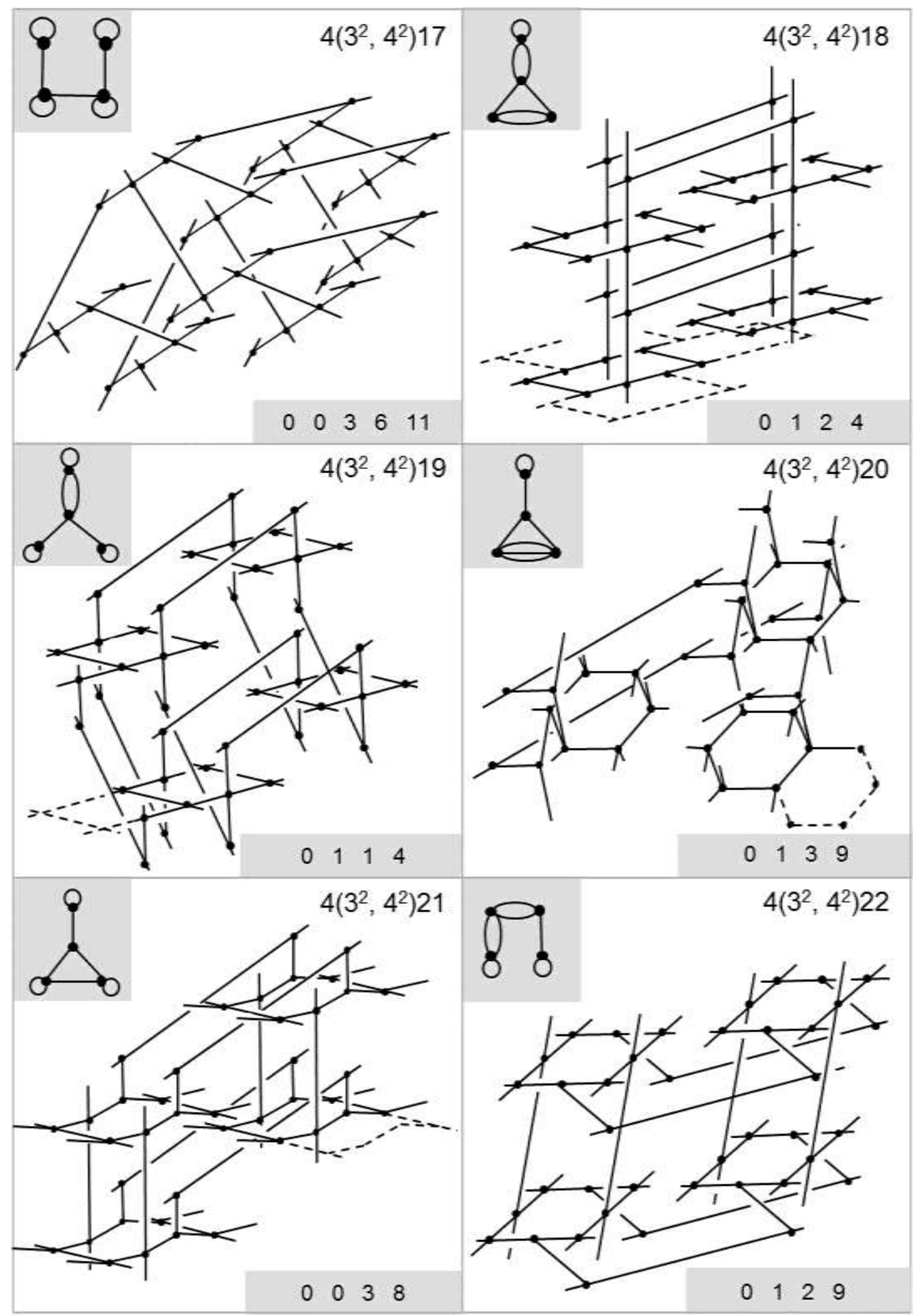




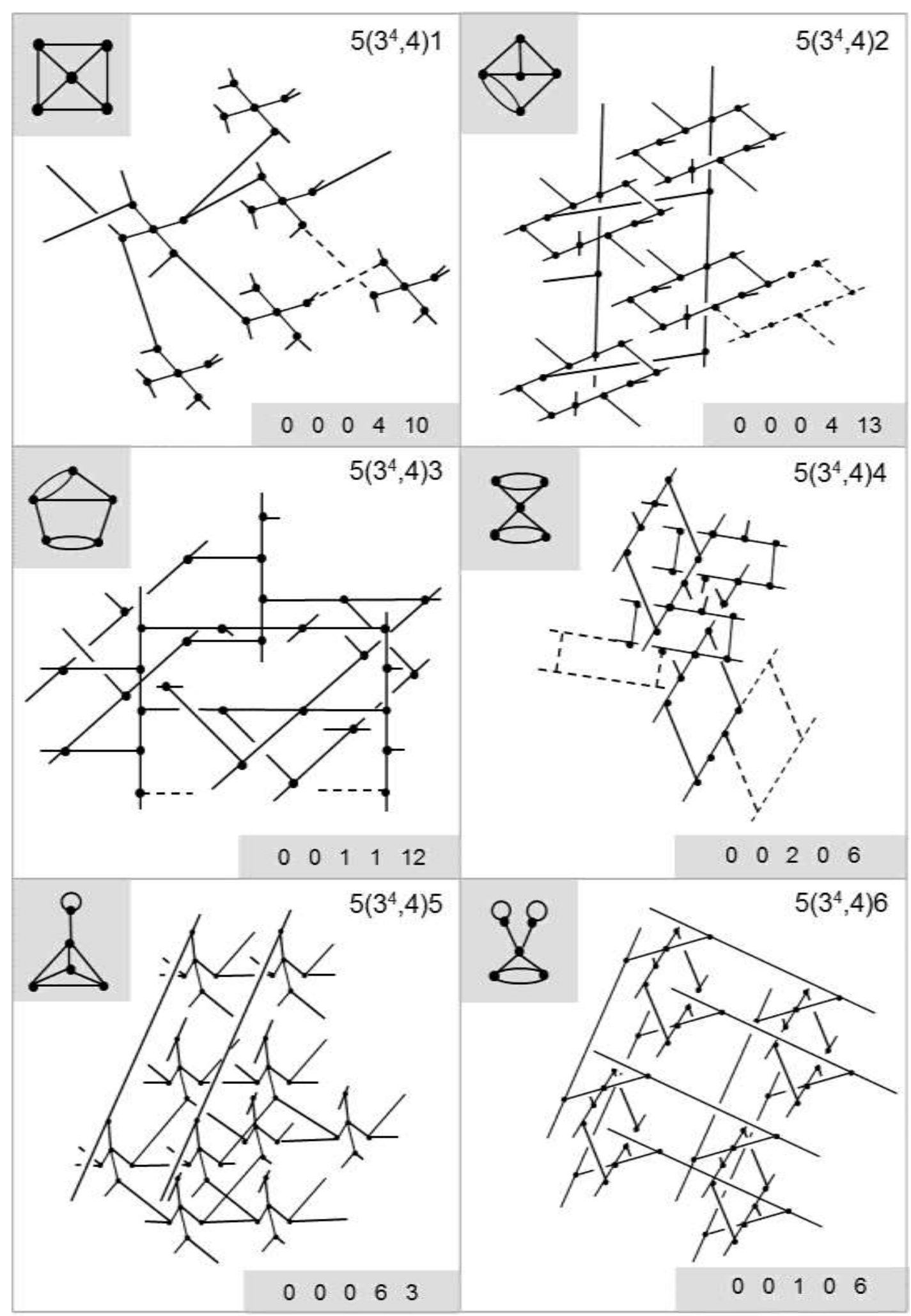




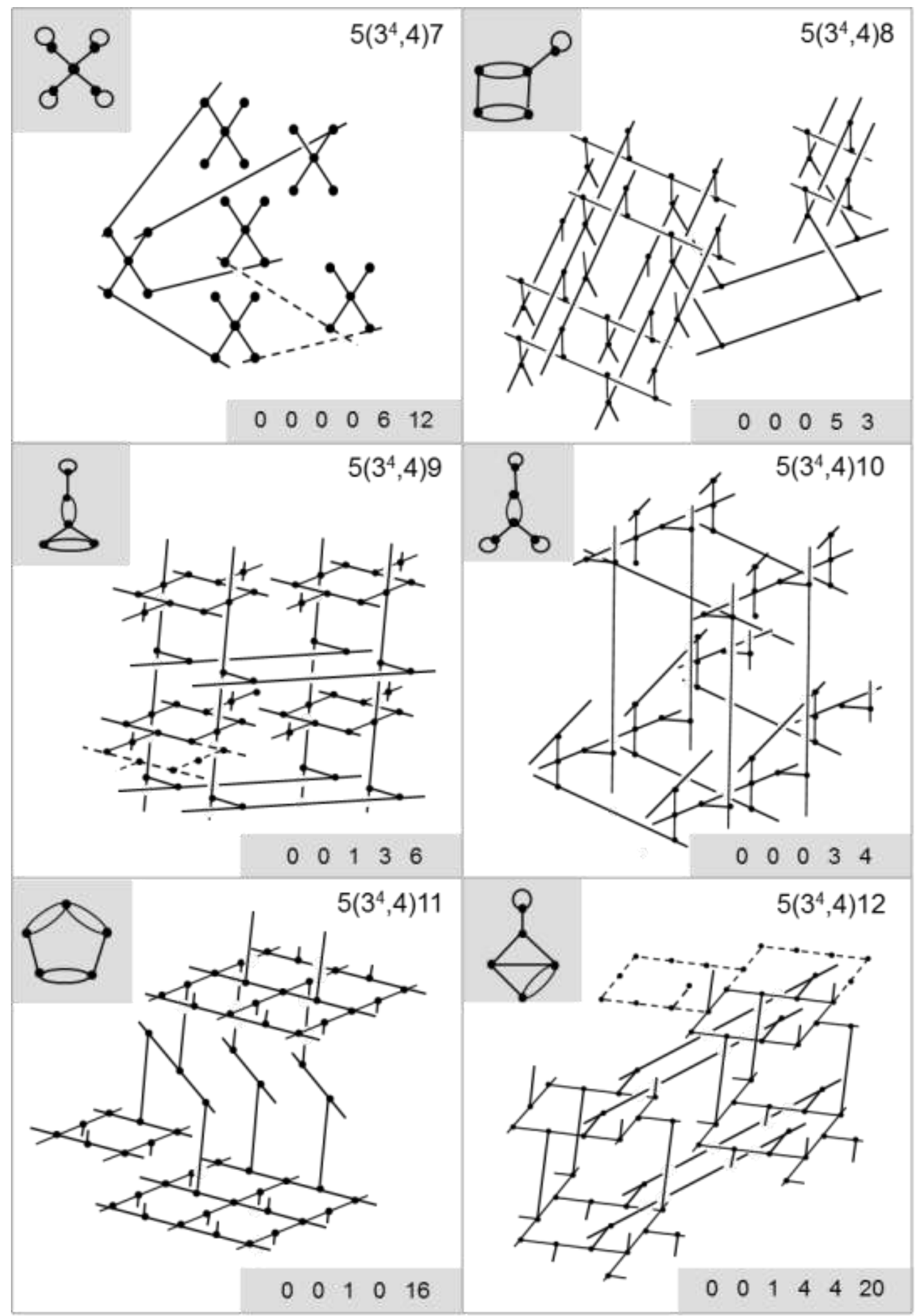




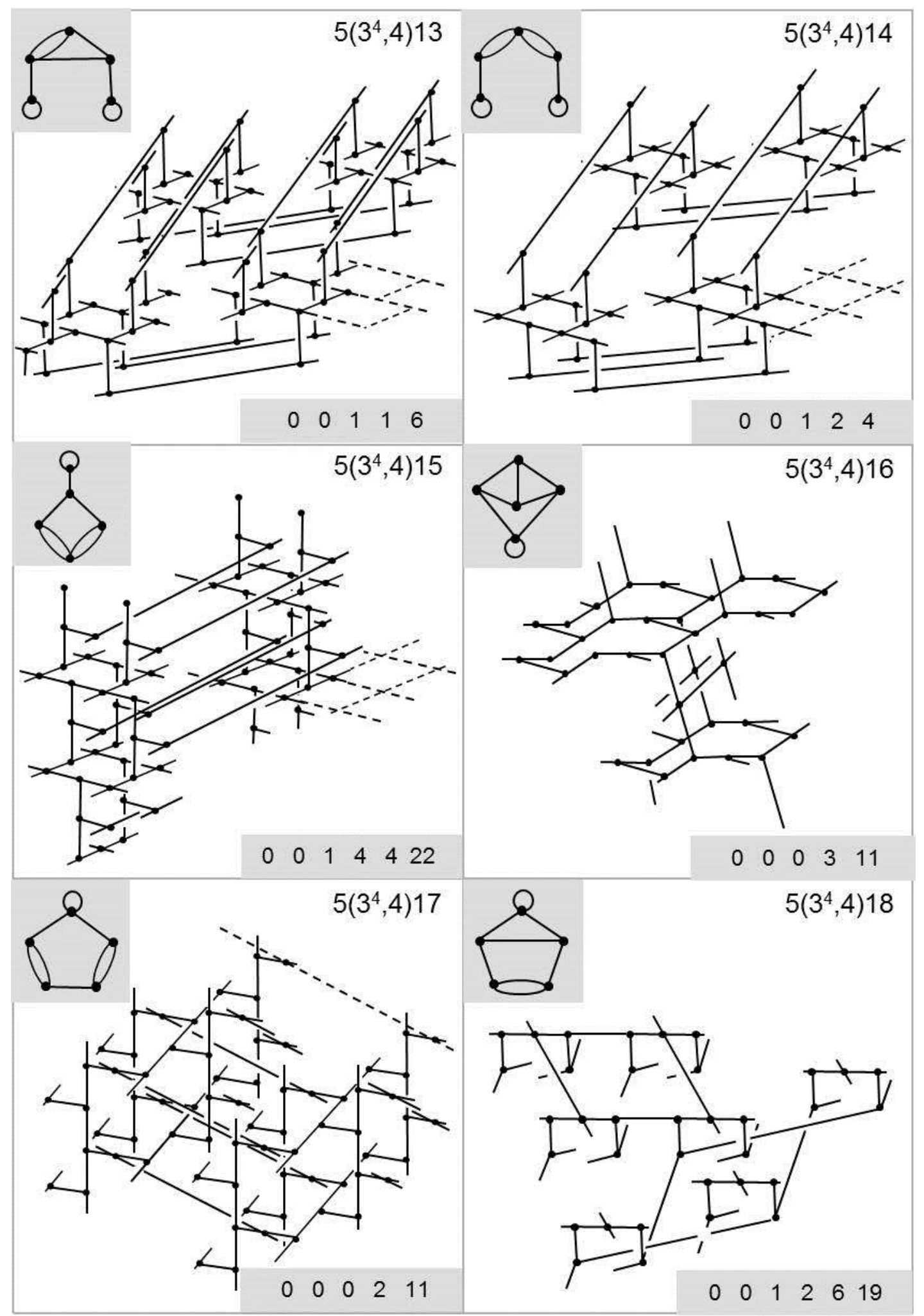




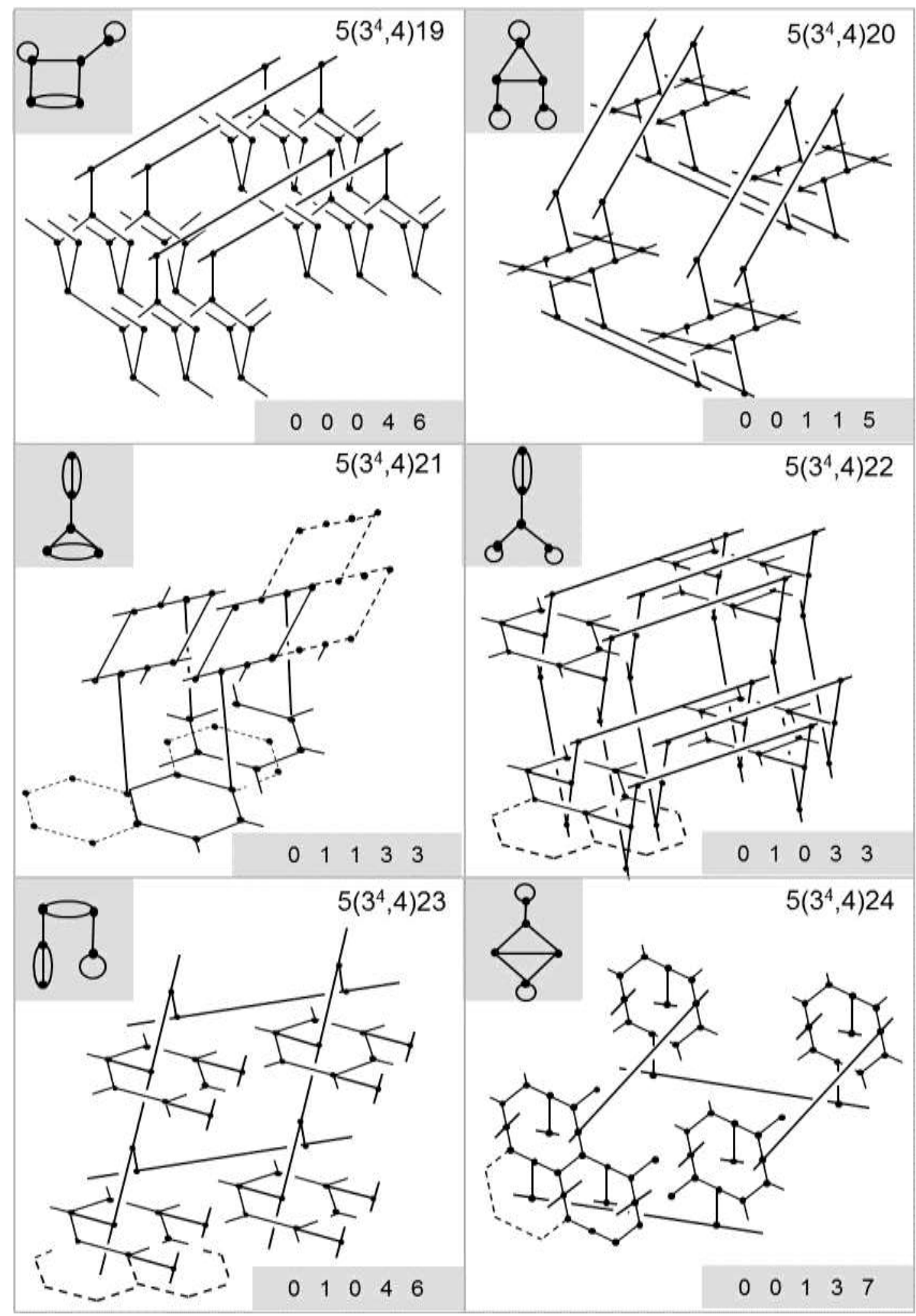




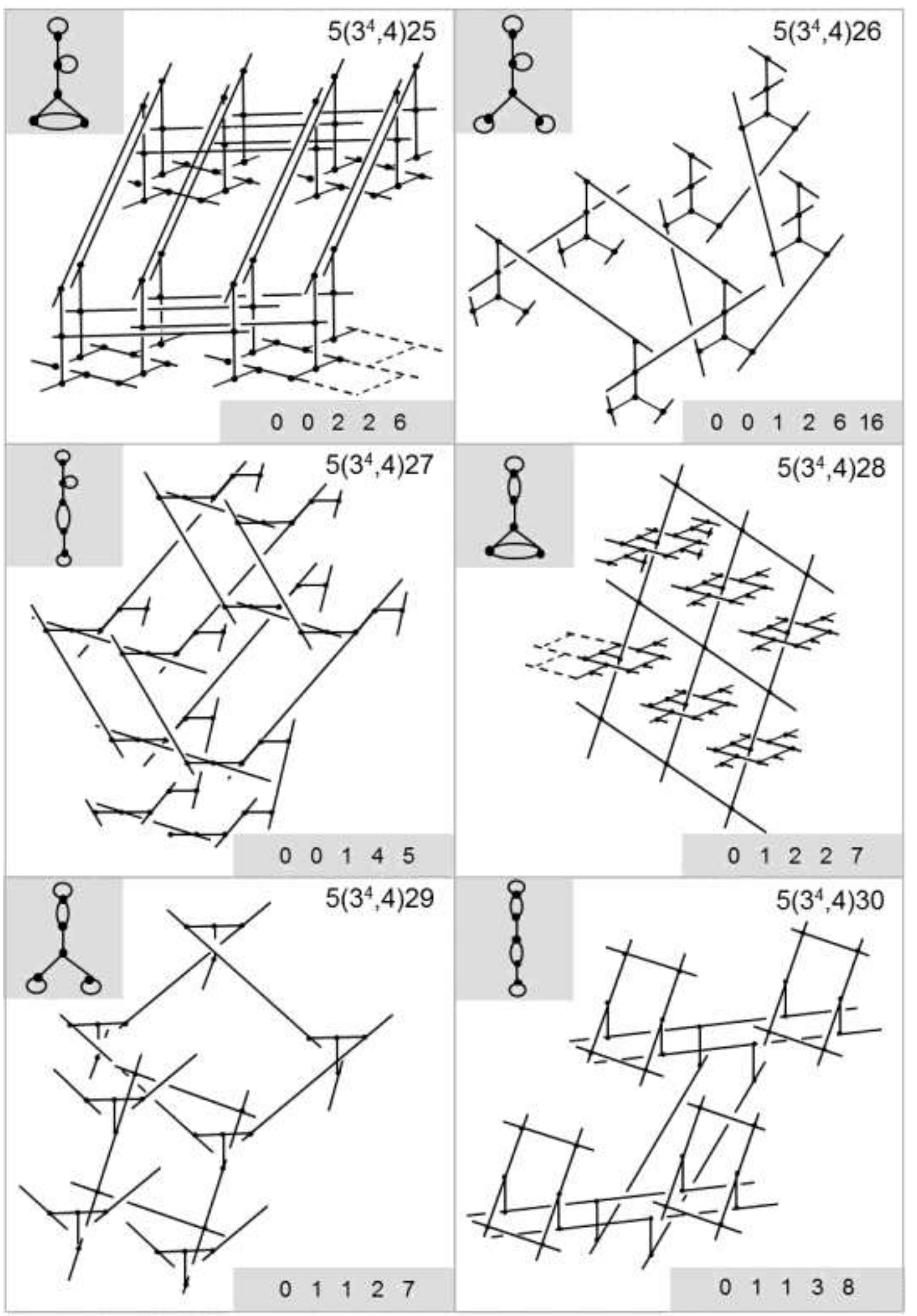




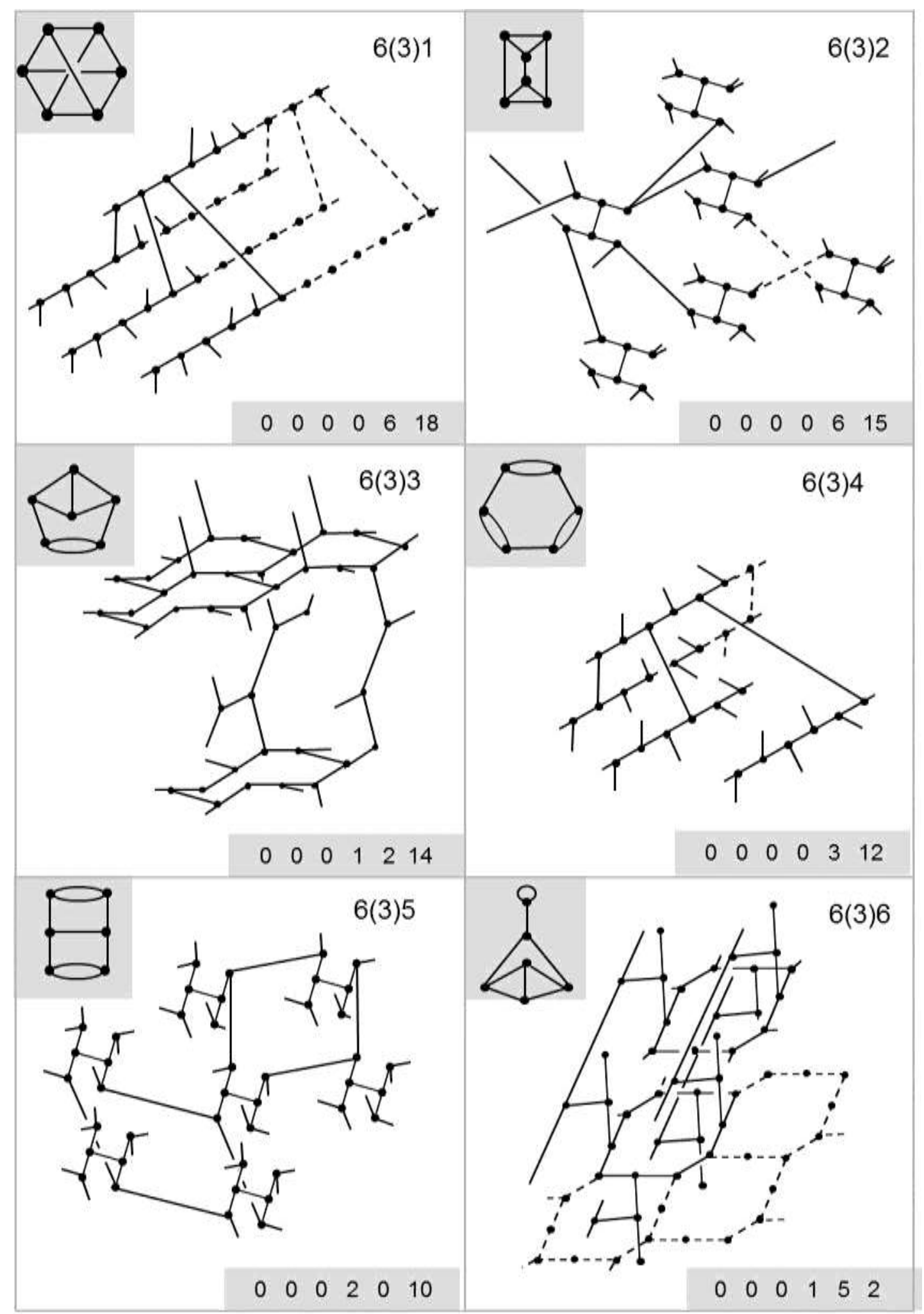




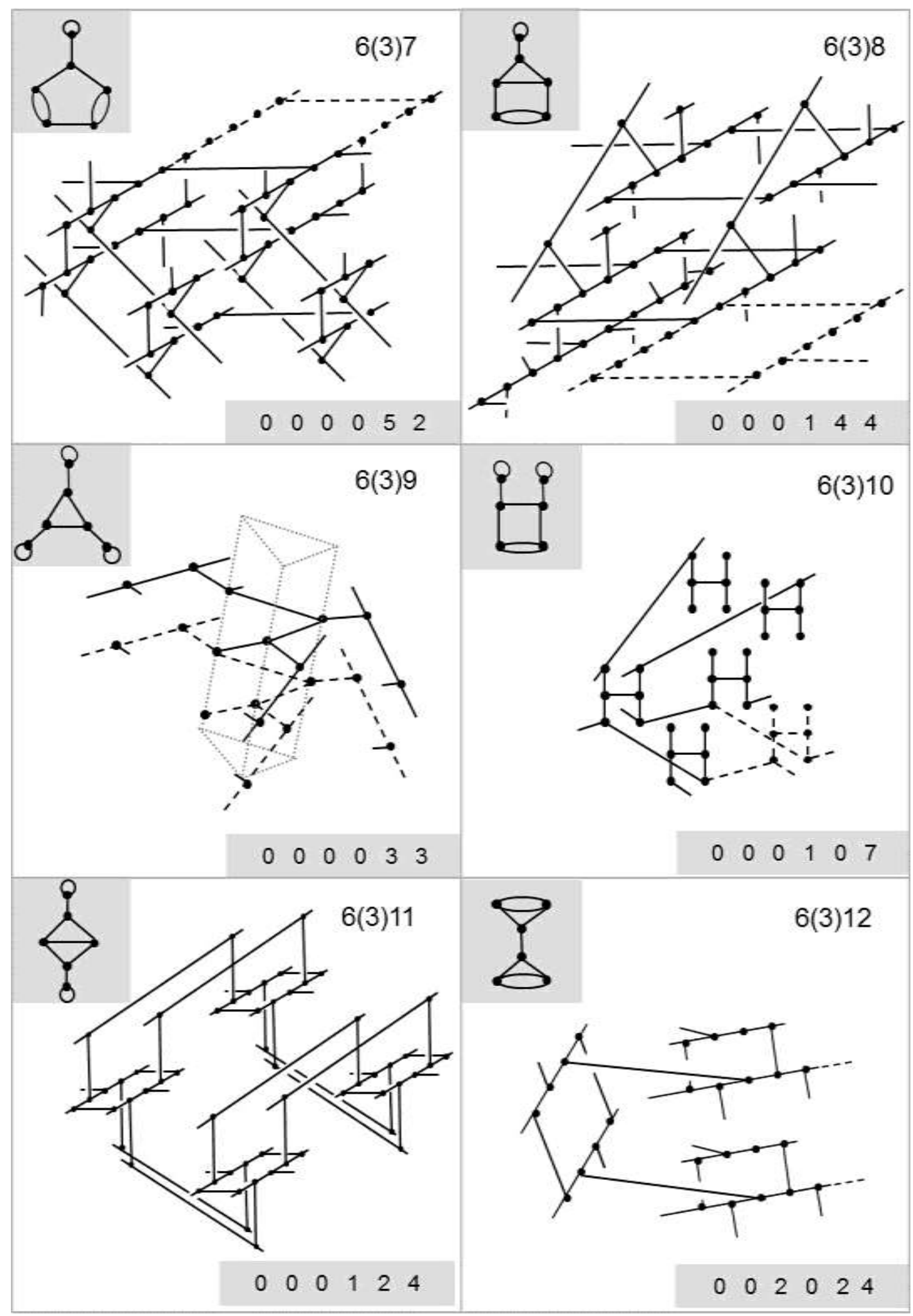




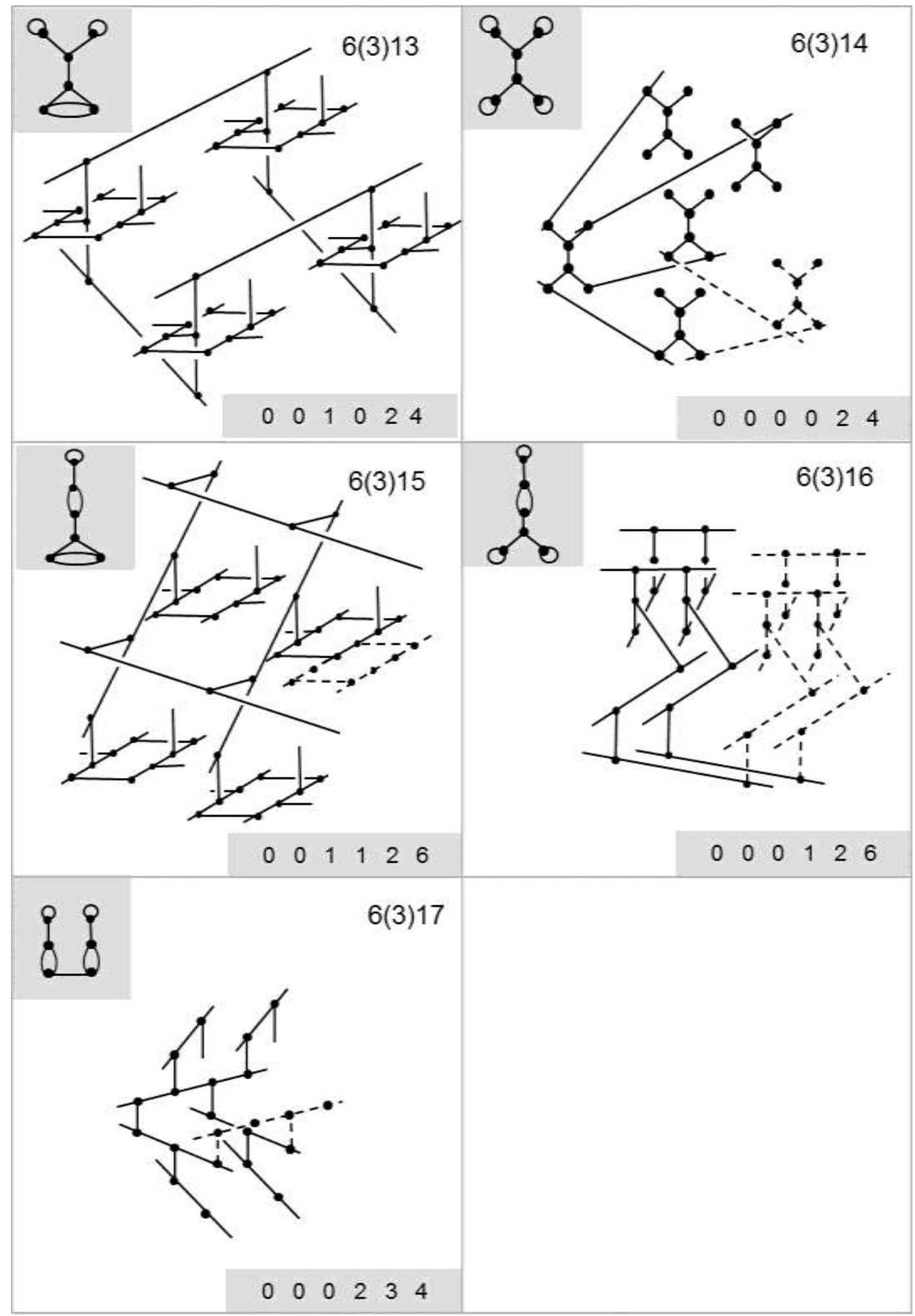




\section{Literatur}

[Baburin, 2008]

I. A. Baburin

Hydrogen-bonded frameworks in molecular metal-organic crystals: the network approach

Z. Kristallogr. 223, 371-381 (2008).

[Bannwarth, Kremer, Schulz, 2010]

H. Bannwarth, B. P. Kremer, A. Schulz

Basiswissen Physik, Chemie und Biochemie

Springer (2010).

[Batten, 2001]

S. Batten

Topology of Interpenetration

CrystEngComm. 3, 67-73 (2001).

[Behrends, Gritzmann, Ziegler, 2008]

E. Behrends, P. Gritzmann, G. M. Ziegler

$\pi$ \& Co. - Kaleidoskop der Mathematik

Springer (2008).

[Beukemann, 1989]

A. Beukemann

Beiträge zur Erzeugung und Klassifikation von Netzen

Diplomarbeit, Institut für Kristallographie der Universität Karlsruhe (1989).

[Beukemann, Klee, 1988]

A. Beukemann, W. E. Klee

The 3-periodic nets with quotient graph $\mathrm{K}_{3,3}$

Z. Kristallogr. 185, 285 (1988).

[Beukemann, Klee, 1992]

A. Beukemann, W. E. Klee

Minimal nets

Z. Kristallogr. 201, 37-51 (1992).

[Beukemann, Klee, 1994]

A. Beukemann, W. E. Klee

Cycle classes as topological invariants of crystal structures

Z. Kristallogr. 209, 714-719 (1994).

[Blatov, 2007]

V. A. Blatov

Topological relations between three-dimensional periodic nets. I. Uninodal nets Acta Cryst. A63, 329-343 (2007). 
[Blatov, Proserpio, 2011]

V. A. Blatov, D. M. Proserpio

Periodic-Graph Approaches in Crystal Structure Prediction

Wiley-VCH Verlag GmbH \& Co. KGaA (2011).

[Bonneau, Delgado-Friedrichs, O'Keeffe, Yaghi, 2004]

C. Bonneau, O. Delgado-Friedrichs, M. O’Keeffe, O. M. Yaghi

Three-periodic nets and tilings: minimal nets

Acta Cryst. A60, 517-520 (2004).

[Brown, Bülow, Neubüser, Wondratscheck, Zassenhaus, 1978]

H. Brown, R. Bülow, J. Neubüser, H. Wondratscheck, H. Zassenhaus

Crystallographic groups of four-dimensional space

John Wiley \& Sons (1978).

[Chung, Hahn, Klee, 1984]

S. J. Chung, T. Hahn, W. E. Klee

Nomenclature and Generation of 3-Periodic Nets: The Vector Method

Acta Cryst. A40, 42-50 (1984).

[Delgado-Friedrichs, O’Keeffe, Yaghi 2002]

O. Delgado-Friedrichs, M. O'Keeffe, O. M. Yaghi

The $\mathrm{CdSO}_{4}$, rutile, cooperate and quartz dual nets: interpenetration and catenation

Solid State Sci. 5, 73-78 (2003).

[Delgado-Friedrichs, O'Keeffe, 2003]

O. Delgado-Friedrichs, M. O'Keeffe

Identification of and symmetry computation for crystal nets

Acta Cryst. A59, 351-360 (2003).

[Delgado-Friedrichs, O'Keeffe, 2005]

O. Delgado-Friedrichs, M. O'Keeffe

Crystal nets as graphs: Terminology and definitions

Journal of Solid State Chem. 178, 2480-2485 (2005).

[Delgado-Friedrichs, et al., 2005]

O. Delgado-Friedrichs, M. D. Foster, M. O’Keeffe, D. M. Proserpio,

M. M.J. Treacy, O. M. Yaghi

What do we know about three-periodic nets?

Journal of Solid State Chem. 178, 2533-2554 (2005).

[Delgado-Friedrichs, O’Keeffe, Yaghi, 2006]

O. Delgado-Friedrichs, M. O'Keeffe, O. M. Yaghi

Three-periodic nets and tilings: edge-transitive binodal structures

Acta Cryst. A62, 350-355 (2006). 
[Delgado-Friedrichs, O’Keeffe, Yaghi, 2007]

O. Delgado-Friedrichs, M. O'Keeffe, O. M. Yaghi

Taxonomy of periodic nets and the design of materials

Phys. Chem. Chem. Phys. 9, 1035-1043 (2007).

[Elisbihani, 2002]

K. Elisbihani

Hochauflösende $\gamma$ - Diffraktometrie zur Untersuchung der Ferroelektrischen

Lock-in Phasenumwandlung in $\mathrm{Rb}_{2} \mathrm{ZnCl}_{4}$

Dissertation, Mathematisch-Naturwissenschaftliche Fakultäten

Georg-August-Universität Göttingen (2002).

[Eon, 1999]

J.-G. Eon

Archetypes and other embeddings of periodic nets generated by orthogonal projection Journal of Solid State Chem. 147, 429-437 (1999).

[Eon, 2004]

J.-G. Eon

Topological density of nets: a direct calculation

Acta Cryst. A60, 7-18 (2004).

[Eon, 2007]

J.-G. Eon

Infinite geodesic paths and fibers, new topological invariants in periodic graphs

Acta Cryst. A63, 53-65 (2007).

[Eon, 2011]

J.-G. Eon

Euclidian embeddings of periodic nets: definition of a topologically induced complete set of geometric descriptors for crystal structures

Acta Cryst. A67, 68-86 (2011).

[Eon, Proserpio, Blatov, 2012]

J.-G. Eon, D. M. Proserpio, V. A. Blatov

Totally unimodular nets

Acta Cryst. A68, 286-294 (2012).

[Fischer, 1973]

W. Fischer

Existenzbedingungen homogener Kugelpackungen zu kubischen Gitterkomplexen mit weniger als drei Freiheitsgraden

Z. Kristallogr. 188, 129-146 (1973). 
[Fischer, 1991]

W. Fischer

Tetragonal sphere packings

Z. Kristallogr. 194, 67-85 (1991).

[Graefe, 1989]

R. Graefe

Zur Geschichte des Konstruierens

Fourier (1989).

[Harary, 1969]

F. Harary

GraphTheory

Eddison-Wesley Publishing Company (1969).

[Kickelbick, 2008]

G. Kickelbick

Chemie für Ingenieure

Pearson Deutschland (2008).

[Kleber, Bautsch, Bohm, Klimm, 2010]

W. Kleber, H.-J. Bautsch, J. Bohm, D. Klimm

Einführung in die Kristallographie

Oldenbourg Wissenschaftsverlag (2010).

[Klein, 2012]

H.-J. Klein

The use of periodic graphs for representing and analysing crystal structures

Z. Kristallogr. 227, 612-618 (2012).

[Klockmann, 1980]

F. Klockmann (erweitert von P. Ramdohr, H. Strunz)

Lehrbuch der Mineralogie

Ferdinand Enke Verlag (1980).

[Krehl, 1969]

H. Krehl (Red., verantwortlich), L. Hornung, U. Sickel

Bauten der Olympischen Spiele 1972 München

Architekturwettbewerbe (1969).

[Lüscher, 2004]

R. Lüscher

Crystal-quasicrystal interfaces: Al on Al-Pd-Mn

Dissertation, Swiss Federal Institute of Technology Zurich

ETH Zürich (2004). 
[Marks, 1960]

R. W. Marks

The Dymaxion World of Buckminster Fuller

Reinhold Publishing Corporation (1960).

[Meisterernst, 2006]

G. Meisterernst

Experimente zur Wachstumskinetik dekagonaler Quasikristalle

Dissertation, Fakultät für Geowissenschaften

Ludwig-Maximilians Universität München (2006).

[O’Keeffe, 1991]

M. O`Keeffe

N-Dimensional Diamond, Sodalite and Rare Sphere Packings

Acta Cryst. A47, 748-753 (1991).

[Penrose, 1979]

R. Penrose

Pentaplexity - a class of non-periodic tilings of the plane

Mathematical Intelligencer 2, 32-37 (1979).

[Prins, 1957]

G. Prins

The automorphism group of a tree

Dissertation, University of Michigan (1957).

[Radke, 1985]

W. Radke

Erzeugung von 3-fach periodischen Graphen als Modelle für Kristallstrukturen

Diplomarbeit, Institut für Kristallographie der Universität Karlsruhe (1985).

[Shechtman, 2013]

D. Shechtman

Quasi periodic crystals - the long road from discovery to acceptance

Ramban Maimonides medical journey 4(1) (2013)

Z. Kristallogr. 219, 391-446 (2004).

[Steurer, 2004]

W. Steurer

Twenty years of structure research on quasicrystals. Part I. Pentagonal, octagonal, decagonal and dodecagonal quasicrystals

Z. Kristallogr. 219, 391-446 (2004). 
[Sunada, 2013]

T. Sunada

Topological crystallography - with a view towards discrete geometric analysis

Springer (2013).

[Thimm, 1991]

G. Thimm

Isomorphie von Netzen

Diplomarbeit, Institut für Kristallographie der Universität Karlsruhe (1991).

[Thimm, 2008]

G. Thimm

A Graph Theoretical Approach to the Analysis, Comparison, and Enumeration of Crystal Structures,

Dissertation im Fachbereich Geowissenschaft

Johann Wolfgang Goethe Universität, Frankfurt am Main (2008).

[Weddigen, Jüngst, 1986]

Ch. Weddigen, W. Jüngst

Elektronik, Einführung für Naturwissenschaftler und Ingenieure

Springer (1986).

[Wells, 1977]

A. F. Wells

Three-Dimensional Nets and Polyhedra

John Wiley \& Sons (1977).

[De Wolff, 1984]

P. M. de Wolff

Dualistic interpretation of the symmetry of incommensurate structures

Acta Cryst. A40, 34-42 (1984). 


\section{Abbildungen}

Abb. 2.7

[Beukemann, 1988, 1990, Folien für Vorträge]

Ausschnitte aus den Folien für die folgenden beiden Vorträge:

- 8.6.89 Universität Karlsruhe, Institut für Kristallographie

- 26.6.90 TU Berlin, Institut für Kristallographie und Mineralogie

Tab. 2.1

[Bonneau, Delgado-Friedrichs, O'Keeffe, Yaghi, 2004]

Ausschnitt aus Table 1; Acta Cryst. A60, S. 518 (2004).

Tab. 2.2, 5.-7. Spalte

[Bonneau, Delgado-Friedrichs, O'Keeffe, Yaghi, 2004]

8 kopierte Ausschnitte aus Figure 2; Acta Cryst. A60, S. 518 (2004).

Abb. 9.8, Foto

Dreidimensionales Modell: A. Beukemann

Baukasten: Creator 1, Zometool, 2010, www.zometool.com

Fotographie und Fotobearbeitung: J. Saeftel, 08/2014

(Alle anderen Abbildungen wurden von A. Beukemann erstellt) 


\section{Lebenslauf}

Alexander Beukemann

Geburtsdatum:

14.08.1957 in Stuttgart

Familienstand: $\quad$ verheiratet, zwei Kinder (18 und 20 Jahre)

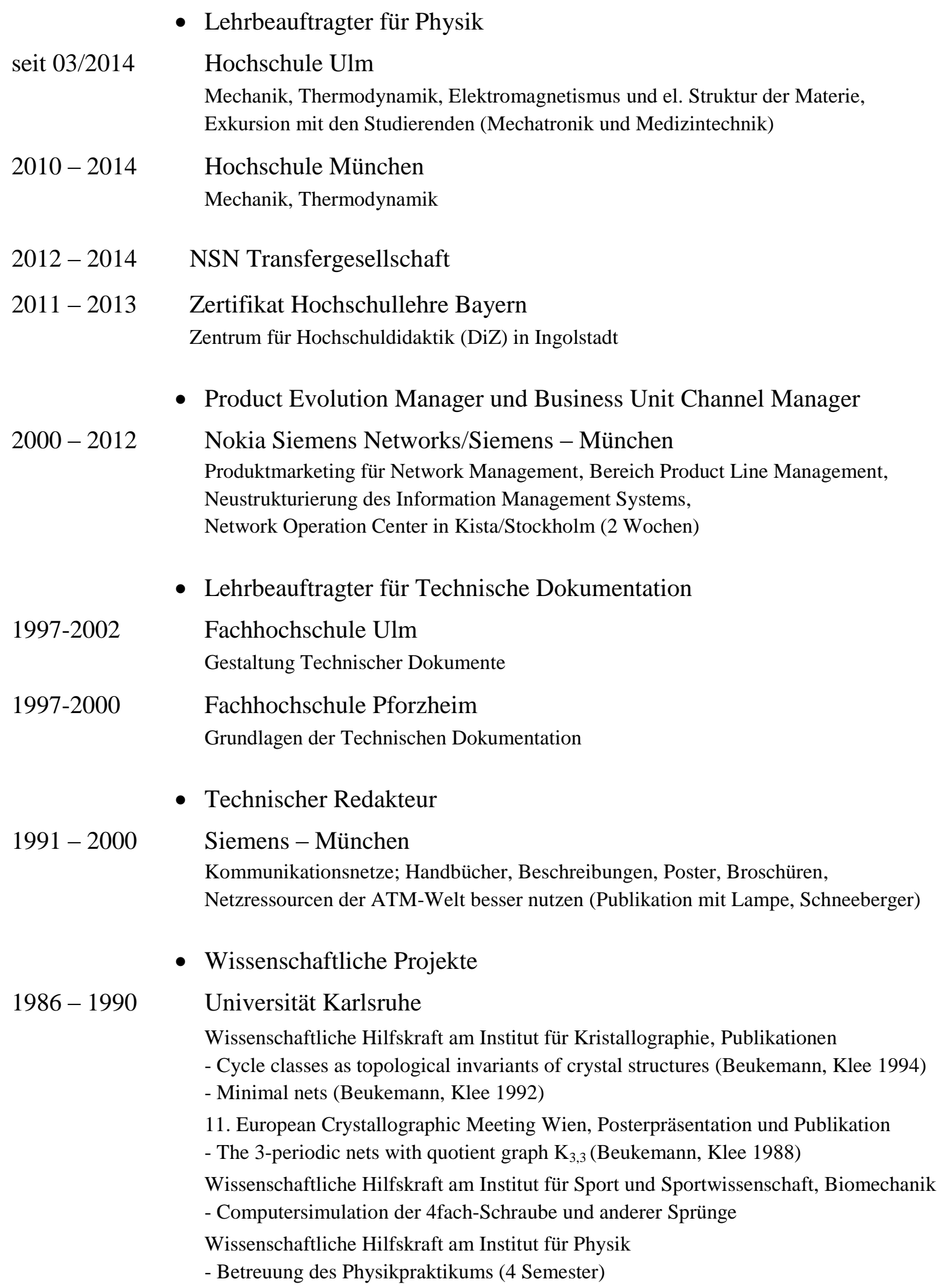

- Lehrbeauftragter für Physik

seit 03/2014 Hochschule Ulm

Mechanik, Thermodynamik, Elektromagnetismus und el. Struktur der Materie, Exkursion mit den Studierenden (Mechatronik und Medizintechnik)

2010 - $2014 \quad$ Hochschule München

Mechanik, Thermodynamik

$2012-2014 \quad$ NSN Transfergesellschaft

2011 - 2013 Zertifikat Hochschullehre Bayern

Zentrum für Hochschuldidaktik (DiZ) in Ingolstadt

- Product Evolution Manager und Business Unit Channel Manager

2000 - $2012 \quad$ Nokia Siemens Networks/Siemens - München

Produktmarketing für Network Management, Bereich Product Line Management, Neustrukturierung des Information Management Systems,

Network Operation Center in Kista/Stockholm (2 Wochen)

- Lehrbeauftragter für Technische Dokumentation

1997-2002 Fachhochschule Ulm

Gestaltung Technischer Dokumente

1997-2000 Fachhochschule Pforzheim

Grundlagen der Technischen Dokumentation

- Technischer Redakteur

$1991-2000 \quad$ Siemens - München

Kommunikationsnetze; Handbücher, Beschreibungen, Poster, Broschüren,

Netzressourcen der ATM-Welt besser nutzen (Publikation mit Lampe, Schneeberger)

- Wissenschaftliche Projekte

1986 - $1990 \quad$ Universität Karlsruhe

Wissenschaftliche Hilfskraft am Institut für Kristallographie, Publikationen

- Cycle classes as topological invariants of crystal structures (Beukemann, Klee 1994)

- Minimal nets (Beukemann, Klee 1992)

11. European Crystallographic Meeting Wien, Posterpräsentation und Publikation

- The 3-periodic nets with quotient graph $\mathrm{K}_{3,3}$ (Beukemann, Klee 1988)

Wissenschaftliche Hilfskraft am Institut für Sport und Sportwissenschaft, Biomechanik

- Computersimulation der 4fach-Schraube und anderer Sprünge

Wissenschaftliche Hilfskraft am Institut für Physik

- Betreuung des Physikpraktikums (4 Semester) 
- Physik-Studium

1986 - $1990 \quad$ Universität Karlsruhe

Abschluss: Diplom

- Studium für das Lehramt an Gymnasien - Sport und Physik

1978 - $1986 \quad$ Universität Karlsruhe

Abschluss 1. Staatsexamen

Tutor im Arbeitskreis Sport, Studentenwerk Karlsruhe

$1980-1981 \quad$ I.N.S.A. Lyon

Auslandsstipendium am Institut National des Sciences Appliquées de Lyon

Génie Physique Matériaux

$1977-1978 \quad$ Bundeswehr

1977

Abitur am Humboldt-Gymnasium Ulm

Interessen:

Sport

Übungsleitertätigkeiten im Ski-Club FFB:

Skilanglauf (ab 2008)

Skikurse für Kinder (1998-2009)

Skigymnastik für Erwachsene (1997-2003)

Musik

Gitarre, Schlagzeug 